\title{
Gas sensors based on mass-sensitive transducers. Part 2: Improving the sensors towards practical application
}

\author{
Alexandru Oprea ${ }^{1,2} \cdot$ Udo Weimar $^{1,2}$ \\ Received: 20 August 2018 / Revised: 24 February 2020 / Accepted: 27 March 2020 / Published online: 31 July 2020 \\ (C) The Author(s) 2020
}

\begin{abstract}
Within the framework outlined in the first part of the review, the second part addresses attempts to increase receptor material performance through the use of sensor systems and chemometric methods, in conjunction with receptor preparation methods and sensor-specific tasks. Conclusions are then drawn, and development perspectives for gravimetric sensors are discussed.
\end{abstract}

Keywords Gravimetric gas sensors · Functionalized sensing materials · Deposition methods · Targeted applications

\section{Short introduction to the second part of the review}

As discussed in the introduction to the first part of the review [1], the development of gas sensors (GS) was driven by the increasing need for the detection of environmental, industrial and domestic chemical hazards. As a result of sustained investigations performed in the 1980s and 1990s, a greater understanding of gas sensing mechanisms based on mass-sensitive transducers (MST) was achieved with regard to both reception and transduction processes. However, for practical purposes, the application of this knowledge did not translate to the production of commercial devices. The main reason for this failure was the poor performance of the receptor materials, especially their low specificity. Section 2 of the second part of the review tackles this issue and presents the modalities investigated in studies aimed at increasing receptor specificity. Two main approaches are considered: increasing the specificity of the receptor-analyte interaction, and employing gas sensor systems (GSS) containing several individual devices with limited specificity in combination with chemometric methods in

Alexandru Oprea

alexandru.oprea@ipc.uni-tuebingen.de

1 Institute of Physical and Theoretical Chemistry, Eberhard Karls University, Tübingen, Germany

2 Center for Light-Matter Interaction, Sensors \& Analytics, Eberhard Karls University, Auf der Morgenstelle 15,

72076 Tübingen, Germany order to increase the overall gravimetric selectivity. The main task of either GS or GSS is the same, namely, to provide a specific and proportional response to the concentration of the analytes in the gaseous sample, ultimately enabling the determination of the sample composition or sample classification. In the third section, methods currently utilized for receptor material preparation/deposition are discussed, while the fourth section reviews attempts to improve gas detection specificity, beyond the receptor, through suitable processing of sensor arrays (SA) and GSS data. The fifth section is dedicated to the practical applications of GGSs. The first part discusses the targeted analytes, their main properties, hazards involved and legally allowed concentrations, while the second part presents a survey of applications. The conclusion focuses on the degree to which the advancements in the field of GGSs meet the expectations they have raised.

\section{Increasing the specificity of the receptors}

The interactions addressed in the section "Specific interactions and their role in receptor sensitivity and selectivity" of the first part of the review confer certain selectivity to the sensing process. However, when examining the tables with the values of solvation parameters for the specific versus nonspecific contributions (given by the product $l \cdot \log L^{16}$ ), it becomes obvious that in most cases, the specificity is rather low and is usually restricted to classes of analytes and not to a certain one. Because overcoming these limitations in the context of thermodynamics is very challenging, attempts have been made beyond thermodynamic-controlled specificity, 
searching for receptor materials whose selectivity derives from special features of the analyte-receptor interaction. In this respect, Hierlemann et al. devised combined optical and gravimetric experiments ${ }^{1}$ on the same receptor samples to directly probe the strength and specificity of the analytereceptor interaction [2]. Strong correlations between changes in the infrared spectra and gravimetric sensor signals on the one hand, and the analyte type and concentration on the other hand, occurred when suitable analyte-receptor ${ }^{2}$ combinations were chosen. On the contrary, when analyte detection was performed with common polymers, poly(ether urethane) and poly(isobutene), the receptor response was weak and nonspecific, as expected. It is worth noting here that the laws of thermodynamics always hold, and the dispersion interaction will contribute significantly to the sensor output, regardless of how well tailored the receptor is. Therefore, in the analyte adsorption isotherm, the dispersion interaction is seen as a nonspecific contribution following Henry's law superposed to the specific contribution (a Langmuir dependency on analyte concentration for low exposure levels) [2,3], as depicted in Fig. 1. Moreover, the stronger the analyte-receptor interaction, the less reversible and slower the sensor.

\section{Molecular recognition}

The specificity ensured by the complementarity of the solvation parameters, as they appear in the "linear solvation energy relationship" $[4,5]$ (see the first part of the review, section "Bulk receptors in the frame of the linear solvation relationship"), can be improved by mimicking the biological systems (like DNA chains) [6]. Several examples of biological binding of gases, among which that of $\mathrm{O}_{2} / \mathrm{CO}$ by heme molecules is the most well known, are given by Rudkevich in his review "Emerging Supramolecular Chemistry of Gases" [7]. The advances in the chemistry of the biological world led to the "molecular recognition" and "supramolecular chemistry" concepts and models. In its common meaning, molecular recognition describes the specific interaction between two molecules (one larger-the molecular receptor, and one smaller-the substrate ${ }^{3}$ ) through complementary noncovalent bonding (also included here are the specific interactions addressed in the first part of the review such as metal coordination or hydrophobic interaction). According to Lehn, "mere binding is not recognition", so that molecular recognition is "a process involving both binding and selection of

\footnotetext{
${ }^{1}$ Experiments making use of Fourier transform infrared external reflectance spectra and functionalized surface acoustic wave devices.

${ }^{2}$ The authors refer to cyclodextrins for chiral recognition, nickel camphorates for Lewis bases such as pyridine or organophosphonates, and phthalocyanines for aromatic compounds for which specific chemical interactions such as metal coordination, "cage" compound inclusion, or $\pi$-stacking were expected (chiral recognition in general will be addressed in a later subsection of this review).

${ }^{3}$ The term "substrate" is used for enzymatic reactions. For sensing processes, the appropriate concept is "analyte".
}

substrate(s) by a given receptor molecule" [8]. In Lehn's view, molecular recognition involves a "double complementarity principle extending over energetical (electronic) as well as geometrical features, the celebrated 'lock and key', steric fit concept enunciated by Emil Fischer" in 1894 [9]. The result of molecular recognition is a supramolecular system which stores a certain amount of information (architecture of ligands, binding sites, etc.) [8]. Similarly, Cram regards molecular recognition as a guest-host interaction resulting in a guest-host complex [10]. The most relevant complementary characteristics stem from size, shape, charge, dipolar momentum and acidity-basicity of the hydrogen bond. Starting from the old "lock-and-key" picture by Fischer [9], Rebek synthesized molecular clefts for selected analytes as model receptors with high selectivity [11]. Even the formation of the U-shaped model molecule from the building blocks ( $2 x$ Kemp's triacid and naphthalene-2,7-diamine in the simplest example) was due to chemical affinity, complementarity and steric barriers. The free carboxylic groups of the cleft prepared in this way can specifically "catch" two isopropanol molecules, forming hydrogen bonds with the hydroxy groups of the alcohol. Here the appropriate size of isopropanol molecules plays an important role. However, the complementarity required for the formation of the guest-host complexes with specific receptors, leading to molecular recognition, is generally less available for the gaseous analytes because of limited dimensions, shapes and polarizability [7]. The topic of "Molecular recognition and supramolecular chemistry in the gas phase" is specifically referred to by Schalley [12].

Many natural, biological or common synthetic sensing materials employed for gas-phase detection possess certain intrinsic complementarity with respect to some target gases, so that in this case the selectivity is ensured by appropriate selection of the receptor-analyte pair through a trial procedure. In order to achieve the sensing performance required by practical applications, it is necessary to use different approaches, based on rational design and preparation (often through synthesis) of tailored receptor material for the given analytes. The natural/biological and synthetic receptors are presented in the respective sections below. The examples selected from the literature to better illustrate the receptor-analyte interaction are only roughly presented in those sections (they will mainly address the receptor material features and target analyte nature or composition). The bestperforming sensors are discussed in more detail in the section dedicated to applications, pointing to the sensing parameters. Some of the experimental approaches fit more than one type of molecular recognition; in the following, a classification choice has been made, or they were considered twice.

\section{Natural and biological receptors}

The living world abounds in good receptors for gases and vapors, integrated in sophisticated olfactory systems which 
are able to recognize the chemical nature of the gaseous environment. The path from reception to olfactive perception is complex and includes chemical, biochemical, physiological and, at least for humans, psychologic stages [13-16]. In the case of mammals (including humans) the olfactory process starts in the nose and ends in the brain [17]. First, the odorant is reversibly attached to the small soluble proteins - the odorant-binding proteins (OBP) [18-27] - secreted in the nasal mucus ${ }^{4}$. Thus, the OBPs are accessible to a large number of olfactory receptors (OR) in the nasal epithelium. The actual reception takes place when the chemical components of the odors are released from the OBPs to bind on the heptahelical protein coupled with the G-protein in the cilia of the sensitive neurons in the olfactory epithelium [31-33]. The specificity of the G-protein-coupled receptors (GPCR), which is rather limited, is encoded on the specific multigene family [34, 35]. Discrimination of the odors at the physiological level makes use of a combinatory approach [36-38]. Accordingly, one gaseous analyte is recognized by several GPCRs, but it is specifically detected only by one combination of them. Using olfactory organs based on dozens to thousands of olfactory codes (genes), animals can discern two to three orders of magnitude larger numbers of smells ${ }^{5}$. After probing their ability to specifically bind gases/vapors [28, 39-41], different elements of the biological odorant recognition systems, supported by adequate mathematical algorithms, have been exploited towards gas sensors, as sketched in Fig. 2 [42-46]. The simpler receptor materials (peptides, proteins, and even ORs) are more stable than the complex ones (olfactory neurons, nasal epithelium, cell cultures of olfactory tissues) and are therefore more often used in the applications. Many practical approaches employ impedimetric [39, 47, 48], fieldeffect $[49,50]$, and electrical transducers, but there are also several examples of GS based on MSTs.

Sankaran et al. used OBP LUSH peptides from Drosophila, self-assembled on a quartz (Q) thickness shear mode resonator (TSMR), to detect heavy alcohols (3-methyl-1-butanol and 1-hexanol) associated with Salmonella contamination in packaged beef. In principle, sensitivity of $\sim 0.1 \mathrm{~Hz} / \mathrm{ppmv}$ with a lower limit of detection ${ }^{6}$ (LDL) below 5 ppmv should be enough to accomplish the task. However, no experiments were performed to identify Salmonella in real samples. The separation of the two alcohols addressed above using principal component analysis (PCA, see section "Evaluating the performance of the gas sensors and sensor

\footnotetext{
${ }^{4}$ In the case of insects and arthropods, the OBPs are secreted in the sensillum lymph, having accessibility to a restricted number of odorant receptors inside a sensillum only, but having greater diversity [25, 28-30]

5 Humans possess $\sim 350$ "olfactory" genes but are able to distinguish more than 100,000 smells [37].

${ }^{6} \mathrm{LDL}$ and all other parameter describing the performance of the sensors will be addressed at the beginning of the section "Targeted analytes" below)
}

arrays" below) was good, but humidity was not considered at all.

GGS sensor arrays consisting of gold nanoparticles modified with different peptides were employed by Compagnone et al. to detect food aromas (cis-3-hexenol, terpinen-4-ol, ethyl acetate and isopentyl acetate at $0.1 \%$ volume in ethanol, acetonitrile, acetone and hexane as solvents) [51]. Their PCA discrimination from the headspace atmosphere, created through $\mathrm{N}_{2}$ bubbling of solutions, was rather good. Water was excluded from the solvent list, even though the sensor array has been calibrated against humidity, because $\mathrm{H}_{2} \mathrm{O}$ slowed the reception mechanism.

Sensitive detection of VOCs (octenol, carvone) with wildtype (wt) double-mutant (dm) bovine (b) and porcine (p) OBPs, respectively, was reported by Di Pietrantonio et al. [52]. The authors used two-ports $392 \mathrm{MHz}$ surface acoustic wave (SAW) transducers drop-coated with OBP solutions in an array configuration (three sensing devices and an uncoated reference device). They obtained linear calibration curves (see Fig. 3) having maximal sensitivity of $25.9 \mathrm{~Hz} / \mathrm{ppmv}$ and LDL of 0.39 ppmv for octenol when measured with wtpOBP-based sensors. The influence of temperature changes and humidity background was compensated by the differential readout of the sensors, each with respect to the reference SAW device. Zhao et al. proposed a thin film bulk ultra-acoustic $(\sim 1.5 \mathrm{GHz})$ resonator (FBAR) as transducer for a protein (AaegOBP22)functionalized odorant biosensor. Saturated vapor (200 ppmv $^{7}$ ) of the $N, N$-diethyl-3-methylbenzamide (DEET) target analyte was clearly detected, but with rather large noise [54].

Relatively recent approaches have evaluated the potential specificity and binding strength of peptides and proteins towards given gaseous analytes through molecular simulation methods [55], virtual screening [56, 57] and in silico "experiments" [58]. Synthetic poly(peptides) with the sequence "RVNEWVIC", found to be selective for acetic acid by Wu et al. [55], were practically tested by Panigrahi et al. with GGSs based on TSMRs [59]. Sensitivity towards acetic acid of about $0.1 \mathrm{~Hz} / \mathrm{ppmv}$ and LDL of 2 ppmv satisfied application requirements. The humidity influence was assumed to be minimal based on chemo-physical justifications, but no dedicated measurements were carried out. The achievements based on virtual screening and in silico approaches will be addressed below in the section "Gravimetric sensor systems", as they better fit this topic.

\section{Synthetic/tailored receptors}

Because the long-term stability of the biological receptors is rather poor, many researchers have replaced them with

\footnotetext{
${ }^{7}$ The given value of the concentration was inferred by the authors of the present review from the saturation vapor pressure provided by [53].
} 
Fig. 1 The adsorption isotherms at low analyte concentrations. Molecular recognition results in superposed Henry and Langmuir isotherms. Reproduced with the kind permission of ACS

Publications from reference [2]

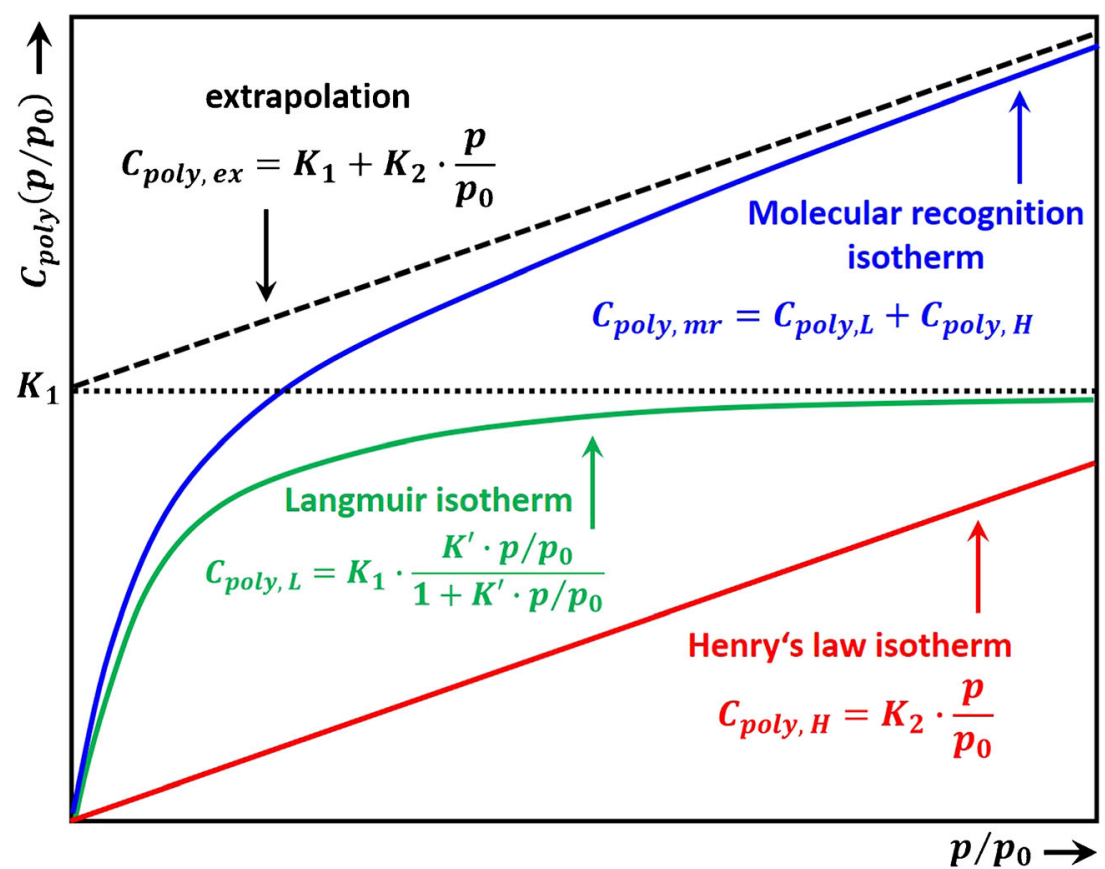

synthetic materials having engineered sensing properties. The most successful classes are discussed below.

Molecularly imprinted polymers Molecularly imprinted polymers (MIP) have been devised as synthetic hosts able to provide increased affinity and specificity towards target/guest molecules. They mimic the biological systems from which they were inspired, with certain advantages in terms of chemical stability, long-term preservation of the strength and specificity of the binding sites, mechanical reliability, pressure and temperature durability, ease of preparation and low cost $[60,61]$. However, they have some drawbacks (are large, rigid and insoluble) with respect to the biological receptors, which are usually smaller, flexible and mainly soluble [60]. The first approach to MIPs dates back to 1931, when Polyakov's group succeeded in synthesizing silica gel with unusual adsorption
Fig. 2 Sensors based on biological receptor materials. Advantages in green and drawbacks in red. Reproduced with the kind permission of Elsevier B.V from reference [42]

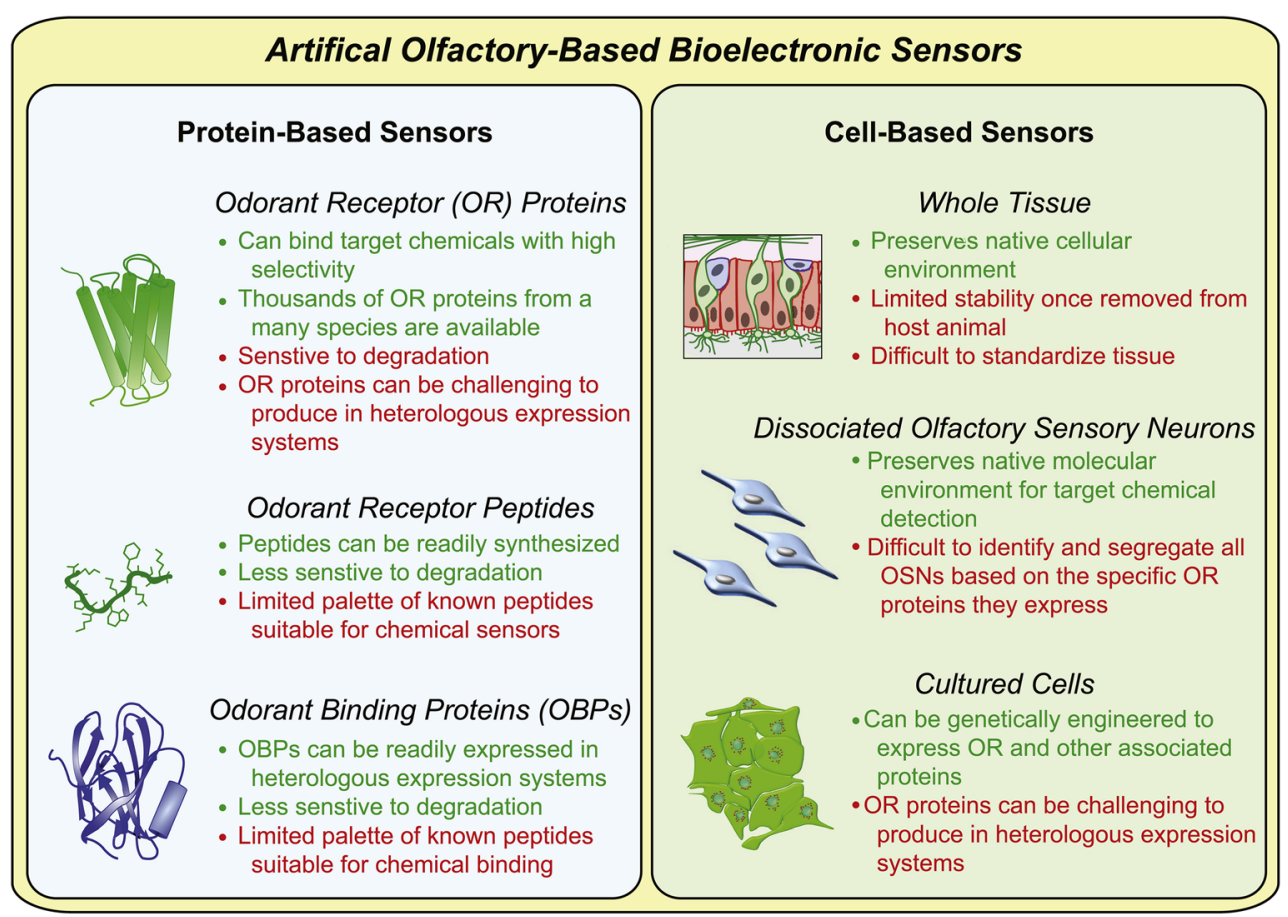


Fig. 3 Calibration curves of three sensors coated with different OBPs (see the text for assignation) towards octenol. Reproduced with the kind permission of Elsevier B.V. from reference [52]

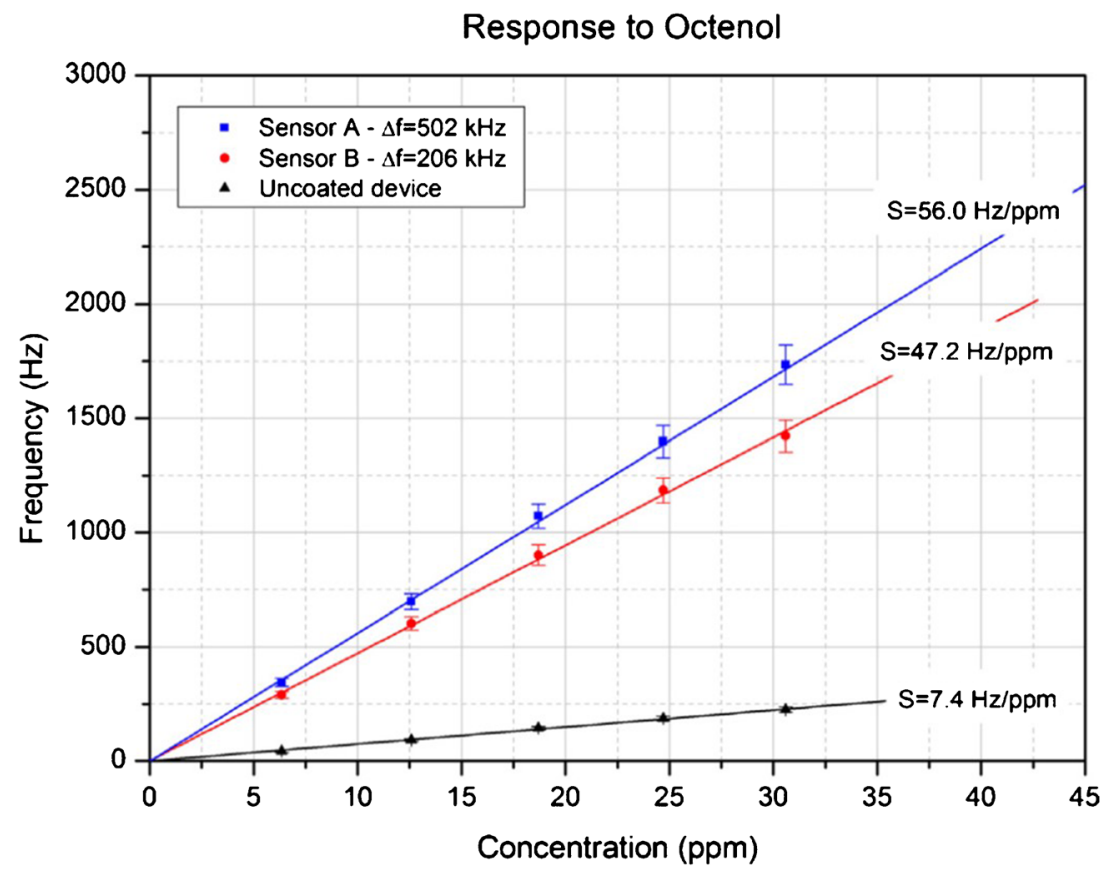

properties towards benzene, toluene and xylene [62] (this work is briefly described in the ample review by Whitcombe et al. [63]). Since then, a huge number of investigations have been performed and their achievements reported in the literature. Several relevant review are available [12, 60, 61, 63-79]. Very roughly, the main idea behind MIP preparation is to add a template (target/guest molecule or a suitable substitute) and functionalized monomers to the MIP precursors to enable polymerization, and to remove the template after polymer synthesis. The resulting host material — the MIP — would have numerous suitably shaped cavities possessing the complementarity required in molecular recognition. There are a few dedicated routes for the manufacture of MIPs, which are sketched in Fig. 4.

The covalent route [80] relies on reversible covalent binding of the template with the reacting monomers. The stoichiometry is ensured by the covalent type of the bonds. After copolymerization with the cross-linker, the template is disrupted by chemical cleavage (acid hydrolysis for instance). Unfortunately, the number and variety of the MIPs obtained in this way is rather limited by the scarce choice of suitable templates and compatible monomers. The non-covalent route
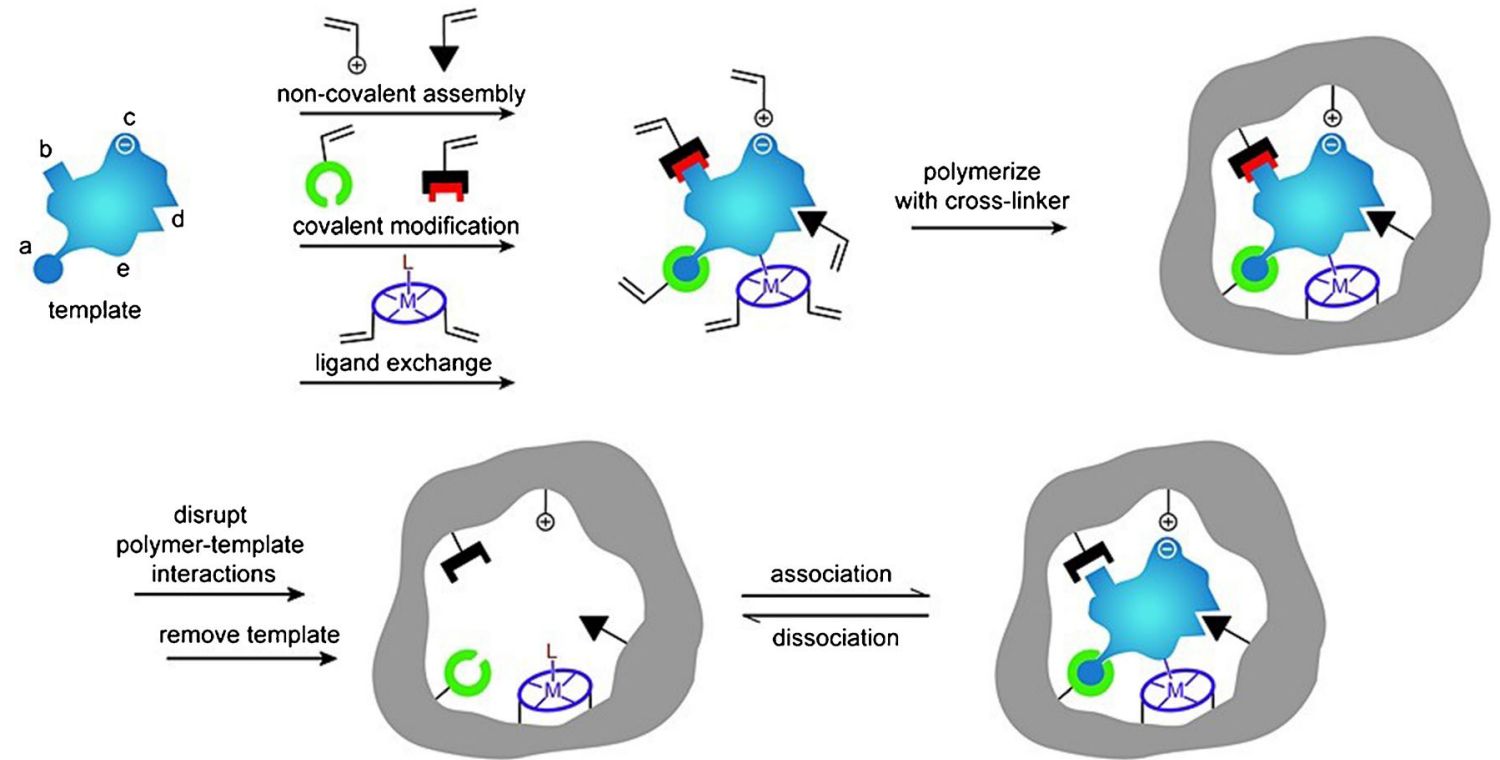

Fig. 4 MIP preparation routes. The routes are described in the text. Reproduced with the kind permission of John Wiley and Sons from reference [63] 
[81] uses a liquid phase process involving the template, functional monomers, cross-linker, initiator and a solvent. The template, which must be stable under preparation conditions, spontaneously binds the functionalized monomers (selfassembly process) by specific weak interactions. With the aid of the initiator, which provides free radicals for the early stages of the chain polymerization reaction when thermally or optically triggered, the template-functional complex copolymerizes with the cross-linker towards a template containing MIP. In a final preparation step, the template is removed by dissolution, and the MIP is able to rebind the template at the recognition sites. Besides the role played in the synthesis, controlling the morphology of the polymer matrix, the crosslinker stabilizes the imprinted binding locations and confers mechanical stability to the MIP, while the solvent determines its porosity [70]. There are a few other ways to realize the imprint: stoichiometric non-covalent approach, semicovalent approach, metal ion-mediated approach [63] and the sol-gel route [82]. With the early polymer imprinting techniques, the choice of the reactants and reaction conditions was mainly a matter of "chemical intuition", and the desired MIP specificity was gathered by long and unsystematic investigations, involving significant experimental efforts. The combinatorial methods [83] have improved the efficiency of the screening in molecular imprinting, making use of automated trial procedures. The rational MIP design, using thermodynamic and physicochemical foundations, constituted a real step forward in the field $[84,85]$. The state of the art is represented by modeling of the template interaction with the possible functionalized monomers in the framework of molecular mechanics/dynamics, empirical/semiclassical quantum mechanics or ab initio quantomechanical formalisms (HartreeFock, Møllere-Plesset, DFT) [86-88]. In spite of the level reached by the theoretical and experimental approaches to MIP preparation, the specific recognition of target molecules is rather limited because of the relatively large amount of cross-linkers typically used in the synthesis routes, which allow for significant contributions from nonspecific binding mechanisms (mainly through dispersion interactions).

$\mathrm{Fu}$ and Finklea generated two types of shape-selective cavities (with hydroquinone and phenol non-covalently bound templates) in a poly(acrylic) or poly(methacrylic) polymer matrix [89]. The polymers were coated on the sensing TSMRs in a differential sensor system through an interfacing poly(isobutylene) film. Good response towards volatile organic compounds (VOC), linear calibration curves, short response $(5 / 12 \mathrm{~s})$ and acceptable recovery (11/90 s) times were achieved. The sensitivity was proportional (but not directly proportional) to the sensing layer thickness. This imprinting procedure conferred additional sensitivity and selectivity and changed the cross-sensitivity ratios among the tested analytes (trichloroethylene, benzene, toluene, heptane and carbon tetrachloride). Unfortunately, the humidity was omitted from the cross- sensitivity test. Starting from similar monomers, methacrylic acid and acrylamide, Bunte et al. obtained MIPs imprinted with 2,4,6-trinitrotoluene (TNT) and 2,4-dinitrotoluene (DNT) with high sensitivity and specificity [90]. An analogous approach was successfully used by Kikuchi et al. for the selective detection of terpenes (limonene, limonene oxide and $\alpha$-pinene) [91]. From 4-vinyl pyridine (4VP), 1,4-divinyl benzene (DVB) and 2,2-azobis(isobutyronitrile) (AIBN) as functional monomer, cross-linker and initiator, Hwang et al. synthesized MIPs imprinted with acetoin and phenol for selective detection of isopropyl methyl ketone (IMK) and toluene, respectively [92].

Cavitand receptor materials Compounds whose molecules contain internal free regions, such as porphyrins [93-95], metal-free ${ }^{8}$ phthalocyanines [96], calixarenes [97-100] and corroles [101], can in principle capture foreign atoms with appropriate size and chemical properties. For example, the cavity diameter of the calix $[n]$ arenes with $n=4,6,8$ influences the sensitivity towards chloroform, reaching the maximum for $n=8$ [102]. Q-TSMR arrays coated with porphyrins have been successfully employed for the quality control of chocolate [103] and identification of microorganisms [104]. The approaches ware based on specific combinations of VOCs present in the vicinity of analyzed systems which could be recognized with multivariate data analysis (MDA). For all data evaluation methods ${ }^{9}$ addressed from here on, please refer to the section "Evaluating the performance of the gas sensors and sensor arrays" below. Another cavitand material type, GUMBOS (acronym for "group of uniform materials based on organic salts)" based on cyclic tetrapyrroles such as phthalocyanines and porphyrins, have been produced and used with quartz-TSMRs (Q-TSMRs) for detection of VOCs (methanol, ethanol, 1-propanol, 2-propanol, 1-butanol, acetone, chloroform, toluene, etc.) [105]. The phthalocyanines (Pc) without central atom(s) are currently not reported as gas-sensitive (by any transduction modality), while several metal Pcs have been successfully used for such purposes. One should note that in the case of metal-Pcs, or more generally, of all functionalized cavitands, the favorable chemical interaction can prevail over the geometric matching, and the ad/absorption does not take place, as expected, at the location with complementary shape, but at the chemically suitable sites. For instance, Harbeck et al. synthesized 2,3,9,10,16,17,23,24-octakis-( 7,11 dioxaheptadecane-9-oxo) phthalocyanine and its $\mathrm{Ni}$ and $\mathrm{Co}$ derivatives and investigated their sensing potential towards several classes of VOCs with multi-reflection attenuated total

\footnotetext{
${ }^{8}$ Here, metal-free does not mean a phthalocyanine without any central atom, but a $\mathrm{H}_{2}$ phthalocyanine.

${ }^{9}$ Data evaluation methods addressed are multivariate data analysis and artificial intelligence procedures.
} 
reflection (ATR) Fourier transform infrared (FTIR) spectroscopy and TSMRs [106]. From the assignation and intensity of the ATR-FTIR bands relevant for the sensing process ${ }^{10}$ and TSMR large responses towards nonpolar VOCs ( $n$-hexane), the authors deduced an analyte binding to the substituent alkoxy groups of the Pc receptor through van der Waals interactions. The polar compounds (methanol, ethanol, acetonitrile, ethyl acetate) were better detected with fluorinated alkyloxysubstituted Pcs [107, 108].

Functionalized receptor materials It is difficult to find materials which have, at the same time, good compatibility with the transducer, increased physical and chemical stability, and good gas-sensing properties. The functionalized receptor materials are a class of materials generally possessing the first two requirements, but which gather the gas sensitivity/ specificity either by attaching chemical active groups, particles or nanostructures, or through intentional modification of the local material structure. One always seeks better molecular complementarity of the receptor to the analyte and, by that, a convenient increase in the receptor performance. The functionalization can be made individually, using the chemical affinity of the added entities to the host material, or by devising classes of materials which provide functionalization sites and high gas sorption capabilities.

\section{Functionalization of different plain host materials.} Historically, the specific functionalization was the first modality employed by the researchers to improve the gas-sensing performance of plain sensing polymers, cavitands and twodimensional (2D) or one-dimensional (1D) materials [109, 110]. A systematic investigation on poly(siloxanes) by Hierlemann et al. proved that a suitable choice of the side groups attached to the polymers increases the partition ratio of the targeted analytes [111]. Many researchers have reported good results in gas/vapor sensing with modified polymers [112-114]. To upgrade the sensing performances of cavitands, they were functionalized with side groups like tetra-tert-butyl [115] and alkoxy [106] for phthalocyanines or dihydroxyphenyl for corroles [101], or combined in complex compounds/mixtures [93, 95, 105, 116]. Even though low-dimensional materials have been regarded as intrinsically more sensitive and selective than bulk materials, many researchers are striving to further improve their sensitive features by attaching different nanoparticles or functional structures. Zong et al. successfully functionalized hollow mesoporous silica spheres with poly(dopamine) to detect

\footnotetext{
${ }^{10}$ The ATR-FTIR spectra were recorded for the synthesized Pcs alone and together with ad/absorbed analyte. The difference between the two aboveaddressed spectra (difference spectra) and the known analyte spectra enabled the identification of the vibrations specific to the absorbent-absorbate complexes.

${ }^{11}$ The results will be addressed in the section "Targeted analytes" below.
}

formaldehyde released from food ${ }^{11}$ [117]. Bonding p-hexafluoroisopropanol aniline to mesoporous $\mathrm{TiO}_{2}-\mathrm{SiO}_{2}, \mathrm{Zhu}$ et al. measured concentrations of nerve agent simulant dimethyl methyl phosphonate (DMMP) down to $100 \mathrm{ppbv}$ [118]. The sensing material was coated on Q-TSMRs. Data regarding the influence of humidity on the sensor response are missing. Ogimoto et al. demonstrated the suitability of mesoporous functionalized $\mathrm{SiO}_{2}$ nanoparticle and films for the detection of low levels of ammonia in human breath [119]. A Cu(II) complex $\left[\mathrm{Cu}(\mathrm{DDS})_{2}(\mathrm{Cl}) 2(\mathrm{MeOH})_{2}\right]^{12}$ ( see Fig. 5) for formaldehyde sensing was designed and synthesized by Wang et al. directly on $\mathrm{Cu}$-coated Q-TSMRs, ensuring intimate coupling between receptor and transducers [120]. According to the authors' DFT calculations, the reported sensitivity (LDL of $50 \mathrm{ppbv}$ ) and selectivity are due to the reversible binding of formaldehyde on the amino groups of the complex compound. The reported low cross-sensitivity to humidity is misleading because the water concentration in the test mixture was only 100ppmv.

Although they are good conductors, low-dimensional carbon materials ${ }^{13}$ [121] like carbon nanotubes (CNT) [122-124], graphene [125-129] and reduced graphene oxide (RGO) $[130,131]$ are often functionalized [132] (see Fig. 6) [133], and used not only for conductometric [134] or fieldeffect [135] gas sensors, but also for gravimetric ones. Asad et al. devised SAW $\mathrm{H}_{2} \mathrm{~S}$ gas sensors based on single-walled CNTs decorated with $\mathrm{Cu}$ [136]. These GGSs have LDL below $1 \mathrm{ppmv}$ at room temperature and a reduced cross-sensitivity to humidity ( 2 ppmv $\mathrm{H}_{2} \mathrm{~S}$ roughly corresponds to $40 \%$ relative humidity $[\mathrm{RH}])^{14}$. Phthalocyanine- and porphinfunctionalized CNTs were used by Ndiaye et al. to detect aromatic VOCs with detection limits below the threshold limit values (TLV)/time-weighted averages (TWA) for these analytes [137]. RGO is the most widely used lowdimensional carbon-based material for gas sensing, mainly functionalized. Yu et al. employed Au nanoparticles (AuNP) to upgrade porous sheets of RGO coated on micro-cantilevers towards VOC detection [138]. The sensors were selective for trimethylamine (TMA). The authors reported a lower influence of 100 ppmv humidity on the response of AuNP-RGO sensing layers $(0.4 \mathrm{~Hz})$ than for AuNP-GO layers $(2.8 \mathrm{~Hz})$. However, when extrapolating to $50 \% \mathrm{RH}$, as typically present in the environment, one gets a frequency shift of $\sim 40 \mathrm{~Hz}$,

\footnotetext{
$\overline{12}$ DDS stands for 4,4-diaminodiphenyl sulfone, and $\mathrm{MeOH}$ for methanol. The compound was already addressed in the first part of the review in the section "Specific interactions and their role in receptor sensitivity and selectivity".

${ }^{13}$ This class of materials could also be addressed in the next section, "Functionalization of specially devised receptor materials", due to some kind of regular free spaces inside. However, it has been included in the present paragraph because its members are one-element materials whose intrinsic regularity comes from the nature of the chemical bonding and from the symmetry it induces.

${ }^{14}$ Both values (detection limit and cross-sensitivity) inferred by the authors of the present review from the original data of the study [136].
} 
Fig. 5 Stacked chart of the $\mathrm{Cu}$ (II) complex for formaldehyde detection synthesized by Wang et al. Reproduced with the kind permission of Elsevier B.V. from reference [120]
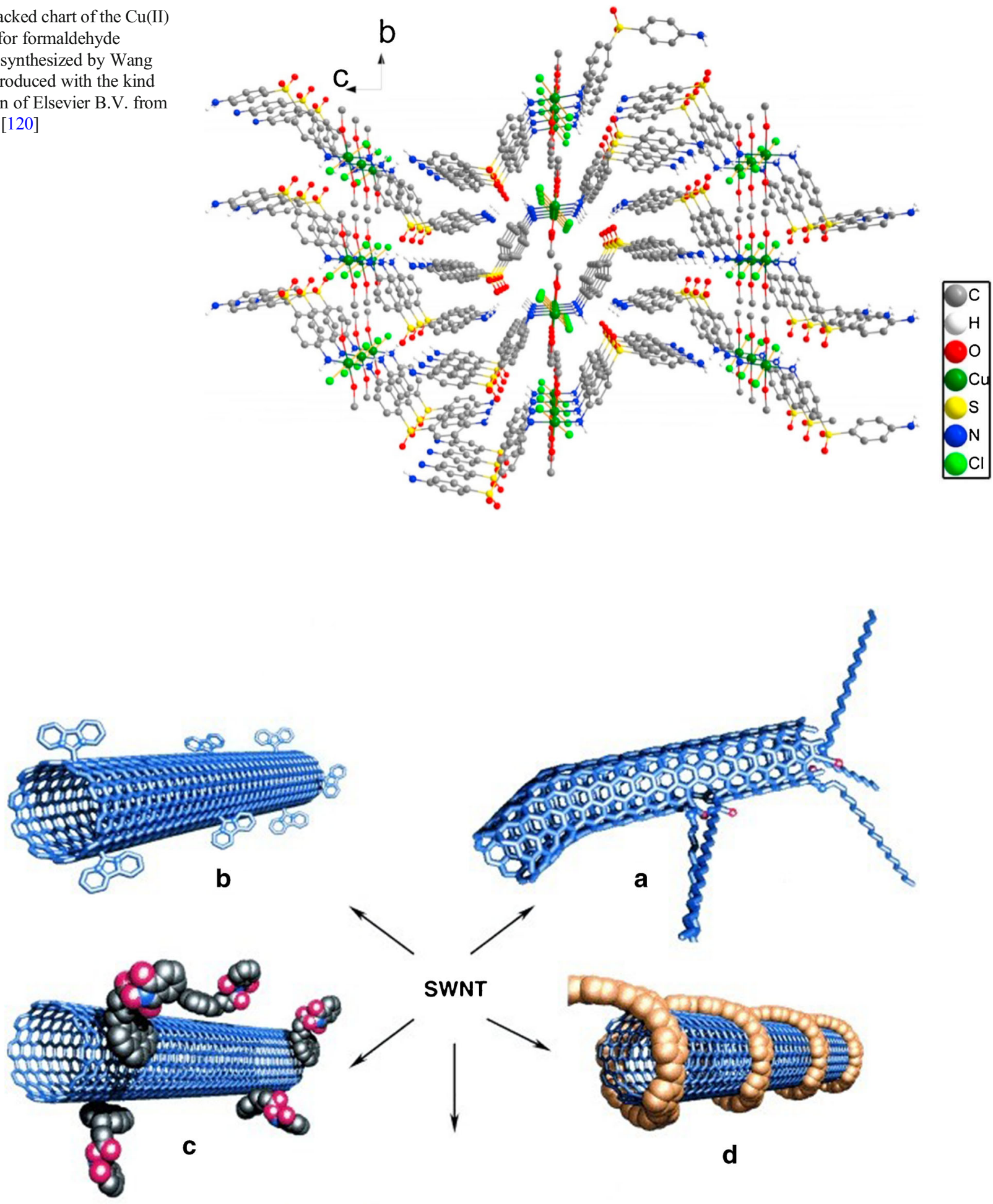

d

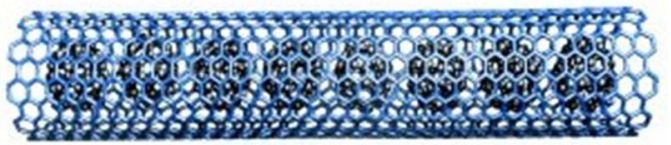

e

Fig. 6 "Different functionalization approaches for single-wall nanotubes (SWNT) of carbon. A) defect-group functionalization, B) covalent sidewall functionalization, C) non-covalent exohedral functionalization with surfactants, D) non-covalent exohedral functionalization with polymers, and $\mathbf{E}$ ) endohedral functionalization with, for example, C60. For methods $\mathrm{B} \pm \mathrm{E}$, the tubes are drawn in idealized fashion, but defects are found in real situations" (original caption). Reproduced with the kind permission of Wiley VCH from reference [132] 
which is five times larger than the response to 10 ppmv TMA (roughly the TLV-TWA for this analyte). Almost the same group of authors used carboxyl-functionalized AuNPs grown in situ on $\mathrm{RGO}$ for $\mathrm{NH}_{3}$ sensing with a similar cantilever [139]. The selectivity to ammonia was satisfactory at the evaluated concentration of $300 \mathrm{ppmv}$, but again one obtains sensor responses extrapolated to $50 \% \mathrm{RH}$ larger by an order of magnitude than TLVTWA of $\mathrm{NH}_{3}(\sim 30$ ppmv).

Functionalization of specially devised receptor materials. Two large categories are relevant here, the zeolites [140] and the porous coordinative polymers [141], usually addressed as metallo-organic frameworks (MOF). Except for natural zeolites, which are increasingly less used for gas sensing, these materials are synthesized to target the required properties for the desired application ${ }^{15}$. Often, established members of these families are functionalized and utilized as receptor materials. The zeolites are crystalline materials (aluminosilicates and similar ones) with regular nano and meso porosity, having a rigid three-dimensional (3D) structure [153]. Though encountered as natural mineral, for the purposes of gas sensing $[154,155]$ they are chemically synthesized [140] and coated on transducer devices [156]. Urbiztondo et al. tuned the properties of zeolites either through synthesis or by modifying commercial products through ion exchange before deposition as colloidal suspensions on cantilevers [157]. A detection limit of $\sim 0.5 \mathrm{ppmv}$ o-trinitrotoluene was obtained with the $\mathrm{Co}^{2+}$-BEA zeolite (for BEA zeolite see reference [156]). Using a La-modified AlPO-5 zeolite, Wang et al. measured $\mathrm{NH}_{3}$ concentrations down to $60 \mathrm{ppbv}$ [158]. The electrodeless Q-TSMR was read out in a wireless setup. A significant influence (about a factor 2) of the background humidity on the responses towards ammonia might limit the sensor applicability.

MOFs were devised to expand the capabilities of zeolites. In the MOF structure, metal ions or clusters are linked by organic ligands [143, 144, 159-163]. The MOFs became interesting for chemical sensing $[164,165]$ in general and for the particular case of gas sensing [166-169] due to their large specific area and selective gas adsorption, especially when functionalized [163, 170-172]. Lv et al. coated microcantilevers with Ni-MOF- $74^{16}$ and succeeded in sensitive detection of $\mathrm{CO}$ ( $\mathrm{LDL}=10 \mathrm{ppbv}$ ) in the absence of humidity. The same group of authors used home-synthesized MOF- $5{ }^{17}$ to detect aniline with an LDL lower than 1,4 ppmv, also with micro-cantilever transducers [174]. However, the

\footnotetext{
${ }^{15}$ The gas sensing is not the main target application of such materials, which are mostly used as molecular sieves [142] or for gas storage, chemical compound separation and heterogeneous catalysis [143-152].

16 The structure and names of some types of MOFs can be found in references [159, 173].

${ }^{17}$ MOF-5 is addressed, for example, by the references [143, 159].
}

cross-sensitivity to humidity was two orders of magnitude larger. Yamagiwa et al. reported the detection of VOCs with $\left[\mathrm{Cu}_{3}(\mathrm{BTC})_{2}\left(\mathrm{H}_{2} \mathrm{O}\right)_{3}\right] \cdot \mathrm{xH}_{2} \mathrm{O} \quad(\mathrm{BTC}=1,3,5$ benzenetricarboxylate) and $\left[\mathrm{Zn}_{4} \mathrm{O}(\mathrm{BDC})_{3}\right](\mathrm{BDC}=1,4-$ benzenedicarboxylate) [175]. The transducers employed were Q-TSMRs or silicon micro-cantilevers. The sensitivity enabled measurements in the TLV-TWA range for the selected analytes, but the selectivity, expressed through comparative absorption isotherms only, seems to have been rather poor. The influence of humidity was not addressed. He et al. synthesized a covalent organic framework (COF) through the Schiff base condensation of 1-(4,7-bis (4-aminophenyl)-1H-benzoimidazole-2yl)ethan-1-ol (BABE) with 1,3,6,8-tetrakis(4formylphenyl)pyrene (TFPy), which they abbreviated BABE-TFPy COF [176]. It was sensitive (LDL $\sim 1$ ppmv) to 2-chloroethyl ethyl sulphide (CEES), a mustard gas simulant. DFT calculations revealed evidence of a double hydrogen bond between the receptor material and the analyte (one between the $\mathrm{OH}$ group of $\mathrm{BABE}$ and thioether of CEES and another between the NH group of BABE and $\mathrm{Cl}$ of CEES), which provided good selectivity excepting humidity ${ }^{18}$. Wang et al. reported high sensitivity $(100 \mathrm{~Hz} / \mathrm{ppmv}, \mathrm{LDL} \sim 60 \mathrm{ppbv})$ towards ammonia for a Ladoped AlPO-5 framework (refer to [153] for the material) coated on TSMR transducers [158]. The logarithmic dependence of the calibration curve in the TLV-TWA range (25 ppmv) would not be a real issue, but the high crosssensitivity to humidity (rather doubling the response to ammonia) requires hardware/software compensation. General investigations on the kinetics of the gas uptake in ultra-microporous frameworks with fast SAW sensors were reported by Paschke et al. [177]. The authors showed the ability to infer the diffusion rates of gases in the addressed sensing materials.

\section{Composite, polymorph and unusual receptor materials} Composite receptor materials are customarily prepared from different classes of compounds with dissimilar properties. Amorphous materials, which do not stress the MST, can be mixed with rigid or less adhesive materials to obtain better sensor performance. Such an approach is even more relevant for dielectric or chemoresistive sensors, where the components of the sensing mixture can individually perform the reception and transduction functions. The composite film from cellulose acetate and a representative compound (1-nbutyl-2,3-dimethylimidazolium hexafluorophosphate) of

\footnotetext{
${ }^{18}$ Table S3 in the supporting information of reference [176] shows that the water vapor concentration in the cross-sensitivity test was $1400 \mathrm{ppm}$ while the CEES concentration was $22.6 \mathrm{ppm}$. Extrapolating the water sensitivity to $50 \%$ $\mathrm{RH}$ at $40{ }^{\circ} \mathrm{C}(\sim 20,000 \mathrm{ppm})$ will result in a response twice as large for background humidity as for CEES.
} 
GUMBOS are sensitive to VOCs and have been utilized as GGS with a TSMR transducer [178]. Lal and Tiwari demonstrated the suitability of poly(epichlorohydrin) (PECH) alkyd resin used as composite with nanaoclay for the selective recognition of chemical warfare agent (CWA) simulants (1chloro-2-[(2-chloroethyl)sulfanyl]ethane (SM) and DMMP [179]. In a more sophisticated approach, Chen et al. proposed a mixture consisting of MIP (methacrylic acid with ethylene glycol dimethacrylate copolymer imprinted with hexanal) and hydrophobic silica nanoparticles [180]. Besides high specificity for hexanal, the receptor exhibited low cross-sensitivity to humidity (visible on the dynamic response with humidity in background, but not certified in a dedicated experiment with incremental humidity). A GO and poly(styrene) composite was found to be sensitive to ammonia down to a few ppmv [181]. CGO/chitosan nanocomposites for amine vapors were reported by Zhang et al. [182]. Sensitivity of 2-5 Hz/ppmv with Q-TSMR transducers, LDL below 3 ppmv and rather low cross-sensitivity to other VOCs would make these sensors appropriate for amine detection, but the large influence of background humidity is a real issue. SAW transducers covered with $\mathrm{ZnO} / \mathrm{SiO}_{2}$ composite films were employed by Wang et al. to detect ammonia well below 10 ppmv. The sensing mechanism ascertained by the authors involves a charge transfer between the analyte and $\mathrm{ZnO}$ from the sensing layer, inducing an increase in the sensing layer conductivity, and by that, a change in the resonance frequency of the transducer ${ }^{19}$. This should explain the low cross-sensitivity to other tested gases. However, the influence of the background humidity on the sensor response was not discussed and the sensing mechanism was not experimentally proved. A protonated poly(ethylenimine)-graphene oxide (P-PEI-GO) nanocomposite thin film was deposited by dipping onto Q-TSMRs for humidity measurements by Tai et al. [183]. The sensitivity increased linearly with the number of coated layers, but was strongly nonlinear with respect to humidity. The sensing layer stability over 30 days was good.

The polymorph receptors consisted of several thin layers and were used to better ${ }^{20}$ attach the sensing material to the transducer surface. Biological layers (acetylcholinesterase [AChE]) immobilized on TSMRs previously coated with reduced graphene oxide (RGO) were successfully employed for gas sensing (CWA simulant DMMP) [184]. Figure 7 illustrates this bimorph sensing film. GO and $\mathrm{ZnO}$ layered receptors deposited by Yuan et al. on TSMRs showed increased sensitivity and fast response to humidity [185]. Almost the same group of authors reported on $\mathrm{RH}$ evaluation with $\mathrm{GO} /$ poly(ethyleneimine), with

\footnotetext{
19 This type of transduction was addressed in the first part of this review in the section "MSTs combining mass sensitivity with other types of sensing"

${ }^{20}$ Better in terms of adhesion, acoustic impedance matching, chemical compatibility and transducer protection against corrosive gases/vapor.
}

similar performance [186]. In order to strongly reduce the crosssensitivity to humidity of the poly(dopamine) (PDA) sensing layer for formaldehyde GGSs, Wang et al. prepared a superhydrophobic coating of PDA with polymerized $n$ octadecylsiloxane (PODS) nanostructures [187]. The bimorph receptor displayed good immunity to water vapor (contact angles to water larger than $140^{\circ}$ ). Its response towards $97 \% \mathrm{RH}$ was equal to the one to $5 \mathrm{ppmv} \mathrm{HCHO}$ only, preserving at the same time the HCHO sensitivity.

Ionic liquids have also been tested as sensing materials [188-190]. In the case of six imidazolium-based ionic liquids, good signals and specificity to ethanol were achieved [190]. Unfortunately, an inappropriate choice of the concentrations for humidity tests hid a huge cross-sensitivity to this analyte. Reference [188] demonstrates the discrimination of VOCs with high-temperature ionic liquids. Linear discriminant analysis (LDA) gave good results, but in the absence of humidity.

\section{Detection of enantiomers in the gas phase}

For analytes possessing chiral symmetry [191-193], discrimination of the enantiomers in gaseous mixtures is possible by using receptors with complementary chirality, as generally accounted for by molecular recognition principles [12, 80, 194-201]. The sensing materials employed to achieve this aim largely belong to classes already addressed above. They are either suitably chosen or specially devised [163, 202-204]. Making use of receptors based either on both enantiomers of Chirasil-Val derivatives [205, 206] or on cyclodextrin derivatives [207], Bodenhöfer et al. were able to selectively detect the enantiomers of amino acids and lactate, and the chiral gaseous anesthetics isoflurane, enflurane and desflurane, respectively. In all cases the transducer was a TSMR. These authors also evaluated the chiral discrimination factors ${ }^{21}$ of the receptors for the given analytes and the changes in the corresponding differences in the sorption free enthalpies, enthalpies and entropies $^{22}$. The detection specificity for the R (right/rechtus) and $\mathrm{S}$ (left/sinister) enantiomers of $N$-trifluoroacetyl-alanin methyl ester (N-TFA-Ala-Ome) with enantioselective receptors like (R, S)-octyl-Chirasil-Val is shown in Fig. 8. The exposure to racemic mixtures led to similar responses from both types of receptors. The enantiomeric discrimination factor was found to $\mathrm{be}^{23}$, in mean value, $\alpha_{\text {Sensor }}=1.6,3$ and the corresponding enantiomeric difference of the free enthalpy, $\Delta \Delta G^{0}=-1050 \mp 100 \mathrm{~J} /$ mol at $303 \mathrm{~K}$. A witness polydimethylsiloxane-coated TSMR had low and nonselective responses.

\footnotetext{
${ }^{21}$ The chiral discrimination factor is the ratio of the partition ratios of the enantiomers.

${ }^{22}$ This evaluation is possible by twice applying the equation 18 from the first part of the review.

${ }^{23}$ This value was obtained with the authors' definition, which consider the ratio of the sensor signals.
} 


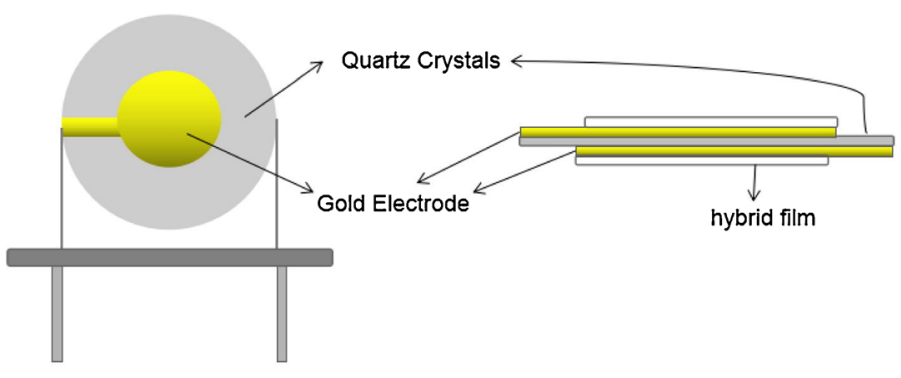

a

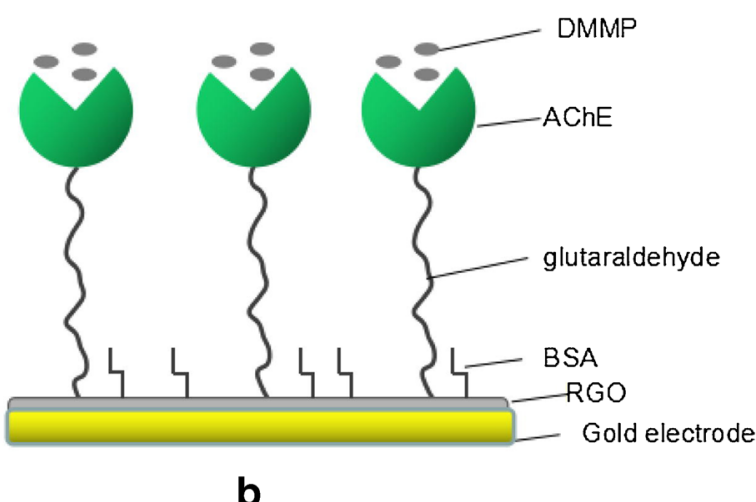

b

Fig. 7 Polymorph layered receptor based on reduced graphene oxide and acetylcholinesterase for the detection of dimethyl methyl phosphonate. a. The GGS sketch. b. The polymorph structure. Reproduced with the kind permission of Elsevier B.V. from reference [184]

The chiral discrimination ability of cyclodextrins has been extensively addressed in the literature, and the discrimination features (discrimination factors and differential thermodynamic parameters) acquired in gravimetric, calorimetric, chromatographic and nuclear magnetic resonance (NMR) experiments have been reported [2, 208-212]. Other cavitands, such as porphyrins, have also been considered as enantioselective receptors, either alone [213] or in association with other materials [214]. The advances in the synthesis/preparation of gassensing materials brought into play more diverse chiral receptors: macrocyclic arenes [100], metal-organic cages/ containers [204], metal-organic frameworks [163, 171] and thin film organic frameworks [145]. An extended review dedicated to the use of stereoregular chiral polymers for the separation of enantiomers (the liquid phase is also largely addressed therein) was elaborated by Shen and Okamoto [215].

\section{Gravimetric sensor systems}

The experimental results obtained with the selectivity strategies listed above did not fully confirm the optimistic expectations they raised because of the unspecific interactions which still contributed significantly to the sensor response. Therefore, gravimetric sensor systems (GSS), containing sensors with different specificity and, possibly, hardware for online data processing, have been seen as an effective way to address the selectivity problems that typically occur in the gas-sensing field. Since the variability in the responses of a sensor system is much greater than that coming from a single sensor, diverse approaches have been employed to build GSSs. The sensor array (SA) is the simplest system variant, in which either discrete sensors are assembled mechanically and electrically together, or integrated sensors are micro-machined on a single chip [216-219]. The degree of sensor signal processing at the array level is limited and usually lacks the chemical recognition facility. Electronic noses (EN) are instruments/devices encompassing analyte sampling stages, large and complex sensor arrays, readout and processing electronics, and chemometric software [43, 220-225]. They are able to detect and recognize odors and flavors and their individual chemical components in the gas phase. Several examples of gravimetric SAs reported in the literature are given in the following (the first of them for a historical perspective). The chemometric methods they employ will be reviewed in the section "Evaluating the performance of the gas sensors and sensor arrays". A discrete SA of TSMRs coated with six different poly(siloxanes) was employed by Hierlemann et al. to identify and quantify hazardous VOCs [226]. The values predicted by the array in test events (using partial least squares regression [PLSR]) were in good agreement with the true values. Kim et al. utilized polymer-coated cantilever arrays for qualitative and quantitative analysis of VOC mixtures [227]. The array output data were also evaluated with PLSR. Micro-machined cantilever arrays integrated on silicon chips using complementary metal-oxide-semiconductor (CMOS) technology were reported by Lange et al. [216]. They combine the advantage of direct signal amplification/processing with the increased discriminatory power enabled by multiple devices and sensing coatings. Thus, different features of the analytes and receptors are used simultaneously, and the reception specificity increases. Dickert et al. demonstrated the selective detection of 0-200 ppmv xylene in a common humidity background (up to $60 \% \mathrm{RH}$ ) with Q-TSMR arrays coated with compounds providing molecular complementarity to the targeted analytes [228]. The hardware sensing system was supported by MDA (PLSR) and artificial neural networks (ANN). The influence of humidity was practically rejected by the numerical algorithms. Discrete Love-wave sensor arrays coated with selected polymers (three poly(siloxanes), poly(ethyleneimine), poly(epichlorohydrin) and Carbowax) enabled the sensitive detection of DMMP (down to 40 ppbv) and good CWA discrimination by PCA combined with probabilistic neural networks (PNN) [229]. No false assignations occurred among the reported events. Roughly the same approach was employed by Senesac et al. for 11 

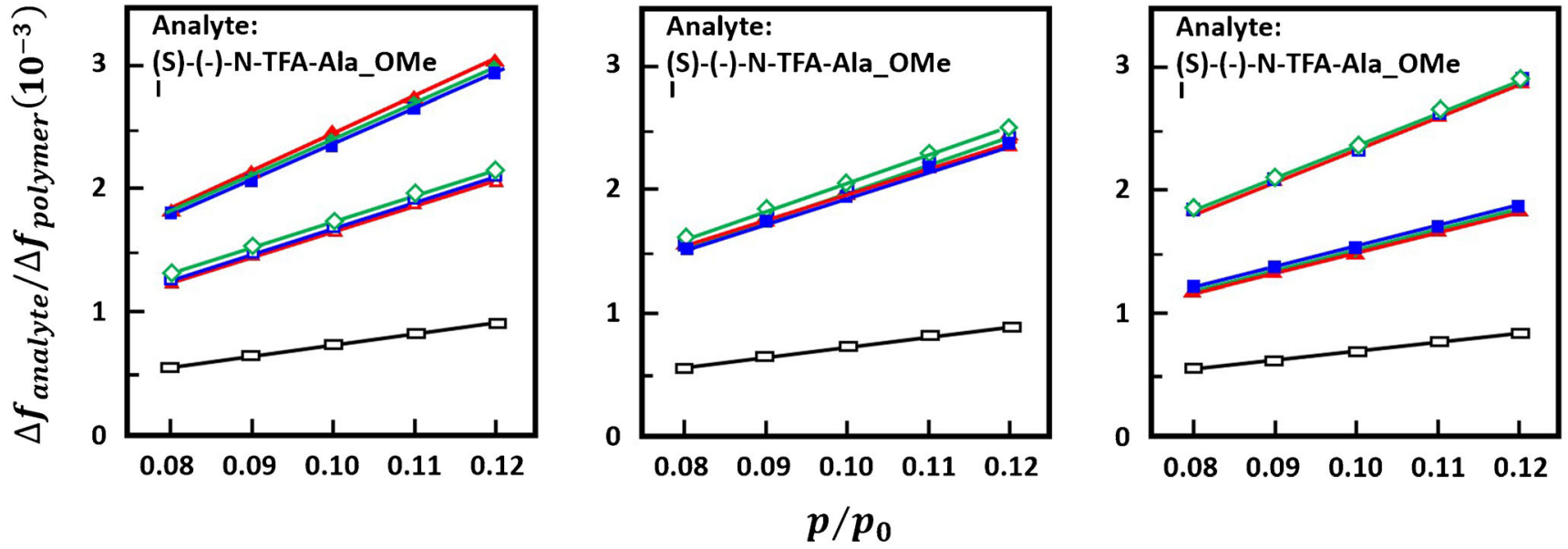

\begin{tabular}{|c|c|c|c|c|c|c|c|}
\hline Analyte:: & Polydimehylsiloxane & $(S)-0$ & $\mathrm{yl}-\mathrm{Cr}$ & jil-Val & $(R)-0$ & yl-Ch & sil-Val \\
\hline Sensor index: & $\begin{array}{c}\overrightarrow{S E}-30 \\
\end{array}$ & $\overrightarrow{s 2}$ & s4 & $\overrightarrow{\text { S5 }}$ & $\begin{array}{l}\text { R1 } \\
\text { Re }\end{array}$ & $\begin{array}{l}\triangle \\
\mathrm{R} 2\end{array}$ & $\widetilde{\curvearrowright}$ \\
\hline
\end{tabular}

Fig. 8 "Normalized (with regard to the frequency shift due to polymer deposition) TSMR responses to different concentrations (p/p0: adjusted pressure with respect to the saturation vapor pressure at $298 \mathrm{~K}$ ) of (R)-, (S)- and racemic $N$-trifluoroacetyl-alanin methyl ester. The responses of three (R)- (open symbols), three (S)- sensors (filled symbols) and one SE-

inorganic and organic vapors and gases [230]. The responses of the 10 cantilevers in the array were processed by a backpropagation (BP) ANN. A comparison of gravimetric and chemoresistive SAs based on polycyclic aromatic hydrocarbons with different side groups was reported by Bachar et al. [231]. Both types of arrays provided consistent results when assisted by PCA and discriminant factor analysis (DFA). Lu et al. reported on microfabricated FBAR sensor arrays coated with cavitands (calix[8]arene, porphyrin, $\beta$-cyclodextrin, cucurbit[8] uril) for selective VOC detection [232]. The authors assessed the suitability of their devices for an integrated electronic nose, but they did not fabricate the EN or use MDA in their investigation. The exposure to the analytes (chloroform, acetone, methanol, hexane, etc.) was performed at very high concentrations (10\% to $100 \%$ from each saturation vapor pressure) without background humidity. Though nice, the results seem not to be relevant for the conditions encountered in real applications. In a different approach, Mascini et al. demonstrated the possibility to "tailor" sensors for gas-sensing arrays using the virtual screening of a large database of tripeptides (8000 elements) in virtual interaction (molecular docking simulations) with VOCs (58 vapors) from five chemical classes [57]. Then, using a combinatorial method, 120 tripeptides with the highest interaction specificity were further employed to generate $\sim 7900$

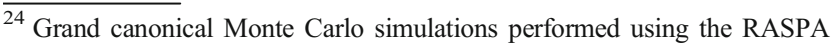
software package [233]

${ }^{25}$ The optimization uses Kullback-Leibler divergence evaluations [235].
}

30 sensor are displayed. Sensor index is given at the bottom" (original caption). Reproduced (the remake was necessary due to reduced resolution of original figure, whose faithful copy is) after [206]. The use of the figure was allowed by Springer Nature

virtual tetrapeptides, from which five were selected, prepared and covalently attached to gold nanoparticles and coated on TSMRs. The data outputted by the real arrays during gas exposure were evaluated in the PCA frame, confirming the good gas-sensing performance suggested by the design.

Based on atomistic molecular simulations ${ }^{24}$, Gustafson and Wilmer estimated the best choice among nine potential MOF receptors for an array theoretically designed to discriminate and recognize different target gases [234]. To illustrate the approach, $\mathrm{CH}_{4}, \mathrm{~N}_{2}$ and $\mathrm{O}_{2}$ were selected. The aim was to reduce the effort spent in trial-and-error experiments and to improve the host-guest matching of the analyte-receptor pair, on the one hand, and to reduce the dimensionality ${ }^{25}$ of the array, on the other hand. The SA was numerically tuned for $\mathrm{CH}_{4}$ detection, but any target analyte is in principle eligible. Complementing somewhat the virtual screening of receptor materials, Speller et al. devised a hardware virtual sensor array whose virtual sensors were the overtone responses of single Q-TSMR GGSs [236, 237]. A virtual sensor array for VOCs was obtained by Zeng et al., modulating the temperature of a film bulk acoustic wave (FBAW) transducer covered with self-assembled organic films [238]. The exposure events to the same analyte at different partial pressures appeared as straight lines in the first two principal components (PC). Dissimilar analytes were separated mainly along PC1 and clustered very well when classified with LDA. Chen et al. realized a SAW virtual array, adding a chromatographic-like column to a SAW sensor and evaluating the retention times 
for different analytes specific to lung cancer [239]. A very ingenious paper cantilever array functionalized with polymers, operating in deflection mode and with a visual readout, was devised and tested by Fraiwan et al. [240]. The LDA canonical score plot proved the complete separation of the clusters for acetone, methanol, ethanol and tetrahydrofuran. Many other authors report the successful use of more or less conventional GGS arrays to solve analytical problems in gas detection: ionic liquids Q-TSMRs for VOCs [241-244] or for explosive vapors/gases [245], $\mathrm{SiO}_{2}$-NP functionalized with organic materials Q-TSMRs for breath analysis [246], AuNP-peptide Q-TSMRs for food aroma detection [51], poly(acrylic acid) MIP on Q-TSMRs for aldehyde [247] and organic acids [248] in body odor, peptide-modified ZnO-NP on Q-TSMRs for organic VOCs [249], biomimetic MIPs on Q-TSMRs for terpenes from herbs [250], GO and $N$-substituted pyrrole derivative-based films on Q-TSMRs for toxic gases $\left(\mathrm{CO}, \mathrm{NH}_{3}\right.$ and $\left.\mathrm{NO}_{2}\right)$ [251], metallo-porphyrins and AuNPpeptide on Q-TSMRs for chocolate quality control, [103], SWCNT/organic materials on Q-TSMRs for ambient air composition (as example) [252], porphyrin Q-TSMRs for vapor released by microorganisms [104], polymer films on Si cantilevers for VOCs, $[253,254]$ and MIP on SAW for CWA detection [255]. In order to increase the accuracy of gas sensing, arrays of GGSs together with other types of gas sensors have been devised and tested. They will not be fully addressed in this review, but examples include integrated microcantilevers with micro-calorimeters and capacitors [256-258], Q-TSMRs with chemo-resistors [231, 259], SAW, Q-TSMRs and silica optical fiber [260]. An extended overview is available in the table as Annex.

The studies in the field of EN are quite old, some of them resulting in commercial instrumentation [220]. A modular hybrid EN (MOSES 11) was devised by Ulmer et al., including QTSMRs together with metal oxide, electrochemical and calorimetric sensors [261]. It uses an Agilent/HP 7694 headspace autosampler when required. In evaluation tests, the VOC mixtures, coffee and tobacco flavors and other odors were well separated and recognized. McGill et al. reported an EN (NRLSAWRHINO) based on SAW sensing devices coated with functionalized polymers for CWA [262]. The instrument employs, as a first stage, a trap-and-purge gas-solid chromatographic column. The discrimination and clustering in the first two principal components of the nerve and blister chemical agents, with various interferents in the background, was error-free. An EN using a chromatographic column and poly(isobutylene) virtual SAW array was developed by Chen et al. to detect lung cancer through 11 marker VOCs [239]. The postprocessing of the signals was conducted using a BP-ANN which delivered a graphical output towards an image recognition approach. There was no incorrect disease identification, but one ill and one healthy person (from 5 ill and 5 healthy) were categorized as suspects only. Starting from an eight-cantilever array functionalized with polymers,
Lang et al. [263] built an EN for fragrance characterization and identification of disease-specific odors. The transducers were operated in proportional bending mode, induced by swelling of the sensing layers, with optical readout. The acquisition of beam deflection amplitudes at five successive time points during the exposure increased the dimension of the array output to 40 . The nose was able to separate very well, in the plane of the first two principal components, the common VOCs, different natural scents and VOCs specific to diseases. With a cantilever EN employing ANN, Leis et al. were able to detect DMMP in ppbv range in ternary mixtures with water and ethanol in ppmv ranges [264]. Although scientifically relevant, this performance would not be enough in a practical application, where the humidity is much higher. Fernandes et al. used a SAW-EN to detect VOCs [265]. The PCA discrimination for equal analyte concentrations (50 ppmv) in mixtures was good, but when the concentrations were spread over large ranges, the clusters overlapped. A chromatographic column connected to Q-TSMRs was utilized by Rivai et al. to construct an EN for VOCs and odors [266]. Electronic signal conditioning and ANN pattern recognition allowed the authors to map a large number of odors and to discern more the 20 of them in a 2D PCA plot. Magna et al. utilized gas specificity induced by the preparation route of porphyrin-functionalized $\mathrm{ZnO}$ (coated on Q-TSMRs) to enable selective operation of an EN designed to detect VOCs [94]. Some studies addressing both sensor SA and EN were already considered in the SA paragraph above, while reports on practical applications with EN will be referred to in the dedicated section "Targeted analytes and applications". Pertinent reviews devoted to bioelectronic noses, related biomaterials and artificial olfaction were published not too long ago by Wasilewski et al. [46, 224] and Barbosa et al. [45]. There are also studies investigating accessories for SA and EN as pre-concentration units $[267,268]$ or ASIC interfaces for TSMRs and micro-resistors $(\mu \mathrm{R})$ [269]. In the literature, reviews can be found dedicated to gravimetric sensors, SA and EN based on given types of MSTs [219, 222, 270, 271] for instance.

\section{Deposition methods for sensing layers on MSTs}

The most appropriate receptors for MSTs are thin sensing layers firmly attached to the transducer surface. The thickness should fit the MST operating requirements addressed in the section "Mass-sensitive transducers" of the first review part. Therefore, coating procedures for thin films are largely employed, and are well surveyed by the general literature [272-279]. A schematic overview of several surface coating 
methods as proposed by Oluwatosin Abegunde et al. is included in Fig. 9 [272].

A few of these will be referred to below, with additional references. A complete overview of the most commonly employed methods for the manufacture of GGSs is contained in Annex, where they are reported together with sensor performance.

Dip and drop coating/casting are the simplest coating methods, but have lower precision and result in large spreads of the morphology and geometric parameters of the deposited layers [280]. Therefore, they are mainly utilized in the incipient phases of an investigation. Automated variants of the methods can enable the manufacture of less demanding films. Das et al. [281] reported a process involving dipping followed by polymerization to coat castor oil with different amounts of benzoyl peroxide on TSMRs sensitive to aliphatic amine vapors. Ayad et al. used drop casting to prepare chitosan/ poly(aniline) nanofibers for TSMR detection of methyl/ dimethyl amine and ethanol [282]. Poly(aniline) emeraldine salt thin films doped with different acids were also deposited by dip coating on TSMRs as VOC sensors [283]. Figure 10 shows the morphology of drop-coated Q-TSNRs with hexanal MIPs and their composite with hydrophobic silica, as prepared by Chen et al. [180].

Spray coating, inkjet printing, electrospray coating, spin coating and electrospinning enable the covering of surfaces with materials solubilized or suspended as powders in carrier solvents. Through spray coating, solutions/suspensions are transformed into aerosol flow by a nozzle [284-286]. Spray coating is very popular for polymeric coating of TSMRs $[185,186]$. Sometimes a manual version, airbrush coating, is preferred [243, 287]. The morphology of a layer family (GO, PANI, and $\mathrm{GO} / \mathrm{SnO}_{2} / \mathrm{PANI}$ ) deposited by airbrush spray coating on an Ag Q-TSMR electrode by Zhang et al. is shown in Fig. 11 [287]. Humidity evaluation with this layer is addressed in the paragraph "Humidity" below. Inkjet printing is the modern version of spray coating inspired by paper printing technologies [288]. It allows for better control of the coated area and film thickness and porosity, being suitable for small devices where precision is a key requirement. Figure 12 shows the SEM image of carboxyl groupfunctionalized mesoporous silica nanoparticles (C-MSNs) inkjet-printed on the active area of a Si micro-cantilever for ammonia detection [289]. Spin coating combines drop casting with high-speed rotation of the substrate. Because of centrifugation, rather uniform and smooth layers can be produced. Electrospray coating $[290,291]$ and electrospinning [292, 293] use electrical fields to improve the deposition and to produce fiber layers/structures. Jia et al. deposited nanofibers with different specific surface areas on Q-TSMRs from poly(styrene-block-maleic acid) by electrospinning [294] and poly(acrylic acid) (PAA) by electrospray/electrospinning for ammonia detection [295] (see Fig. 13). Electro-netting and electrospinning were employed by Wang et al. to coat TSMRs with two-dimensional layers of pure and $\mathrm{NaCl}-$ doped poly(acrylic acid) for trimethylamine detection [296]

Self-assembly and Langmuir-Blodgett techniques enable the formation/deposition of molecular monolayers, mainly of organic compounds, through supramolecular processes [297-301]. The methods have also been used for the preparation of gas-sensing monolayers [102]. Xie at al. reported $\mathrm{ZnO}$ colloid spheres for alcohol detection prepared via selfassembly [302]. Figure 14 shows the self-assembly of a hyper-branched polymer on a Si micro-cantilever for DMMP detection prepared by Guo et al. [303].

Chemo-physical methods The methods included here are numerous [273]: chemical vapor deposition (CVD),

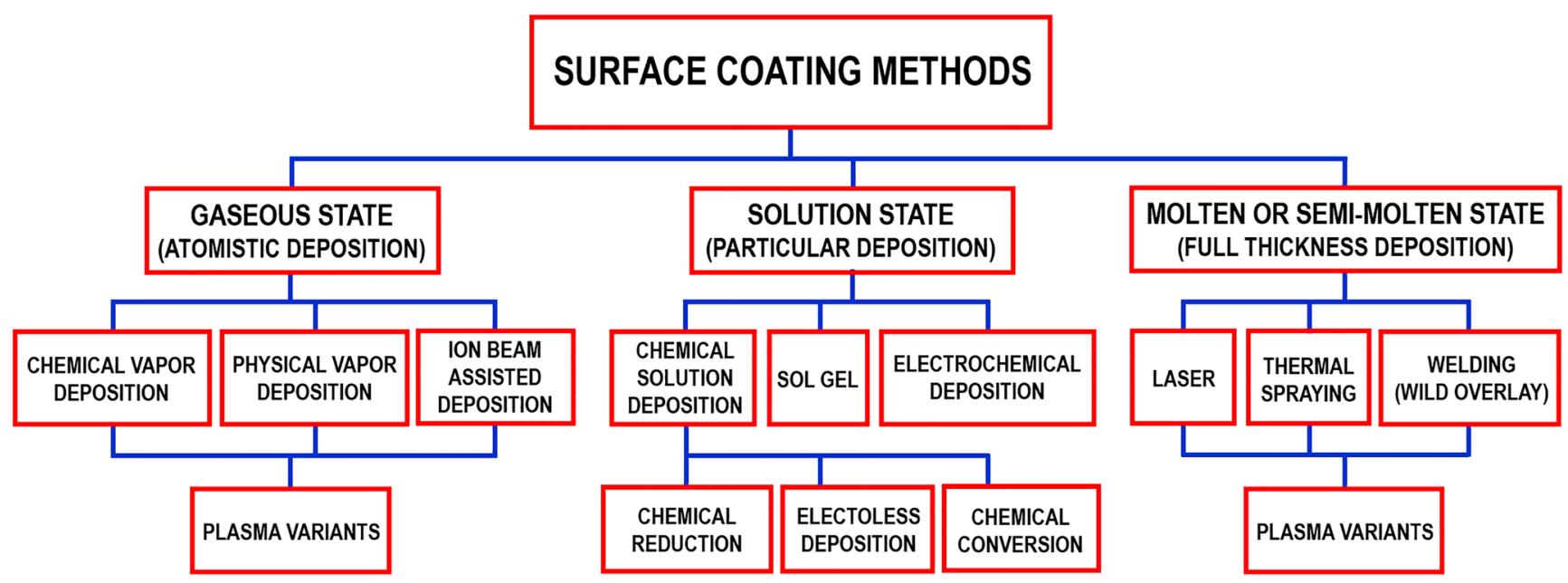

Fig. 9 Schematic overview of several surface coating methods. Reproduced (the remake was necessary due to reduced resolution of original figure, whose faithful copy is) with the kind permission of the authors and AIMS Press from reference [272] 
Fig. 10 Hexanal MIP (a) and hydrophobic hexanal MIP-SiO ${ }_{2}$ NPs composite (b) deposited by drop coating. The inset pictures indicate the water contact angles (WCA) of the films. Reproduced with the kind permission of Elsevier B.V. from reference [180]
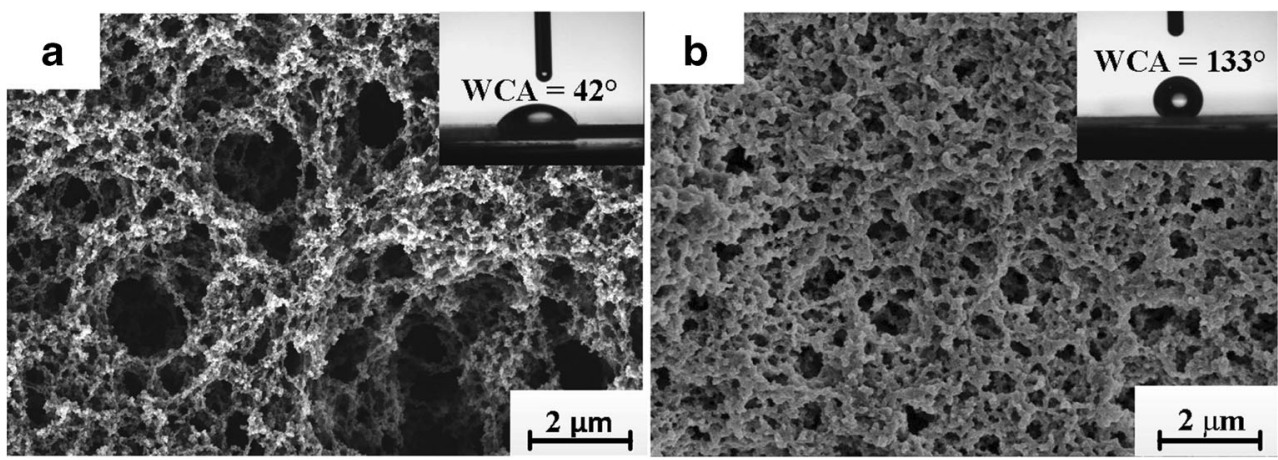

plasma-enhanced chemical vapor deposition (PECVD), sputtering (with the variants direct current [304], radiofrequency, reactive and magnetron), thermal evaporation (TE) (with the electron gun/beam variant), atomic layer deposition (ALD) [305-308], molecular beam epitaxy (MBE) [284], pulsed laser vaporization and deposition (PLD) [309], and other laser processing techniques [310, 311]. They are suitable for inorganic thin and very thin layers. Some of them, CVD, PECVD and TE, can also be employed for soft organic materials [312].
Electrochemical methods Almost all the MSTs contain metallic electrode structures. Certain types of electrodes are appropriate for electrochemical deposition of sensing materials, or at least for electrochemical polymerization. Because their electrodes cover a compact device area, TSMRs have been mostly used in electrochemically assisted processes. Pristine and Pd-doped $\mathrm{ZnO}$ nanorods have been electrochemically grown (in two steps) on quartz TSMRs and used for volatile organic compound (VOCs) detection at room temperature [313].

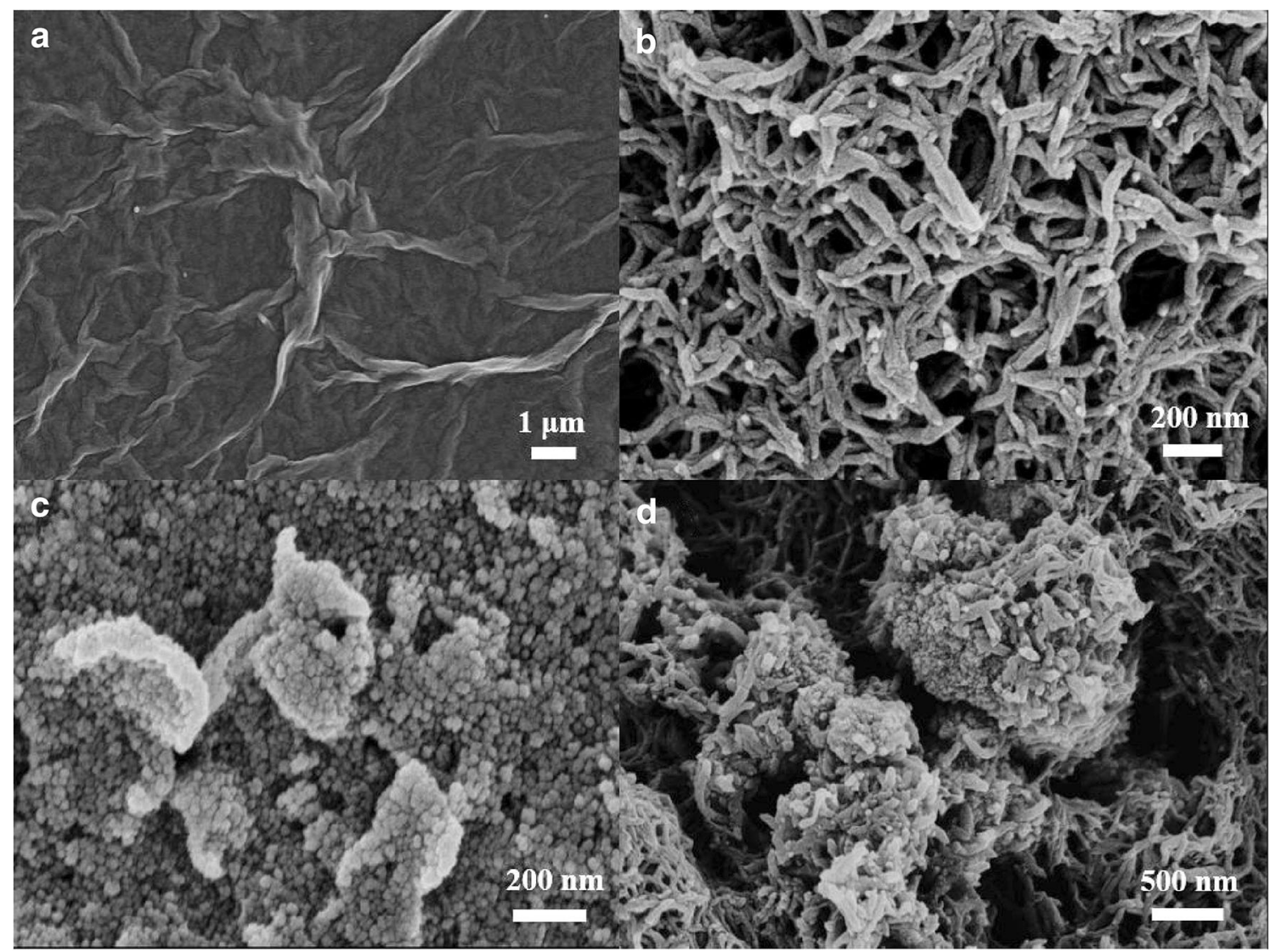

Fig. 11 The morphology of GO (a), PANI (b) and GO/SnO $2 /$ PANI (c), (d) deposited by airbrush spray coating on Ag Q-TSMR electrode. Reproduced with the kind permission of Elsevier B.V. from reference [287] 
Fig. 12 SEM image of carboxyl group-functionalized mesoporous silica nanoparticles (C-MSNs) inkjet-printed on the active area of a Si micro-cantilever. Reproduced with the kind permission of Elsevier B.V. from reference [289]

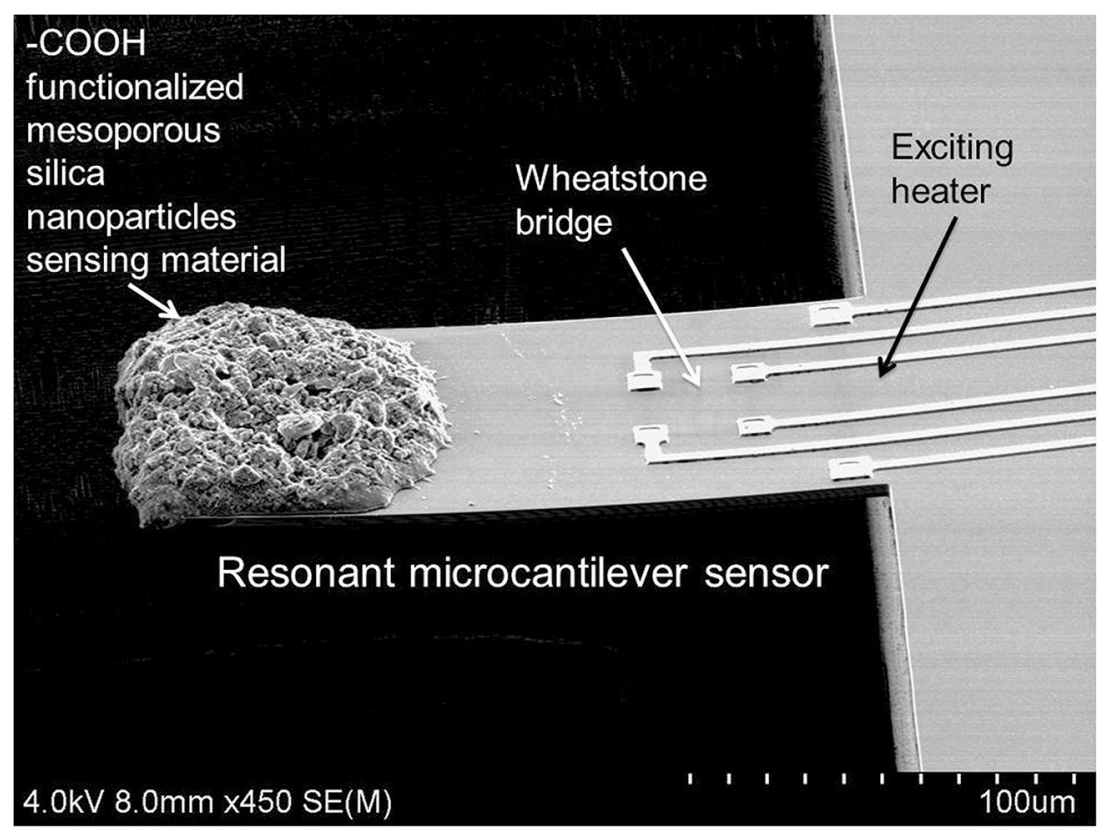

Nonconventional deposition approaches Many sensing layers, based on composite materials or stacking layers of dissimilar structure, have also been considered for gas sensing. In order to coat layers with complicated morphologies on MST surfaces, researchers have been pushed to find original deposition procedures. For example, Yan et al. employed a biosynthetic approach to prepare poly(dopamine) nanotubes sensitive to $\mathrm{CHOH}$ [314]. Sabri et al. produced poly(styrene) (PS) monodispersed nanosphere monolayers (MNM) on Q-TSMRs by dispersion polymerization, which were then coated by electron beam evaporation with $\mathrm{Au}$ and $\mathrm{Ag}$ thin films to obtain (Au-MNM) and Ag (Ag-MNM) nanostructures sensitive
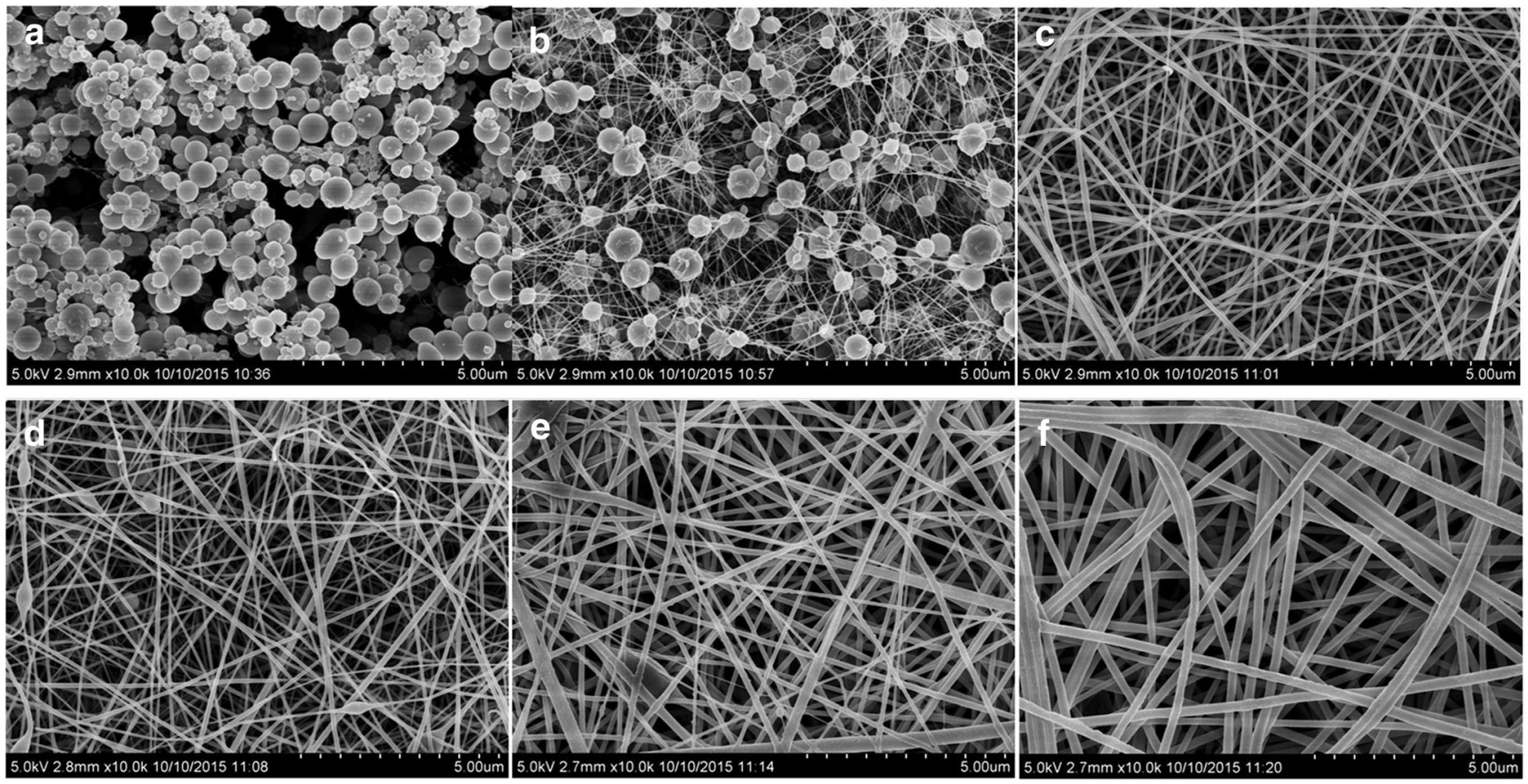

Fig. 13 FE-SEM of PAA layers deposited by electrospinning/electrospray: NPs (a), bead and string (b), fibers (c-f) from different solutions. Reproduced with the kind permission of Elsevier B.V. from reference [295] 


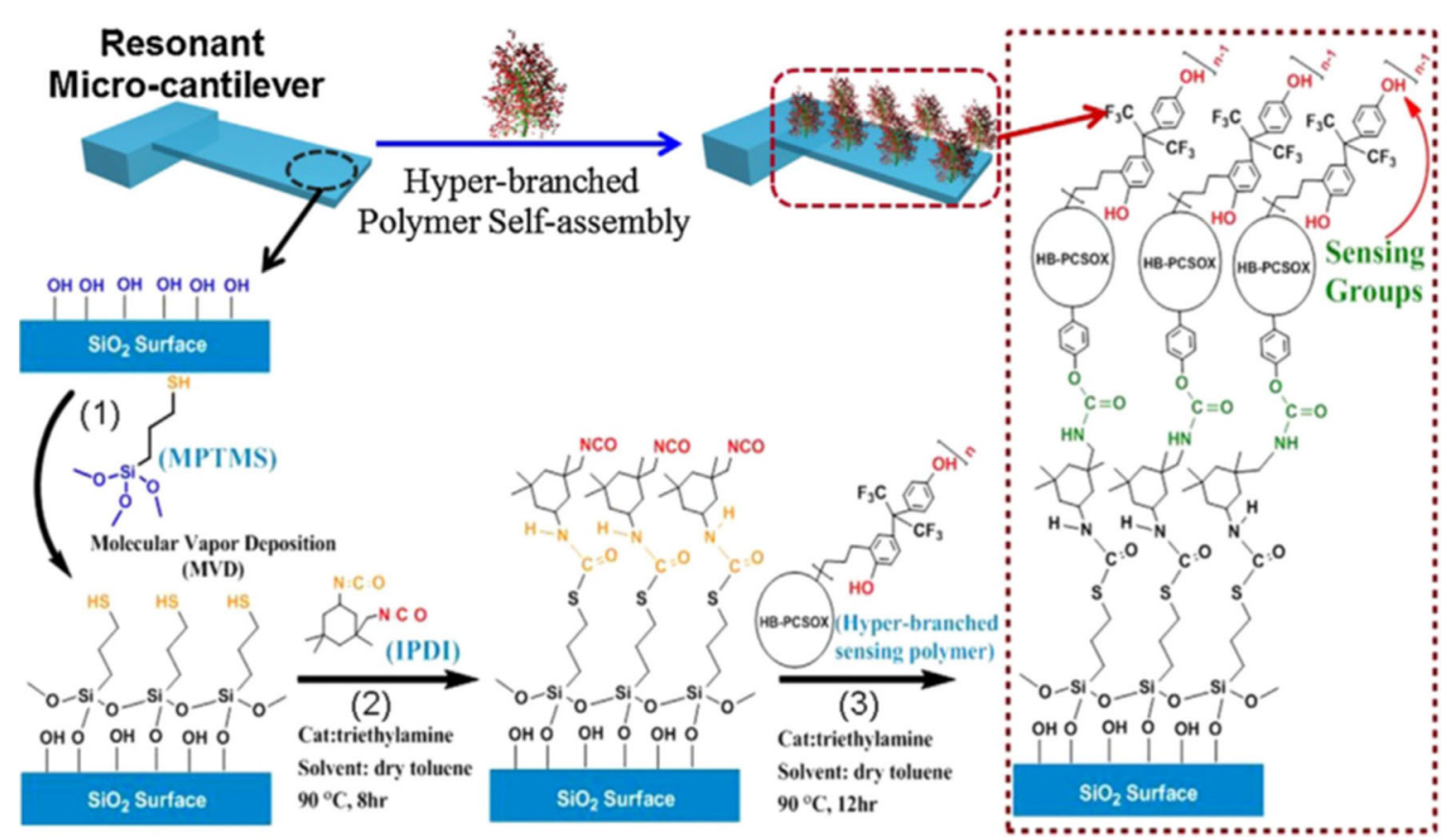

Fig. 14 Batch self-assembly of a hyper-branched polymer on a Si micro-cantilever. Reproduced with the kind permission of Elsevier B.V. from reference [303]

to Hg vapor [315]. Figure15 displays the SEM images of the films addressed above.

\section{Evaluating the performance of the gas sensors and sensor arrays ${ }^{26}$}

The data provided by the GSs or SAs have very limited utility without appropriate handling. As ascertained in the paragraph "The performance of GGSs" of the first review part, in the case of individual sensors, this mainly involves the determination of the calibration curves (see the paragraph "Univariate and multivariate calibration" of this section), sensitivity, detection limits, response/recovery times and cross-sensitivity (the sensitivity towards gases/vapor other than the targeted one) [316]. On the other hand, SAs acquire large amounts of experimental data which need to be converted into chemical information such as chemical nature and concentration of the components in the gaseous samples. The best way to accomplish this task is to appeal to appropriate chemometric methods, which use mathematical/statistical approaches for data processing and, as such, significantly increase the accuracy of the information provided [316-330]. Kiralj and Ferreira give a nice and unique "etymological, linguistic, and bibliometric" perspective on chemometrics until 2006 [331]. Chemometric software packages such as Unscrambler ${ }^{\circledR}$ (Camo Analytics, https://www.

\footnotetext{
${ }^{26}$ This topic exceeds the frame of the present review and is kept at a minimal extent. The formal terminology and the mathematical rigor were not the main priority of this section. Complementary information about the considered topics can be found in the addressed references.
}

camo.com/unscrambler/), SIMCA $^{\circledR}$ (Umetrics/ SartoriusStedim Biotech https://umetrics.com/products/simca) and PLS Toolbox ${ }^{\circledR}$ (EigenVector Research Inc. http:// eigenvector.com/software/pls-toolbox/) are available on the market. Not all chemometric methods are equally utilized in the field of GGSs, as obvious from the content of the articles published by different authors. The most relevant ones are briefly presented below and summarized in Table 1 .

\section{The structure and properties of the data provided by SAs}

The bare output of the GGSs, usually delivered as electrical signals, is preprocessed by the readout electronics or hardware stages, which provide amplification, noise reduction and the conversion to digital signals. The obtained digital data can undergo simple upgrade (coding) as scaling (division by a value representative for the set, like statistical dispersion, matrix norm, etc.), centering (shifting of the mean to zero) [323], or even smoothening and derivation [347]. More complex data conditioning, such as Fourier transform or wavelet transform, often related to the spectroscopic analytical methods, are less commonly employed in gravimetric gas sensing.

In the SA evaluation approach, the data are obtained from series of $M$ gas exposures (events) under the influence of $I$ different conditions (factors) $\left\{f_{i}\right\}$. The individual outputs of the $N$ sensors in the array are expressed as features, $\left\{x_{n}\right\}$. During an event, one has/sets a certain value for each factor and, correspondingly, one obtains a value for each feature. 

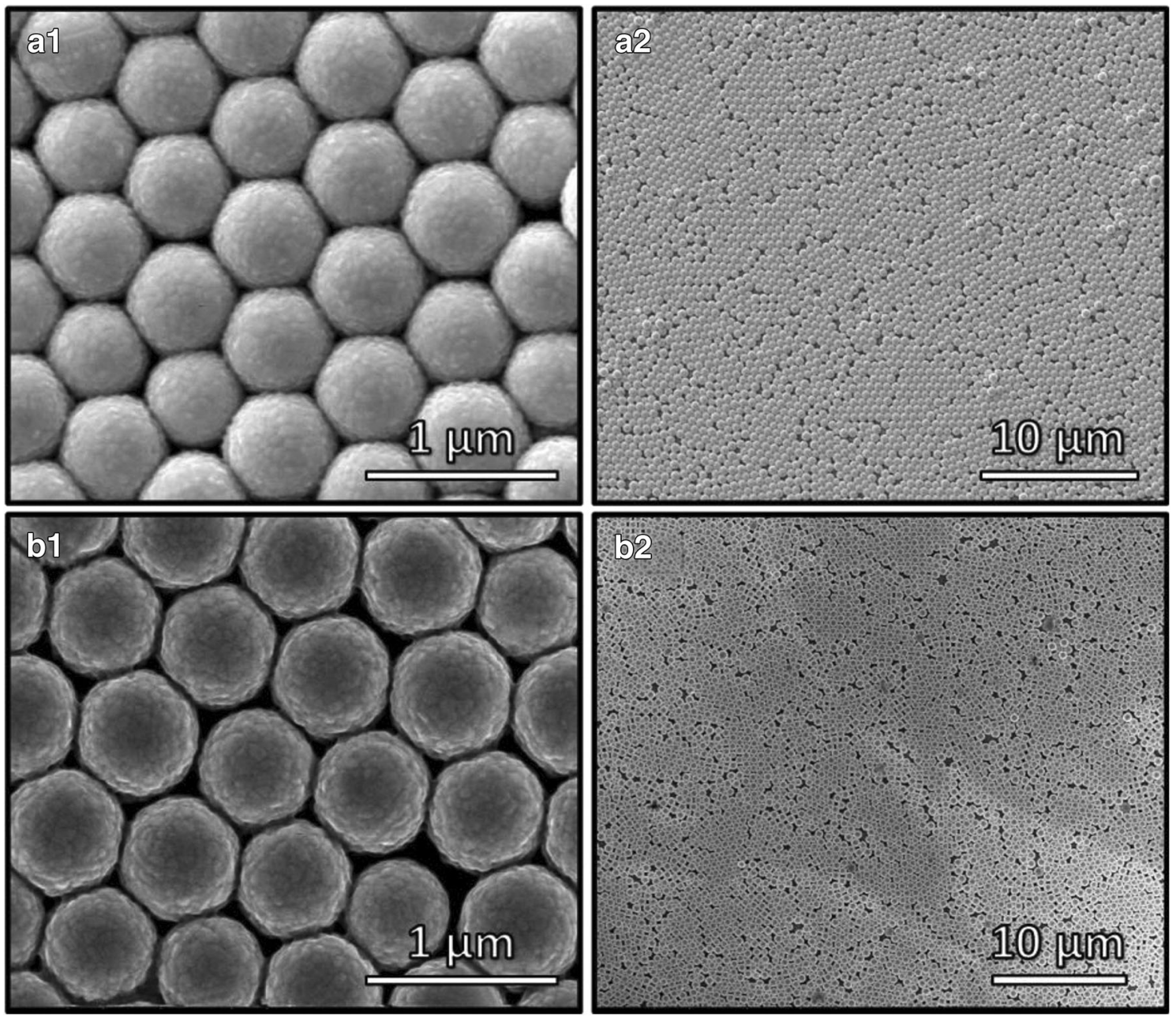

Fig. 15 "SEM images representing (a1) close-packed Au-MNM, (a2) surface coverage of Au-MNM on the Ti electrode of QCM transducer, (b1) close-packed Ag-MNM, and (b2) surface coverage of Ag-MNM on

The raw factors have a chemical or physical nature (the concentrations of different gases and vapor in the test mixture, and the temperature, pressure, flow rate of the test mixture, respectively), while the features usually lose their physical identity in the preprocessing stages, becoming abstract numbers. Actually, the factors can also undergo some coding procedures when handled by different chemometric methods. From an algebraic point of view, the factors are independent variables, while the features are dependent ones (for linear algebra concepts please consider appropriate textbooks, like those of Strang [348], or Anton and Rorres [349]). The features belong to an $N$-dimensional space whose coordinates are not linearly independent because of some similarities in the sensor responses [350]. This complicates the estimation of the distance between events in the feature space and the analysis of the possible relationships among them. The SA data are usually organized in a data matrix [351] $\boldsymbol{X}=\left\{x_{m, n}\right\}$, where $m \in[1, M]$ and $n \in[1, N]$ index the events and the features, respectively. For instance, the matrix element $x_{m, n}$ reflects the Ti electrode of QCM transducer" (original caption). Reproduced with the kind permission of ACS Publications from reference [315]

the output of the sensor number $n$ when exposed to the conditions of the event $m$. Each row $m$ of the data matrix is a set of features (scores) recorded for a given event, implicitly containing the dependence on the factors acting during that event. It can be regarded as a row matrix (raw score vector) $\boldsymbol{x}_{m}$, with the dimension $N$. A column of $\boldsymbol{X}$, in its turn, is a column vector (column matrix) $\boldsymbol{x}_{n}$ in the event space bearing the information about all $M$ events corresponding to the feature $n$ (that is, all the information outputted by the SA sensor with the number $n$ ). The larger the variability ${ }^{27}$ among the components of $\boldsymbol{x}_{n}$, the more information about the set of events is carried by the feature $n$ with respect to the other features. In order to compare the data on different columns of $\boldsymbol{X}$, they must be normalized (divided by their matrix norm, that is, by the square root of the sum of their squares), because different sensors can give different types/ranges of responses. Care is required when

\footnotetext{
${ }^{27}$ The imprecise term "variability" is used here, because the "variance" of the data has not been addressed here.
} 


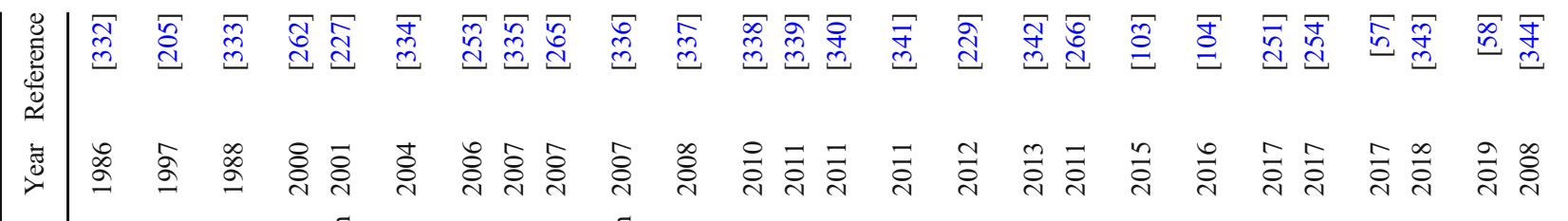

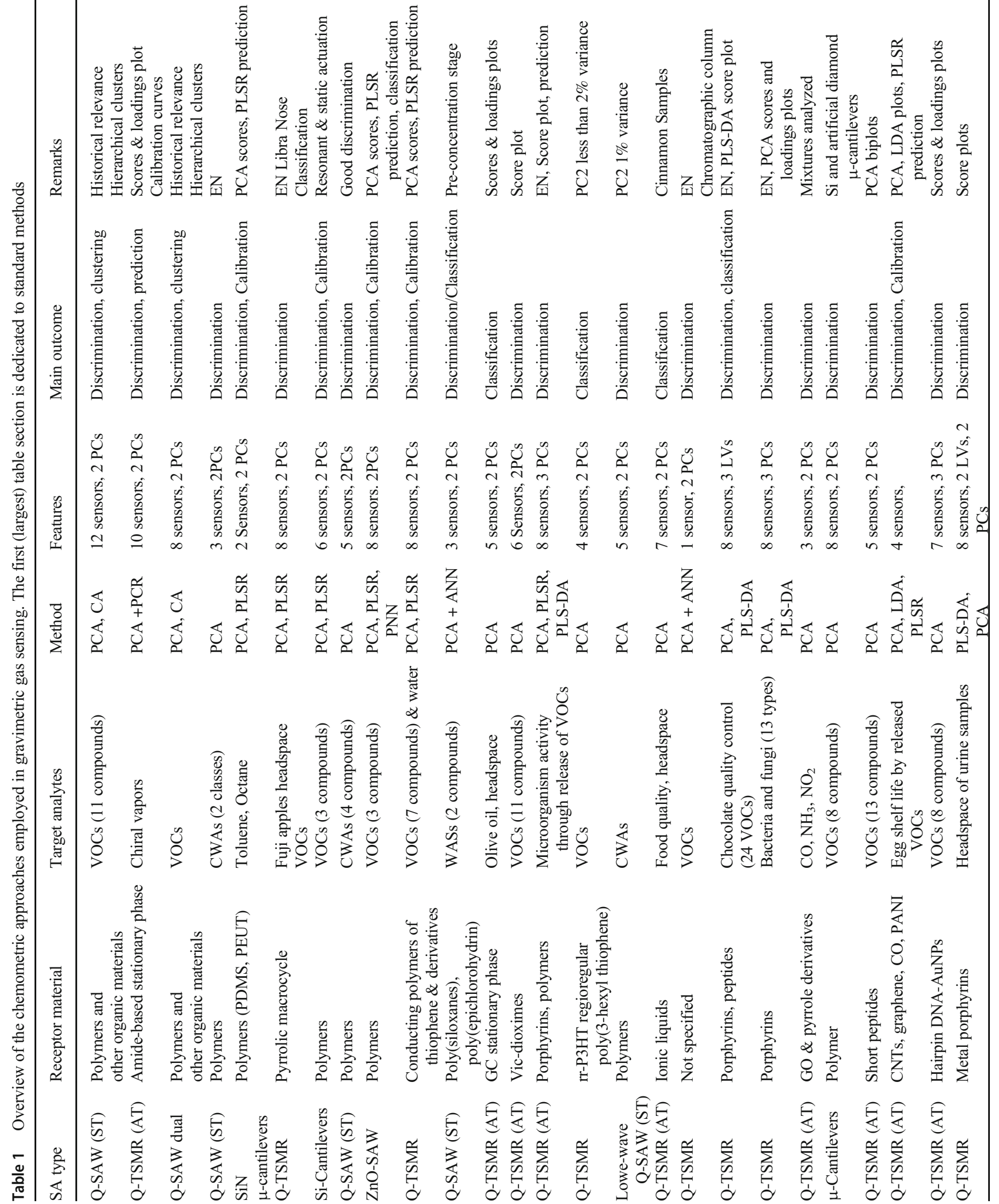


identifying the meaning of the notations in the data reported in the literature. It is possible that the same character has two or more meanings. For instance, " $x$ " could be used for independent variables in the experimental design and for dependent variables in PCA (see below). In following, this double use is avoided.

\section{Experimental design}

Before evaluating the GSAs based on the experimentally acquired data, it is good to appropriately conceive the experiments themselves through an "experimental design" [328, 329, 352-354]. Accordingly, one has to identify the factors influencing the responses (features) of the SA in a screening process. The most relevant factors in gas sensing are the target analyte concentration and the influence of the main interferents (like humidity, concentration of the known gases in the sample, temperature and pressure). A good experimental design must provide a choice of factors to be considered and the number of experimental events (including replicates) needed for correct GSA characterization [352, 353]. Response surface methodologies are often employed. They consider that the response $x_{m, n}$ of a given sensor $n$, in the experimental event number $m$ (a certain gas exposure for instance), to the selected factors $\left\{f_{i}\right\}$ (gas concentrations for example) can be approximated through a polynomial of low order, containing linear and quadratic terms [323, 355]:

$x_{m, n} \cong \sum_{i=1, j=1, k_{i, j}=0}^{i=I, j=I, k_{i}+k_{j} \leq 2} c_{m, n, i, j} \cdot f_{m, i}^{k_{i}} \cdot f_{m, j}^{k_{j}}$

where $c_{m, i, j}$ are polynomial coefficients, $I$ the total number of considered factors, and $i, j, k_{i, j}, m, n$ indices (natural numbers). In Eq. (1) there are 1 free term, $I$ linear terms, $I$ quadratic onevariable terms, and $\left(\begin{array}{l}I \\ 2\end{array}\right)$ quadratic mixed (factor interaction) terms, leading to $G=2 I+1+\left(\begin{array}{l}I \\ 2\end{array}\right)$ coefficients (parameters). A full experiment having $M$ events (gas exposures) can be represented in the above approximation by an $M \times G$ design matrix $\boldsymbol{D}$ ( $M$ rows and $G$ columns). Its elements, $D_{m, n}$, are the monomials $f_{m, i}^{k_{i}} \cdot f_{m, j}^{k_{j}}$ contained in Eq. (1) for the factor values taken during event $m \in[1, \mathrm{M}]^{28}$. The index $n \in[1$, $N]$ of the column stands for combinations of the factor indices $i, j$ in any order (preserved along the design procedure). Inspection of the design matrix or significance tests enables the identification of the relevant factors. Current experimental design approaches include full and fractional factorial designs $[323,352]$. The full factorial design includes devised events

\footnotetext{
${ }^{28}$ In the design matrix, as in the data matrix, the rows express events (design events in this case), but the columns, in contrast, contain factor values (or products of factor values for the second-order terms) as they appear in the polynomial approximation given by Eq. (1).
} 
including all $I$ factors, each with different $L$ values (levels). Though more accurate, the full factorial design requires a huge number of experiments $\left(M=L^{I}\right)$. Therefore, a screening procedure at two relevant levels is commonly used in a preliminary step. The fractional factorial design discards, in a systematic manner, part of the events considered in the full factorial one, reducing the dimensionality of the design matrix. Although the design of the SAs with regard to the receptor materials, structure and number of devices has frequently been considered in the literature devoted to GGSs, there are very few attempts to design experiments for the evaluation of SAs. For example, in a paper dating back to 1995, Hierlemann et al. employed Box-Behnken and factorial design to reduce the calibration time of the Q-TSMR-SA and optimize the experiments [226].

\section{Sensor data evaluation}

The data collected during SA evaluation experiments require multivariate analysis because of their statistical nature, large number of factors being considered, and hidden correlations or structure [356, 357]. Dedicated multivariate methods provide the classification of the experimental events (cluster analysis [CA], discriminant analysis [DA], partial least squares discriminant analysis [PLS-DA]) [358-361], reduction of data dimensions without significant loss of information (principal component analysis [PCA] or factor analysis [FA]) [362-366] and multivariate calibration (multiple linear regression [MLR], principal component regression [PCR], partial least squares regression [PLSR]) [367, 368].

Unsupervised multivariate methods do not use a priori assumptions about the number, structure or identity of the data, unlike the supervised methods, which are based on previous information, typically gathered during training sets of experiments. This prior information can be the number and type of event classes.

\section{Unsupervised multivariate data analysis}

Cluster analysis [358] is a common unsupervised exploratory classification method. Events with similar patterns are included in the same homogeneous class/cluster. Because the patterns appear as points in the space of the features (the sensor outputs in the case of SAs), the clusters are spontaneously formed based on the distances between the events in this space. Generally, Euclidian metrics is employed to calculate the distances:

$$
d_{i, j}=\left[\sum_{n=1}^{q}\left(x_{i, n}-x_{j, n}\right)^{2}\right]^{\frac{1}{2}}
$$

This supposes orthogonal, linearly independent coordinates, which is mainly not the case, the responses of the sensors being often correlated. Moreover, starting with four features, it is not possible to graphically visualize the clustering process. A frequent solution is the projection of data on orthogonal coordinate axes built up as a linear combination of the dependent variables in the feature space. Further simplification of the procedure may be achieved by using the PCA (see next paragraph and the examples in Table 1). Alternatively, the Mahalanobis distance may be employed [369-371]. Once the problem of correct calculation of distances is worked out, the classes can be hierarchically constructed, starting with one-element clusters and merging, step by step, the closest ones. The plot of the distances between clusters appears as a dendrogram (the plot resembles the branches of a tree), as displayed in Fig. 16. Alongside crisp/ hard clusters habitually reported in the literature, fuzzy clustering has also been considered [372]. In this case, the events belong to different classes with different degrees of membership.

Principal component analysis (PCA) is the most widely used unsupervised exploratory multivariate method for evaluating the SA output $[323,362-365]$. PCA replaces the $N$ correlated coordinates in the feature space with a set of $A \leq$ $N$ linear combinations of them, which are orthogonal to each other, and hierarchically ordered according to the amount of variability transferred from the original data. Named by Hotelling [373], principal components (PCs) define new abstract features, generally with no experimental meaning.

The event producing the original score vector $\boldsymbol{x}_{m}$ will also produce an abstract score vector (single row matrix) $\boldsymbol{t}_{m}=\left(t_{m, 1}\right.$, $\left.t_{m, 2}, \ldots t_{m, \alpha}, . . t_{m, A}\right)$ with dimensionality $A$ in the new feature space. Formally, each element $t_{m, \alpha}$ of the vector $\boldsymbol{t}_{m}$ can be expressed as a linear combination of the elements $x_{m, n}$ of $\boldsymbol{x}_{m}$ : $t_{m, \alpha}=\sum_{n=1}^{N} w_{m, \alpha, n} \cdot x_{m, n}$

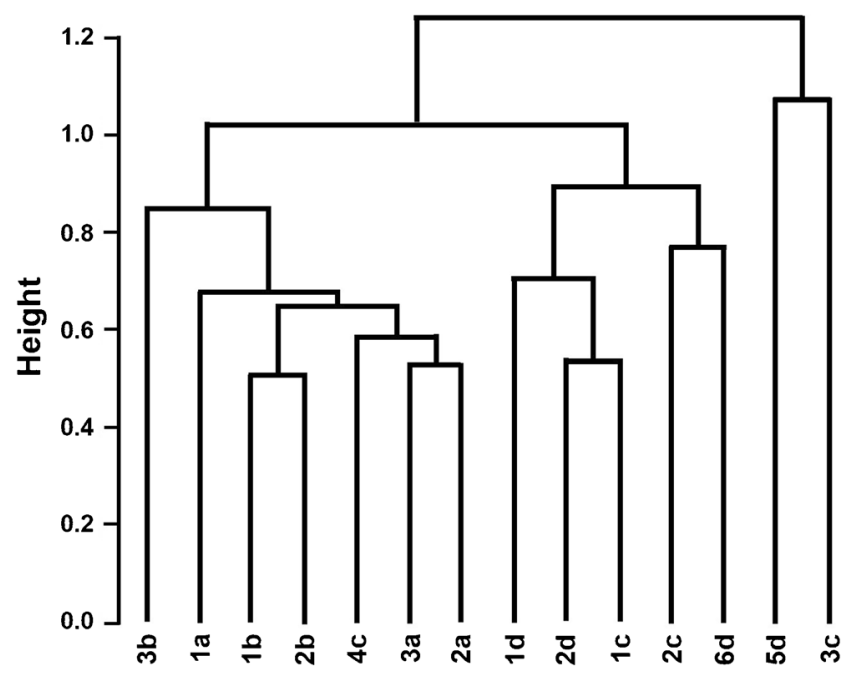

Fig. 16 Hierarchical cluster analysis. Dendrogram obtained with 14 fluoroalkyloxy-substituted phthalocyanines. Reproduced with the kind permission of Elsevier B.V. from reference [107] 
where $w_{m, \alpha, n}$ are the combination coefficients (weights of the original features) and $\alpha \in[1, \mathrm{~A}]$ is the index of the $P C \mathrm{~s}$. In principle, one has to seek all coefficients $w_{m, \alpha, n}$ so that all PCs are orthogonal and $P C 1$ carries the largest variability, $P C 2$ the next largest, and so on (see Fig. 17a). It is worth noting that an arbitrary increase of all $w_{m, \alpha, n}$ will result in an arbitrary increase of all $t_{m, \alpha}$, leading to the failure of the procedure. Therefore, it is necessary to additionally request the normalization of the coefficient set $\left\{w_{m, \alpha}\right.$, $\left.{ }_{n}\right\}$ for fixed $m \& \alpha$. In many cases, the first few $P C$ s are enough to bear almost all information contained by $\boldsymbol{X}$, so that the matrix $\widehat{\boldsymbol{X}}$ (the hut is a common notation for estimated values), estimated with the help of these $P C$ s, differs from the original $\boldsymbol{X}$ by some small amounts only, the residuals, stored in the matrix of residuals $\boldsymbol{E}=\boldsymbol{X}-\widehat{\boldsymbol{X}}$. In this way, PCA ensures a practical reduction of the dimensionality of the feature space. Furthermore, $\boldsymbol{X}$ can now be factorized using the values of the new features for the given events (the new "scores") and the "loadings" (components) of the PCs in the old feature frame:

$\boldsymbol{X}=\boldsymbol{T} \boldsymbol{P}^{T}+\boldsymbol{E}=\widehat{\boldsymbol{X}}+\boldsymbol{E}$

The matrix $\boldsymbol{T}$ of scores (actually new sores) is $M \times A$-dimensional, and that of loadings, $\boldsymbol{P}, N \times A$-dimensional, so that its transpose $\boldsymbol{T}^{T}$ is $A \times N$-dimensional. PCA, in the limit of selected $P C \mathrm{~s}$, explains a fraction $\left[\|\boldsymbol{X}\|^{2}-\|\boldsymbol{E}\|^{2}\right] /\|\boldsymbol{X}\|^{2}=$ $\|\widehat{\boldsymbol{X}}\|^{2} /\|\boldsymbol{X}\|^{2}$ of the variability contained by $\boldsymbol{X}$ [365]. The PCA estimations are mainly visualized as scatter plots of scores and loadings either separated or together in a common biplot. In the case of biplots, the graphical separation of the events might not be the real one due to the different units or scaling factors being used (commonly, the loadings are scaled to 1 while the scores bear the variability of the corresponding PC). There are several available approaches to find the PCs, including nonlinear iterative partial least squares (NIPALS) [362, 374], singular value decomposition (SVD) [349] and covariance matrix. The authors reporting on GGSs mainly use PCA to check event clustering and present their results as score plots. More than half of the papers listed in Table 1 include PCA approaches. A graphical example is reproduced in Fig. 17b.

Factor analysis (FA), despite its formal resemblance to PCA, adopts a different point of view [366, 375-377]. Instead of seeking a linear combination of dependent variables to obtain new ones (the $P C \mathrm{~s}$ ), orthogonal and ordered with respect to the carried variability (as PCA does), FA looks for a set of $m$ independent (random) variables (common factors$f_{m}$ ) whose linear combinations render the dependent variables up to a residual variable (specific factor) ${ }^{29} e$ :

$$
x_{n}=\sum_{m}^{M} a_{n m} \xi_{m}+e_{n} \quad n \in[1, N]
$$

\footnotetext{
${ }^{29}$ This term might also contain the errors.
}

The coefficients $a_{n m}$ are the factor loadings of the variable $x_{n}$ on the $m^{\text {th }}$ factor. By using the name "common factor", one intends to point out the influence of such independent variable on all dependent variables. Equation (5) is apparently identical to the linear part of Eq. (1), but apparently only because the factors $f_{i}$ in Eq. (1) are real factors, with physicochemical relevance, while the $f_{m}$ factors of FA lack this character, being formal entities. In matrix form, Eq. (5) becomes $\boldsymbol{x}=\boldsymbol{A} F+\boldsymbol{e}$ and shows that FA is a model (regression - see next paragraph)-based approach, while PCA is not (in fact, not necessarily).

\section{Supervised multivariate data analysis}

Discriminant analysis (DA) [359] attempts to assign events (objects) whose features are categorical variables to predefined classes. The factors considered by DA are continuous variables. The method seeks discriminant functions of independent variables simultaneously, leading to the largest separation between classes and the smallest ones inside each class. Commonly used approaches include linear discriminant functions (LDA, initially introduced by Fisher for two classes only $[378]^{30}$ ), soft independent modelling of class analogy (SIMCA), [379, 380] and $k$-means clustering [381]. Nice LDA discrimination of methanol, ethanol, acetone and tetrahydrofuran with a very simple paper cantilever SA was reported by Fraiwan et al. [240]. By using quadratic DA (QDA) and a virtual Q-TSMR-SA, Speller et al. obtained good separation of a large number of VOCs [236].

\section{Univariate and multivariate calibration}

Calibration entails establishing a connection between two sets (blocks) of variables. For SA data, they are the sensor responses $\left\{x_{m, n}\right\}=\boldsymbol{X}$, one for each sensor $n$ in the measurement number $m$, and the factors $\left\{f_{m, i}\right\}=\boldsymbol{F}$, mainly the concentrations $\left\{c_{m}, i\right\}=\boldsymbol{C}$ of different $I$ gases in the test mixture employed during measurement $m$. The number $M$ of calibration tests must exceed the number $N$ of the sensors in the SA in order to allow a nontrivial solution for the approach $(M \geq N)$.

Univariate calibration relates one feature (sensor response) to one factor (target gas concentration). However, it does not involve only one event. To reduce the effect of experimental errors affecting both feature and factor, and to identify the type of relationship between them, a series of measurements (with replicates) is performed. In this way, one obtains a column vector of features $\boldsymbol{x}$, containing the responses of the sensor to the corresponding concentrations of the analyte (also

\footnotetext{
${ }^{30}$ The article was cited exclusively for multivariate data analysis purposes. The authors delimit themselves from the legally and morally inadequate content of the article and publishing journal.
} 


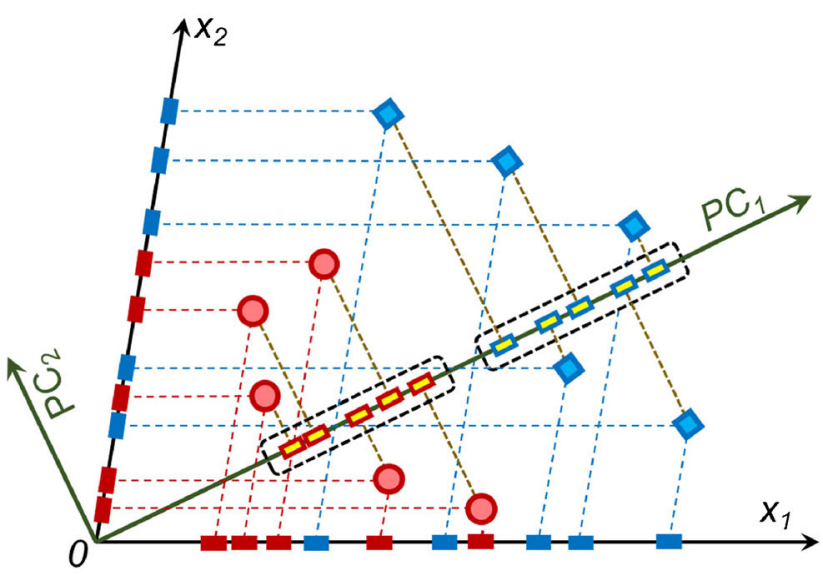

a

Fig. 17 (a) Two classes of events in a bidimensional feature space, which appear as mixed on both linearly dependent axes $\mathrm{x}_{1}$ and $\mathrm{x}_{2}$, are discriminated on the principal components. Moreover, the whole variability is carried by $P C_{1}$. The example could express the responses

written as a column vector). In many cases, the relationship sought appears to be a linear regression $\boldsymbol{x}=s \cdot \boldsymbol{c}+\boldsymbol{e}_{x}$ which, through simple matrix algebra, results in [323]:

$s \cong\left(\sum_{m=1}^{M} c_{m} \cdot x_{m}\right) /\left(\sum_{m=1}^{M}\left(x_{m}\right)^{2}\right)$

Here, the scalar $s$ is the sensor sensitivity and $\boldsymbol{e}_{x}$ the errorvector of responses. The index 1 of the first rank column matrices (expressing only one feature) was omitted. Because the task of the sensors is to provide the value of the factor (concentration) when the feature (response) is known, one needs to consider an inverse calibration $\boldsymbol{c}=b \cdot \boldsymbol{x}+\boldsymbol{e}_{c}$, where $\boldsymbol{e}_{c}$ is the error-vector of concentrations. In the limit of experimental errors, $b$ is the inverse of the sensitivity, and its estimation is given by:

$b \cong\left(\sum_{m=1}^{M} c_{m} \cdot x_{m}\right) /\left(\sum_{m=1}^{M}\left(x_{m}\right)^{2}\right)$

If the sensor has a nonzero baseline (the sensor response in the absence of the analyte is not zero, but $\boldsymbol{x}_{\mathbf{0}}$ ), then one has to subtract the baseline vector $x_{0}$ from the vector of the responses $\boldsymbol{x}$ in the calibration equation, so that $\boldsymbol{c}=b \cdot\left(\boldsymbol{x}-\boldsymbol{x}_{0}\right)+\boldsymbol{e}_{c}$. The vector $\boldsymbol{x}_{0}$ contains the actual values of the baseline during the experiments $m \in[1, M]$.

Multivariate calibration broadens the univariate calibration towards the case of multiple factors and features (several analytes and several sensors, possible in a SA)

Multiple linear regression (MLR) is a generalization of the univariate linear regression and is expressed by the matrix extension of the corresponding univariate equations ${ }^{31}$ :

\footnotetext{
$\overline{31}$ The algebraic trick of multiplication with the transpose matrix in the following equation is required because $\boldsymbol{X}$ is generally not a square matrix and therefore is not invertible.
}

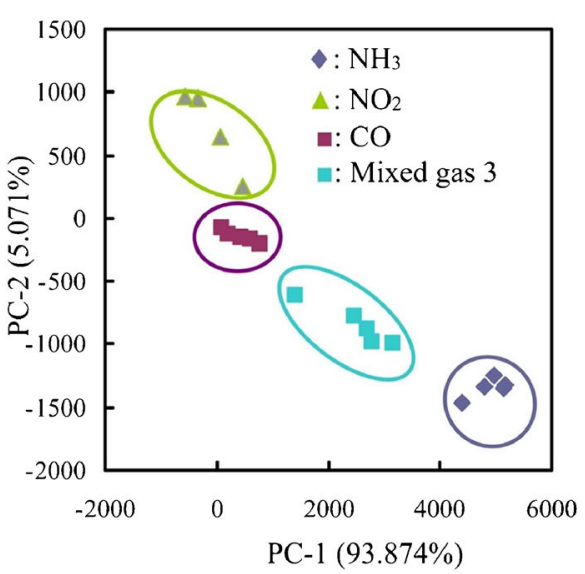

b

of two gas sensors which have a certain degree of similarity. (b) Example of PCA score plot displaying analyte clustering. Panel (b) was reproduced with the kind permission of Elsevier B.V. from reference [251]

$\boldsymbol{C}=\boldsymbol{X} \cdot \boldsymbol{B}+\boldsymbol{E}_{\boldsymbol{C}} \rightarrow \widehat{\boldsymbol{B}}=\left(\boldsymbol{X}^{T} \boldsymbol{X}\right)^{-1} \boldsymbol{X}^{T} \boldsymbol{C}$. With the matrix $\widehat{\boldsymbol{B}}$ obtained in the calibration procedure, one can predict (estimate) the unknown concentrations for a new sample containing the same analytes: $\widehat{\boldsymbol{C}}_{\text {new }}=\boldsymbol{X}_{\text {new }} \cdot \widehat{\boldsymbol{B}}$. The method has a non-negligible disadvantage. It requires knowledge of the responses and concentrations for all relevant compounds in the training sample.

Principal component regression (PCR) was developed to directly relate the factors (concentrations of the analytes) to the scores on the first PCs, through an abstract matrix $\boldsymbol{R}$, avoiding the large amount of data and computations demanded by MLR. Thus:

$$
\begin{aligned}
\boldsymbol{C} & =\boldsymbol{T} \cdot \boldsymbol{R}+\boldsymbol{E}_{\boldsymbol{C}} \rightarrow \widehat{\boldsymbol{R}}=\left(\boldsymbol{X}^{T} \boldsymbol{X}\right)^{-1} \boldsymbol{X}^{T} \boldsymbol{C} \quad \& \quad \widehat{\boldsymbol{C}}_{\text {new }} \\
& =\boldsymbol{X}_{\text {new }} \cdot \widehat{\boldsymbol{R}} .
\end{aligned}
$$

(The index "new" addresses the values in a new experiment, either validation or prediction).

Partial least squares regression (PLSR) ${ }^{32}[346,367,382$, 383] uses two matrix decompositions (often referred to as models), one for the features, as in PCA, and another for the factors (concentrations in the gas sensor case):

$\boldsymbol{X}=\boldsymbol{T} \boldsymbol{P}^{T}+\boldsymbol{E}_{\boldsymbol{X}} \quad \& \quad \boldsymbol{C}=\boldsymbol{U} \boldsymbol{Q}^{T}+\boldsymbol{E}_{\boldsymbol{C}}$

where $\boldsymbol{U}$ and $\boldsymbol{Q}$ play roles analogous to scores and loadings, respectively, for the data block $\boldsymbol{C}$. The presence of the matrices of residuals for features and factors in Eq. (9) indicates that a reduction of dimensionality is performed, so that some unexplained variability remained. If the sensor responses and

\footnotetext{
32 The PLSR variant presented here is known as PLSR II and allows one to tackle all factors $\boldsymbol{C}$ at once. An older version, PLSR I, introduces the factors one by one as column vectors $c_{\alpha}$ (one element for each event).
} 
concentrations are directly proportional, then one would expect a linear relation $\boldsymbol{u}_{i}=\boldsymbol{b}_{i} \boldsymbol{t}_{i}$ between the elements of $\boldsymbol{U}$ and $\boldsymbol{T}$. $\boldsymbol{u}_{i}$ and $\boldsymbol{t}_{i}$ are column vectors of $\boldsymbol{U}$ and $\boldsymbol{T}$, while $\boldsymbol{b}_{i}$ is the equivalent of the regression coefficients in MLR or PCR [323, 367]. The best prediction $\widehat{\boldsymbol{C}}$ is obtained when the matrix of the residuals $\boldsymbol{E}_{\boldsymbol{C}}=\boldsymbol{C}-\boldsymbol{U} \boldsymbol{Q}^{T}=\boldsymbol{C}-\widehat{\boldsymbol{C}}$ reaches its minimum for the given events. PLSR is well suited for overdetermined data sets (more events than features). PLSR is rather widespread in the GGS community. For instance, the application of PLSR to the shelf life of eggs gives good results, as Fig. 18 shows.

Partial least squares discriminant analysis (PLS-DA) is an algorithm (certain authors consider it a method) used to perform supervised classification adapting PLSR to clustering problems [360, 361, 386].

\section{Artificial intelligence in chemometrics}

Artificial intelligence (AI) has emerged from the increasing capability of computers to perform complex tasks, some of them already listed/foreseen in the birth document of the concept [387]. "A historical survey of algorithms and hardware architectures for neural-inspired and neuromorphic computing applications" was published not too long ago [388]. The impact of AI on chemometrics is constantly growing [325, 389, 390], with the most widely employed being the artificial neural networks (ANN) [391-397], support vector machines (SVM) [398-404], genetic algorithms (GA) [405, 406] and expert systems (ES) [404, 407-409]. The use of AI in conjunction with GGSs is summarized in Table 2.

Artificial neural networks (ANN) are computing algorithms loosely mimicking the human brain and neural system. The origins of ANN are related to the relevant papers of McCulloch and Pitts and of Hebb, which provide a logical/ mathematical understanding of the nervous [415] and sensorial [416] activity, respectively bridges "the gap between neurophysiology and psychology", addressing synaptic plasticity and associative learning [417]. The building block of the ANN is the artificial neuron (AN) as a conceptual counterpart of its biological model (for the anatomy and physiology of biological neurons and neural systems, please refer to the literature $[418,419])$. The first AN implementation (the logic threshold unit [LTU]) by McCulloch and Pitts has several Boolean inputs and a single digit output, which is 1 if an internally threshold is exceeded by the sum of all inputs and 0 otherwise. The "perceptron" ${ }^{\text {"3 }}$ proposed by Rosenblatt [420] and refined by Minsky and Papert [421] increased the functional capability of LTU. It allows for weighted inputs, expressing different degrees of importance, but retains the same two-level output triggered by a discrete decision function. The utility of separated neurons is reduced, and their applicative power comes out from interconnections in ANN [388, 391, 396,

\footnotetext{
33 The term "perceptron" is used either to define a certain type of AN, or to address a class of simple ANNs containing perceptron-ANs in its nodes.
}

422]. The network approach requires new or improved/adapted features of ANs. Figure 19a illustrates a widely used AN structure.

ANNs are built following two main architectures: feedforward networks (FFN) and recurrent networks (RN). FFNs (see Fig. 19b) are constructed from two or more layers of ANs. The input layer is supplied with the data to be processed (a vector $\boldsymbol{x}_{m}$ expressing the event $m$ in the $N$-dimensional feature space of a $\mathrm{SA}$, for example). The output of each AN from one layer is sent to all inputs of the ANs in the next layer up to the output layer, which delivers an output variable $\boldsymbol{y}_{m}$ (here symbolized as a vector, which could indicate the class to which the event $m$ belongs, if a classification problem is to be solved by the ANN). To fit the ANN to its task, an adaptive control is realized, usually by tuning the weights at each node. It has been shown that the functionality of ANNs can be increased significantly when recurrent architecture is chosen, that is, feedback loops are considered [422-425]. The most commonly employed feedback type is the "back-propagation" (BP). It involves two operating steps. In the first step the ANN accomplishes its task in a feed-forward mode, with all weights fixed. Afterwards, it estimates the error it made, providing error parameter(s). In the next step the weights are actualized to correct/minimize the error. An unsuitable choice of starting weights and of their tuning procedure might result in unstable ANN operation and eventually oscillating/divergent output (this is a common issue in the systems controlled by feedback) [396, 426]. The ANNs are able to learn, that is, to adapt their free parameters in such a way as to be able to perform certain tasks [427]. In supervised learning, a training set of known events is run by the ANN, which receives for each input vector the true output value(s). Based on these data, the algorithm adapts itself to provide maximal accuracy during a given number of training cycles. If it fails, it must be improved and checked again. In the case of unsupervised learning, no "true" output data are available, so the training is successful when producing consistent output, like good classification patterns. Zupan and Gasteiger proposed a short and intuitive approach to this topic [392]. An exhaustive overview on "deep learning in neural networks" up to 2015 was provided by Schmidhuber [428]. Very frequently researchers in the field of GGSs use standard chemometric methods, mainly PCA, together with ANN (see Table 2).

Support vector machines (SVM) are supervised algorithms employed for non-probabilistic classification and regressions. SVM started from linear binary classification [398] (analogous to LDA) and evolved towards elaborate nonlinear algorithms. Initially a "training algorithm for optimal margin classifiers" [429] was conceived, whose task was to find the hyperplane in the feature space that best discriminated two classes of events ${ }^{34}$

\footnotetext{
${ }^{34}$ In the classification methods, the feature space of the data set becomes an input space, while the output space is categorical. For SVM, the term "feature space" is therefore employed for a higher-dimensional space obtained by the mapping of the input space (see later in the text). Thus, the SVM feature space is in fact a space of features of features.
} 


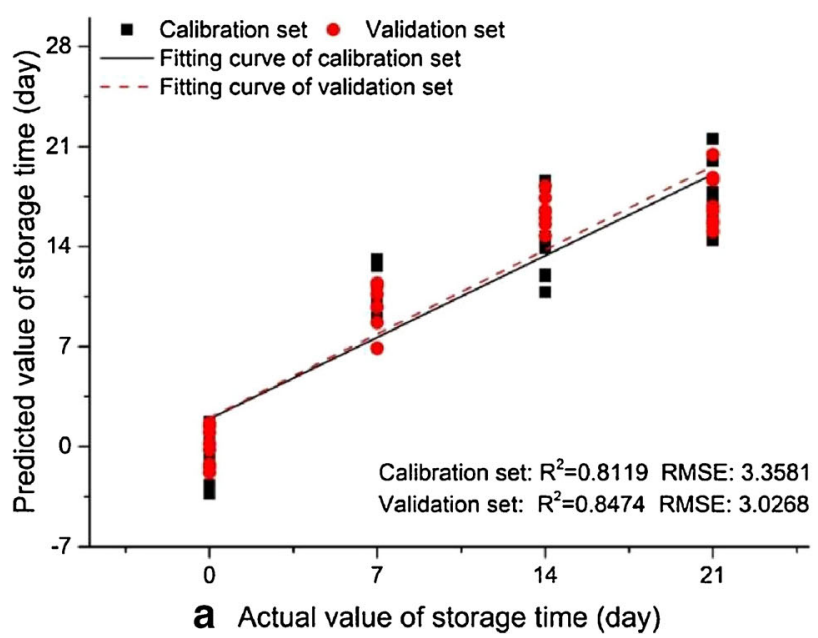

Fig. 18 PLSR prediction of the on-shelf life of eggs. (a) PLSR results for original data, (b) PLSR results after an intermediate KPCA processing. KPCA (Consider references [384] and [385] for additional information on

(see Fig. 20a). Mathematically, this means that the sum of distances from this hyperplane to the closest event(s) of each class - the margin - has to be maximized [399, 430]. The vectors in the feature space [431] that specify the position of the margin events - the "support vectors"-play a special role in the approach, determining the location of the discriminant hyperplane $[399,430]$. The "hard margin" addressed above can seldom be obtained because of the experimental noise or incomplete variability explanation. Therefore, Cortes and Vapnik relaxed the separation condition at the expense of classification accuracy, building up a soft margin classifier [432]. In this case, the set of the support vectors is completed by additional, non-margin, ones. The maximum margin hyperplane approach can also be extended for nonlinear classifiers, as emerged from the paper by Boser et al. [429]. First, one should note that the margin optimization problem for both hard and soft cases involves the event vectors as direct products $\left\langle\boldsymbol{x}_{i}, \boldsymbol{x}_{j}\right\rangle$ only. Therefore, if one maps the event vectors from the $N$-dimensional feature space into a higherdimensional space (see footnote 34$) x \rightarrow^{\varphi} \varphi(x)$ the optimization problem will "move" into the new space, but will still be based on the direct products of the type $\left\langle\boldsymbol{\varphi}\left(\boldsymbol{x}_{i}\right), \boldsymbol{\varphi}\left(\boldsymbol{x}_{j}\right)\right\rangle$ only. Provided the function $\varphi$ is suitably chosen, a linear discriminant might be found in the new space. However, instead of looking for convenient mapping functions $\varphi$, it is much easier to seek "kernel functions" $k$ of two event variables that straightforwardly map the pair $\left(\boldsymbol{x}_{i}, \boldsymbol{x}_{j}\right)$ to direct products $\left\langle\boldsymbol{\varphi}\left(\boldsymbol{x}_{i}\right), \boldsymbol{\varphi}\left(\boldsymbol{x}_{j}\right)\right\rangle$. It remains now to replace the direct products $\left\langle\varphi\left(\boldsymbol{x}_{i}\right), \varphi\left(\boldsymbol{x}_{j}\right)\right\rangle$ in the optimization problem with the corresponding kernel function $k\left(\boldsymbol{x}_{i}, \boldsymbol{x}_{j}\right)$ and to train the algorithm. Detailed explanations and examples are given by Burges [399] and Luts et al. [403].

Looking to Fig. 20a, one could think that the vector $\boldsymbol{w}$ indicating the direction of the discriminating plane $\Delta$ would be the same as that of $P C_{1}$ in a PCA approach to the data set. That is usually not the case, because $P C_{1}$ is chosen to maximize the explained variability of the whole data set, while $\boldsymbol{w}$ is

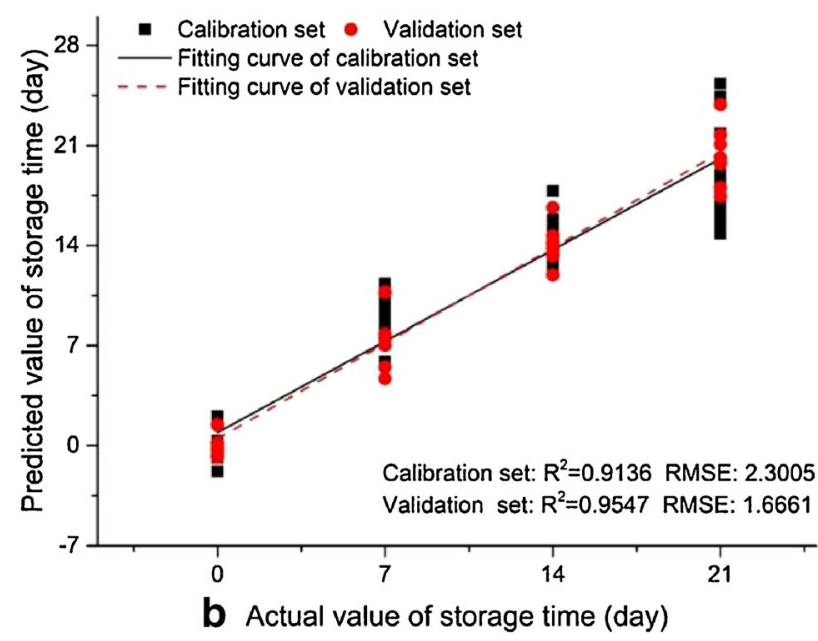

KPCA. See also the section dedicated to support vector machines below.) stands for kernel PCA. Reproduced with the kind permission of Elsevier B.V. from reference [343]

used to maximize the margin, which depends on only a few data points, pointed to by the support vectors. Generally, it worth noting that all AI methods involve adaptive and autonomous algorithms, while "traditional" multivariate data analysis is based on stated numerical procedures.

An example of combining kernel functions with PCA towards KPCA and further use of PLSR is given in Fig. 18 above.

The genetic algorithms and expert systems are not popular in the field of GGS.

A general approach to chemometric and AI topics is given in the Handbook of Machine Olfaction [433].

\section{Unconventional theoretical and numerical approaches to sensor data analysis}

Unconventional theoretical and numerical approaches to sensor data analysis are used by the researchers in their attempt to better understand, simulate and fit the experimental information. Davide et al. propose a blockstructured mathematical model for the GGS based on TSMRs [434, 435]. From the adsorption isotherms of formaldehyde on bio-inspired poly(dopamine) Yan et al. derive the standard enthalpy of sorption which well correlates with the DFT simulations [314]. Sharma et al. used a relatively simple approach, based on the time dependence of the sensor response, to obtain analyte recognition [436]. Accordingly, the maxima of the sensor responses for an exposure to analyte concentration pulses (named "dynamic headspace technique" by the authors) are shifted in time, somehow as by a chromatographic column, improving the recognition procedure. This provided a better way to evaluate the amount of linalool, which is a relevant VOC for traditional tea flavor. Regmi et al. observed that the ratio of the change in 


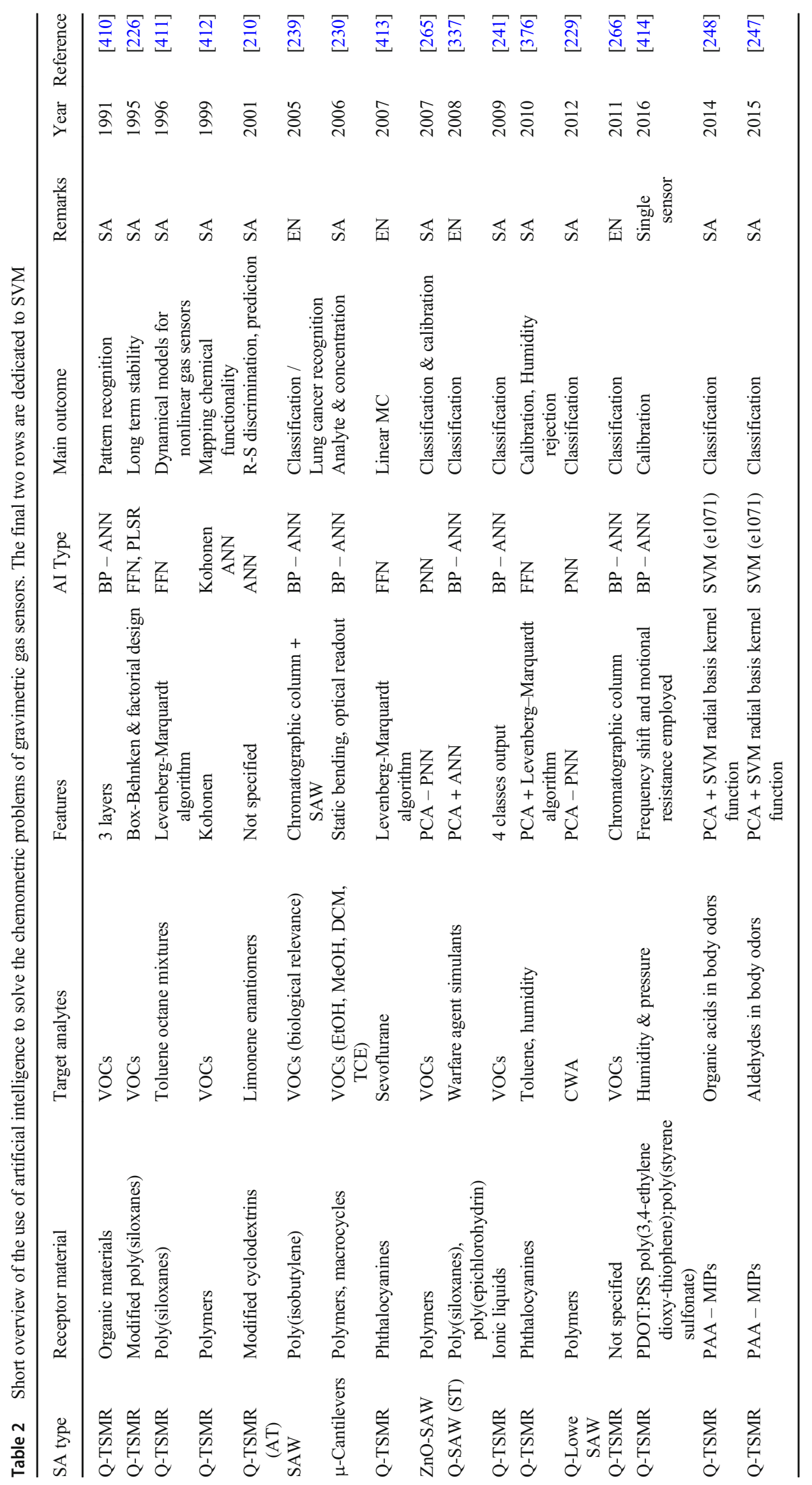




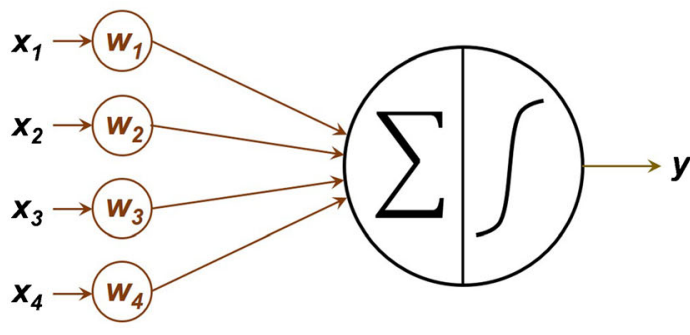

a

Fig. 19 (a) The conventional sketch of an artificial neuron. The inputs $\left\{x_{n}\right\}$ (vector $\boldsymbol{x}$ ) are adjusted with the weights $\left\{w_{n}\right\}$ (vector $\boldsymbol{w}$ ) and added with the block $\Sigma$. The result is squashed with the transfer function $\int$ and

resonance frequency to the change in motional resistance (the resistance in the equivalent circuit of the TSMRs which corresponds to the mechanical damping of the crystal) was concentration-independent but proportional to the molecular weight of the absorbed analyte [178]. This proportionality allowed the identification of the VOCs for which the device was sensitive according to their mass. The procedure was upgraded through the use of virtual sensor arrays [237] (see Fig. 20b). A molecular dynamics approach was successfully used by Khanniche et al. to fit

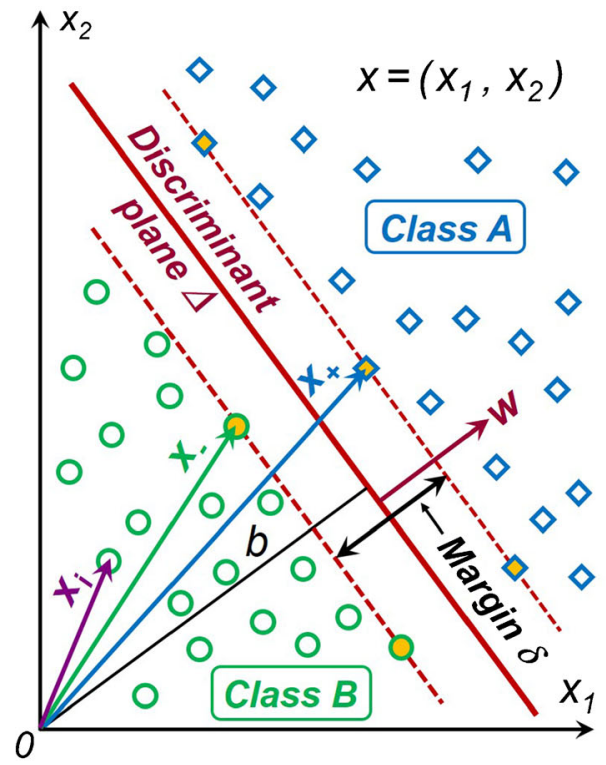

a

Fig. 20 (a) Hard-margin SVM: two-dimensional sketch. The events are displayed as $\boldsymbol{x}$-vectors with two components $x_{1}$ and $x_{2}$, and are classified as class A (green open circles) and B (blue open rhombs), respectively. The classes are disjoint and best separated by the plane $\Delta$. The margin events (orange filled symbols) are specified by the support vectors (from which only $\boldsymbol{x}_{+}$and $\boldsymbol{x}_{-}$are depicted in blue and green, respectively). The vector $\boldsymbol{w}$ (perpendicular to $\Delta$ ) mathematically describes the discriminant

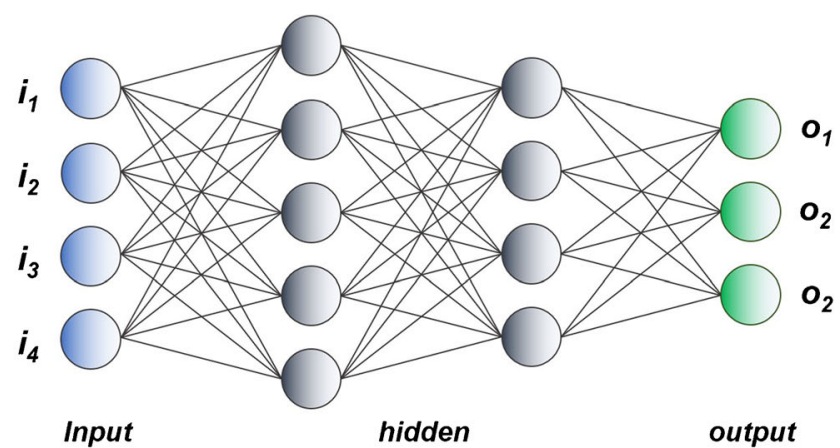

b

delivered as output $y$ (a scalar in the sketch). (b) Simple feed-forward ANN with four layers of which two are hidden. The data flow from left to right in the sketch

the output of a TSMR covered with methylated mesoporous silica in interaction with vapors of the nitroaromatic compounds 2,4-dinitrotoluene (2,4-DNT) and trinitrotoluene (TNT) [437]. A humidity correction was performed so that the simulations agreed with the experimental data. Based on the information from the gravimetric responses of TSMRs coated with calixarene films and colorimetric analysis, Kostyukevych et al. proposed a nanostructure model of the material in which almost spherical stochastically distributed nanocavities enabled the gas (ethanol)

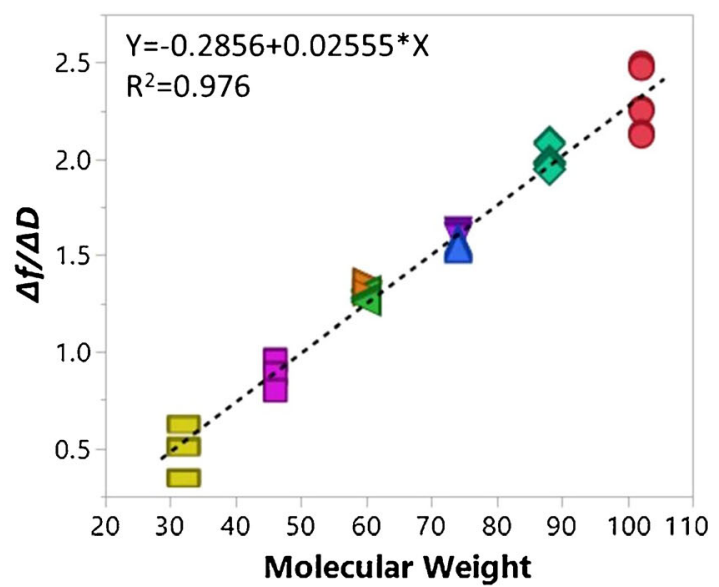

Sample

$=$ methanol $\quad$ ethanol
2-propanol $\Delta$ 1-butanol
3-methyl-1-butanol

b

plane direction which lies at the distance $b$ from the origin. The margin $\delta$ is measured along $\boldsymbol{w} \cdot \boldsymbol{x}_{i}$ is a generic event, as used in SVM formalism. (b) Analyte discrimination through the proportionality of the TSMR output parameter ratio (frequency shift over dissipation factor) and the molar mass. Panel (b) reproduced with the kind permission of Elsevier B.V. from reference [237] 
adsorption [99]. Nimsuk and Nakamoto proved the utility of an original and unexpected approach to the classification of apple and muscat flavors with variable concentrations [438]. They applied the short-time Fourier transform to the SA data, which indicated different time constants of the sensors for different flavors. In the final step, a linear vector quantization (LVQ) ANN was used for successful discrimination of the two flavors. Iglesias et al. used blank Si micro-cantilevers to discriminate and quantify $\mathrm{H}_{2}, \mathrm{CO}, \mathrm{CO}_{2}, \mathrm{CH}_{4}, \mathrm{He}$ and $\mathrm{O}_{2}$ diluted in nitrogen in percent ranges [439]. The physical sensing principle is based on the differences in the density and viscosity of these gases, which modify the resonant frequency and quality factor of the device upon environment composition. In the space of the relative density and viscosity (referred to nitrogen), each analyte appears on a certain straight line, whose angle with the carrier gas $\left(\mathrm{N}_{2}\right)$ line indicates the analyte type, while the distance from the origin is proportional to the concentration (see Fig. 21).

\section{Targeted analytes and application}

The spectrum of GGS applications reported in the literature is very large and overlaps that of other sensor types, even that of established ones such as the capacitive for humidity or chemoresistive for reducing gases. A full characterization of the sensors/SAs is not always performed or provided in the papers, probably because of the effort and time expense involved. The authors usually indicate the target analytes for their sensors and warn of possible interference, contamination or adverse action from other gases/vapors. In the case of critical requirements, researchers also prove the suitability of their devices for the given task. The review includes two paragraphs below dedicated to GGS and SA applications, one addressing targeted analytes and the other the expected sensor use. Subsequent rough classifications are also given. Almost all papers addressed in this section are listed in the Annex table.

\section{Targeted analytes}

\section{Relevance of gaseous analytes detected with GGSs and legal/institutional regulations concerning their concentration limits}

There are several kinds of analytes appropriate for detection with GGSs. Some have injurious biological effects (are toxic, irritants, carcinogenic, mutagenic, reprotoxic) [440], while others are flammable or explosive [441]. There are many others which are chemically inoffensive but their concentration in the atmosphere or in closed spaces is relevant for industry, agriculture and other human activities. The physiological and clinical consequences of harmful gases/vapors, starting with cutaneous contact and inhalation, and following the whole metabolic process, are the subject of numerous articles [442, 443] and textbooks [440, 444] (also for veterinary use [445]). Efforts are spent in identifying hazards as sources of potential damage/harm to individuals and optimizing their assessment [446-449]. The exposure of persons, animals and the environment to harmful gases/vapors is legally regulated worldwide. Accordingly, several concentration limits are established for noxious agents which, according to medical investigations, would be still acceptable without adverse effects. They differ, more or less, from country to country, and are included in the documentation of the authorized national, European and international institutions such as the Occupational Safety and Health Administration (OSHA) and National Institute for Occupational Safety and Health (NIOSH) in the United States (USA), the European Chemicals Agency (ECHA) of the European Union (EU) through its Registration, Evaluation, Authorisation and Restriction of Chemicals (REACH) regulation, the German Institute for Work Safety (Institut für Arbeitsschutz [IFA]), British Health and Safety Laboratory (HSL), Japanese National Institute of Occupational Safety and Health (JNIOSH), Chinese Standard Committee of Public Health (CSCPH) and Council for Occupational Health and Safety of South Africa (SACOH). Through the implementation of the Globally Harmonized System of Classification and Labelling of Chemicals (GHS), an international agreement managed by the United Nations, the national/regional safety norms should converge towards joint regulations, simplifying sensor evaluation and certification procedures [450-452]. In the case of gases, both the exposure dose and maximal concentration are relevant for the evaluation of professional hazards. At present, the US standards are the most commonly used in the literature. The permissible exposure limit (PEL) is the legal exposure limit for employees in the USA. PEL values are given by the Code of Federal Regulations (CFR), Part 1910-Occupational Safety and Health Standards, Section 1910.1000, Air contaminants. The threshold limit value (TLV) of a toxic substance is the concentration at which a person can be exposed at work every day for whole life. TLV is a reserved term of the American Conference of Governmental Industrial Hygienists (ACGIH) and has no regulatory nature. It can be evaluated for 8-hour working days, 5 working days a week, as the threshold limit value-time-weighted average (TLV-TWA). The short-term exposure limit (TLVSTEL) indicates the concentration limit for 15 minutes of exposure, while the ceiling limit (TLV-C) is the upper concentration for any exposure which still does not affect the health. The recommended exposure limit (REL) and immediately dangerous to life and health (IDLH) are reserved terms from NIOSH as guidelines of this institution. In the framework of EU-REACH, two concentration limits have been defined: the derived no-effect level (DNEL) is the "exposure level above which humans should not be exposed", and the predicted no-effect concentration (PNEC) is the concentration for which "no adverse effects" are expected. The Arbeitsplatzgrenzwert (AWG) is the German 
Fig. 21 Physical detection and discrimination of gases with uncoated micro-cantilevers. Reproduced with the kind permission of Elsevier B.V. from reference [439]

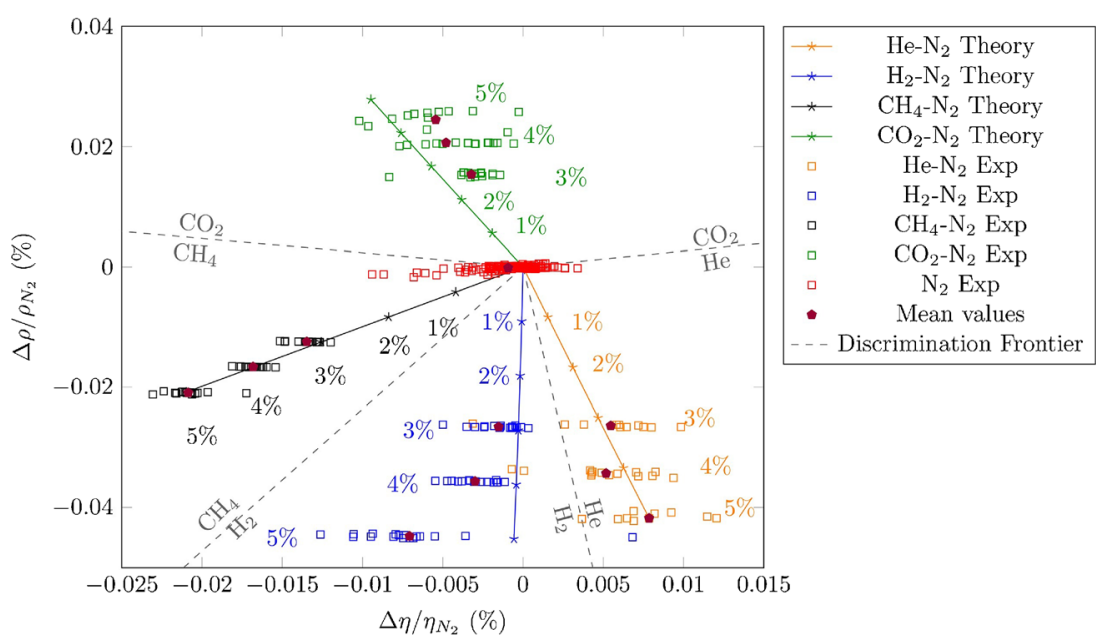

(IFA) equivalent of PEL. It replaces the older Maximale Arbeitsplatz Konzentration (MAK). International occupational exposure limits and many safety details can be retrieved from the large open-access database of the "Information system on hazardous substances of the German social accident insurance" GESTIS (Gefahrstoffinformationssystem) [453, 454]. As obvious from the tables associated with the norms, the values of the different limits are steadily decreasing. This fact reflects, on one hand, the increase in the known harmful consequences of diverse chemicals (thanks to progress in biology and medicine), and on the other hand a more restrictive definition of a healthy organism. Table 3 includes some example of concentration limits for the gases/vapor frequently addressed by researchers in the field of GGSs.

Highly toxic substances, some possibly produced as chemical warfare agents (CWA) [455], are the subject of a different jurisdiction. The harmful effect of these substances is measured by the lethal dose/concentration (LD100/LC100) or median lethal dose/concentration (LD50/LC50), which gives the amount of the agent that would cause death with an indicated probability of $100 \%$ or $50 \%$ [456, 457]. The required toxicologic experiments are performed on test animals under legally/ ethically acceptable conditions and extrapolated to humans. For safety reasons, the very toxic gases and vapors are replaced in gas-sensing tests with simulants, which are chemically very close to the substituted compounds but with no/low toxicity [456]. Lavoie et al. have given useful information on simulant types and their similarity to a large number of CWAs, suggesting chemoinformatic methods to detect them [458]. Additional information about toxic substances can be found on the NIOSH webpage or in EC No. 1272/2008 "Guidance on the Application of the CPL Criteria". Table 4 includes a few CWAs and the simulants commonly investigated for gravimetric detection. As for flammable/explosive gases/vapors, there are no official standards concerning dangerous concentrations, because these concentrations express physicochemical properties of gaseous mixtures which cannot be influenced by norms. Legislation tries only to prevent and reduce the consequences of inadequate handling of related hazards. Several concentration limits are relevant for flammable mixtures. They emerge from the characteristics of combustion processes for different stoichiometric composition of the fuel (gas/vapor) and oxidant gas (oxygen), ambient pressure and presence of other gases (nitrogen in the case of air) [441, 459-462]. A too lean or too rich flammable mixture will not burn. The lowest concentration of fuel for which the fueloxidant mixture still burns is known as the lower flammability limit (LFL), and the highest as the upper flammability limit (UFL). Often addressed are the lower explosion limit (LEL) and upper explosion limit (UEL). These terms are not very appropriate. Indeed, according to the combustion speed, one has deflagration for subsonic processes (speed of combustion lower than the speed of sound in air) and detonation for supersonic processes. Both can produce an explosion, that is, blowing up the vessel in which the combustion takes place because of increased pressure. A flammability/explosion limit is always an IDLH level, since it is immediately dangerous to life and health. The LFL and UFL for some analytes are given in Table 5. More comprehensive tables can be found in the references above [441, 459-462]. A general perspective on hazardous chemicals is given in the "Hazardous Chemicals Handbook" of Carson and Mumford [463].

\section{Overview of the target analytes detected by GGSs}

In many cases, the specific detection of gases and vapor with GGSs is based on the weak interactions between the analyte and the receptor, as previously accounted for in the section "Receptors for MST" of the first (published [1]) part of this review and in the section "Increasing the specificity of the receptors" above. Combined with chemical and geometric complementarity, these interactions can result in molecular recognition and, consequently, in increased sensitivity and 
Table 3 Some concentration limits for harmful gases. All data except IDLH values have been taken from the GESTIS databank (see the text). The IDLH values are reproduced from the online table of the NIOSH webpage. They are older than GESTIS data, that is, were published mainly before 1994 and only a few of them afterwards

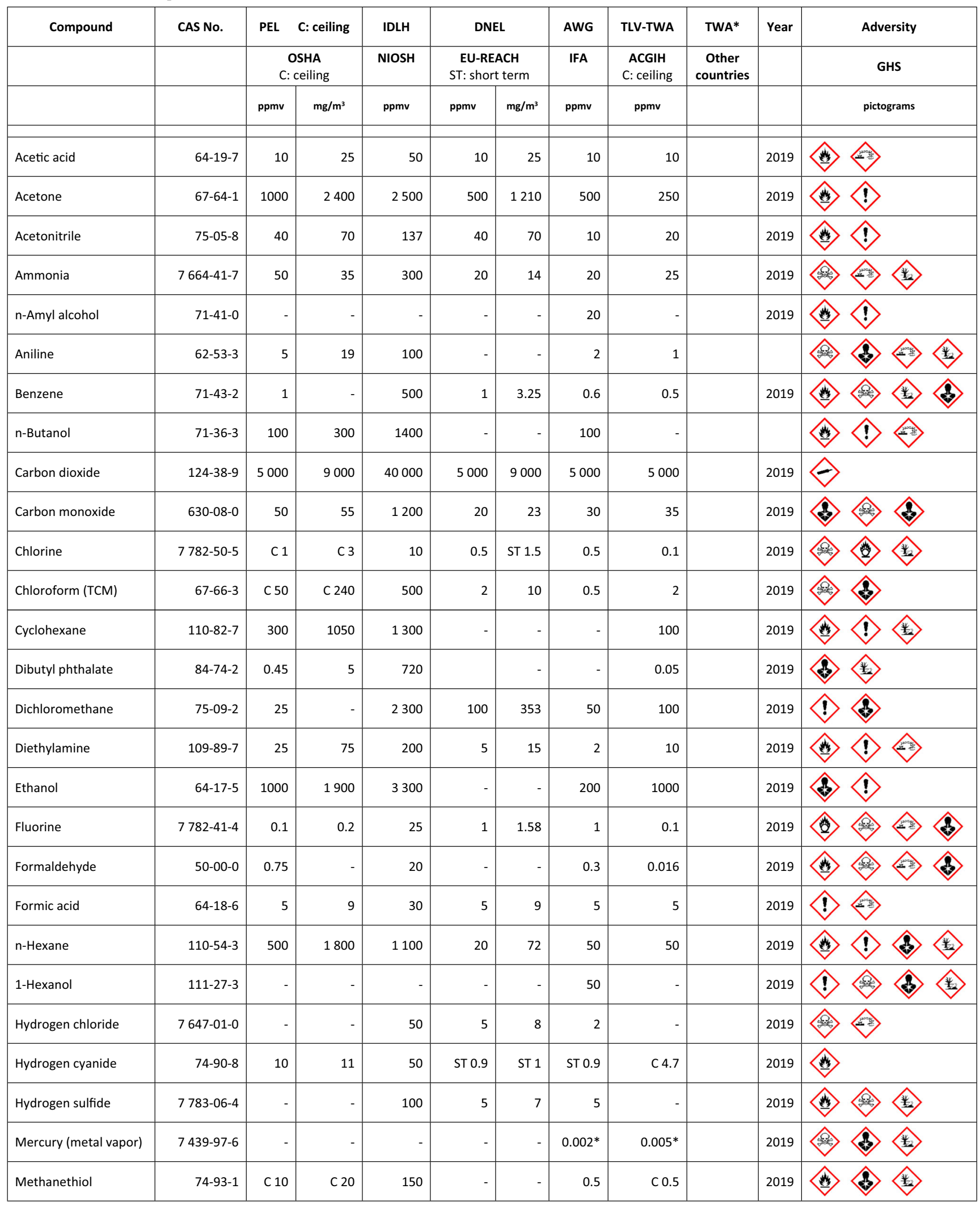


Table 3 (continued)

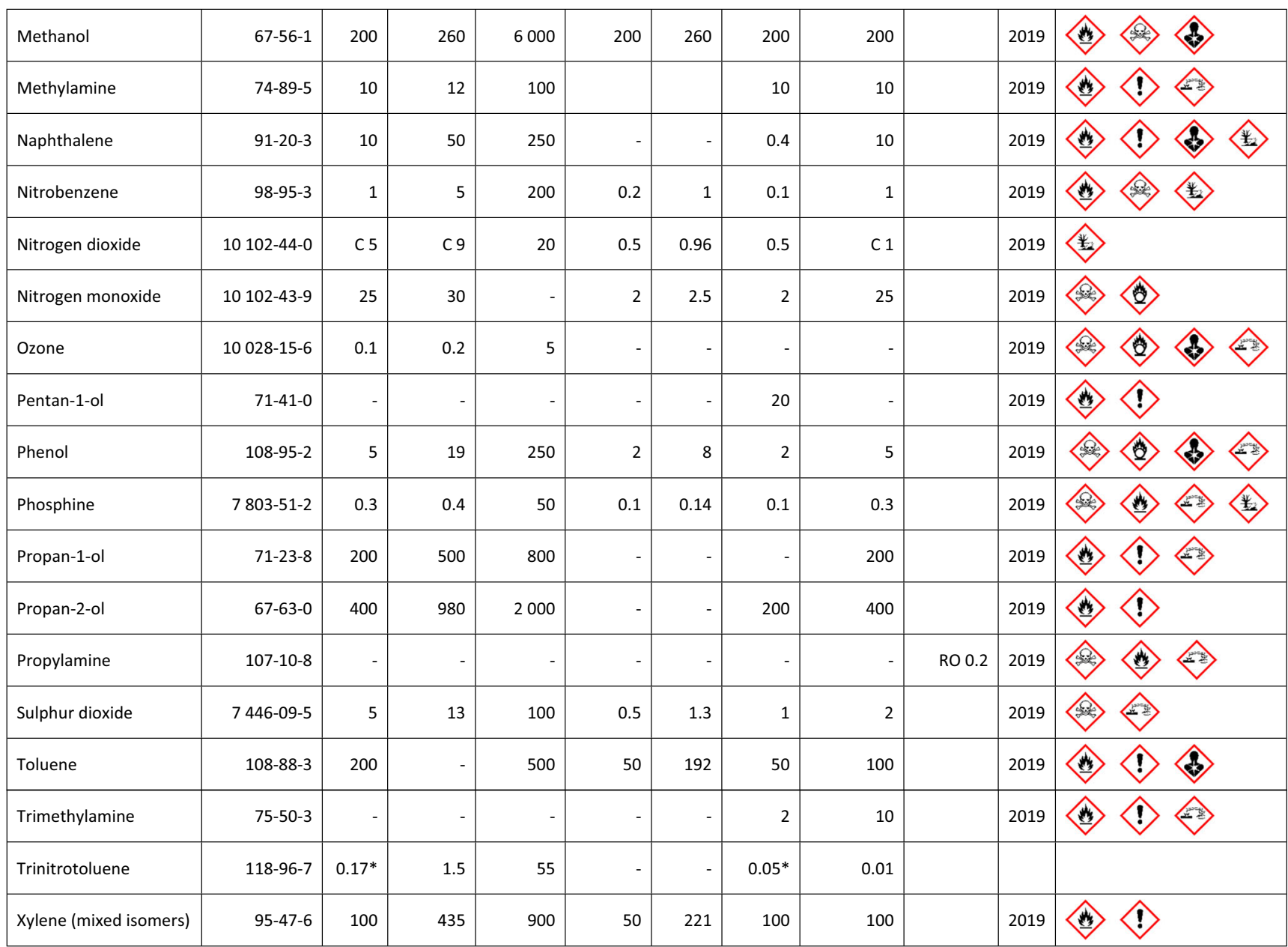

*If no value is available for the selected norms (the previous columns), a known limit value from another country given for reference. The value is also taken from the GESTIS database and the country symbol is placed before

**Values converted from $\mathrm{mg} / \mathrm{m}^{3}$ in ppmv by the review's authors, assuming ppbv level of $\mathrm{Hg}$ vapor to ideal gas

Table 4 Toxicity of selected CWAs and their simulants. Compiled from references [457] and [458]

\begin{tabular}{|c|c|c|c|c|c|c|}
\hline CWA & $\begin{array}{l}\text { CAS } \\
\text { CWA }\end{array}$ & $\begin{array}{l}\mathrm{LD} 50 \\
\mathrm{mg} / \mathrm{min} / \\
\mathrm{m}^{3}\end{array}$ & Simulant & Abbreviation & CAS Simulant & $\begin{array}{l}\text { Similarity } \\
\%\end{array}$ \\
\hline \multirow[t]{3}{*}{ Sarin (GB) } & \multirow[t]{3}{*}{$107-44-8$} & \multirow[t]{3}{*}{35} & Di-isopropyl fluorophosphate & DFP & $55-91-4$ & 87.5 \\
\hline & & & Di-isopropyl methyl phosphonate & DIMP & $1445-75-6$ & 75.0 \\
\hline & & & Dimethyl methyl phosphonate & DMMP & $756-79-6$ & 66.7 \\
\hline \multirow[t]{3}{*}{ Soman (GD) } & \multirow[t]{3}{*}{$96-64-0$} & \multirow[t]{3}{*}{35} & Di-isopropyl fluorophosphate & DFP & $55-91-4$ & 84.0 \\
\hline & & & Di-isopropyl methyl phosphonate & DIMP & $1445-75-6$ & 72.0 \\
\hline & & & Dimethyl methyl phosphonate & DMMP & $756-79-6$ & 64.0 \\
\hline \multirow[t]{3}{*}{ Tabun (GA) } & \multirow[t]{3}{*}{$77-81-6$} & \multirow[t]{3}{*}{70} & Diethyl ethyl phosphonate & DEEP & $78 \_38 \_6$ & 53.7 \\
\hline & & & Triethyl phosphate & TEP & $38-40-0$ & 52.5 \\
\hline & & & Diethyl 4-nitrophenyl phosphonate & Paraoxon & $311-45-5$ & 47.2 \\
\hline \multirow{3}{*}{$\begin{array}{l}\text { Distilled mustard } \\
\text { (HD) }\end{array}$} & \multirow[t]{3}{*}{$505-60-2$} & \multirow[t]{3}{*}{1000} & 2-Chloroethyl ethyl sulfide & CEES & $693-07-2$ & 64.7 \\
\hline & & & 2-Chloroethyl methyl sulfide & CEMS & $542-81-4$ & 52.9 \\
\hline & & & Chloroethyl phenyl sulfide & CEPS & 5 535-49-9 & 42.1 \\
\hline
\end{tabular}


Table 5 Flammability limits of selected gaseous mixtures with air. Values taken from reference [461]

\begin{tabular}{lllll}
\hline Compound & CAS & $\begin{array}{l}\text { LFL } \\
\text { Volume } \%\end{array}$ & $\begin{array}{l}\text { UFL } \\
\text { Volume } \%\end{array}$ & Remarks \\
\hline Acetone & $67-64-1$ & 2.6 & 13.0 & \\
Acetylene & $74-86-2$ & 2.5 & 100 & \\
Ammonia & $7664-41-7$ & 15.0 & 28.0 & \\
Benzene & $71-43-2$ & 1.3 & 7.9 & Both FL at \\
& & & & $100^{\circ} \mathrm{C}$ \\
$n$-Butane & $106-97-8$ & 1.8 & 8.4 & \\
Carbon monoxide & $630-08-0$ & 12.5 & 74.0 & \\
Ethane & Z4-84-0 & 3.0 & 12.4 & \\
Ethanol & $64-17-5$ & 3.3 & 19.0 & UFL at $60^{\circ} \mathrm{C}$ \\
$n$-Hexane & $110-54-3$ & 1.2 & 7.4 & \\
Hydrogen & $1333-74-0$ & 4.0 & 75.0 & \\
Methane & $74-82-8$ & 5.0 & 15.0 & \\
Methanol & $67-56-1$ & 6.7 & 36.0 & UFL at $60^{\circ} \mathrm{C}$ \\
$n$-Octane & $111-65-9$ & 0.95 & - & \\
Propane & $74-98-6$ & 2.1 & 9.5 & Both FL at \\
Toluene & $108-88-3$ & 1.2 & 7.1 & \\
& & & & \\
\hline & & & & \\
\hline
\end{tabular}

selectivity of the sensing process. In the context of the linear solvation energy relationship (LSER), numerous combinations of analyte-receptor material have been established, whose LSER parameters indicate the strength of different contributions to the recognition process [5, 464-466]. The chemical and physical properties of gases and vapors which might be relevant for gas sensing can be found in general chemistry textbooks [467-471] or technical literature [472-475]. The data from the technical literature will be used in the following for exemplifications without additional citation.

Volatile organic compounds VOCs are the most commonly addressed target analytes for GGSs. In many cases, the sensitivity of one sensor for several VOCs is reported. This feature, frequently seen as a useful characteristic, indicates however limited sensor selectivity. Previous sections ("Increasing the specificity of the receptors" and "Evaluating the performance of the gas sensors and sensor arrays") show how this drawback can be overcome by building up SAs and using chemometric methods.

Aromatic VOCs are stable compounds, with low reactivity, mainly detected through hydrogen bonds with the receptor. Monocyclic arene vapors are mostly reported. They are rather volatile (12.7 kPa for benzene, $3.8 \mathrm{kPa}$ for toluene and $0.79 \mathrm{kPa}$ for xylene at $25^{\circ} \mathrm{C}$ ). Toluene is often reported, as the TLV-TWA is rather high (100 ppmv) while the vapor pressure (VP) is not too great. Poly(aniline) emeraldine salt thin films doped with different acids (hydrochloric, dodecylbenzene sulfonic, 1,5naphthalene disulfonic) were deposited by dip coating on TSMRs as VOCs sensors [283]. Good sensitivity to aromatic
VOCs in dry nitrogen was obtained, but it decreased in the presence of humidity. The influence of oxygen on layer stability, which might be an issue for the given material, was not discussed. Siloxanes including triMethylSilane (3MS), DiEthoxyMethylSilane (DEMS) and OctaMethylCycloTetraSiloxane (OMCTS), deposited on TSMRs through PECVD by Sabahy et al., were able to detect toluene at concentrations below TLV-TWA [312]. These siloxane layers were hydrophobic, with contact angles larger than $95^{\circ}$, and response achieved with only a few $\mathrm{Hz}$ at $35 \% \mathrm{RH}$ variation. Even so, this reduced cross-sensitivity to humidity is still not enough for analyte concentrations below TLV, where the sensor responses to toluene are comparable to those of water. The same group of authors also used finite element theory to analyze the thermodynamics of the toluene sorption process in organic films [476]. Zhang et al. reported selective sensing of dibutyl phthalate (DBP) from 2ppbv to 30ppmv, with $0.66 \mathrm{ppbv}$ LDL and $3810 \mathrm{~Hz} /$ ppmv sensitivity, performed with TSMRs covered with Au-decorated $\mathrm{ZnO}$ porous microspheres [477], as illustrated in Fig. 22. The cross-sensitivity to dimethyl and diethyl phthalates is significant, but the cross-sensitivity to other VOCs appears to be reduced in the plots, as they are given at levels much lower than the TLV-TWA of these compounds (see Fig. 22). The effect of humidity, which is not addressed, might also limit sensor performance. Öztürk et al. utilized Pd-doped $\mathrm{ZnO}$ nanorods electrochemically deposited on TSMRs to detect xylene and other VOCs [313]. The response to xylene was highest (3.3 Hz/ppmv, 5 ppmv LDL), but the sensitivity to other VOCs (mainly ethanol and propanol) was also good, leading to a critical selectivity issue. Very good sensitivity for toluene (LDL=1ppmv, that is $1 \%$ from TLV-TWA) was obtained by Yamagiwa et al. with both Q-TSMR and Si micro-cantilevers coated with self-assembled MOFs, but with reduced specificity, due to a strong response to ethanol [175]. Selectivity problems were also encountered by Kumar et al. in detecting toluene with phthalocyanine-covered Q-TSMRs, because the sensitivity to xylene was practically the same [115]. Bachar et al. compared chemoresistive and gravimetric (TSMR) SAs based on heavy polycyclic aromatic hydrocarbons [231]. The gravimetric SA exhibited different sensitivity for different compounds, while the chemoresitive SA did not. Both arrays were evaluated for alkanes, alcohols, mesitylene, ether and water with chemometric methods. They were able to separate the polar compounds (mainly alcohols) from the nonpolar ones (mainly alkanes), with the help of DFA, in humidity background from $5 \%$ to $80 \% \mathrm{RH}$.

Aliphatic VOCs. Several classes of gases and vapors are included here. The aliphatic alcohols are polar and volatile (VP is $13 \mathrm{kPa}$ for methanol and $0.65 \mathrm{kPa}$ for butanol), with different degrees of toxicity. The aldehydes are less polar. Formaldehyde is very toxic (TLV-TWA of $16 \mathrm{ppbv}$ ) and gaseous at room temperature, while acetaldehyde boils at just $20^{\circ} \mathrm{C}$ and is not as toxic as formaldehyde. Being slightly polar and gaseous or 
Fig. 22 Dibutyl phthalate detection with Q-TSMRs coated with Au-decorated $\mathrm{ZnO}$. (a) Calibration curve. (b) Crosssensitivity to VOCs. DEP and DMP stand for diethyl and dimethyl phthalate, respectively. Reproduced with the kind permission of the authors and MDPI from reference [477]

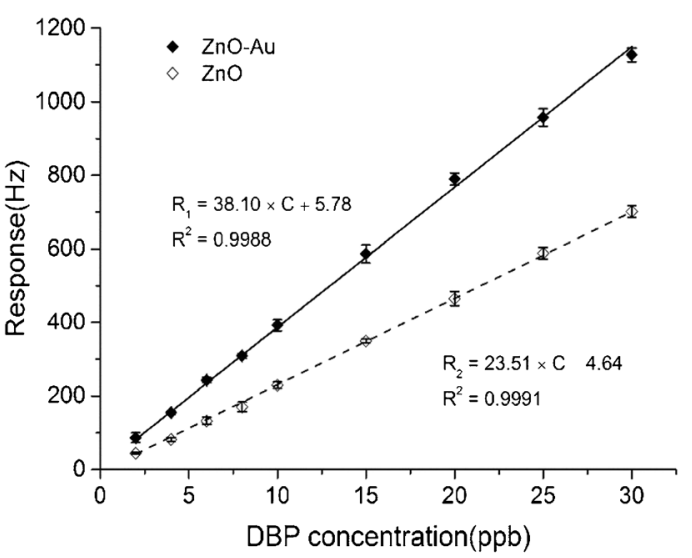

a

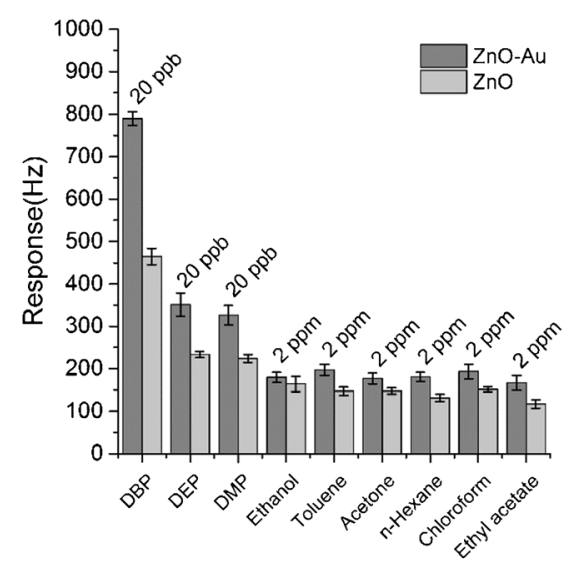

b very volatile, the amines are toxic (TLV-TWA in $10 \mathrm{ppmv}$ range). The alkanes are nonpolar and not very toxic but easily flammable. They are permanent gases for low molecular mass (until butane) at room temperature. Yang and He performed formaldehyde detection with graphene oxide coated on quartz TSMRs in the presence of humidity [478]. The specificity was good, except for ethanol, for which relevant cross-sensitivity was observed. For the same analyte, Wang et al. proposed a TSMR GGS having a novel copper (II) complex as sensing layer, which was very sensitive (LDL only $1.3 \%$ from PEL and $62 \%$ from TLV-TWA) and quite selective [120]. The crosssensitivity to humidity was, however, significant for atmospheric values. Therefore, almost the same group of authors tested a hydrophilic poly(dopamine) passivated against humidity by a super-hydrophobic poly(n-octadecylsiloxane) film as sensing material for formaldehyde [187]. The influence of water was drastically reduced, but the detection limit decreased to 0.5 ppmv (value estimated by the authors of this review). Formaldehyde detection with hollow mesoporous silica spheres (HMSS) functionalized with poly(dopamine) (PDA) was reported by Zong et al. [117]. The authors employed QTSMRs MST operating at $10 \mathrm{MHz}$. The sensor performance is displayed in Fig. 23. A review of TSMR-based sensors for formaldehyde is provided by reference [479].

Alcohols have been reported mainly in conjunction with practical applications such as VOC discrimination with LDA [240] and food quality evaluation [249, 480]. Amines have generated much interest. Das et al. demonstrate the use of polymerized castor oil for the detection of aliphatic amines (LDL of $10 \%$ from TLV-TWA) [281]. The sensitivity decreased slightly with amine molar mass, leading only to class recognition. Ammonia produces significant interference. $\mathrm{Li}$ and $\mathrm{Chu}$ obtained good sensitivity (see Fig. 24) to propylamine in measurements performed on Q-TSMRs coated with ionic liquids (5.4 ppbv LDL and 2000 $\mathrm{Hz} / \mathrm{ppm}$ sensitivity) [189]. The sensors were quite sluggish. Their response was not affected by traces of water, but a test of cross-sensitivity to humidity was not considered.
Zhang et al. [182] and Chen et al. [481] reported on trimethylamine (TMA) detection with Q-TSMRs and GO-based layers ( $\mathrm{GO} /$ chitosan and $\mathrm{GO} / \mathrm{Cu}_{2} \mathrm{O}$ nanocomposite, respectively). Both receptor materials were sensitive $(4.8 \mathrm{~Hz} / \mathrm{ppmv}$ and $8.9 \mathrm{~Hz} /$ ppmv, respectively), but the $\mathrm{GO} / \mathrm{Cu}_{2} \mathrm{O}$ nanocomposite had a much lower LDL (230ppbv) than that of GO/chitosan (1.3 ppmv). The cross-sensitivity to other amines was high, but the response to other VOCs was limited in the case of GO/chitosan films. The strong influence of humidity was a common issue for both sensors, acknowledged by the authors, who informed the reader that they would continue the investigation in this regard. To reduce crosssensitivity to water, Chen et al. produced composite films of hexanal-imprinted MIPs and hydrophobic silica NPs [180]. The efficiency of the approach can be observed in Fig. 25. Methane detection with cryptophane-A/E films was reported by Wang et al. and Shen et al., respectively, using Q-SAW transducers at $300 \mathrm{MHz}$ and $204 \mathrm{MHz}$ [482, 483]. The sensitivity was sufficient to measure well below the LFL of $5 \%$. However, in the case of cryptophane-A, 70\% RH gave the same response as LFL methane concentration. For cryptophane-E, the cross-sensitivity to humidity was not reported. Molecularly imprinted polymers (poly(methyl methacrylate)) have shown good sensitivity for terpenes, but with significant cross-sensitivity to humidity [91].

Chemical warfare agents and other special analytes. As specified above, the detection of CWAs has been investigated on simulants, most of them included in Table 4. TSMR with mesoporous $\mathrm{TiO}_{2}-\mathrm{SiO}_{2}$ functionalized with $\mathrm{p}$-hexa-fluoroisopropanol aniline (HFIP) showed acceptable sensitivity and specificity towards the nerve agent simulant DMMP [118]. The influence of humidity was not discussed. Sulfur mustard and DMMP sensors based on composite materials were reported by Lal and Ziwari [179] (see the subsection 'Composite, polymorph and unusual receptor materials' above) and Yang et al. [484]. Di Pietrantonio et al. used laser-induced forward transfer (LIFT) to deposit thin polymer layers on Q-SAW, which were very sensitive to the simulant DMMP [485]. The experiments were repeated on real CWA (sarin 

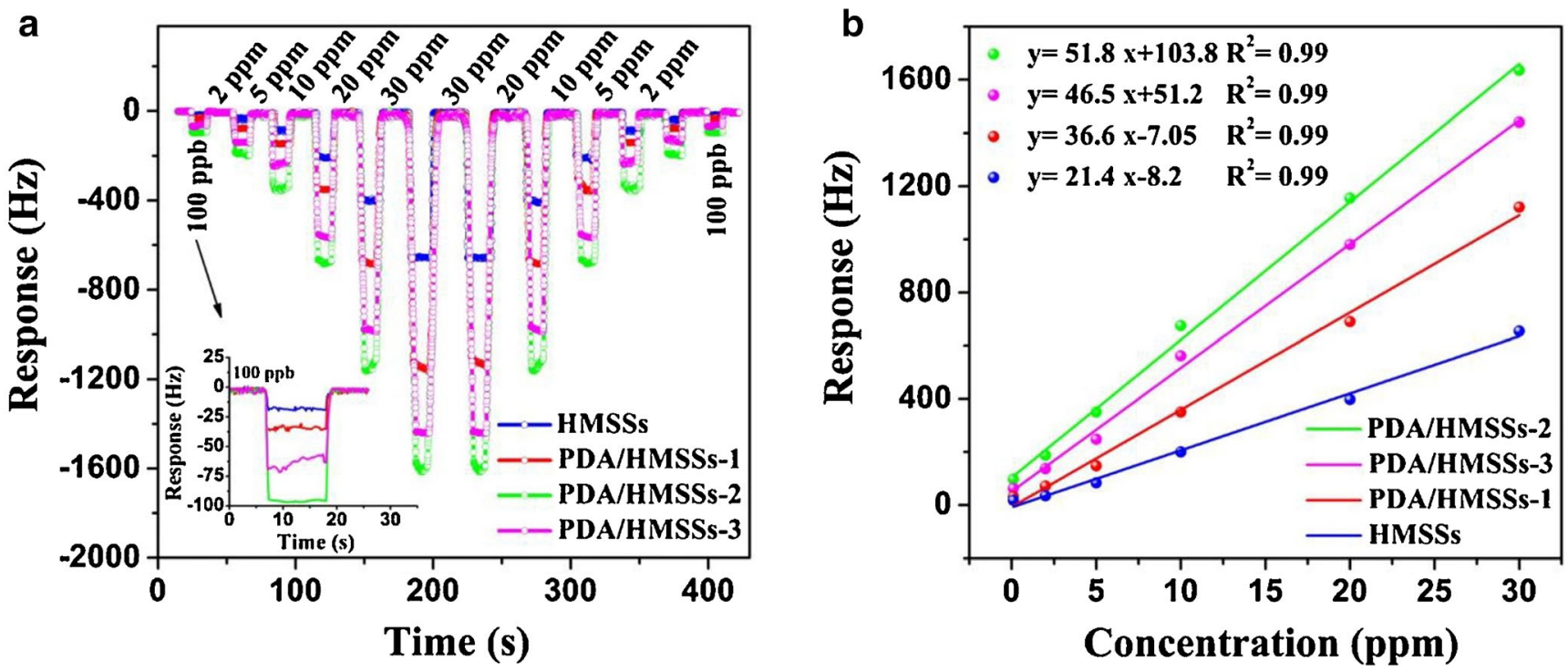

Fig. 23 Formaldehyde detection with PDA-functionalized HMSS. (a) Dynamic response. (b) Calibration curves. Reproduced with the kind permission of Elsevier B.V. from reference [117]

in this case) with even better performance $(649 \mathrm{~Hz} / \mathrm{ppmv}$ sensitivity and 150 ppbv LDL). Despite certain selectivity displayed by different polymers, a dedicated cross-sensitivity test was not performed, but was planned. Employing a Love-SAW array coated with six dissimilar polymers, Matatagui et al. also achieved very good performance for CWA detection $(40,200 \mathrm{~Hz} /$ ppmv sensitivity and 40 ppbv LDL) [229]. Moreover, they were able to discriminate the tested agent simulants, with scores in the first two PCs nicely clustered for the whole concentration range. In a later investigation by almost the same authors, a GO sensing layer was chosen [486]. The good sensitivity of the device is demonstrated by the plots in Fig. 26. Chen et al. achieved successful detection of DMMP with a wireless Q-TSMR having both resonance frequency and dissipation output [487]. The MST was covered by hollow ball-like indium oxide and enabled $2.1 \mathrm{~Hz} / \mathrm{ppmv}$ sensitivity and an LDL below 5 ppmv. The vapors of explosive nitroaromatic compounds 2,4-dinitrotoluene (2,4-DNT) and trinitrotoluene (TNT) were specifically recognized by TSMRs coated with methylated mesoporous silica [437]. This approach was already addressed in "Unconventional theoretical and numerical approaches to sensor data analysis" above. The same types of compounds were detected by Eslami and Alizadeh with poly(pyrrole) (PPy)-bromophenol blue (BPB) layers on Q-TSMRs [488]. Comparative responses for several analytes including TNT, [3-nitrooxy-2,2-bis (nitrooxymethyl)propyl] nitrate (PETN), 1,3,5-trinitroperhydro1,3,5-triazine (RDX) and octahydro-1,3,5,7-tetranitro-1,3,5,7tetrazocine (HMX) given by the selected sensing materials are included in Fig. 27. The detection of toxic hydrogen cyanide with $\mathrm{CuO}$ nanoparticles appears promising from the contribution of Yang et al. [489]. The material seems to have certain selectivity towards $\mathrm{HCN}$ due to the unexpected positive gravimetric response of the coated TSMRs, opposite to the "normal" response to the other tested analytes, which was negative. The authors attributed the sensor behavior to mass loss during recognition redox reaction.

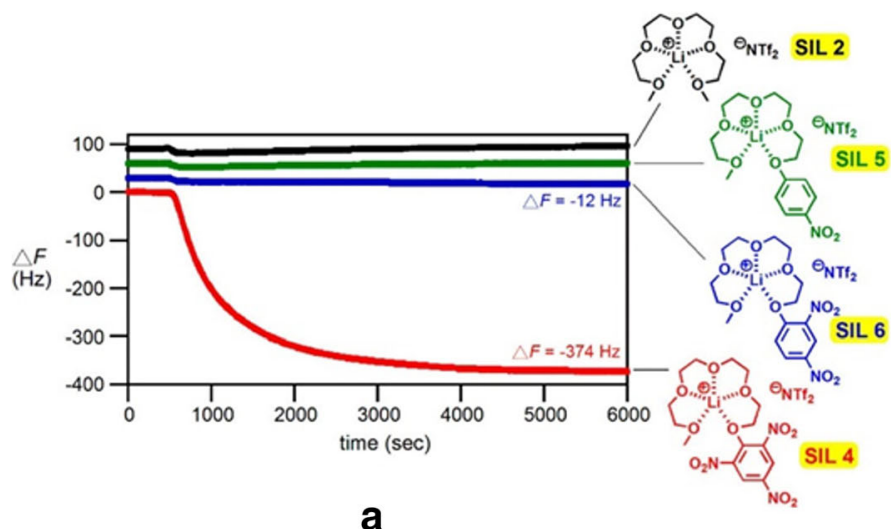

a

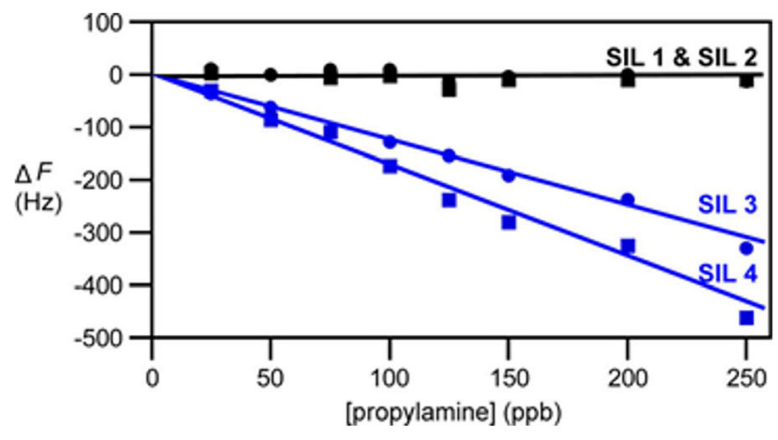

b

Fig. 24 Propylamine detection with $9 \mathrm{MHz}$ Q-TSMRs coated with ionic liquids. (a) Dynamic response. (b) Calibration curves. Reproduced with the kind permission of ACS Publications from reference [189] 

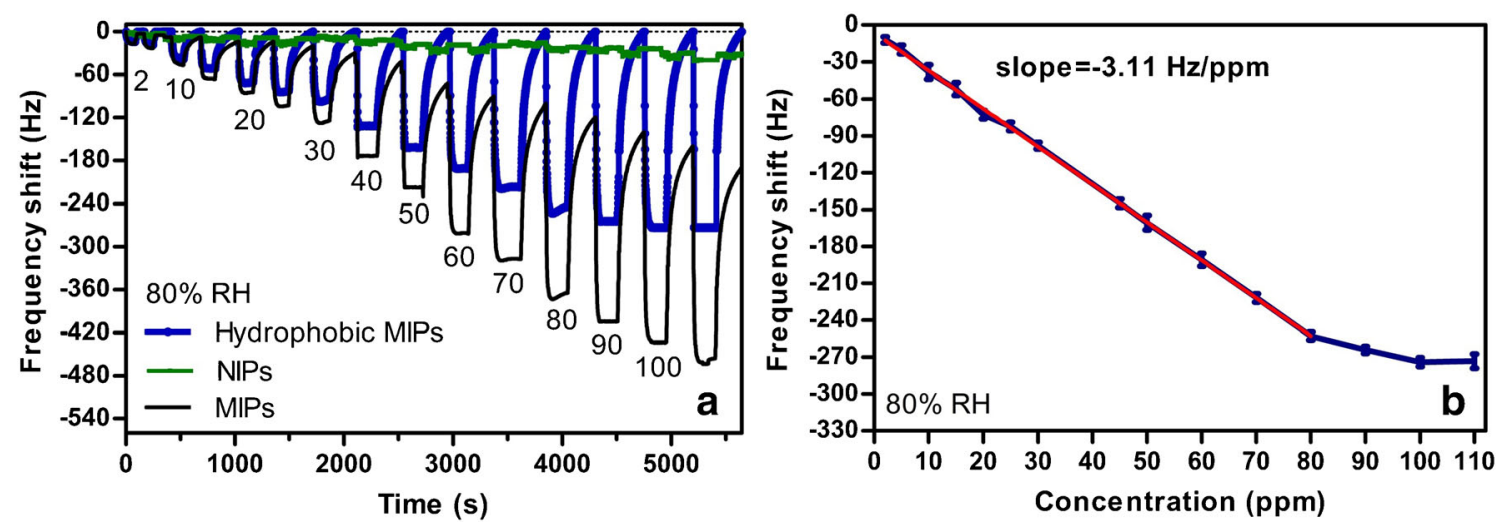

Fig. 25 Hexanal detection with Q-TSMRs coated with hydrophobic MIP-SiO 2 NP composite. (a) Dynamic response. NIP and MIP signals are provided by the non-imprinted and imprinted materials alone. (b) Calibration curve. Reproduced with the kind permission of Elsevier B.V. from reference [180]

However, the response in atmosphere containing both analyte and interfering gas was not evaluated, despite possible compensation of the effects. Also, the humidity was kept constant, at a low level of $10 \%$, hindering a more realistic characterization. In a follow-up paper, the authors reconsidered the investigation and obtained high cross-sensitivity to humidity [490]. Precursors of illicit compounds have been recognized and detected with high-frequency Q-TSMRs (195 MHz) coated with five microporous triptycene-based affinity materials [491]. Prantl et al. did not report metric and crosssensitivity data, but the approach seems interesting.

Inorganic gases. From the class of inorganic gases, those most investigated are the hazardous gases $\left(\mathrm{CO}, \mathrm{CO}_{2}, \mathrm{NO}_{2}, \mathrm{SO}_{2}, \mathrm{HN}_{3}\right.$, $\mathrm{H}_{2} \mathrm{~S}$ ) whose presence in the atmosphere is of major concern. $\mathrm{CO}$ detection has been addressed several times by researchers in the field. Ippolito et al. employed for this purpose a heated SAW coated with $\mathrm{In}_{2} \mathrm{O}_{3}$ [304]. Its operating principle was already addressed in the paragraph "SAW transducers" of the first review part [1]. $\mathrm{H}_{2}, \mathrm{NO}_{2}$ and $\mathrm{CO}$ were detected at room

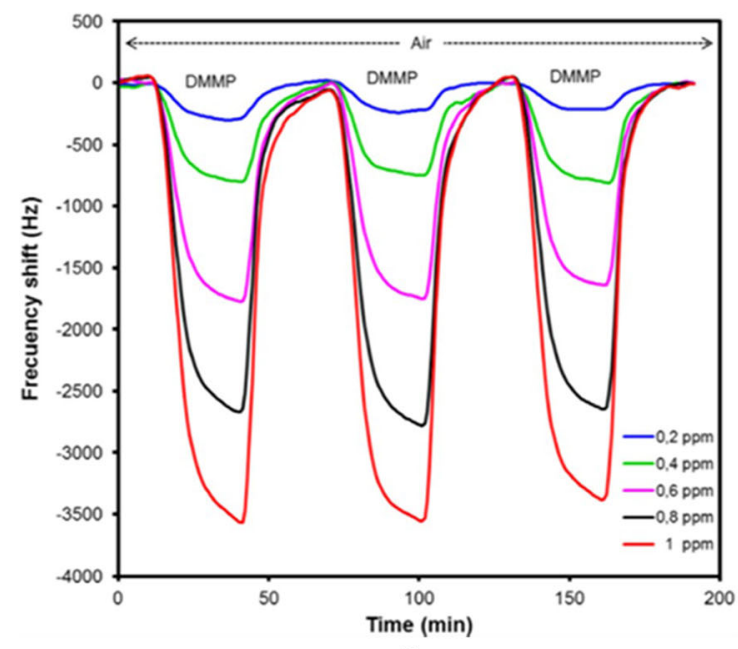

a temperature when the same type of SAW was coated with a poly(aniline)// $/ \mathrm{I}_{2} \mathrm{O}_{3}$ nanofiber composite [492]. Bayram et al. detected $\mathrm{CO}$ with ferrocene-branched chitosan derivatives on quartz TSMR, but with rather low sensitivity [493]. Tian et al. obtained acceptable $\mathrm{SO}_{2}$ and $\mathrm{NO}_{2}$ sensitivity and selectivity with electropolymerized ring-substituted (2-methyl, 2-metoxy) poly(aniline)s on TSMRs [494]. However, it was not possible to evaluate concentrations separately if both analytes were present in the sample. Using micro-cantilevers coated with metal organic framework crystals of Ni-MOF-74 (also Fe, Mn and $\mathrm{Mg}$ tested with less success), $\mathrm{Lv}$ et al. were able measure $\mathrm{CO}$ concentrations below 10ppbv due to the large BET surface area of the receptor material $\left(1150 \mathrm{~m}^{2} / \mathrm{g}\right)$ [495]. The signals were strong, but the response/recovery times were also long, due to rather high adsorption enthalpy of $\sim 53 \mathrm{~kJ} / \mathrm{mol}$ (see Fig. 28). Good repeatability, selectivity, long-term stability (sensitivity degradation of $1 \%$ in 6 months) and manufacture reproducibility (12\% spread over 4 samples) indicated a good sensor performance. The calibration curves were strongly nonlinear, favoring the

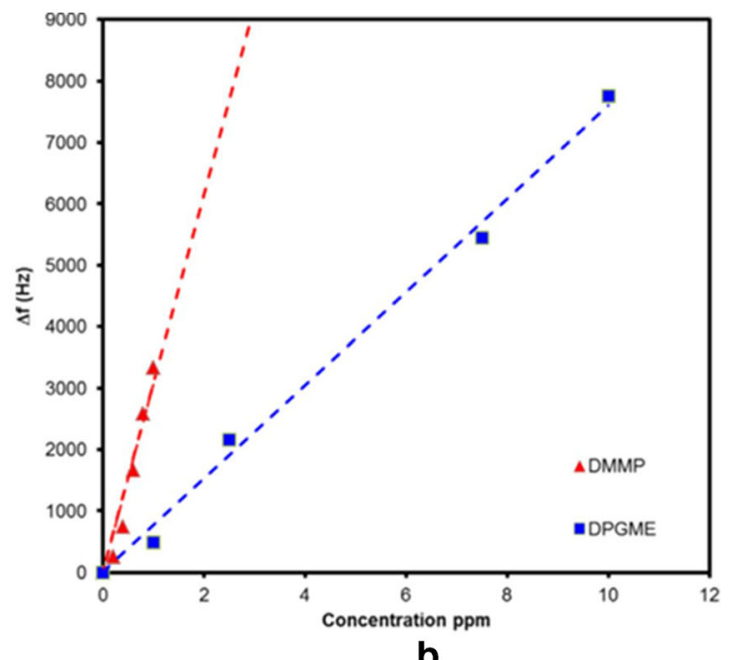

b

Fig. 26 Dynamic response (a) and calibration curve (b) of a GO-coated Love-SAW sensor exposed to CWAs. Reproduced with the kind permission of Elsevier B.V. from reference [486] 

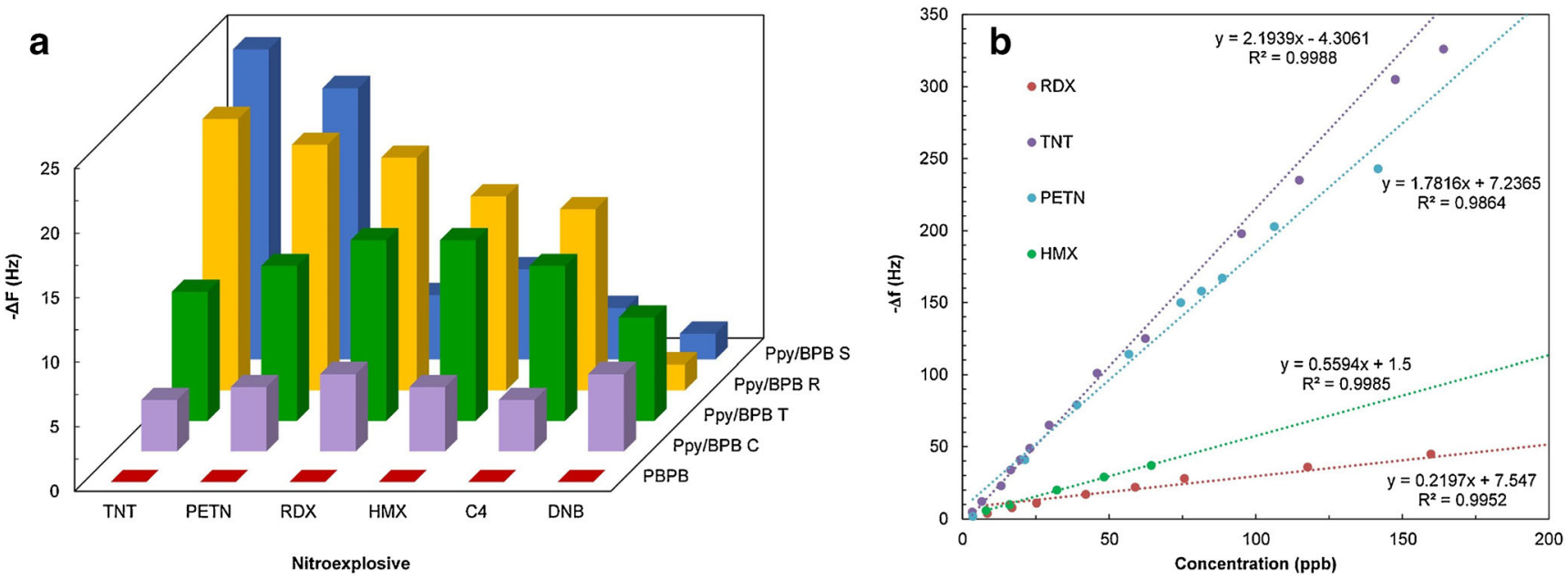

Fig. 27 (a) "The response of differently prepared sensors including poly BPB (PBPB), PPy-BPB co-polymer (PPy/BPB C), PPy/BPB thin film (PPy/BPB T), PPy/BPB nanospheres (PPy/BPB S) and PPy/BPB nanorods (PPy/BPB R) toward nitroexplosives" (original caption). (b) The

low concentration levels. The influence of humidity, however, was not addressed. Wang et al. detected (see Fig. 29) low ammonia levels (60 ppbv LDL) with high sensitivity $(100 \mathrm{~Hz} / \mathrm{ppmv})$ with $10 \mathrm{MHz}$ Q-TSMRs covered by La-doped framework AlPO-5 [158]. Cross-sensitivity to humidity, carbon dioxide, acetone and nitrogen dioxide were discussed. Hydrogen sulfide detection has been addressed by two authors. Asad et al. evaluated $\mathrm{H}_{2} \mathrm{~S}$ concentrations between 5 and 200 ppmv using a $\mathrm{LiNbO}_{3} \mathrm{SAW}$ operated at $104 \mathrm{MHz}$ and room temperature [136]. The sensing material, Cu-NP-SWCNT, was drop-cast on the MST surface, and conferred high sensitivity $(3750 \mathrm{~Hz} /$ ppmv) and reasonable selectivity. Responses $\sim 10$ times lower than those for hydrogen sulfide were obtained for ethanol, hydrogen and acetone. At operating temperatures below $100{ }^{\circ} \mathrm{C}$, the sensor response was influenced by humidity, but thanks to the high-temperature piezoelectric material of the transducer, operation at over $100^{\circ} \mathrm{C}$ could be considered and the water vapor removed from the sensor surface. The $\mathrm{H}_{2} \mathrm{~S}$ sensor prepared by $\mathrm{Li}$ et al. is based on a Q-SAW-MST, spin-coated with $\mathrm{CuO}$

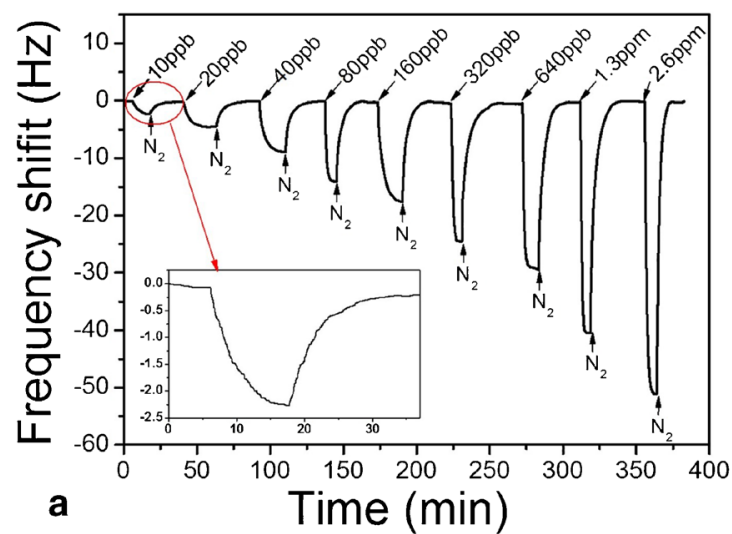

calibration curves of the PPy-BPB/QCM sensor for TNT, PETN, RDX and HMX with low LDLs (500 ppt for TNT, 800 ppt for PETN, 1 ppbv for RDX and $2 \mathrm{ppbv}$ for HMX). Reproduced with the kind permission of Elsevier B.V. from reference [488]

prepared by a sol-gel route [496]. It has $4 \mathrm{kHz} / \mathrm{ppmv}$ sensitivity and 0.5 ppmv LDL in a humid environment. The authors tried to explained this behavior through the shift in the equilibrium of the reaction $\mathrm{H}_{2} \mathrm{~S}+\mathrm{CuO} \rightleftharpoons \mathrm{CuS}+\mathrm{H}_{2} \mathrm{O}$ due to increased water concentration, but they produced no evidence in this respect. The cross-sensitivity to VOCs and inorganic gases was low. Hydrogen is not toxic, but the evaluation of its concentration in the range of LFL (4\%) has fire safety relevance. Viespe and Miu reported good hydrogen detection (59 ppmv LDL and $0.51 \mathrm{~Hz} /$ ppmv sensitivity) with $\mathrm{Pd} / \mathrm{Zn}$ bilayers produced by pulsed laser deposition (PLD) on 70MHz Q-SAWs [497]. Using only Pd films on silicon micro-cantilevers and static bending operating mode, McKeown et al. detected 250 ppmv of hydrogen in air [498].

Humidity. Humidity is the third/fourth component of atmospheric air (around 1\% volume for 50\% RH at room temperature, like argon). It is not toxic, but water vapor concentrations that are too low or too high cause discomfort. Additionally, it is

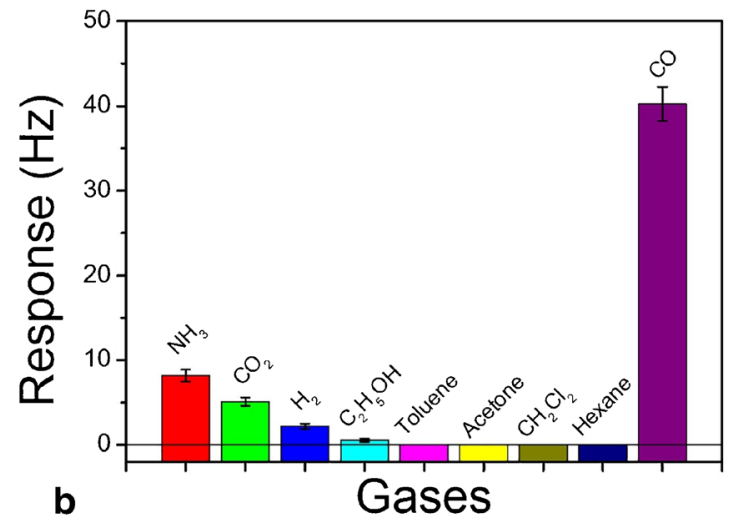

Fig. 28 Dynamic response to carbon monoxide (a) and cross-sensitivity (b) of a micro-cantilever coated with Ni-MOF-74. Reproduced with the kind permission of Elsevier B.V. from reference [495] 

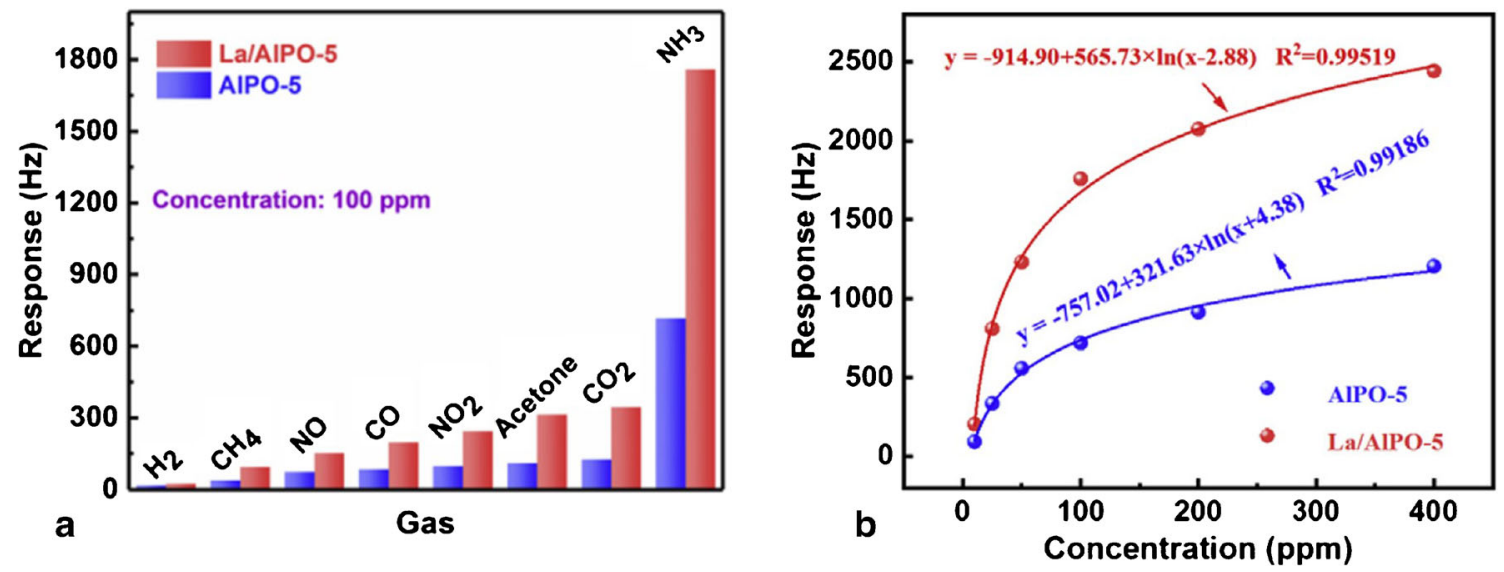

Fig. 29 The responses to different analytes (a) and the calibration curves for ammonia (b) of Q-TSMRs coated with AlPO-5 and La-doped AlPO-5. Reproduced with the kind permission of Elsevier B.V. from reference [158]

the main interferent in the case of environmental gas sensors. This issue, as already addressed at the beginning of the first review part [1], emerges from naturally large values of absolute humidity in the atmosphere, much larger than the detected levels of almost any other analyte. Traditionally, one evaluates the relative humidity, that is, the ratio of the water partial and saturation vapor pressure at the measurement temperature. This brings additional inconvenience, because the absolute water concentration for a given relative concentration increases with temperature following Antoine's law (as does the saturation vapor pressure) [469]. Although polymer capacitive sensors are the state of the art in humidity sensing, several studies still investigate sensors based on MSTs [499]. This interest is probably because most of the plain sensing materials have large dispersion interaction, which favors the detection of the analyte with highest concentration, usually humidity. Yuan et al. used spray coating to deposit thin nanocomposite layers of poly(ethylenimine)-graphene oxide on quartz TSMRs which were sensitive to humidity [186]. The resulting sensors exhibited low cross-sensitivity to formaldehyde, carbon dioxide, ammonia and sulfur dioxide, reduced response and recovery times, and good long-term stability. TSMRs based on a graphene oxide/tin dioxide/poly(aniline) (GO/SnO2/PANI) composite fabricated via in situ oxidative polymerization by Zhang et al. showed wide pore size distribution and large surface area but low contact angle, being well suited for humidity detection [287] (see Fig. 30). Xuan et al. achieved good humidity estimation with GO deposited by spin coating or drop casting on $140 \mathrm{MHz}$ and $225 \mathrm{MHz} \mathrm{ZnO}-\mathrm{SAWs}$ manufactured on glass substrates [500]. The sensors had exponential calibration curves, which could be linearized for low humidity $(7 \mathrm{~Hz} /$ ppmv $=1.448 \mathrm{kHz} / \% \mathrm{RH}$ in this range). Many authors have reported on humidity sensors based on different materials such as mesoporous $\mathrm{SnO}_{2}-\mathrm{SiO}_{2}$ [501] or porous poly(methyl methacrylate) [502]. The measurement of humidity at atmospheric concentrations seems to be an easy task, even in the presence of other gases, because of the large absolute values of water concentration (see above and the paragraph "The performance of GGSs" of the first review part), which leads to an increase of the relative response to humidity with respect to almost any other compound at the TLV-TWA level. However, condensation, possibly connected to the presence of interfering gases, can result in significant inaccuracy [503]. In order to facilitate sensor calibration at low humidity, Tsukahara et al. developed an original method to produce water vapor at the ppmv level [504], which used the controlled retention of water on the inner surface of a pipeline.

\section{Targeted applications}

The reports on GGSs seldom specify only a targeted application for the sensors studied. As shown in the previous paragraph, most of authors indicate the main analyte and sensor performance, leaving open the choice for its future use. There are, however, several exceptions. Among these, food quality control, medical instrumentation, detection of very dangerous industrial emissions and ambient air quality are the most relevant.

Freshness, quality and flavor of food Traditionally, quality assessment in the food industry has been performed by panels of human experts. Researchers in the field of gas sensing have tried to automate and simplify this process by building dedicated SAs and evaluating their data with chemometric methods. Some approaches are based on GGSs only. Sharma et al. analyzed the headspace vapor containing linalool to discern the flavor of black tea [436]. The pseudo-chemometric procedure was already explained in the section "Evaluating the performance of the gas sensors and sensor arrays", last paragraph ("Unconventional theoretical and numerical approaches to sensor data analysis"). The fermentation of black tea has been monitored through a similar approach [505]. TSMRs coated with poly(dimethyl siloxane) were employed to evaluate the ripening degree of mango fruits after the released amount of 

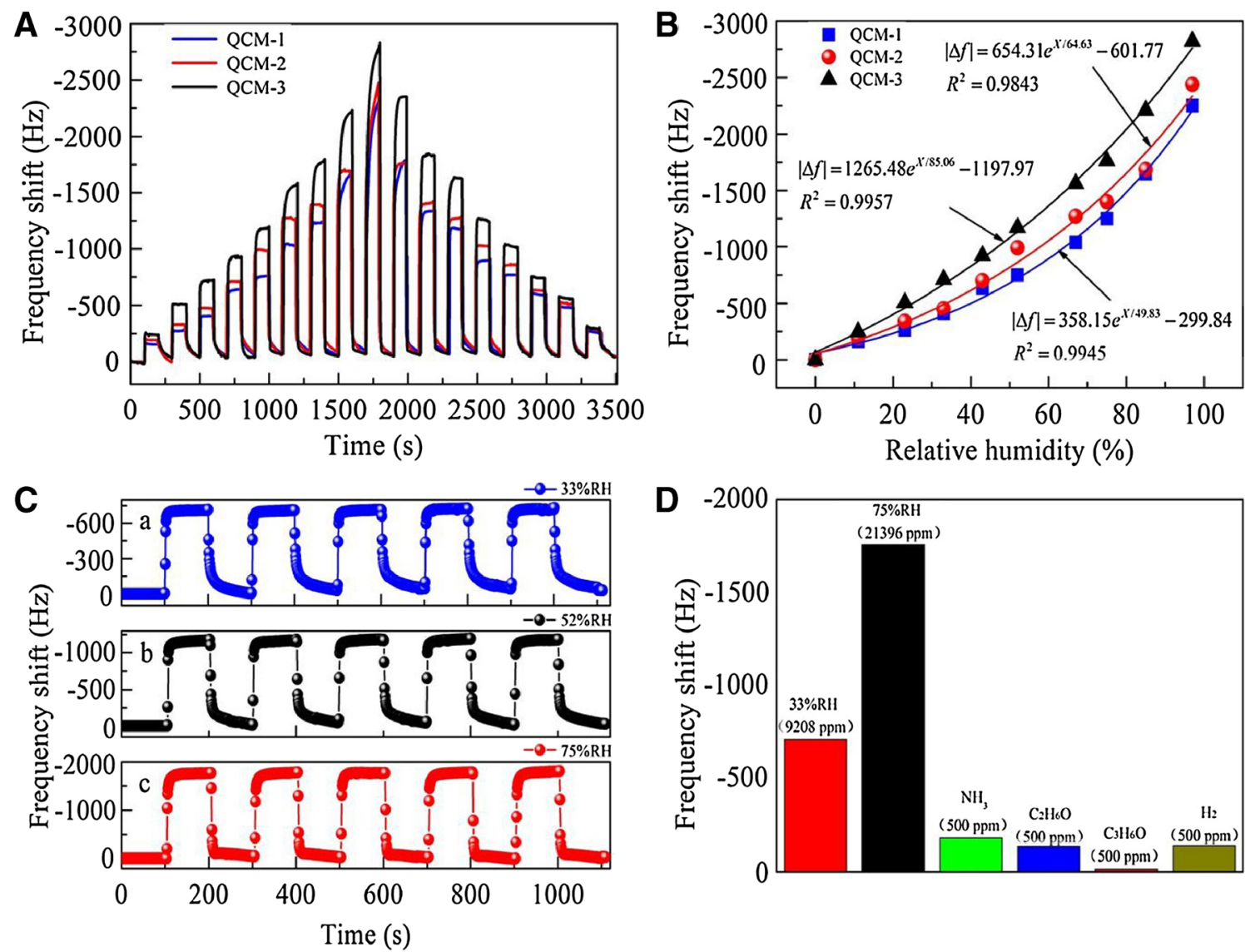

Fig. 30 (a) "Dynamic frequency shift of the three QCM sensors switching under various RH levels." (b) "Frequency shift of the three QCM sensors as a function of RH." (c) "Repeatability of the QCM-3 sensor switched from $0 \% \mathrm{RH}$ to $33 \% \mathrm{RH}, 52 \% \mathrm{RH}$ and $75 \% \mathrm{RH} . "$ (d)

3-carene [506]. The assessment of virgin oil quality through gravimetric arrays was reported by Escuderos et al. [338]. A percentage of $91.7 \%$ correct classification was obtained in the first two PCA components. Compagnone et al. used two types of TSMR-based GGSs for quality control of chocolate [103]. Both the porphyrin and the gold-nanoparticle-peptide GGS were able to separate the batches with standard flavor from the rest in the frame of PLS-DA. With peptide/gold nanoparticle Q-TSMR arrays, it was possible to detect/discriminate food aromas [51]. Toniolo et al. estimated food quality by means of a Q-TSMR array coated with ionic liquids [342], while Cui et al. assessed the degree of freshness/storage time of fish, eggs and mangos [480] (see Fig. 31). The TSMRs were covered with poly(pyrrole) $/ \mathrm{TiO}_{2}$ nanocomposite assembled through layerby-layer self-assembly. Using ZnO NPs modified with four different molecularly modeled peptides, Mascini et al. produced a Q-TSMR array with low sensitivity to humidity which could separate VOCs relevant for fruit juice identification [249]. The PCA biplot in Fig. 32 demonstrates the right choice of the sensing materials (according to the loadings of the four sensors indexed as WHVSC, LAWHC, IHRIC and TGKFC) and the good separation of the juice scores in the first two PC

"Selectivity of the QCM-3 sensors towards RH and various gas species." (Original caption; here RH stands for relative humidity). Reproduced with the kind permission of Elsevier B.V. from reference [287]

planes. The freshness of eggs was checked by Deng et al. using a Q-TSMR SA dip-coated with different receptor materials (multi-walled carbon nanotubes, graphene, copper oxide and poly(aniline)) [343]. The PLSR and PLSR/KPCA plots of the predicted versus actual storage times of eggs were already given as an example in Fig. 18, and confirm the success of the attempt. The potential for a polymer-functionalized SAW SA to recognize coffee flavor through the scores in the first two PCs was analyzed by Barié et al. [507].

Medical and biological applications The increasing need for noninvasive and cheap investigative tools for medical use has led to growing interest in gas sensor research in this field. Because the gaseous markers of diseases are released in very small amounts from clinical samples, sensors with very low LDLs and high sensitivity are suitable. In the case of breath analysis, the sample is taken from the patient exhalation and contains water vapor almost at saturation $(66,000 \mathrm{ppmv}$ at human body temperature). These special operating conditions can seldom be fulfilled by individual sensors, and SAs and chemometric methods are typically employed to gather the medical information. Ogimoto et al. prepared porous films 
comprising silica nanoparticles/poly(allylamine hydrochloride) (SiNPs/PAH) infused with poly(acrylic acid) (PAA) on Q-TSMRs to detect ammonia in breath analysis as a biomarker of renal insufficiency or hepatic dysfunction [119]. Using two transducers, one coated with SiNPs/PAH and the other with SiNPs/PAH/PAA, the authors built a differential sensor system able to subtract the influence of breath humidity (see Fig. 33) and to measure the ammonia concentration of the physiological samples. A breath analysis array of six QTSMRs coated with organic and composite nanometric films was devised and tested by Selyanchyn et al. [246]. Because the sensor system considers the influence of the temperature and exhalation flow, extended health control can be performed. Jha and Hayashi succeeded in detecting organic acids or aldehyde in body odor with a Q-TSMR array covered with molecularly imprinted polymers [247, 248]. The experimental data resulted in good clustering in the first two PCA components. Also, diseases which are not "visible" in exhalation can be detected with GGSs, like urinary tract cancer. Bernabey et al. employed an EN based on eight Q-TSMR sensors with dissimilar porphyrin receptors to discriminate scents among three groups of individuals: healthy persons and bladder and prostate cancer patients [344]. The evaluation of the experimental data with PCA showed reasonable separation trends. However, a few patients with bladder cancer were included among those with prostate cancer. No healthy person was identified as ill (see Fig. 34).

De Cesare et al. employed a gravimetric EN to detect the "olfactory fingerprints" of microorganisms in soil, with potential relevance for agriculture [340]. The eight Q-TSMRs of the nose were coated with organic polymers and exposed to the headspace atmosphere generated by the investigated samples. Discrimination of PCA scores between non-inoculated and bacterially inoculated soils was possible. Using PLSR, the authors could predict, with reasonable accuracy, the number of inoculated microorganisms.

Metallic vapor Industrial emissions can release metallic pollutants into the environment, often directly into the atmosphere, as metallic vapor. For example, Kabir et al. measured the concentration of metallic quicksilver vapor at the ppbv level (1.4 ppbv LDL) with a SAW transducer covered with a Au sensing layer and having Ni electrodes (to avoid amalgam formation) [508]. Instead of gold as sensing material, silver has also been used [509]. Sabri et al. created Au nanoparticles on the electrodes of a Q-TSMR to detect $\mathrm{Hg}$ vapor with 2.4 ppbv LDL [510].

When considering the achievements addressed in this section reflecting the results reported by several authors, one has to keep in mind that these successes have been obtained in investigations carried out under very controlled conditions, in a friendly laboratory environment, and many more real-world studies will be required before the applications can be widely used.

\section{Achievements and perspectives on gravimetric gas sensors}

The first conclusive accounts below refer to topics contained in this part of the review and are followed by statements emerging from the whole review. They attempt to establish the degree to which the expectations in the field of GGSs have been met and to understand why the advances did not mirror the prospects.

\section{Efforts to improve gravimetric gas sensor performance and their practical implementation}

Because of their simple transduction, appropriate for all recognition processes resulting in receptor mass change, GGSs have been and remain a very attractive choice for applications. As the whole section "Increasing the specificity of the receptors" shows, many attempts have been made to increase the strength and specificity of the analyte-receptor interaction through several types of approaches:

- increasing the complementarity of the receptor towards a given analyte by molecular tailoring, molecular imprinting, use of molecular symmetry (the chiral one, for example), use of cavitands and/or functionalized compounds, use of materials with an enhanced degree of thermodynamic non-ideality (composite, polymorphs, ionic fluids)

- by means of GGS arrays or systems with improved selectivity

- employing adequate sensor data processing at the hardware (analogic) level, or through online/offline dedicated software, based on chemometric methods (PCA, CA, DA, PLS-DA, PLSR) or artificial intelligence

- developing evaluation approaches for specific devices/ applications.

The methods used to coat the MSTs with the sensing layers, chosen to be compatible with the required structure/morphology of the material, are very diverse, ranging from simple (like drop casting, spray coating) to complicated (like atomic layer deposition, biosynthetic methods), sometimes including compound preparation stages (for instance, electro-polymerization). The devised devices have been utilized for the detection of different classes of gases/vapor (flammable/toxic hydrocarbons, inorganic gases, CWAs, humidity, metal vapor), in some cases in the context of well-defined applications: freshness and quality of food, identification of human diseases and personal safety in industrial activity. However, the characterization of the reported devices or applications is not exhaustive or adequately relevant. Several researchers do not thoroughly use their experimental data to infer the sensor parameters (this can be seen in Annex, which encompasses many parameters evaluated by the authors of the present review from data already existing in the published reports). Moreover, the chemometric methods are employed to a limited extent, even for sensor arrays, where they would 
Fig. 31 PCA score clustering for stored fruits according to storage time. Evaluation made with GGS array based on poly(pyrrole) and $\mathrm{TiO}_{2}$ composite. Reproduced with the kind permission of Elsevier B.V. from references [480]
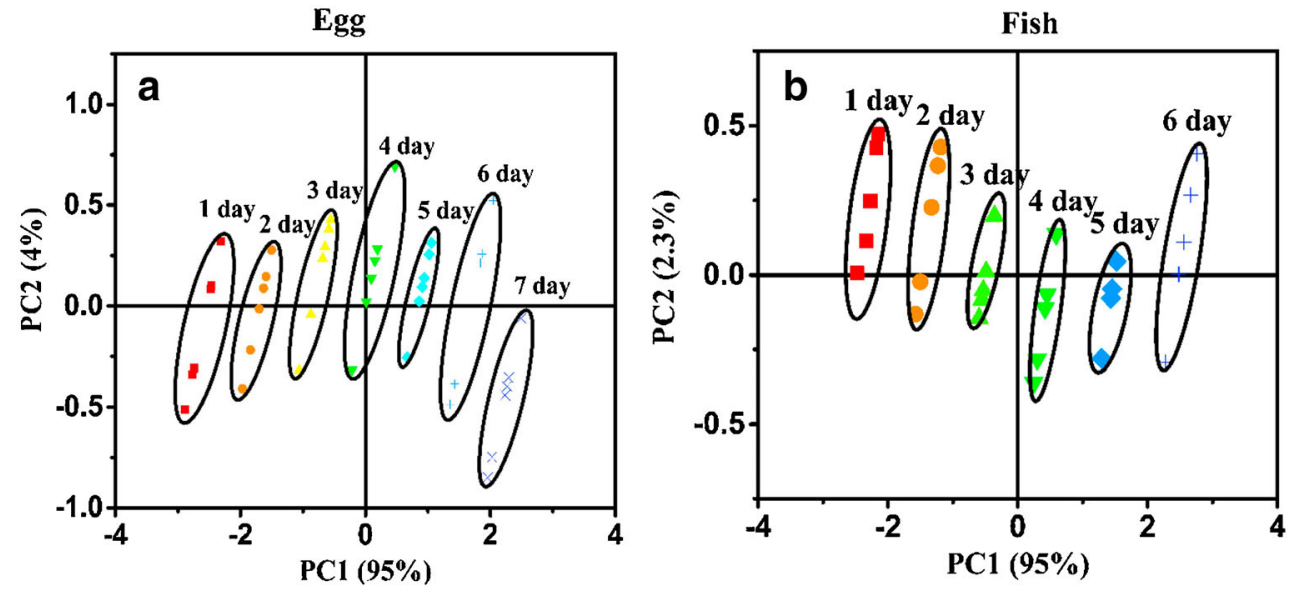

contribute to an increase in the sensor system specificity and accuracy.

\section{The actual development stage of gravimetric gas sensors: expectations, achievements and disappointments}

Despite good scientific understanding and technological progress, the expected breakthrough of GGSs has occurred to a very limited extent in the application field and is almost invisible on the market. Even after increasing the effort spent for devising adsorption-based devices, the market situation did not improve. Some possible reasons are:

a. the mismatch between the operational parameters required by the industrial/customer applications and the "offer" coming from the prototype GGSs. b. the large market request for the detection of certain gases (toxic and flammable/explosive ones) for which the sensing capability and mainly the specificity of GGSs is limited. The optimistic picture of GGSs selectivity in the literature is often misleading, because the cross-sensitivity tests, though performed at relatively high concentrations with respect to that of the target analyte, are not as large as TLV-TWA levels which might actually be encountered in the atmosphere. The most critical is the case of humidity, where the usual environmental level is about 10,000 ppmv.

c. the questionable reliability, mainly due to the soft organic material employed for the receptor manufacture, but also to the mechanical component of the transducer operation (resonant ultra-acoustic vibration). The changes in the sensing layer stiffness, occurring together with changes in the mass, might also alter accurate responses.

d. the sensor price, largely decided by the price of MTS (often using single crystals) is usually higher than the
Fig. 32 The biplot of the scores and loadings in the first two PCs resulting from the responses of a four-sensor SA when exposed to headspace samples of fruit juices. The table in the right panel indicates the average composition of these commercial beverages. Reproduced with the kind permission of Frontiers Media SA from reference [249]

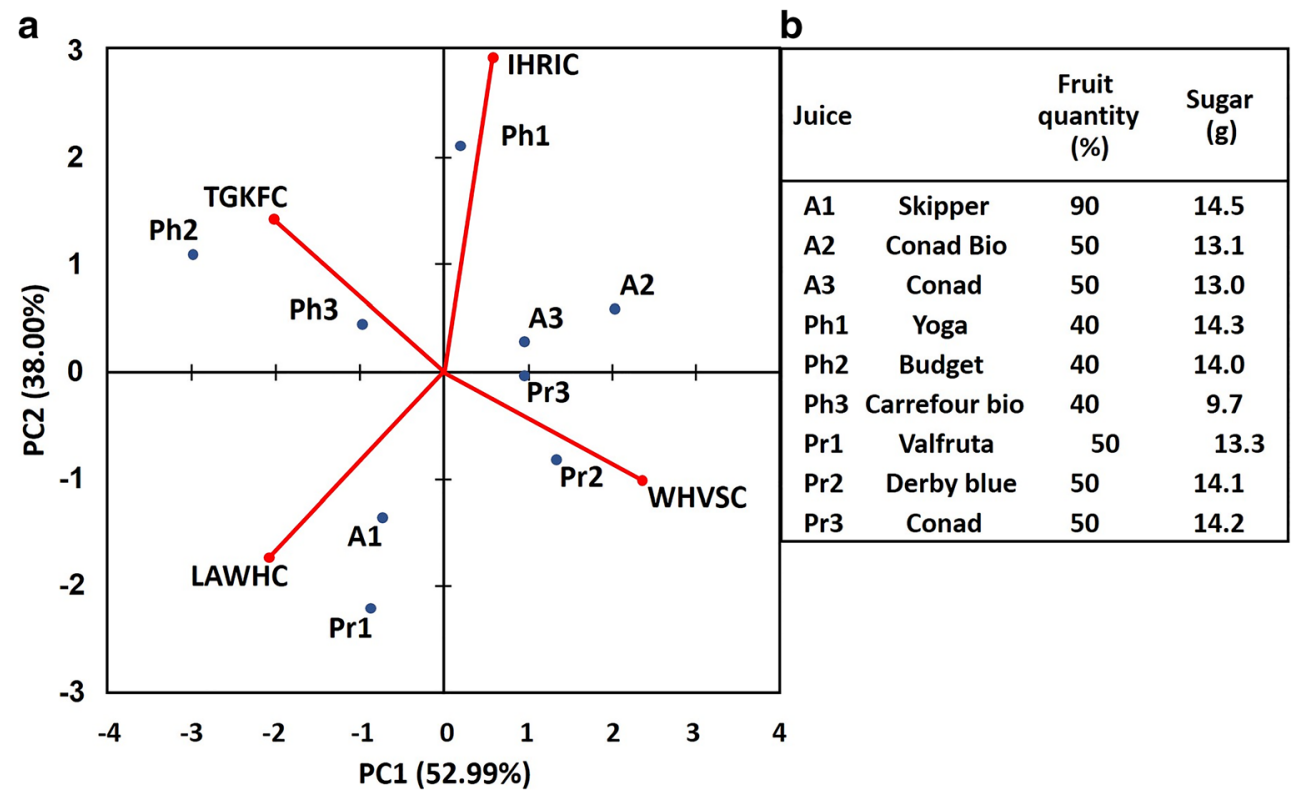


Fig. 33 The response of a differential Q-TSMR sensor system for ammonia detection in large humidity background (refer to the text). The comparison with the exhaled ammonia in human breath demonstrates the potential of the approach. Reproduced with the kind permission of Elsevier B.V. from reference [119]

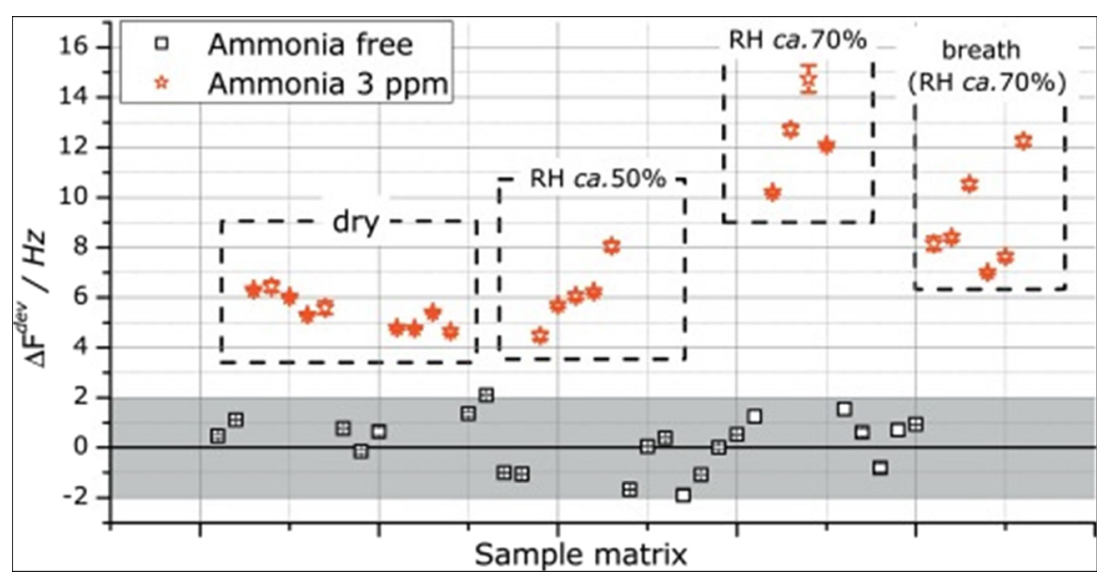

one of some competing categories of devices such as SMOX chemoresitive or polymer capacitive gas sensors.

e. the accuracy of the GGSs is below the one offered by the electrochemical or optical/spectral sensors, which are superior even if more expensive and bulkier.

f. the readout is still more complicated than in the case of the other sensors.

\section{Final remarks}

The investigation on gas sensing devices, particularly gravimetric gas sensors, is continuously growing in importance,

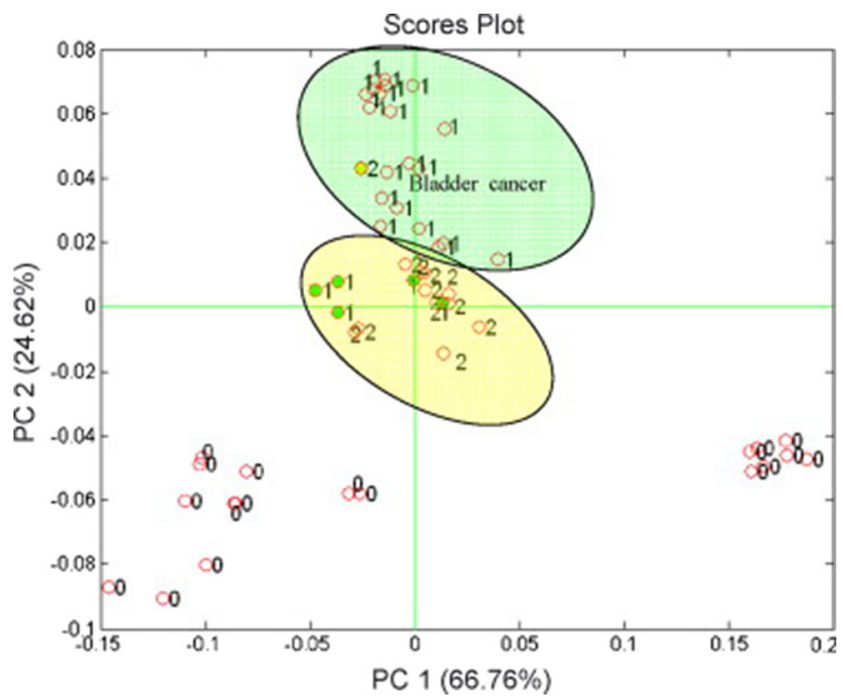

Fig. 34 The PCA score plots with spontaneous clustering of urine analysis results. The event scores are displayed in the first two PCs as red open circles. Events numbered " 0 " and without cluster structure belong to healthy persons. The events numbered "1" and "2" indicate patients having bladder (green cluster) and prostate (yellow cluster) cancer, respectively. Some bladder cancer events are spread among the prostate ones. They are displayed as green filled circles with red contour. Reproduced with the kind permission of Elsevier B.V. from reference [344] volume and geographic extent. The considerable interest in this scientific and applicative topic, witnessed by the increasing number of published reports, publications and patents, reflects the increasing market needs but also the limited success of the previous research approaches in the field. Here, one has to acknowledge the still unripe stage, at least from a market point of view, of gravimetric gas sensors, even after decades of investigations leading to pertinent scientific knowledge. This uncommon situation has, nevertheless, a positive side. It shows that many opportunities are still open, and innovative solutions for gravimetric gas sensors are needed and anticipated.

Funding information Open Access funding provided by Projekt DEAL.

\section{Compliance with ethical standards}

Conflict of interest The authors declare that they have no competing interests.

Research involving humans and/or animals The article is a review and involved no humans or animals in investigations performed by the authors themselves.

Open Access This article is licensed under a Creative Commons Attribution 4.0 International License, which permits use, sharing, adaptation, distribution and reproduction in any medium or format, as long as you give appropriate credit to the original author(s) and the source, provide a link to the Creative Commons licence, and indicate if changes were made. The images or other third party material in this article are included in the article's Creative Commons licence, unless indicated otherwise in a credit line to the material. If material is not included in the article's Creative Commons licence and your intended use is not permitted by statutory regulation or exceeds the permitted use, you will need to obtain permission directly from the copyright holder. To view a copy of this licence, visit http://creativecommons.org/licenses/by/4.0/. 


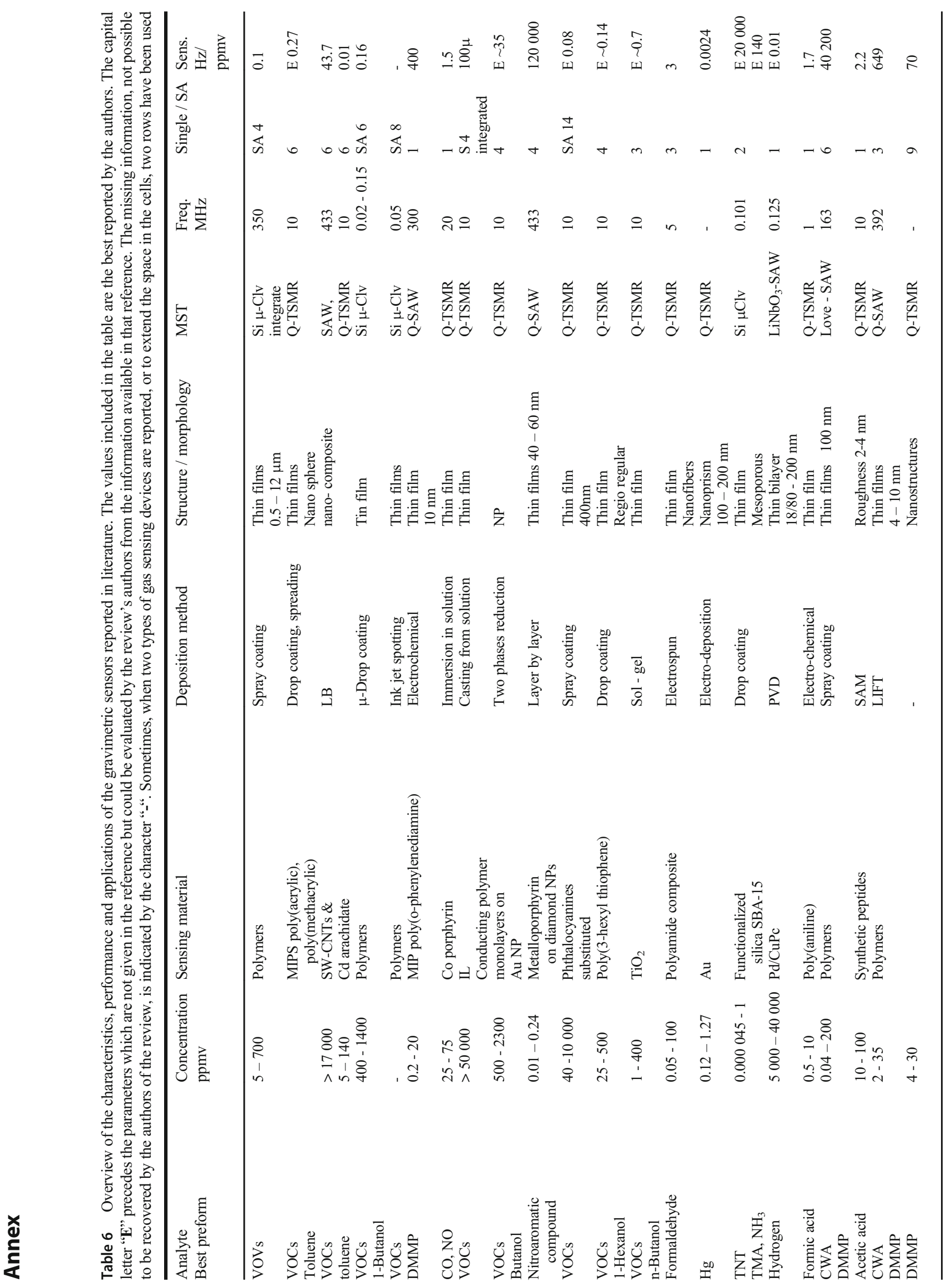




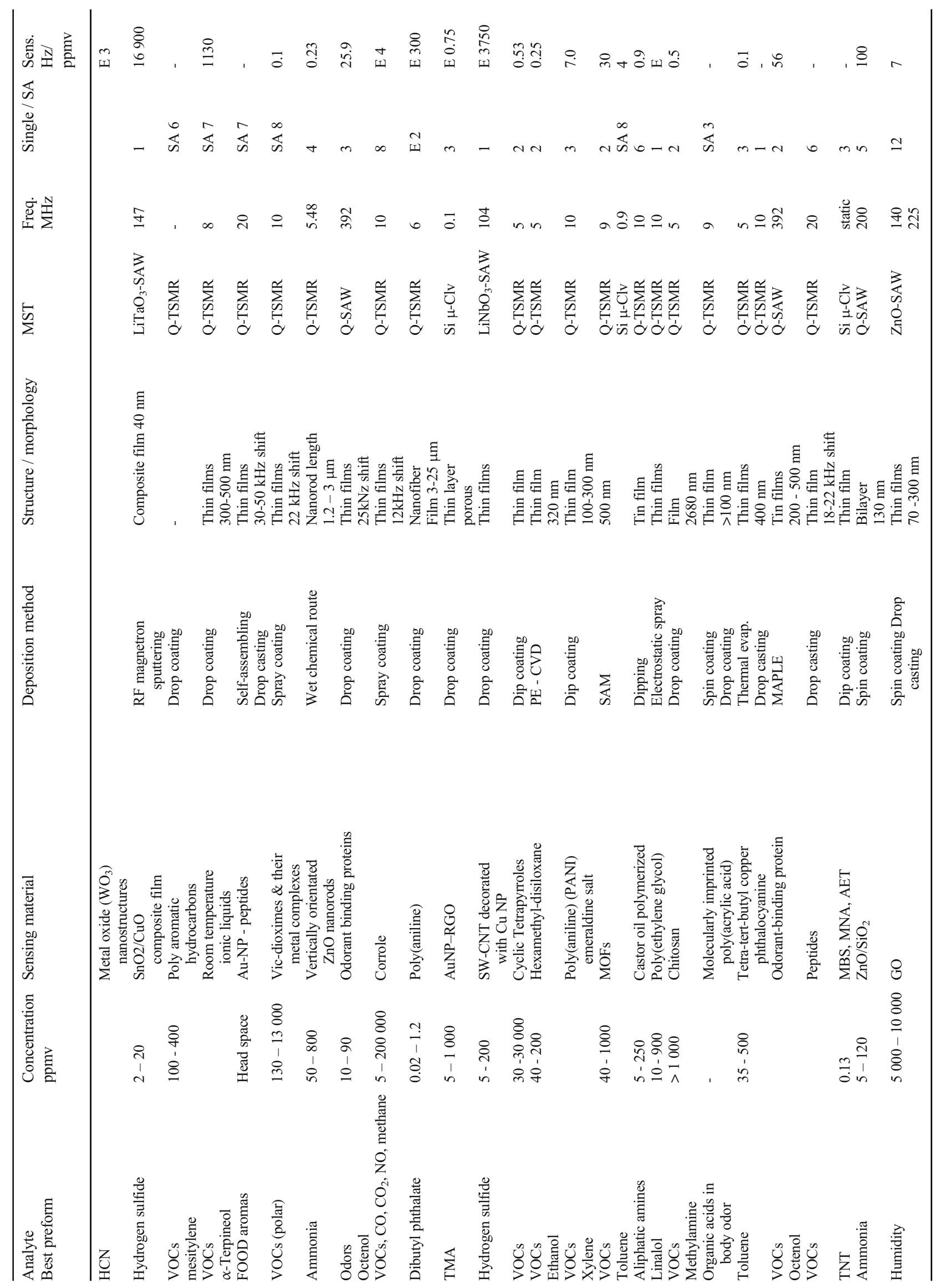




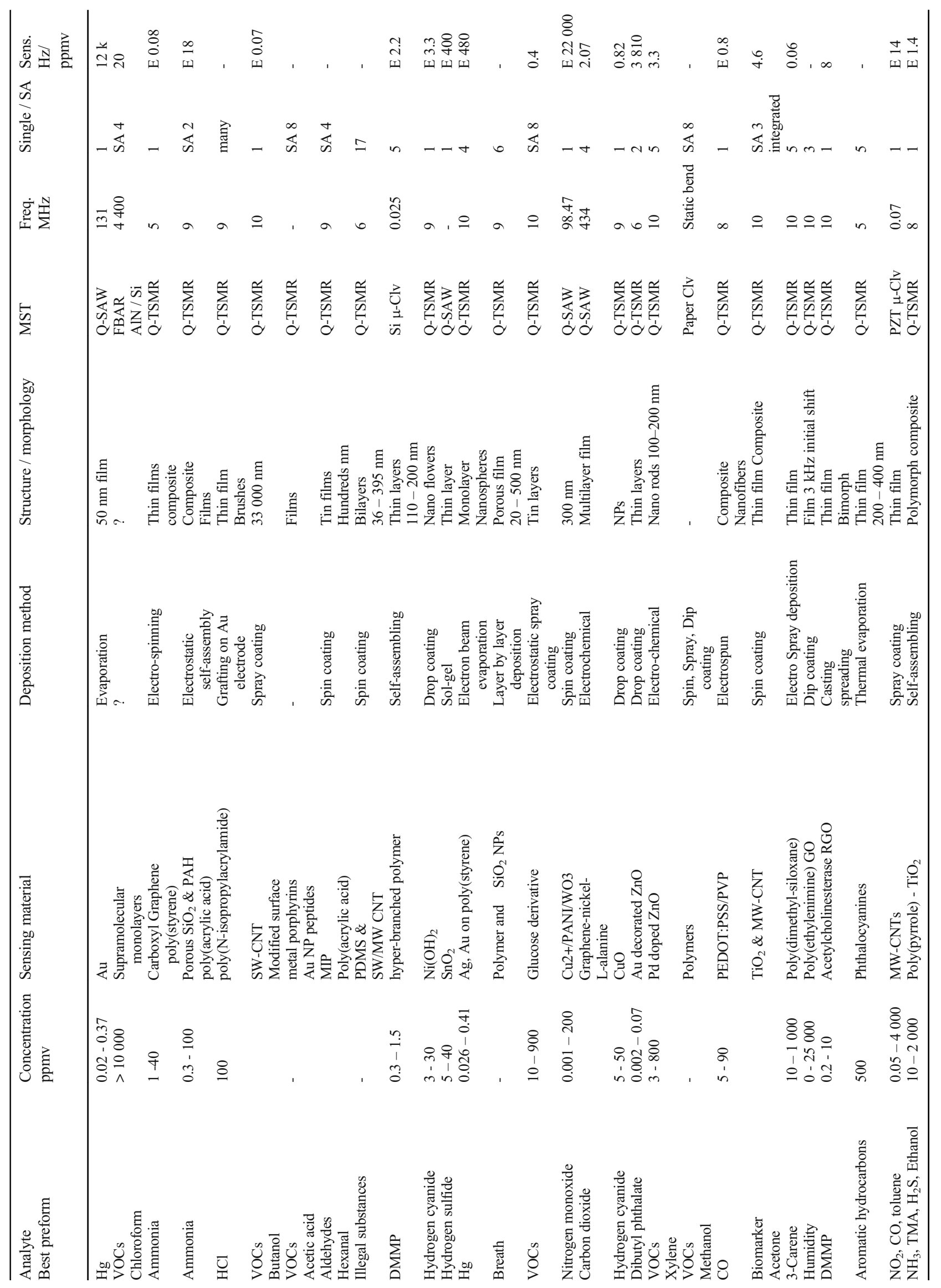




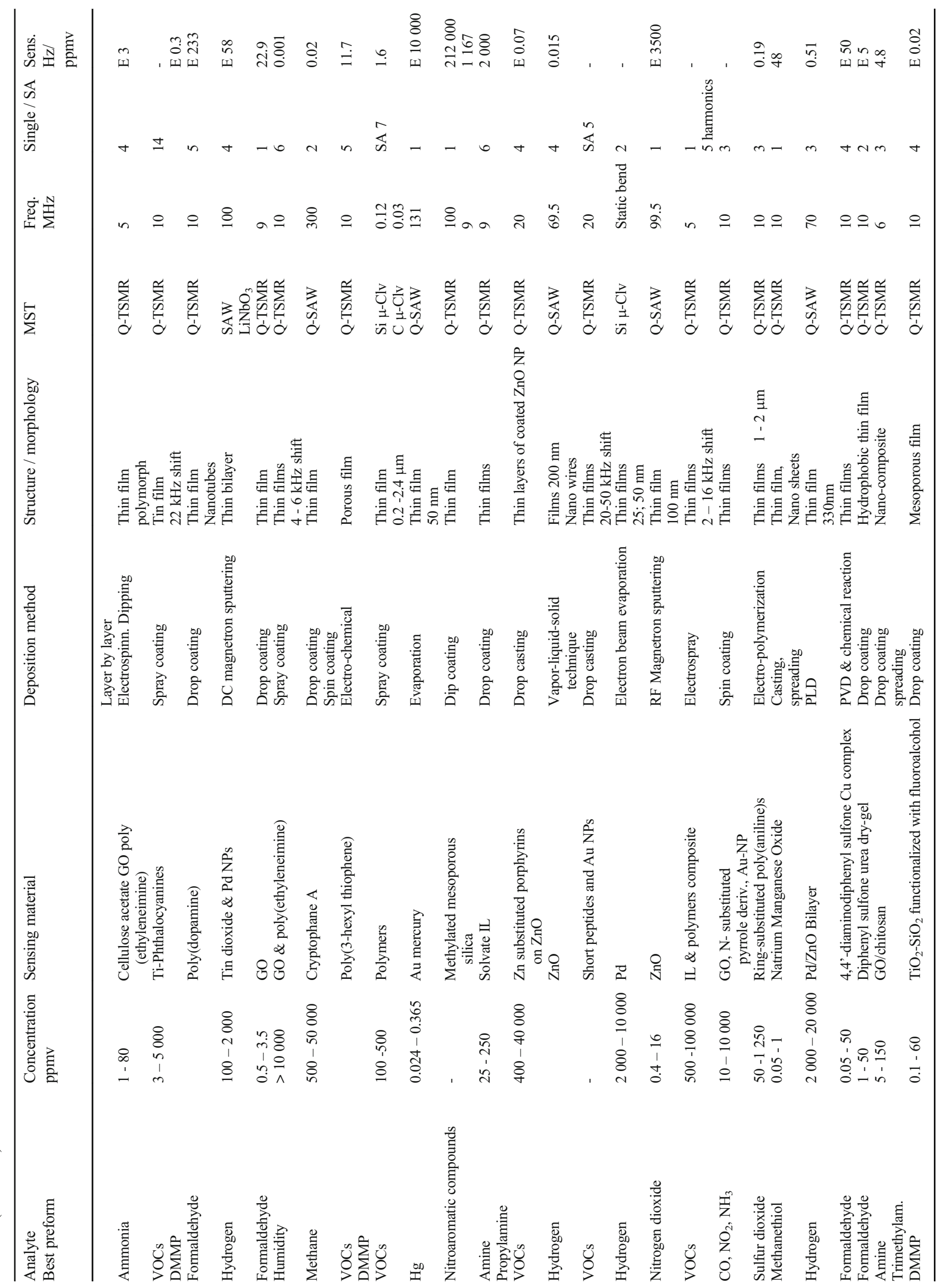




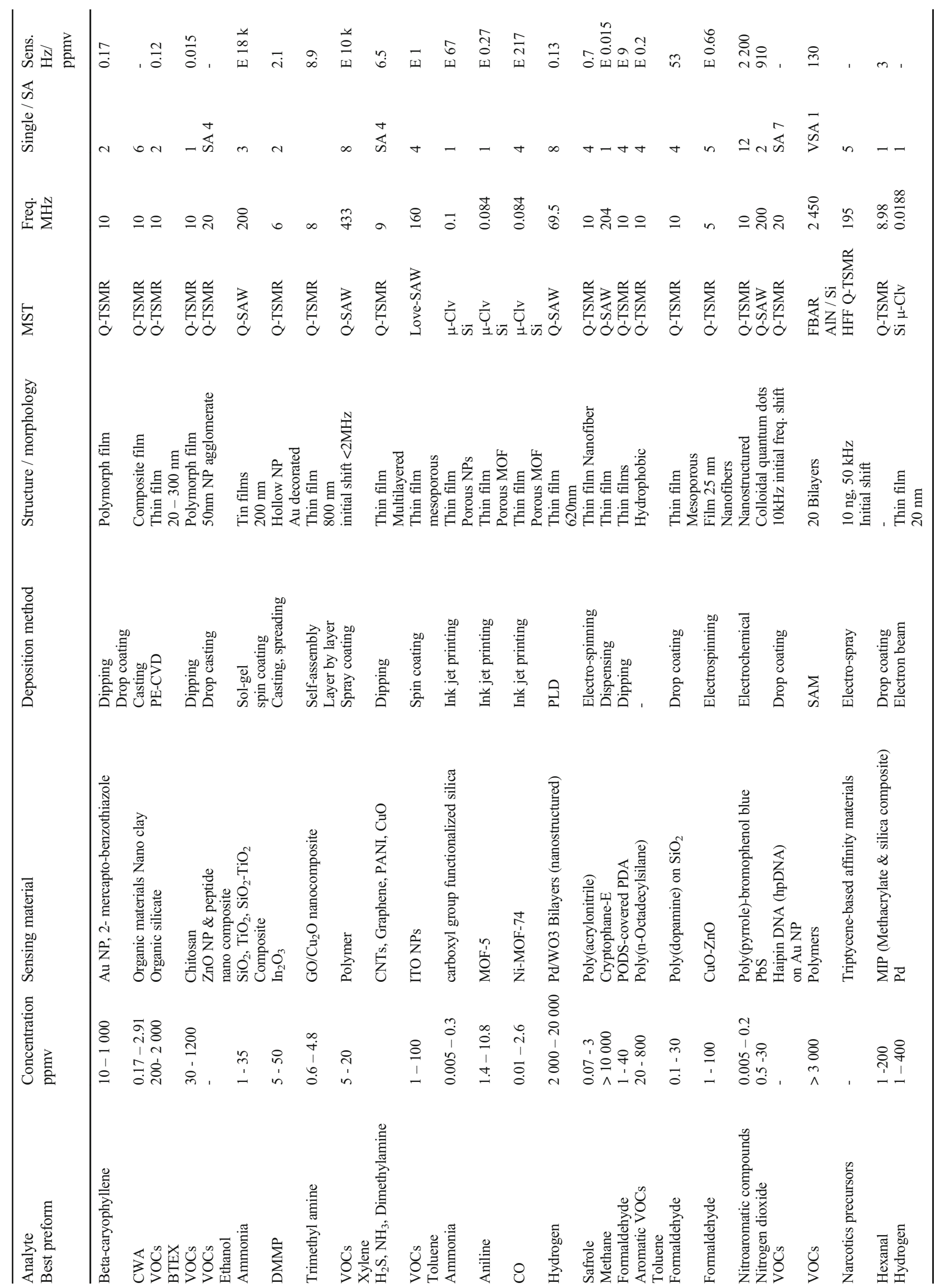




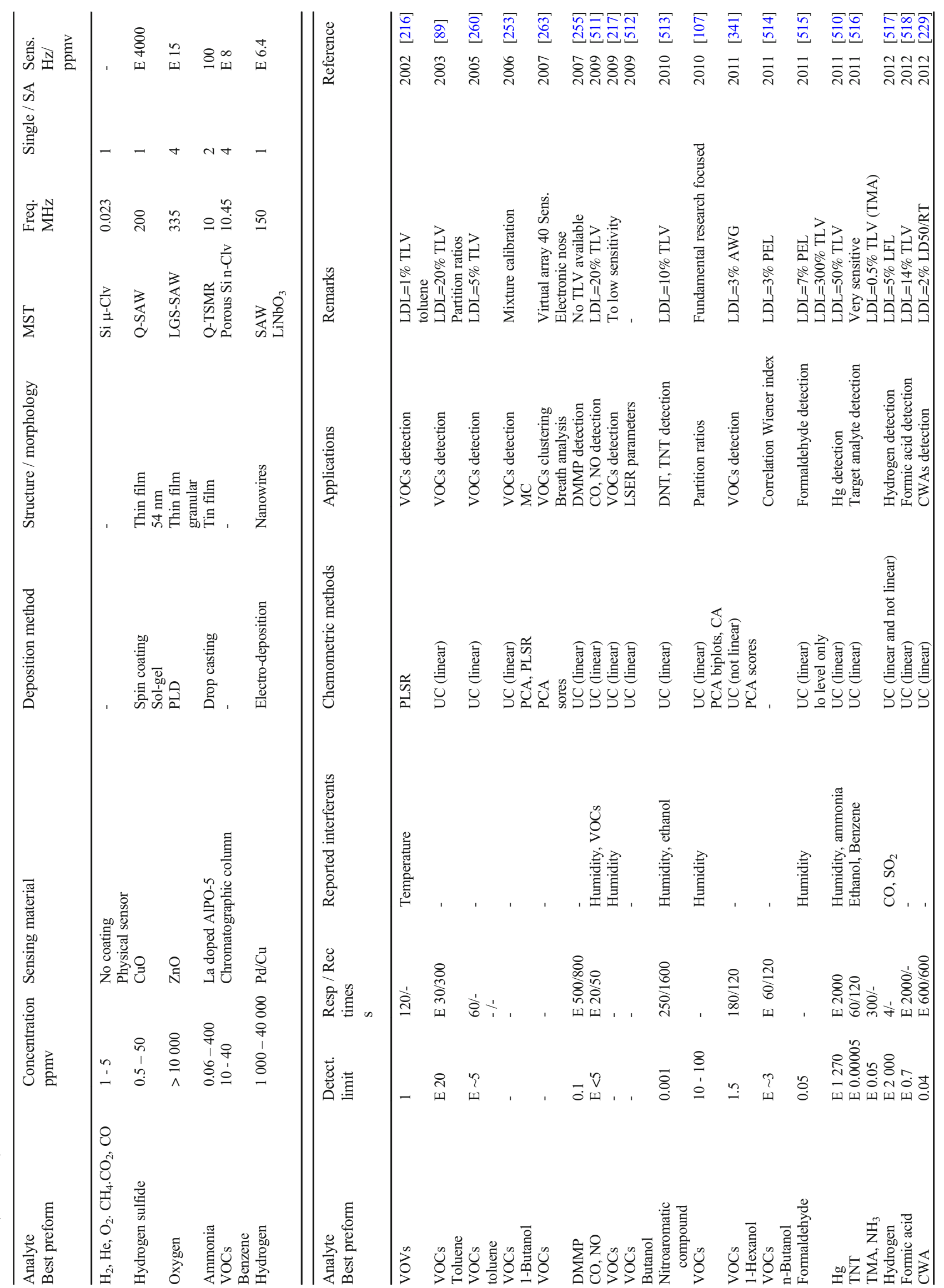




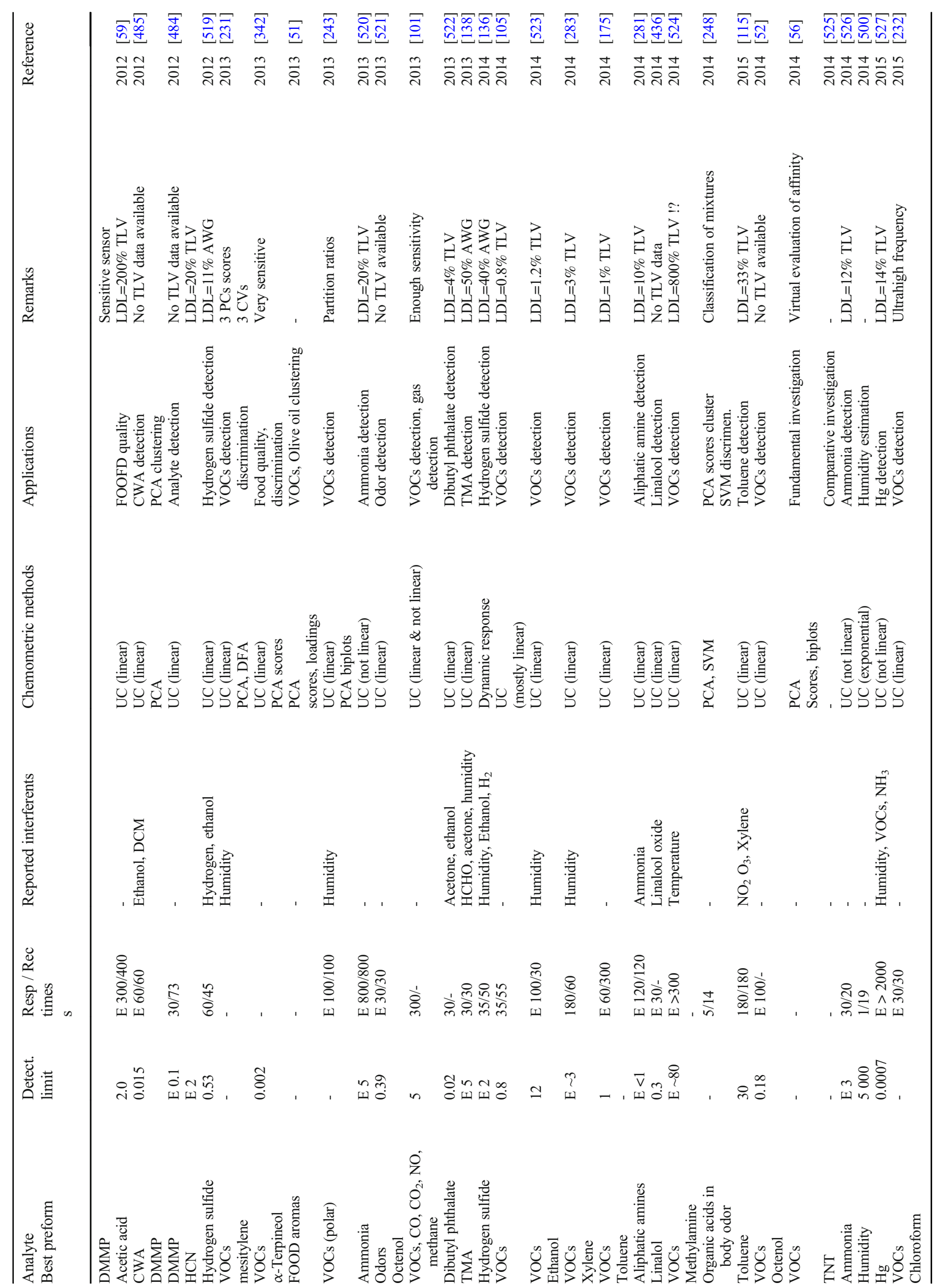




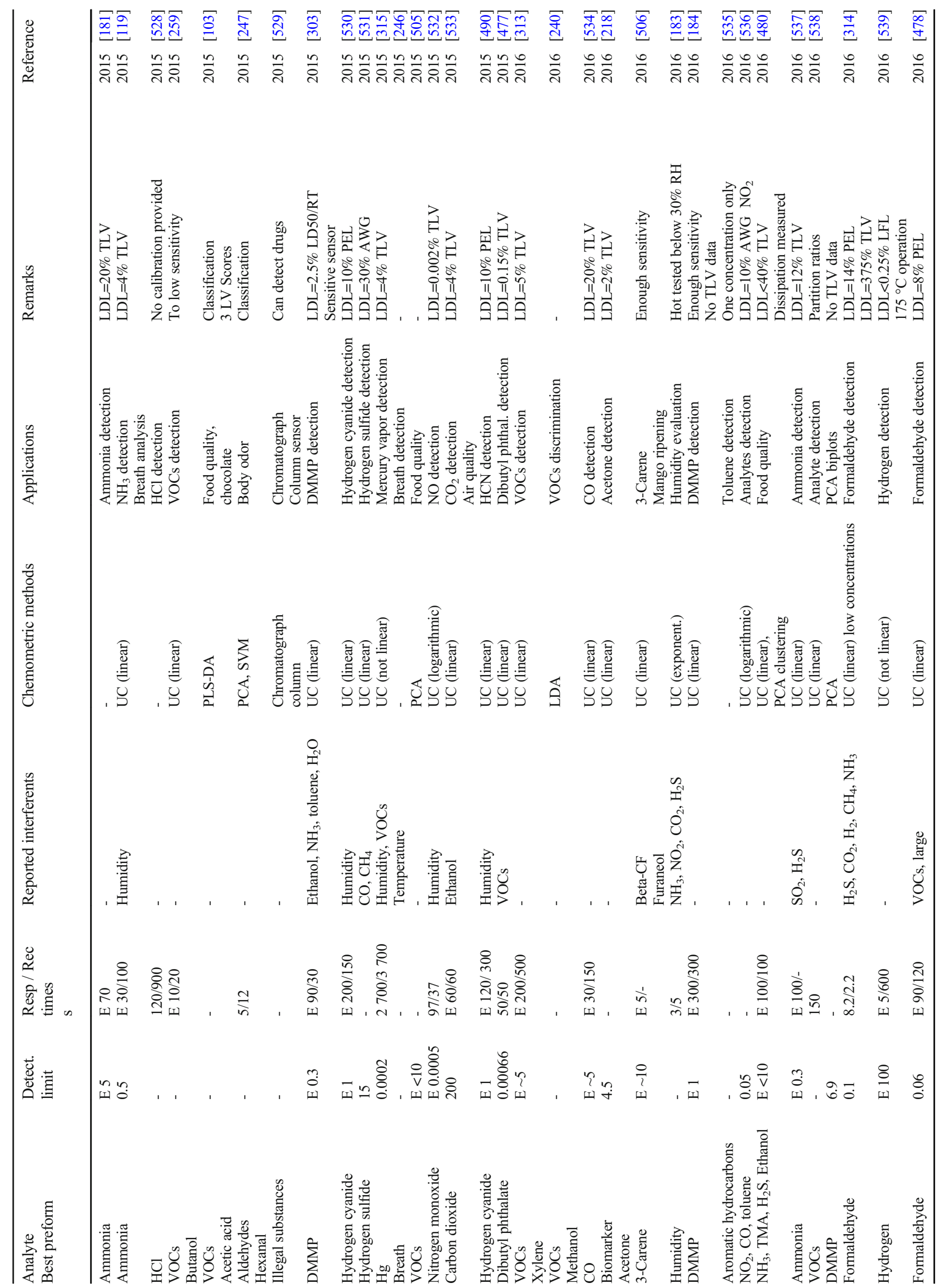




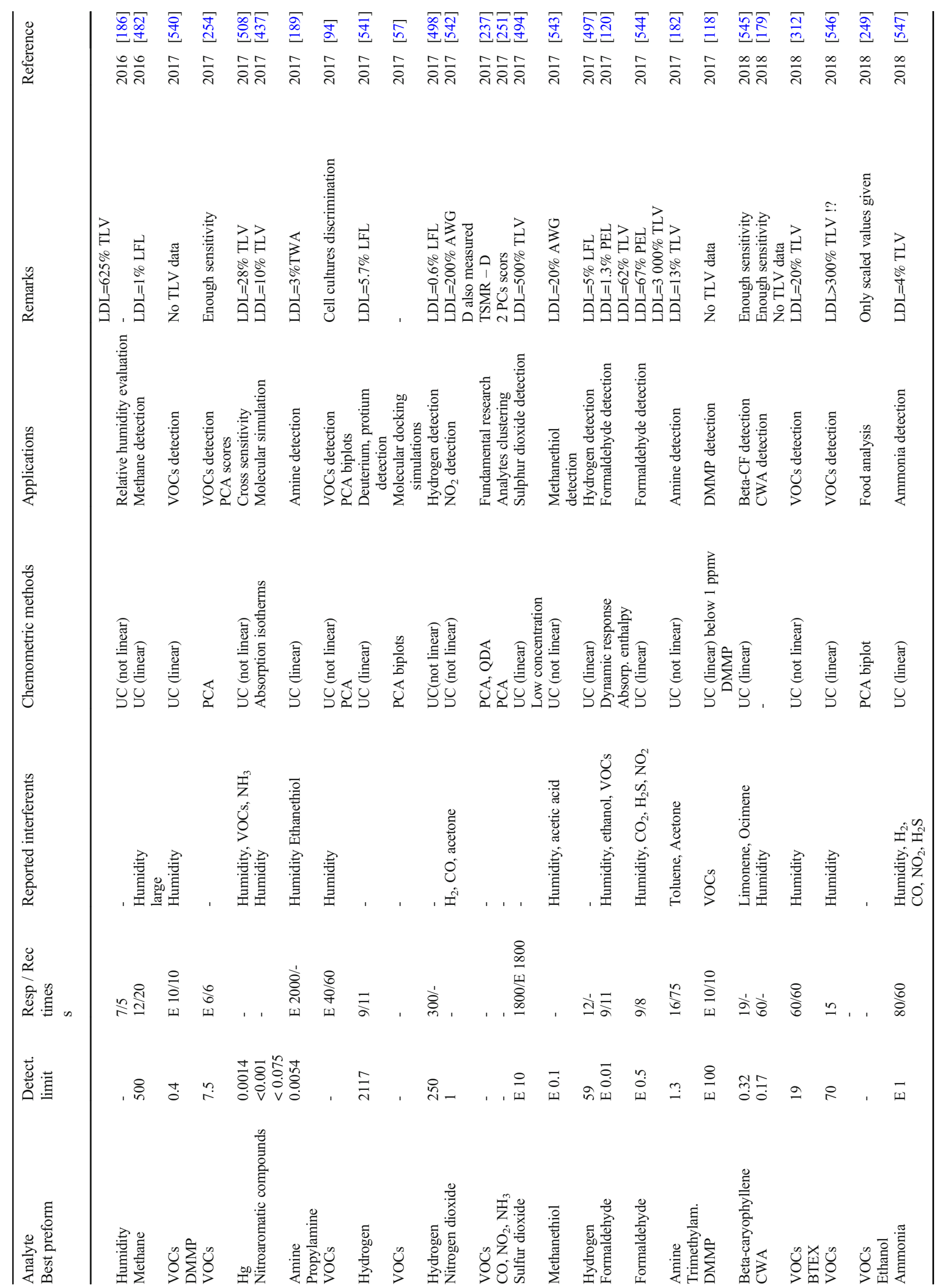




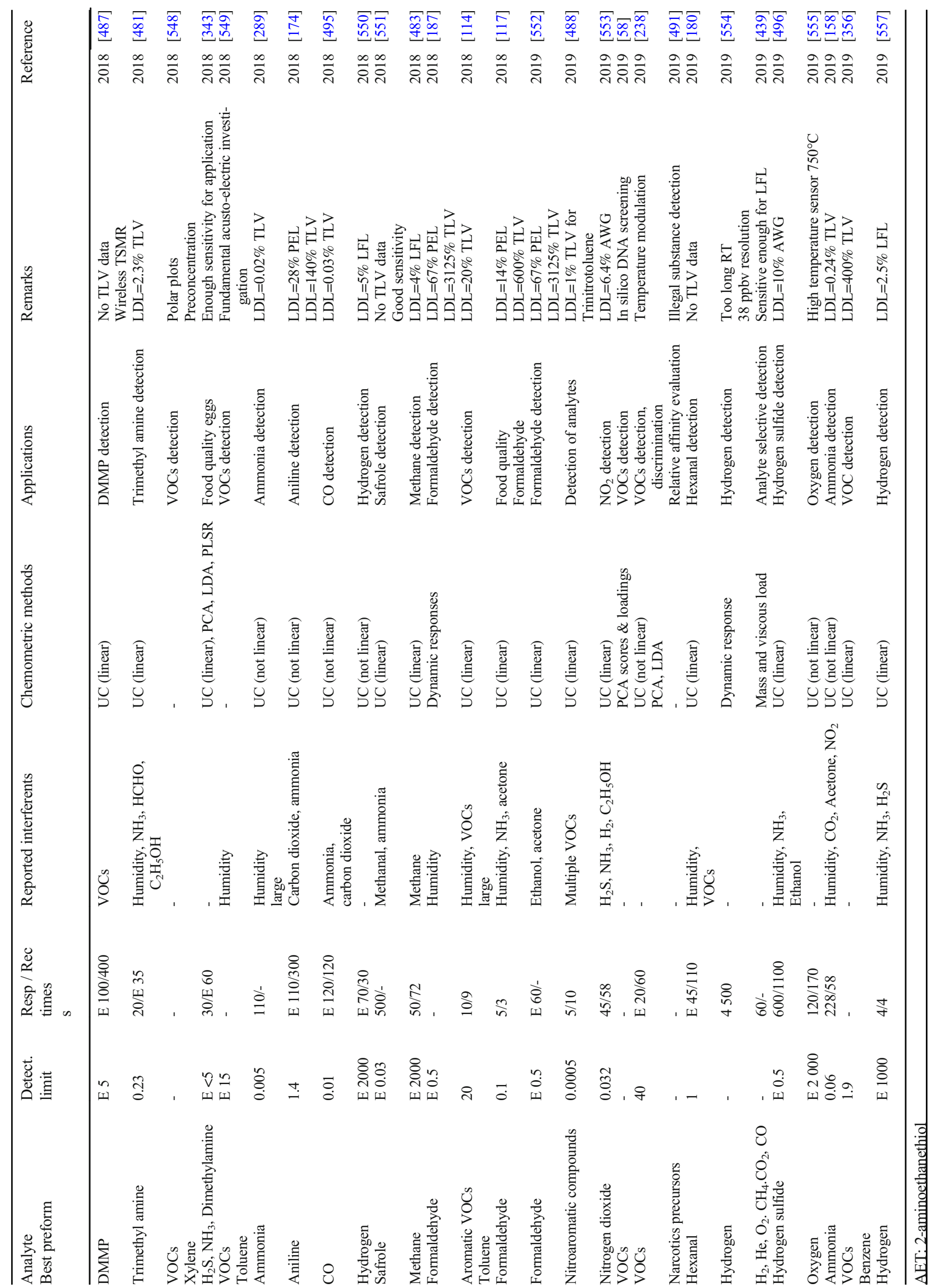




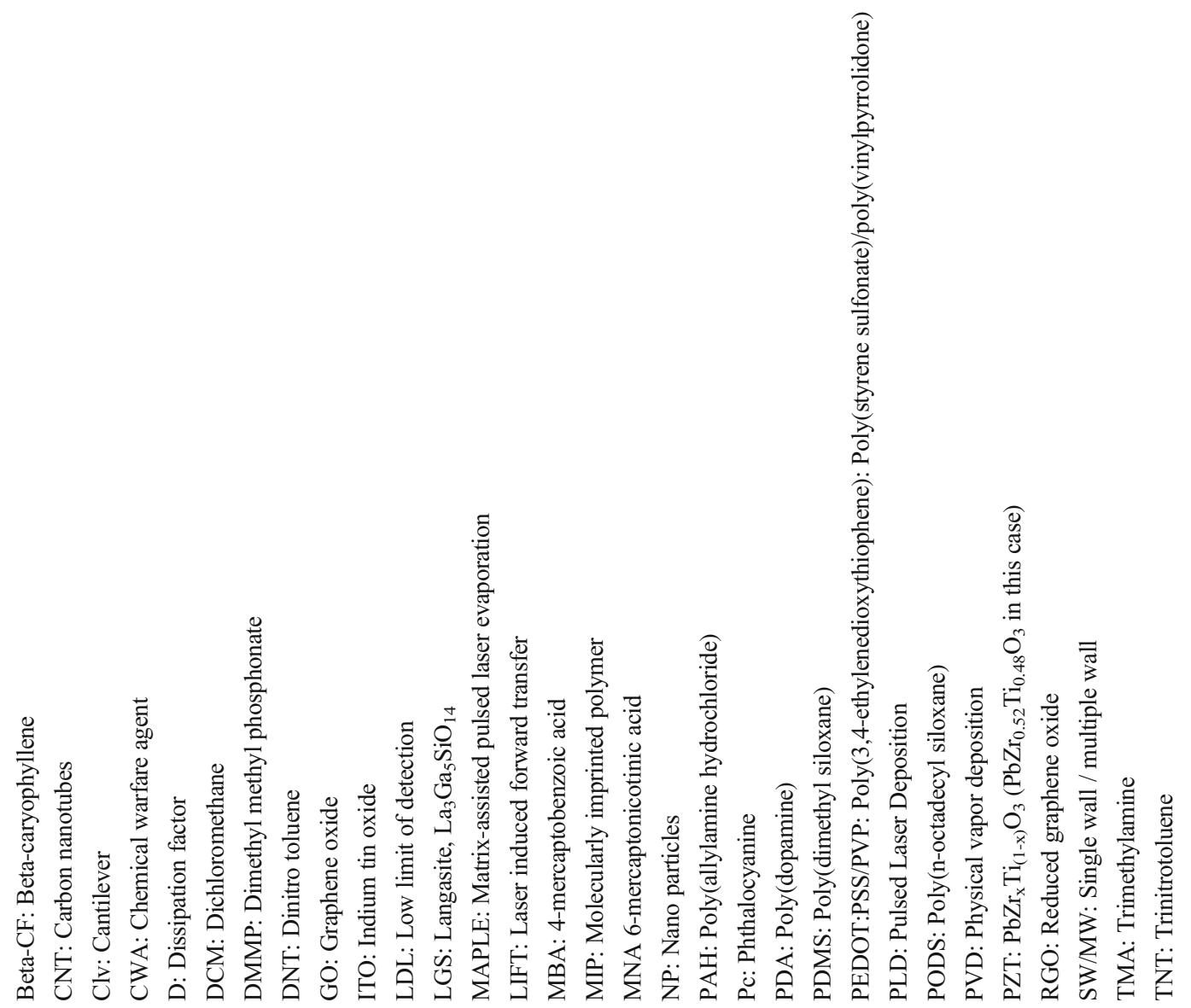




\section{References}

1. Oprea A, Weimar U. Gas sensors based on mass-sensitive transducers part 1: transducers and receptors - basic understanding. Anal Bioanal Chem. 2019 Mar 14;411(9):1761-87. http://link. springer.com/10.1007/s00216-019-01630-7.

2. Hierlemann A, Ricco AJ, Bodenhöfer K, Göpel W. Effective use of molecular recognition in gas sensing: results from acoustic wave and in situ FT-IR measurements. Anal Chem. 1999;71(15):3022-35. https://doi.org/10.1021/ac981311j\% 5Cnhttp://pubs.acs.org/doi/full/10.1021/ac981311j\%5Cnhttp:// pubs.acs.org/doi/pdfplus/10.1021/ac981311j.

3. Fietzek C, Bodenhöfer K, Haisch P, Hees M, Hanack M, Steinbrecher S, et al. Soluble phthalocyanines as coatings for quartz-microbalances: specific and unspecific sorption of volatile organic compounds. Sensors Actuators B Chem. 1999 Sep;57(13):88-98. https://linkinghub.elsevier.com/retrieve/pii/ S0925400599001677.

4. Kamlet MJ, Abboud JLM, Abraham MH, Taft RW. Linear solvation energy relationships. 23. A comprehensive collection of the solvatochromic parameters,.pi.*, $\alpha$, and $\beta$, and some methods for simplifying the generalized solvatochromic equation. J Org Chem. 1983 Aug;48(17):2877-87. http://pubs.acs.org/doi/abs/10.1021/ jo00165a018.

5. Grate JW, Abraham MH. Solubility interactions and the design of chemically selective sorbent coatings for chemical sensors and arrays. Sensors Actuators B Chem. 1991 Feb;3(2):85-111. http://linkinghub.elsevier.com/retrieve/pii/092540059180202U.

6. Behr JP. The lock-and-key principle: the state of the art-100 years on. In: Behr J-P, editor. 2nd ed. Chichester, New York, Brisbane, Toronto, Singapore: John Wiley and Sons; 2008. (Perspectives in supramolecular chemistry). https://books.google.de/books?id= PNdofDBr4XgC.

7. Rudkevich DM. Emerging supramolecular chemistry of gases. Angew Chemie Int Ed. 2004 Jan 23;43(5):558-71. http://doi. wiley.com/10.1002/anie.200300606.

8. Lehn J-M. Supramolecular chemistry - scope and perspectives molecules, supermolecules, and molecular devices (nobel lecture). Angew Chemie Int Ed English. 1988 Jan;27(1):89-112. http://doi. wiley.com/10.1002/anie.198800891.

9. Fischer E. Einfluss der configuration auf die wirkung der enzyme. Berichte der Dtsch Chem Gesellschaft. 1894 Oct;27(3):2985-93. http://doi.wiley.com/10.1002/cber.18940270364.

10. Cram DJ. The design of molecular hosts, guests, and their complexes (nobel lecture). Angew Chemie Int Ed English. 1988 Aug;27(8):1009-20. http://doi.wiley.com/10.1002/anie. 198810093

11. Rebek J. Model studies in molecular recognition. Science (80- ). 1987 Mar 20;235(4795):1478-84. http://www.sciencemag.org/ cgi/doi/10.1126/science.3823899.

12. Schalley CA. Molecular recognition and supramolecular chemistry in the gas phase. Mass Spectrom Rev. 2001;20(5):253-309. http://doi.wiley.com/10.1002/mas.10009.

13. Cain W. To know with the nose: keys to odor identification. Science (80- ). 1979 Feb 2;203(4379):467-70. https://www. sciencemag.org/lookup/doi/10.1126/science.760202.

14. Lancet D. Vertebrate olfactory reception. Annu Rev Neurosci. 1986 Jan 1;9(1):329-55. http://neuro.annualreviews.org/cgi/doi/ 10.1146/annurev.neuro.9.1.329.

15. Firestein S. How the olfactory system makes sense of scents. Nature. 2001 Sep;413(6852):211-8. http://www.nature.com/ articles/35093026.

16. Schaffer ES, Stettler DD, Kato D, Choi GB, Axel R, Abbott LF. Odor perception on the two sides of the brain: consistency despite randomness. Neuron. 2018;98(4):736-742.e3. https://doi.org/10. 1016/j.neuron.2018.04.004.

17. van Hartevelt TJ, Kringelbach ML. The olfactory system. In: The human nervous system: Elsevier; 2012. p. 1219-38. https:// linkinghub.elsevier.com/retrieve/pii/B9780123742360100343.

18. Pelosi P, Pisanelli AM, Baldaccini NE, Gagliardo A. Binding of [ $3 \mathrm{H}]$-2-isobutyl-3-methoxypyrazine to cow olfactory mucosa. Chem Senses. 1981;6(2):77-85. https://academic.oup.com/ chemse/article-lookup/doi/10.1093/chemse/6.2.77.

19. Pelosi P, Baldaccini NE, Pisanelli AM. Identification of a specific olfactory receptor for 2-isobutyl-3-methoxypyrazine. Biochem J. 1982 Jan 1;201(1):245-8. http://www.biochemj.org/cgi/doi/10. 1042/bj2010245.

20. Bignetti E, Cavaggioni A, Pelosi P, Persaud KC, Sorbi RT, Tirindelli R. Purification and characterisation of an odorant-binding protein from cow nasal tissue. Eur J Biochem. 1985 Jun;149(2):227-31. http://doi.wiley.com/10.1111/j.1432-1033. 1985.tb08916.x.

21. Pevsner J, Sklar PB, Snyder SH. Odorant-binding protein: localization to nasal glands and secretions. Proc Natl Acad Sci. $1986 \mathrm{Jul}$ 1;83(13):4942-6. http://www.pnas.org/cgi/doi/10.1073/pnas.83. 13.4942.

22. Pelosi P. Odorant-binding proteins. Crit Rev Biochem Mol Biol. 1994 Jan 26;29(3):199-228. http://www.tandfonline.com/doi/full/ 10.3109/10409239409086801.

23. Flower DR. The lipocalin protein family: structure and function. Biochem J. 1996 Aug 15;318(1):1-14. http://www.biochemj.org/ cgi/doi/10.1042/bj3180001.

24. Pelosi P. Odorant-binding proteins: structural aspects. Ann N Y Acad Sci. 1998 Nov;855(1):281-93. http://doi.wiley.com/10. 1111/j.1749-6632.1998.tb10584.x.

25. Steinbrecht RA. Odorant-binding proteins: expression and function. Ann N Y Acad Sci. 1998 Nov;855(1):323-32. http://doi. wiley.com/10.1111/j.1749-6632.1998.tb10591.x.

26. Tegoni M, Pelosi P, Vincent F, Spinelli S, Campanacci V, Grolli $\mathrm{S}$, et al. Mammalian odorant binding proteins. Biochim Biophys Acta - Protein Struct Mol Enzymol. 2000 Oct;1482(1-2):229-40. https://linkinghub.elsevier.com/retrieve/pii/S0167483800001679.

27. Pelosi P, Mastrogiacomo R, Iovinella I, Tuccori E, Persaud KC. Structure and biotechnological applications of odorant-binding proteins. Appl Microbiol Biotechnol. 2014 Jan 22;98(1):61-70. http://link.springer.com/10.1007/s00253-013-5383-y.

28. Kruse SW, Zhao R, Smith DP, Jones DNM. Structure of a specific alcohol-binding site defined by the odorant binding protein LUSH from Drosophila melanogaster. Nat Struct Mol Biol. 2003 Sep 27;10(9):694-700. http://www.nature.com/articles/nsb960.

29. Leal WS. Odorant reception in insects: roles of receptors, binding proteins, and degrading enzymes. Annu Rev Entomol. 2013 Jan 7;58(1):373-91. http://www.annualreviews.org/doi/10.1146/ annurev-ento-120811-153635.

30. Pelosi P, Iovinella I, Felicioli A, Dani FR. Soluble proteins of chemical communication: an overview across arthropods. Front Physiol. 2014 Aug 27;5(August):1-13. http://journal.frontiersin. org/article/10.3389/fphys.2014.00320/abstract.

31. Menco BP, Cunningham AM, Qasba P, Levy N, Reed RR. Putative odour receptors localize in cilia of olfactory receptor cells in rat and mouse: a freeze-substitution ultrastructural study. J Neurocytol. 1997 Oct;26(10):691-706. http://www.ncbi.nlm.nih. gov/pubmed/9368882.

32. Gaillard I, Rouquier S, Giorgi D. Olfactory receptors. Cell Mol Life Sci. 2004 Feb 1;61(4):456-69. https://linkinghub.elsevier. com/retrieve/pii/B9780123786302006769.

33. de March CA, Kim S-K, Antonczak S, Goddard WA, Golebiowski J. G protein-coupled odorant receptors: From sequence to structure. Protein Sci. 2015 Sep;24(9):1543-8. http:// doi.wiley.com/10.1002/pro.2717. 
34. Buck L, Axel R. A novel multigene family may encode odorant receptors: A molecular basis for odor recognition. Cell. 1991 Apr;65(1):175-87. https://linkinghub.elsevier.com/retrieve/pii/ 009286749190418X.

35. Buck LB. Olfactory receptors and odor coding in mammals. Nutr Rev. 2004 Nov;62(11):S184-8. https://academic.oup.com/ nutritionreviews/article-lookup/doi/10.1111/j.1753-4887.2004. tb00097.x.

36. Malnic B, Hirono J, Sato T, Buck LB. Combinatorial receptor codes for odors. Cell. 1999 Mar;96(5):713-23. https:// linkinghub.elsevier.com/retrieve/pii/S0092867400805814.

37. Buck LB. Unraveling the sense of smell. NobelPrize.org. Nobel Media AB 2019. 2004 [cited 2019 Aug 20]. https://www. nobelprize.org/prizes/medicine/2004/buck/lecture/\%3E.

38. Axel R. The molecular logic of smell. Sci Am. 1995 Oct;273(4): 154-9. http://www.nature.com/doifinder/10.1038/ scientificamerican1095-154.

39. Hou Y, Jaffrezic-Renault N, Martelet C, Zhang A, Minic-Vidic J, Gorojankina T, et al. A novel detection strategy for odorant molecules based on controlled bioengineering of rat olfactory receptor I7. Biosens Bioelectron. 2007 Feb;22(7):1550-5. https:// linkinghub.elsevier.com/retrieve/pii/S0956566306002971.

40. Sato K, Takeuchi S. Chemical vapor detection using a reconstituted insect olfactory receptor complex. Angew Chemie Int Ed. 2014 Oct 27;53(44):11798-802. http://doi.wiley.com/10. 1002/anie.201404720.

41. Capo A, Pennacchio A, Varriale A, D'Auria S, Staiano M. The porcine odorant-binding protein as molecular probe for benzene detection. Zhou Z, editor. PLoS One. 2018 Sep 5;13(9):e0202630. https://doi.org/10.1371/journal.pone.0202630.

42. Cave JW, Wickiser JK, Mitropoulos AN. Progress in the development of olfactory-based bioelectronic chemosensors. Biosens Bioelectron. 2019 Jan;123(July 2018):211-22. https:// linkinghub.elsevier.com/retrieve/pii/S0956566318306705.

43. Persaud KC. Electronic gas and odour detectors that mimic chemoreception in animals. TrAC Trends Anal Chem. 1992 Feb;11(2):61-7. https://linkinghub.elsevier.com/retrieve/pii/ 016599369280079L.

44. Sankaran S, Khot LR, Panigrahi S. Biology and applications of olfactory sensing system: A review. Sensors Actuators, B Chem. 2012;171-172:1-17. https://doi.org/10.1016/j.snb.2012.03.029.

45. Barbosa AJM, Oliveira AR, Roque ACA. Protein- and peptidebased biosensors in artificial olfaction. Trends Biotechnol. 2018 Dec;36(12):1244-58. https://linkinghub.elsevier.com/retrieve/pii/ S0167779918301951.

46. Wasilewski T, Gębicki J, Kamysz W. Advances in olfaction-inspired biomaterials applied to bioelectronic noses. Sensors Actuators B Chem. 2018 Mar;257:511-37. https://linkinghub. elsevier.com/retrieve/pii/S0925400517319871.

47. Alfinito E, Millithaler JF, Pennetta C, Reggiani L. A single protein based nanobiosensor for odorant recognition. Microelectronics J. 2010;41(11):718-22. https://doi.org/10.1016/j.mejo.2010.07.006.

48. Capone S, De Pascali C, Francioso L, Siciliano P, Persaud KC, Pisanelli AM. Odorant binding proteins as sensing layers for novel gas biosensors: an impedance spectroscopy characterization. In. 2011:317-24. http://link.springer.com/10.1007/978-94-0071324-6 49.

49. Goldsmith BR, Mitala JJ, Josue J, Castro A, Lerner MB, Bayburt $\mathrm{TH}$, et al. Biomimetic chemical sensors using nanoelectronic readout of olfactory receptor proteins. ACS Nano. $2011 \mathrm{Jul}$ 26;5(7): 5408-16. https://pubs.acs.org/doi/10.1021/nn200489j.

50. Kotlowski C, Larisika M, Guerin PM, Kleber C, Kröber T, Mastrogiacomo R, et al. Fine discrimination of volatile compounds by graphene-immobilized odorant-binding proteins. Sensors Actuators B Chem. 2018 Mar;256:564-72. https:// linkinghub.elsevier.com/retrieve/pii/S0925400517319950.
51. Compagnone D, Fusella GC, Del Carlo M, Pittia P, Martinelli E, Tortora L, et al. Gold nanoparticles-peptide based gas sensor arrays for the detection of food aromas. Biosens Bioelectron. 2013;42(1):618-25. https://doi.org/10.1016/j.bios.2012.10.096.

52. Di Pietrantonio F, Benetti M, Dinca V, Cannatà D, Verona E, D'Auria S, et al. Tailoring odorant-binding protein coatings characteristics for surface acoustic wave biosensor development. Appl Surf Sci. 2014;302:250-5. https://doi.org/10.1016/j.apsusc.2013. 10.112 .

53. ILO, WHO E. ICSC: 0620 / 1996. http://www.ilo.org/dyn/icsc/ showcard.display?p_lang=en\&p_card_id $=0620 \& p \_v e r s i o n=2$.

54. Zhao X, Ashley GM, Garcia-Gancedo L, Jin H, Luo J, Flewitt AJ, et al. Protein functionalized $\mathrm{ZnO}$ thin film bulk acoustic resonator as an odorant biosensor. Sensors Actuators, B Chem. 2012;163(1):242-6. https://doi.org/10.1016/j.snb.2012.01.046.

55. Wu T-Z, Lo Y-R, Chan E-C. Exploring the recognized bio-mimicry materials for gas sensing. Biosens Bioelectron. 2001 Dec;16(9-12):945-53. https://linkinghub.elsevier.com/retrieve/ pii/S0956566301002159.

56. Pizzoni D, Mascini M, Lanzone V, Del Carlo M, Di Natale C, Compagnone D. Selection of peptide ligands for piezoelectric peptide based gas sensors arrays using a virtual screening approach. Biosens Bioelectron. 2014;52:247-54. https://doi.org/10. 1016/j.bios.2013.08.044.

57. Mascini M, Pizzoni D, Perez G, Chiarappa E, Di Natale C, Pittia P, et al. Tailoring gas sensor arrays via the design of short peptides sequences as binding elements. Biosens Bioelectron. 2017 Jul;93(May 2016):161-9. https://linkinghub.elsevier.com/ retrieve/pii/S0956566316309113.

58. Mascini M, Gaggiotti S, Della Pelle F, Wang J, Pingarrón JM, Compagnone D. Hairpin DNA-AuNPs as molecular binding elements for the detection of volatile organic compounds. Biosens Bioelectron. 2019 Jan;123(July 2018):124-30. https://linkinghub. elsevier.com/retrieve/pii/S0956566318305293.

59. Panigrahi S, Sankaran S, Mallik S, Gaddam B, Hanson AA. Olfactory receptor-based polypeptide sensor for acetic acid VOC detection. Mater Sci Eng C. 2012;32(6):1307-13. https://doi.org/ 10.1016/j.msec.2011.11.003.

60. Peer HK. Reviewed: molecularly imprinted polymers: the next generation. Anal Chem. 2003 Sep;75(17):376 A-83 A. https:// pubs.acs.org/doi/10.1021/ac031385h.

61. Yan H, Row K. characteristic and synthetic approach of molecularly imprinted polymer. Int J Mol Sci. 2006 Jun 29;7(5):155-78. http://www.mdpi.com/1422-0067/7/5/155.

62. Polyakov MV. Adsorption properties and structure of silica gel. Zhurnal Fizieskoj Khimii/Akademiya SSSR. 1931;2:799-805.

63. Whitcombe MJ, Kirsch N, Nicholls IA. Molecular imprinting science and technology: a survey of the literature for the years 20042011. J Mol Recognit. 2014 Jun;27(6):297-401. http://doi.wiley. com/10.1002/jmr.2347.

64. Mosbach K. Molecular imprinting. Trends Biochem Sci. 1994 Jan;19(1):9-14. https://linkinghub.elsevier.com/retrieve/pii/ 096800049490166X.

65. Wulff G. Molecular imprinting in cross-linked materials with the aid of molecular templates - a way towards artificial antibodies. Angew Chemie Int Ed English. 1995 Sep 15;34(17):1812-32. http://doi.wiley.com/10.1002/anie.199518121.

66. Dickert FL, Hayden O. Imprinting with sensor development - On the way to synthetic antibodies. Fresenius J Anal Chem. $1999 \mathrm{Jul}$ 16;364(6):506-11. http://link.springer.com/10.1007/ s002160051376.

67. Dickert F. Molecular imprinting in chemical sensing. TrAC Trends Anal Chem. 1999 Mar;18(3):192-9. https://linkinghub. elsevier.com/retrieve/pii/S016599369800123X. 
68. Haupt K, Mosbach K. Molecularly imprinted polymers and their use in biomimetic sensors. Chem Rev. 2000 Jul;100(7):2495-504. https://pubs.acs.org/doi/10.1021/cr990099w.

69. Haupt K. Imprinted polymers - Tailor-made mimics of antibodies and receptors. Chem Commun. 2003 Jan 7;9(2):171-8. http:/ xlink.rsc.org/?DOI=b207596b.

70. Cormack PA, Elorza AZ. Molecularly imprinted polymers: synthesis and characterisation. J Chromatogr B. 2004 May;804(1): 173-82. https://linkinghub.elsevier.com/retrieve/pii/ S1570023204001412.

71. Kandimalla VB, Ju H. Molecular imprinting: a dynamic technique for diverse applications in analytical chemistry. Anal Bioanal Chem. 2004 Oct 8;380(4):587-605. http://link.springer.com/10. 1007/s00216-004-2793-9.

72. Mahony JO, Nolan K, Smyth MR, Mizaikoff B. Molecularly imprinted polymers - potential and challenges in analytical chemistry. Anal Chim Acta. 2005 Apr;534(1):31-9. http://inkinghub. elsevier.com/retrieve/pii/S0003267004008591.

73. Ye L, Mosbach K. Molecular imprinting: synthetic materials as substitutes for biological antibodies and receptors $\uparrow$. Chem Mater. 2008 Feb;20(3):859-68. https://pubs.acs.org/doi/10.1021/ $\mathrm{cm} 703190 \mathrm{w}$.

74. Beltran A, Borrull F, Marcé RM, Cormack PAG. Molecularlyimprinted polymers: useful sorbents for selective extractions. TrAC Trends Anal Chem. 2010 Dec;29(11):1363-75. https:// linkinghub.elsevier.com/retrieve/pii/S0165993610002529.

75. Vasapollo G, Del Sole R, Mergola L, Lazzoi MR, Scardino A, Scorrano S, et al. Molecularly imprinted polymers: present and future prospective. Int J Mol Sci. 2011 Sep 14;12(9):5908-45. http://www.mdpi.com/1422-0067/12/9/5908.

76. Chen L, Xu S, Li J. Recent advances in molecular imprinting technology: current status, challenges and highlighted applications. Chem Soc Rev. 2011;40(5):2922. http://xlink.rsc.org/? DOI $=\mathrm{c} 0 \mathrm{cs} 00084 \mathrm{a}$.

77. Lofgreen JE, Ozin GA. Controlling morphology and porosity to improve performance of molecularly imprinted sol-gel silica. Chem Soc Rev. 2014;43(3):911-33. http://xlink.rsc.org/?DOI= C3CS60276A.

78. Chen L, Wang X, Lu W, Wu X, Li J. Molecular imprinting: perspectives and applications. Chem Soc Rev. 2016;45(8):2137211. http://xlink.rsc.org/?DOI=C6CS00061D.

79. BelBruno JJ. Molecularly Imprinted Polymers. Chem Rev. 2019 Jan 9;119(1):94-119. https://pubs.acs.org/doi/10.1021/acs. chemrev.8b00171

80. Wulff G. Selective binding to polymers via covalent bonds. The construction of chiral cavities as specific receptor sites. Pure Appl Chem. 1982 Jan 1;54(11):2093-102. http://www.degruyter.com/ $\mathrm{view} / \mathrm{j} / \mathrm{pac} .1982 .54$.issue-11/pac $198254112093 /$ pac198254112093.xml.

81. Arshady R, Mosbach K. Synthesis of substrate-selective polymers by host-guest polymerization. Die Makromol Chemie. 1981;182(2):687-92. https://doi.org/10.1002/macp.1981. 021820240

82. Lieberzeit PA, Afzal A, Glanzing G, Dickert FL. Molecularly imprinted sol-gel nanoparticles for mass-sensitive engine oil degradation sensing. Anal Bioanal Chem. 2007 Aug 23;389(2):4416. http://link.springer.com/10.1007/s00216-007-1274-3.

83. Batra D, Shea KJ. Combinatorial methods in molecular imprinting. Curr Opin Chem Biol. 2003 Jun;7(3):434-42. https:// linkinghub.elsevier.com/retrieve/pii/S1367593103000607.

84. Nicholls IA, Adbo K, Andersson HS, Andersson PO, Ankarloo J, Hedin-Dahlström J, et al. Can we rationally design molecularly imprinted polymers? Anal Chim Acta. 2001 May;435(1):9-18. https://linkinghub.elsevier.com/retrieve/pii/S0003267001009321.
85. Kim H, Spivak DA. New insight into modeling non-covalently imprinted polymers. J Am Chem Soc. 2003 Sep;125(37):1126975. https://pubs.acs.org/doi/10.1021/ja0361502.

86. Nicholls IA, Andersson HS, Charlton C, Henschel H, Karlsson BCG, Karlsson JG, et al. Theoretical and computational strategies for rational molecularly imprinted polymer design. Biosens Bioelectron. 2009 Nov;25(3):543-52. https://inkinghub.elsevier. com/retrieve/pii/S0956566309001821.

87. Valenzano L, Civalleri B, Chavan S, Palomino GT, Areán CO, Bordiga S. Computational and experimental studies on the adsorption of $\mathrm{CO}, \mathrm{N} 2$, and $\mathrm{CO} 2$ on Mg-MOF-74. J Phys Chem C. 2010;114(25):11185-91. http://pubs.acs.org/doi/abs/10.1021/ jp102574f.

88. Cowen T, Karim K, Piletsky S. Computational approaches in the design of synthetic receptors - A review. Anal Chim Acta. 2016 Sep;936:62-74. https://linkinghub.elsevier.com/retrieve/pii/ S0003267016308480.

89. Fu Y, Finklea HO. Quartz crystal microbalance sensor for organic vapor detection based on molecularly imprinted polymers. Anal Chem. 2003 Oct;75(20):5387-93. https://pubs.acs.org/doi/10. 1021/ac034523b.

90. Bunte G, Hürttlen J, Pontius H, Hartlieb K, Krause H. Gas phase detection of explosives such as 2,4,6-trinitrotoluene by molecularly imprinted polymers. Anal Chim Acta. 2007 May;591(1):49-56. https://linkinghub.elsevier.com/retrieve/pii/S0003267007002905.

91. Kikuchi M, Tsuru N, Shiratori S. Recognition of terpenes using molecular imprinted polymer coated quartz crystal microbalance in air phase. Sci Technol Adv Mater. 2006 Jan 12;7(2):156-61. http://www.tandfonline.com/doi/full/10.1016/j.stam.2005.12.004.

92. Hwang M-J, Shim W-G, Yoon S-D, Moon H. Adsorption of toxic gases on molecularly imprinted polymer coated QCM: measurements and modeling for partial pressure in gas mixture. Adsorption. 2019 May 8;25(4):825-32. http://link.springer.com/ 10.1007/s10450-019-00074-w.

93. Di Natale C, Monti D, Paolesse R. Chemical sensitivity of porphyrin assemblies. Mater Today. 2010;13(7-8):46-52. https://doi. org/10.1016/S1369-7021(10)70127-9.

94. Magna G, Dinc Zor S, Catini A, Capuano R, Basoli F, Martinelli E, et al. Surface arrangement dependent selectivity of porphyrins gas sensors. Sensors Actuators, B Chem. 2017;251:524-32. https://doi.org/10.1016/j.snb.2017.05.054.

95. Paolesse R, Nardis S, Monti D, Stefanelli M, Di Natale C. Porphyrinoids for chemical sensor applications. Chem Rev. 2017 Feb 22;117(4):2517-83. https://pubs.acs.org/doi/10.1021/ acs.chemrev.6b00361.

96. Bohrer FI, Colesniuc CN, Park J, Ruidiaz ME, Schuller IK, Kummel AC, et al. Comparative gas sensing in cobalt, nickel, copper, zinc, and metal-free phthalocyanine chemiresistors. J Am Chem Soc. 2009 Jan 21;131(2):478-85. https://pubs.acs. org/doi/10.1021/ja803531r.

97. Böhmer V. Calixarenes, macrocycles with (almost) unlimited possibilities. Angew Chemie Int Ed English. 1995 Apr 13;34(7):71345. http://doi.wiley.com/10.1002/anie.199507131.

98. Ozmen M, Ozbek Z, Buyukcelebi S, Bayrakci M, Ertul S, Ersoz $\mathrm{M}$, et al. Fabrication of Langmuir-Blodgett thin films of calix[4] arenes and their gas sensing properties: Investigation of upper rim para substituent effect. Sensors Actuators B Chem. 2014 Jan;190: 502-11. http://linkinghub.elsevier.com/retrieve/pii/ S0925400513010484.

99. Kostyukevych KV, Khristosenko RV, Pavluchenko AS, Vakhula AA, Kazantseva ZI, Koshets IA, et al. A nanostructural model of ethanol adsorption in thin calixarene films. Sensors Actuators. B Chem. 2016;223:470-80. https://doi.org/10.1016/j.snb.2015.09. 123.

100. Chen C-F, Han Y. Triptycene-derived macrocyclic arenes: from calixarenes to helicarenes. Acc Chem Res. 2018 Sep 18;51(9): 
2093-106. https://pubs.acs.org/doi/10.1021/acs.accounts. $8 \mathrm{~b} 00268$.

101. Tortora L, Pomarico G, Nardis S, Martinelli E, Catini A, D’Amico A, et al. Supramolecular sensing mechanism of corrole thin films. Sensors Actuators, B Chem. 2013;187:72-7. https://doi.org/10. 1016/j.snb.2012.09.055.

102. Ozmen M, Ozbek Z, Bayrakci M, Ertul S, Ersoz M, Capan R. Preparation and gas sensing properties of Langmuir-Blodgett thin films of calix[n]arenes: Investigation of cavity effect. Sensors Actuators B Chem. 2014 May;195:156-64. http://linkinghub. elsevier.com/retrieve/pii/S0925400514000586.

103. Compagnone D, Faieta M, Pizzoni D, Di Natale C, Paolesse R, Van Caelenberg T, et al. Quartz crystal microbalance gas sensor arrays for the quality control of chocolate. Sensors Actuators, B Chem. 2015;207(PB):1114-20. https://doi.org/10.1016/j.snb. 2014.10.049.

104. Zetola N, Modongo C, Matlhagela K, Sepako E, Matsiri O, Tamuhla $\mathrm{T}$, et al. Identification of a large pool of microorganisms with an array of porphyrin based gas sensors. Sensors. $2016 \mathrm{Apr}$ 1;16(4):466. http://www.mdpi.com/1424-8220/16/4/466

105. Regmi BP, Galpothdeniya WIS, Siraj N, Webb MH, Speller NC, Warner IM. Phthalocyanine- and porphyrin-based GUMBOS for rapid and sensitive detection of organic vapors. Sensors Actuators, B Chem. 2014;209:172-9. https://doi.org/10.1016/j.snb.2014.11. 068

106. Harbeck S, Göçmen S, Emirik F, Öztürk ZZZ, Ahsen V, Gürek AGG, et al. Synthesis of branched alkoxy side chains containing phthalocyanine derivates and their application in mass sensitive QCM sensors. Sensors Actuators, B Chem. 2016 Oct;233:55-62. https://1inkinghub.elsevier.com/retrieve/pii/ S092540051630507X.

107. Harbeck M, Taşaltın C, Gürol I, Musluoğlu E, Ahsen V, Öztürk ZZ. Preferential sorption of polar compounds by fluoroalkyloxy substituted phthalocyanines for the use in sorption based gas sensors. Sensors Actuators B Chem. 2010 Oct;150(2):616-24. https:// linkinghub.elsevier.com/retrieve/pii/S0925400510006921.

108. Harbeck S, Emirik OF, Gürol I, Gurek AG, Öztürk ZZ, Ahsen V. Understanding the VOC sorption processes on fluoro alkyl substituted phthalocyanines using ATR FT-IR spectroscopy and QCM measurements. Sensors Actuators, B Chem. 2013;176:83849. https://doi.org/10.1016/j.snb.2012.08.020.

109. Hu J, Odom TW, Lieber CM. Chemistry and physics in one dimension: synthesis and properties of nanowires and nanotubes. Acc Chem Res. 1999 May;32(5):435-45. https://pubs.acs.org/ doi/10.1021/ar9700365.

110. Nanotube KJ. Molecular wires as chemical sensors. Science (80-). 2000 Jan 28;287(5453):622-5. http://www.sciencemag.org/cgi/ doi/10.1126/science.287.5453.622.

111. Hierlemann A, Ricco AJ, Bodenhöfer K, Dominik A, Göpel W. Conferring selectivity to chemical sensors via polymer side-chain selection: thermodynamics of vapor sorption by a set of polysiloxanes on thickness-shear mode resonators. Anal Chem. 2000 Aug;72(16):3696-708. http://pubs.acs.org/doi/abs/10.1021/ ac991298i.

112. Zimmermann C, Rebière D, Déjous C, Pistré J, Chastaing E, Planade R. A love-wave gas sensor coated with functionalized polysiloxane for sensing organophosphorus compounds. Sensors Actuators B Chem. 2001 Jun;76(1-3):86-94. https://linkinghub. elsevier.com/retrieve/pii/S0925400501005780.

113. Huang J, Jiang Y, Du X, Bi J. A new siloxane polymer for chemical vapor sensor. Sensors Actuators, B Chem. 2010;146(1):38894. https://doi.org/10.1016/j.snb.2010.02.010.

114. Wang L, Cha X, Wu Y, Xu J, Cheng Z, Xiang Q, et al. Superhydrophobic Polymerized n -Octadecylsilane Surface for BTEX Sensing and Stable Toluene/Water Selective Detection
Based on QCM Sensor. ACS Omega. 2018 Feb 28;3(2):243743. https://pubs.acs.org/doi/10.1021/acsomega.8b00061.

115. Kumar A, Brunet J, Varenne C, Ndiaye A, Pauly A, Penza M, et al. Tetra-tert-butyl copper phthalocyanine-based QCM sensor for toluene detection in air at room temperature. Sensors Actuators, B Chem. 2015;210:398-407. https://doi.org/10.1016/j.snb.2015.01. 010.

116. Rizzo S, Sannicolò F, Benincori T, Schiavon G, Zecchin S, Zotti G. Calix[4] arene-functionalized poly-cyclopenta[2,1-b;3,4-b ']bithiophenes with good recognition ability and selectivity for small organic molecules for application in QCM-based sensors. J Mater Chem. 2004;14(12):1804-11. http://xlink.rsc.org/?DOI= B314345G.

117. Zong J, Zhang YS, Zhu Y, Zhao Y, Zhang W, Zhu Y. Rapid and highly selective detection of formaldehyde in food using quartz crystal microbalance sensors based on biomimetic poly-dopamine functionalized hollow mesoporous silica spheres. Sensors Actuators B Chem. 2018 Oct;271:311-20. https://linkinghub. elsevier.com/retrieve/pii/S0925400518309900.

118. Zhu Y, Cheng Z, Xiang Q, Chen X, Xu J. Synthesis of functionalized mesoporous $\mathrm{TiO}_{2}-\mathrm{SiO}_{2}$ with organic fluoroalcohol as high performance DMMP gas sensor. Sensors Actuators B Chem. 2017 Sep;248:785-92. https://doi.org/10.1016/j.snb.2016.10.080.

119. Ogimoto Y, Selyanchyn R, Takahara N, Wakamatsu S, Lee S-W. Detection of ammonia in human breath using quartz crystal microbalance sensors with functionalized mesoporous $\mathrm{SiO}_{2}$ nanoparticle films. Sensors Actuators B Chem. 2015 Aug;215:428-36. http://linkinghub.elsevier.com/retrieve/pii/S0925400515004542.

120. Wang L, Wang Z, Xiang Q, Chen Y, Duan Z, Xu J. High performance formaldehyde detection based on a novel copper (II) complex functionalized QCM gas sensor. Sensors Actuators, B Chem. 2017;248:820-8. https://doi.org/10.1016/j.snb.2016.12.015.

121. Compton OC, Nguyen ST. Graphene oxide, highly reduced graphene oxide, and graphene: versatile building blocks for carbon-based materials. Small. 2010 Mar 22;6(6):711-23. http://doi. wiley.com/10.1002/smll.200901934.

122. Ebbesen TW, Lezec HJ, Hiura H, Bennett JW, Ghaemi HF, Thio T. Electrical conductivity of individual carbon nanotubes. Nature. 1996 Jul;382(6586):54-6. http://www.nature.com/articles/ $382054 \mathrm{a} 0$.

123. Baughman RH. Carbon nanotubes-the route toward applications. Science (80- ). 2002 Aug 2;297(5582):787-92. http://www. sciencemag.org/cgi/doi/10.1126/science.1060928.

124. Tasis D, Tagmatarchis N, Bianco A, Prato M. Chemistry of Carbon Nanotubes. Chem Rev. 2006 Mar;106(3):1105-36. https://pubs.acs.org/doi/10.1021/cr050569o.

125. Novoselov KS, Geim AK, Morozov SV, Jiang D, Katsnelson MI, Grigorieva IV, et al. Two-dimensional gas of massless Dirac fermions in graphene. Nature. 2005 Nov;438(7065):197-200. http:// www.nature.com/articles/nature 04233.

126. Geim AK, Novoselov KS. The rise of graphene. Nat Mater. 2007;6(3):183-91. http:/www.ncbi.nlm.nih.gov/pubmed/ 17330084

127. Du X, Skachko I, Barker A, Andrei EY. Approaching ballistic transport in suspended graphene. Nat Nanotechnol. 2008 Aug 20;3(8):491-5. http://www.nature.com/articles/nnano.2008.199.

128. Geim AK. Graphene: status and prospects. Science (80- ). 2009 Jun 19;324(5934):1530-4. http://www.sciencemag.org/cgi/doi/ 10.1126/science.1158877.

129. Novoselov KS, VI F'k, Colombo L, Gellert PR, Schwab MG, Kim K. A roadmap for graphene. Nature. 2012 Oct 10;490(7419):192200. https://doi.org/10.1038/nature11458.

130. Acik M, Chabal YJ. A review on reducing graphene oxide for band gap engineering. J Mater Sci Res. 2012 Dec;6:2(1). http:// www.ccsenet.org/journal/index.php/jmsr/article/view/20352. 
131. Rowley-Neale SJ, Randviir EP, Abo Dena AS, Banks CE. An overview of recent applications of reduced graphene oxide as a basis of electroanalytical sensing platforms. Appl Mater Today. 2018 Mar;10:218-26. https://inkinghub.elsevier.com/retrieve/pii/ S2352940717301075.

132. Hirsch A. Functionalization of single-walled carbon nanotubes. Angew Chemie Int Ed. 2002 Jun 3;41(11):1853. http://doi.wiley. $\mathrm{com} / 10.1002 / 1521-3773 \% 2820020603 \% 2941 \% 3 \mathrm{~A} 11 \%$ 3C1853\%3A\%3AAID-ANIE1853\%3E3.0.CO\%3B2-N

133. Kuila T, Bose S, Mishra AK, Khanra P, Kim NH, Lee JH. Chemical functionalization of graphene and its applications. Prog Mater Sci. 2012;57(7):1061-105. https://doi.org/10.1016/j. pmatsci.2012.03.002.

134. Li J, Lu Y, Ye Q, Cinke M, Han J, Meyyappan M. Carbon nanotube sensors for gas and organic vapor detection. Nano Lett. 2003 Jul;3(7):929-33. https://pubs.acs.org/doi/10.1021/n1034220x.

135. Yang S, Jiang C, Huai WS. Gas sensing in 2D materials. Appl Phys Rev. 2017:4(2). https://doi.org/10.1063/1.4983310.

136. Asad M, Sheikhi MH. Surface acoustic wave based $\mathrm{H}_{2} \mathrm{~S}$ gas sensors incorporating sensitive layers of single wall carbon nanotubes decorated with $\mathrm{Cu}$ nanoparticles. Sensors Actuators, B Chem. 2014;198:134-41. https://doi.org/10.1016/j.snb.2014.03.024.

137. Ndiaye AL, Brunet J, Varenne C, Pauly A. Functionalized CNTsbased gas sensors for BTX-type gases: how functional peripheral groups can affect the time response through surface reactivity. J Phys Chem C. 2018 Sep 20;122(37):21632-43. http://pubs.acs. org/doi/10.1021/acs.jpcc.8b05379.

138. Yu H, Xu P, Lee D-W, Li X. Porous-layered stack of functionalized AuNP-rGO (gold nanoparticles-reduced graphene oxide) nanosheets as a sensing material for the micro-gravimetric detection of chemical vapor. J Mater Chem A. 2013;1(14):4444. http:// xlink.rsc.org/?DOI=c3ta01401k

139. Xia X, Guo S, Zhao W, Xu P, Yu H, Xu T, et al. Carboxyl functionalized gold nanoparticles in situ grown on reduced graphene oxide for micro-gravimetric ammonia sensing. Sensors Actuators, B Chem. 2014;202:846-53. https://doi.org/10.1016/j. snb.2014.06.029.

140. Xu R, Pang W, Yu J, Huo Q, Chen J. Chemistry of zeolites and related porous materials: synthesis and structure. 1st ed. Singapore: John Wiley \& Sons (Asia), Pte Ltd; 2007. p. 678.

141. Brunsveld L, Folmer BJB, Meijer EW, Sijbesma RP. Supramolecular Polymers. Chem Rev. 2001 Dec;101(12):407198. https://pubs.acs.org/doi/10.1021/cr990125q.

142. Kresge CT, Leonowicz ME, Roth WJ, Vartuli JC, Beck JS. Ordered mesoporous molecular sieves synthesized by a liquidcrystal template mechanism. Nature. 1992 Oct;359(6397):710 2. $\mathrm{http}: / / \mathrm{www} \cdot$ nature.com/articles/359710a0.

143. Li H, Eddaoudi M, O'Keeffe M, Yaghi OM. Design and synthesis of an exceptionally stable and highly porous metal-organic framework. Nature. 1999 Nov;402(6759):276-9. http://www.nature. com/articles/46248.

144. Furukawa H, Cordova KE, O'Keeffe M, Yaghi OM. The chemistry and applications of metal-organic frameworks. Science (80-). 2013 Aug 30;341(6149):1230444-4. https://www.sciencemag. org/lookup/doi/10.1126/science.1230444.

145. Gu ZG, Zhang J. Epitaxial growth and applications of oriented metal-organic framework thin films. Coord Chem Rev. 2019;378:513-32. https://doi.org/10.1016/j.ccr.2017.09.028.

146. Somorjai GA. Directions of theoretical and experimental investigations into the mechanisms of heterogeneous catalysis. Catal Letters. 1991;9(3-4):311-28. http://link.springer.com/10.1007/ BF00773188.

147. Yaghi OM, O'Keeffe M, Ockwig NW, Chae HK, Eddaoudi M, Kim J. Reticular synthesis and the design of new materials. Nature. 2003 Jun;423(6941):705-14. http://www.nature.com/ articles/nature 01650 .
148. Wang B, Côté AP, Furukawa H, O'Keeffe M, Yaghi OM. Colossal cages in zeolitic imidazolate frameworks as selective carbon dioxide reservoirs. Nature. 2008 May;453(7192):207-11. http://www.nature.com/articles/nature06900.

149. Suh MP, Cheon YE, Lee EY. Syntheses and functions of porous metallosupramolecular networks. Coord Chem Rev. 2008 Apr;252(8-9):1007-26. https://linkinghub.elsevier.com/retrieve/ pii/S0010854508000234.

150. Xiang S, Zhou W, Gallegos JM, Liu Y, Chen B. Exceptionally High Acetylene Uptake in a Microporous Metal-Organic Framework with Open Metal Sites. J Am Chem Soc. 2009 Sep 2;131(34):12415-9. https://pubs.acs.org/doi/10.1021/ja904782h.

151. Shekhah O, Liu J, Fischer RA, Wöll C. MOF thin films: existing and future applications. Chem Soc Rev. 2011;40(2):1081. http:// xlink.rsc.org/?DOI=c0cs00147c.

152. Bloch ED, Hudson MR, Mason JA, Chavan S, Crocellà V, Howe $\mathrm{JD}$, et al. Reversible $\mathrm{CO}$ binding enables tunable $\mathrm{CO} / \mathrm{H}_{2}$ and $\mathrm{CO} /$ $\mathrm{N}_{2}$ separations in metal-organic frameworks with exposed divalent metal cations. J Am Chem Soc. 2014;136(30):10752-61. http://pubs.acs.org/doi/10.1021/ja505318p.

153. Baerlocher C, McCusker LB, Olsen DH. Atlas of zeolite framework types. 6th ed. Elsevier B.V: Amsterdam, London, New York, Oxford, Paris, Shannon, Tokyo; 2007. 398 p.

154. Zheng Y, Li X, Dutta PK. Exploitation of unique properties of zeolites in the development of gas sensors. Sensors. $2012 \mathrm{Apr}$ 20;12(4):5170-94. http://www.mdpi.com/1424-8220/12/4/5170.

155. Wales DJ, Grand J, Ting VP, Burke RD, Edler KJ, Bowen CR, et al. Gas sensing using porous materials for automotive applications. Chem Soc Rev. 2015;44(13):4290-321. http://xlink.rsc. org/?DOI=C5CS00040H.

156. Mintova S, Bein T. Nanosized zeolite films for vapor-sensing applications. Microporous Mesoporous Mater. 2001;50(2-3): 159-66. http://www.scopus.com/inward/record.url?eid=2-s2.0$0035981158 \& \mathrm{p}$ a r t n e r I D $=40 \& \mathrm{~m} \mathrm{~d} 5=$ 1c26c42436d50d1983ce9e5b12aab606.

157. Urbiztondo MA, Pellejero I, Villarroya M, Sesé J, Pina MP, Dufour I, et al. Zeolite-modified cantilevers for the sensing of nitrotoluene vapors. Sensors Actuators B Chem. 2009 Apr 2;137(2):608-16. https://linkinghub.elsevier.com/retrieve/pii/ S0925400509000549.

158. Wang L, Wu Y, Gao J, Song J. La doped AlPO-5: Enhenced $\mathrm{NH}_{3}$ sensing properties, thermodynamic investigation and humidity-enhanced effect. J Solid State Chem. 2019 Sep;277(May):54-60. https://linkinghub.elsevier.com/retrieve/pii/S0022459619302725.

159. Rosi NL, Kim J, Eddaoudi M, Chen B, O’Keeffe M, Yaghi OM. Rod packings and metal-organic frameworks constructed from rod-shaped secondary building unITS. J Am Chem Soc. 2005 Feb;127(5):1504-18. https://pubs.acs.org/doi/10.1021/ ja045123o

160. Sapsanis C, Omran H, Chernikova V, Shekhah O, Belmabkhout $\mathrm{Y}$, Buttner $\mathrm{U}$, et al. Insights on capacitive interdigitated electrodes coated with MOF thin films: humidity and VOCs sensing as a case study. Sensors. 2015 Jul 24;15(8):18153-66. http://www.mdpi. com/1424-8220/15/8/18153.

161. Yuan S, Feng L, Wang K, Pang J, Bosch M, Lollar C, et al. Stable metal-organic frameworks: design, synthesis, and applications. Adv Mater. 2018 Sep;30(37):1704303. http://doi.wiley.com/10. 1002/adma.201704303.

162. Zhao M, Huang Y, Peng Y, Huang Z, Ma Q, Zhang H. Twodimensional metal-organic framework nanosheets: Synthesis and applications. Chem Soc Rev. 2018;47(16):6267-95. https://doi. org/10.1039/c8cs00268a.

163. Han Z, Shi W, Cheng P. Synthetic strategies for chiral metalorganic frameworks. Chinese Chem Lett. 2018;29(6):819-22. https://doi.org/10.1016/j.cclet.2017.09.050. 
164. Kumar P, Deep A, Kim KH. Metal organic frameworks for sensing applications. TrAC - Trends Anal Chem. 2015;73:39-53. https://doi.org/10.1016/j.trac.2015.04.009.

165. Yi F-Y, Chen D, Wu M-K, Han L, Jiang H-L. Chemical sensors based on metal-organic frameworks. Chempluschem. 2016 Aug;81(8):675-90. http://doi.wiley.com/10.1002/cplu. 201600137

166. Achmann S, Hagen G, Kita J, Malkowsky I, Kiener C, Moos R. Metal-organic frameworks for sensing applications in the gas phase. Sensors. 2009 Mar 6;9(3):1574-89. http://www.mdpi. com/1424-8220/9/3/1574.

167. Lei J, Qian R, Ling P, Cui L, Ju H. Design and sensing applications of metal-organic framework composites. TrAC - Trends Anal Chem. 2014;58:71-8. https://doi.org/10.1016/j.trac.2014. 02.012 .

168. Campbell M, Dincă M. Metal-organic frameworks as active materials in electronic sensor devices. Sensors. 2017 May 12;17(5): 1108. http://www.mdpi.com/1424-8220/17/5/1108.

169. Li Y, Xiao A-S, Zou B, Zhang H-X, Yan K-L, Lin Y. Advances of metal-organic frameworks for gas sensing. Polyhedron. 2018 Nov;154:83-97. https://linkinghub.elsevier.com/retrieve/pii/ S0277538718304224.

170. Das MC, Xiang S, Zhang Z, Chen B. Functional mixed metalorganic frameworks with metalloligands. Angew Chemie Int Ed. 2011 Nov 4;50(45):10510-20. http://doi.wiley.com/10.1002/anie. 201101534

171. Cui Y, Li B, He H, Zhou W, Chen B, Qian G. Metal-organic frameworks as platforms for functional materials. Acc Chem Res. 2016 Mar 15;49(3):483-93. http://pubs.acs.org/doi/10. 1021/acs.accounts.5b00530.

172. Li B, Wen H-M, Cui Y, Zhou W, Qian G, Chen B. Emerging multifunctional metal-organic framework materials. Adv Mater. 2016 Oct;28(40):8819-60. http://doi.wiley.com/10.1002/adma. 201601133

173. Batten SR, Champness NR, Chen X-M, Garcia-Martinez J, Kitagawa S, Öhrström L, et al. Terminology of metal-organic frameworks and coordination polymers (IUPAC Recommendations 2013). Pure Appl Chem. 2013 Jul 31;85(8): 1715-24. http://www.degruyter.com/view/j/pac.2013.85.issue-8/ pac-rec-12-11-20/pac-rec-12-11-20.xml.

174. $\mathrm{Lv} \mathrm{Y}, \mathrm{Yu} \mathrm{H}, \mathrm{Xu} \mathrm{P}, \mathrm{Xu}$ J, Li X. Metal organic framework of MOF5 with hierarchical nanopores as micro-gravimetric sensing material for aniline detection. Sensors Actuators, B Chem. 2018;256: 639-47. https://doi.org/10.1016/j.snb.2017.09.195.

175. Yamagiwa H, Sato S, Fukawa T, Ikehara T, Maeda R, Mihara T, et al. Detection of volatile organic compounds by weight-detectable sensors coated with metal-organic frameworks. Sci Rep. 2015 May 1;4(1):6247. http://www.nature.com/articles/srep06247.

176. He Z-H, Gong S-D, Cai S-L, Yan Y-L, Chen G, Li X-L, et al. A benzimidazole-containing covalent organic framework-based QCM sensor for exceptional detection of CEES. Cryst Growth Des. 2019 Jun 5;19(6):3543-50. http://pubs.acs.org/doi/10.1021/ acs.cgd.9b00409.

177. Paschke B, Wixforth A, Denysenko D, Volkmer D. Fast surface acoustic wave-based sensors to investigate the kinetics of gas uptake in ultra-microporous frameworks. ACS Sensors. 2017 Jun 23;2(6):740-7. https://pubs.acs.org/doi/10.1021/acssensors. $7 \mathrm{~b} 00014$.

178. Regmi BP, Monk J, El-Zahab B, Das S, Hung FR, Hayes DJ, et al. A novel composite film for detection and molecular weight determination of organic vapors. J Mater Chem. 2012;22(27):13732. $\mathrm{http}: / / x$ link.rsc.org/?DOI=c2jm31623d.

179. Lal G, Tiwari DC. Investigation of nanoclay doped polymeric composites on piezoelectric quartz crystal microbalance (QCM) sensor. Sensors Actuators, B Chem. 2018;262:64-9. https://doi. org/10.1016/j.snb.2018.01.200.
180. Chen W, Wang Z, Gu S, Wang J. Detection of hexanal in humid circumstances using hydrophobic molecularly imprinted polymers composite. Sensors Actuators B Chem. 2019 Jul;291(April):1417. https://linkinghub.elsevier.com/retrieve/pii/ S092540051930588X

181. Jia Y, Chen L, Yu H, Zhang Y, Dong F. Graphene oxide/polystyrene composite nanofibers on quartz crystal microbalance electrode for the ammonia detection. RSC Adv. 2015;5(51):40620 7. https://doi.org/10.1039/C5RA04890G.

182. Zhang K, Hu R, Fan G, Li G. Graphene oxide/chitosan nanocomposite coated quartz crystal microbalance sensor for detection of amine vapors. Sensors Actuators, B Chem. 2017;243:721-30. https://doi.org/10.1016/j.snb.2016.12.063.

183. Tai H, Zhen Y, Liu C, Ye Z, Xie G, Du X, et al. Facile development of high performance QCM humidity sensor based on protonated polyethylenimine-graphene oxide nanocomposite thin film. Sensors Actuators B Chem. 2016 Jul;230:501-9. https:// doi.org/10.1016/j.snb.2016.01.105.

184. Tang S, Ma W, Xie G, Su Y, Jiang Y. Acetylcholinesterase-reduced graphene oxide hybrid films for organophosphorus neurotoxin sensing via quartz crystal microbalance. Chem Phys Lett. 2016 Sep;660:199-204. http://linkinghub.elsevier.com/retrieve/ pii/S0009261416305905.

185. Yuan Z, Tai H, Bao X, Liu C, Ye Z, Jiang Y. Enhanced humiditysensing properties of novel graphene oxide/zinc oxide nanoparticles layered thin film QCM sensor. Mater Lett. 2016 Jul;174:2831.http://linkinghub.elsevier.com/retrieve/pii/ S0167577X16301227.

186. Yuan Z, Tai H, Ye Z, Liu C, Xie G, Du X, et al. Novel highly sensitive QCM humidity sensor with low hysteresis based on graphene oxide (GO)/poly(ethyleneimine) layered film. Sensors Actuators, B Chem. 2016;234:145-54. https://doi.org/10.1016/j. snb.2016.04.070.

187. Wang L, Yu Y, Xiang Q, Xu J, Cheng Z, Xu J. PODS-covered PDA film based formaldehyde sensor for avoiding humidity false response. Sensors Actuators, B Chem. 2018;255:2704-12. https:// doi.org/10.1016/j.snb.2017.09.082.

188. Jin X, Yu L, Garcia D, Ren RX, Zeng X. Ionic liquid high-temperature gas sensor array. Anal Chem. 2006 Oct;78(19):6980-9. https://pubs.acs.org/doi/10.1021/ac0608669.

189. Li H-Y, Chu Y-H. exploiting solvate ionic liquids for amine gas analysis on a quartz crystal microbalance. Anal Chem. 2017 May 16;89(10):5186-92. http://pubs.acs.org/doi/10.1021/acs. analchem. $7 \mathrm{~b} 00857$.

190. Xu X, Li C, Pei K, Zhao K, Zhao Z, Li H. Ionic liquids used as QCM coating materials for the detection of alcohols. Sensors Actuators B Chem. 2008 Aug 28;134(1):258-65. http:// linkinghub.elsevier.com/retrieve/pii/S0925400508003225.

191. Cotton AF. Chemical applications of group theory. $3^{\text {rd }}$ Ed. WileyInterscience; 1990; $480 \mathrm{p}$.

192. Schurig V. Separation of enantiomers by gas chromatography. J Chromatogr A. 2001 Jan;906(1-2):275-99. https://linkinghub. elsevier.com/retrieve/pii/S0021967300005057.

193. Schurig V. Differentiation of Enantiomers II. Cham: Springer International Publishing; 2013. (Topics in Current Chemistry; vol. 341). http://link.springer.com/10.1007/978-3-319-03716-5.

194. Cram DJ, Helgeson RC, Sousa LR, Timko JM, Newcomb M, Moreau $\mathrm{P}$, et al. Chiral recognition in complexation of guests by designed host molecules. Pure Appl Chem. 1975 Jan 1;43(3-4): 327-349. http://www.degruyter.com/view/j/pac.1975.43.issue-34/pac197543030327/pac197543030327.xml.

195. Zhang XX, Bradshaw JS, Izatt RM. Enantiomeric recognition of amine compounds by chiral macrocyclic receptors. Chem Rev. 1997 Dec;97(8):3313-62. http://pubs.acs.org/doi/abs/10.1021/ cr960144p. 
196. Maier NM, Lindner W. Chiral recognition applications of molecularly imprinted polymers: a critical review. Anal Bioanal Chem. 2007 Aug 23;389(2):377-97. http://link.springer.com/10.1007/ s00216-007-1427-4.

197. Zehnacker A, Suhm MA. Chirality recognition between neutral molecules in the gas phase. Angew Chemie Int Ed. 2008 Sep 1;47(37):6970-92. http://doi.wiley.com/10.1002/anie. 200800957.

198. Scriba GKE. Chiral recognition mechanisms in analytical separation sciences. Chromatographia. 2012 Aug 2;75(15-16):815-38. http:/link.springer.com/10.1007/s10337-012-2261-1.

199. Scriba GKE. Chiral recognition in separation science - an update. J Chromatogr A. 2016 Oct;1467:56-78. https://doi.org/10.1016/j. chroma.2016.05.061.

200. Freire F, Quiñoá E, Riguera R. Supramolecular assemblies from poly(phenylacetylene)s. Chem Rev. 2016 Feb 10;116(3):124271. https://pubs.acs.org/doi/10.1021/acs.chemrev.5b00280.

201. Chen L-J, Yang H-B, Shionoya M. Chiral metallosupramolecular architectures. Chem Soc Rev. 2017;46(9):2555-76. http://xlink. rsc.org/?DOI=C7CS00173H.

202. Guo W, Wang J, Wang C, He J-Q, He X, Cheng J-P. Design, synthesis, and enantiomeric recognition of dicyclodipeptide-bearing calix[4]arenes: a promising family for chiral gas sensor coatings. Tetrahedron Lett. 2002 Aug;43(32):5665-7. https:// linkinghub.elsevier.com/retrieve/pii/S0040403902010791.

203. Ma W, Cheng F, Liu Y. Deep-learning-enabled on-demand design of chiral metamaterials. ACS Nano. 2018 Jun 26;12(6):6326-34. https://pubs.acs.org/doi/10.1021/acsnano.8b03569.

204. Pan M, Wu K, Zhang JH, Su CY. Chiral metal-organic cages/ containers (MOCs): From structural and stereochemical design to applications. Coord Chem Rev. 2019;378:333-49. https://doi. org/10.1016/j.ccr.2017.10.031.

205. Bodenhöfer K, Hierlemann A, Seemann J, Gauglitz G, Christian B, Koppenhoefer B, et al. Chiral discrimination in the gas phase using different transducers: thickness shear mode resonators and reflectometric interference spectroscopy. Anal Chem. 1997 Aug;69(15):3058-68. https://pubs.acs.org/doi/10.1021/ ac9612990.

206. Bodenhöfer K, Hierlemann A, Seemann J, Gauglitz G, Koppenhoefer B, Gpel W. Chiral discrimination using piezoelectric and optical gas sensors. Nature. 1997 Jun 5;387(6633):57780. http://www.nature.com/articles/42426.

207. Bodenhöfer K, Hierlemann A, Juza M, Schurig V, Göpel W. Chiral discrimination of inhalation anesthetics and methyl propionates by thickness shear mode resonators: new insights into the mechanisms of enantioselectivity by cyclodextrins. Anal Chem. 1997 Oct;69(19):4017-31. http://pubs.acs.org/doi/abs/10.1021/ ac970367v

208. Easton CJ, Lincoln SF. Chiral discrimination by modified cyclodextrins. Chem Soc Rev. 1996;25(3):163. http://xlink.rsc.org/? DOI $=$ cs9962500163.

209. Rekharsky M, Inoue Y. Chiral recognition thermodynamics of $\beta$ Cyclodextrin: the thermodynamic origin of enantioselectivity and the enthalpy-entropy compensation effect. J Am Chem Soc. 2000 May;122(18):4418-35. https://pubs.acs.org/doi/10.1021/ ja9921118.

210. Fietzek C, Hermle T, Rosenstiel W, Schurig V. Chiral discrimination of limonene by use of $\beta$-cyclodextrin-coated quartz-crystalmicrobalances (QCMs) and data evaluation by artificial neuronal networks. Fresenius J Anal Chem. 2001 Sep 18;371(1):58-63. $\mathrm{http} / / /$ link.springer.com/10.1007/s002160100899.

211. Kieser B, Fietzek C, Schmidt R, Belge G, Weimar U, Schurig V, et al. Use of a modified cyclodextrin host for the enantioselective detection of a halogenated diether as chiral guest via optical and electrical transducers. Anal Chem. 2002 Jul;74(13):3005-12. http://pubs.acs.org/doi/abs/10.1021/ac015689k.
212. Xu C, Ng SC, Chan HSO. Self-assembly of perfunctionalized $\beta$ cyclodextrins on a quartz crystal microbalance for real-time chiral recognition. Langmuir. 2008 Aug;24(16):9118-24. https://pubs. acs.org/doi/10.1021/la800761h.

213. Paolesse R, Monti D, La Monica L, Venanzi M, Froiio A, Nardis $\mathrm{S}$, et al. Preparation and self-assembly of chiral porphyrin diads on the gold electrodes of quartz crystal microbalances: a novel potential approach to the development of enantioselective chemical sensors. Chem - A Eur J. 2002 Jun 3;8(11):2476. http://doi.wiley. com/10.1002/1521-3765\%2820020603\%298\%3A11\%3C2476\% 3A\%3AAID-CHEM2476\%3E3.0.CO\%3B2-E.

214. Stefanelli M, Magna G, Zurlo F, Caso FM, Di Bartolomeo E, Antonaroli S, et al. Chiral selectivity of porphyrin-ZnO nanoparticle conjugates. ACS Appl Mater Interfaces. 2019 Mar 27;11(12): 12077-87. http://pubs.acs.org/doi/10.1021/acsami.8b22749.

215. Shen J, Okamoto Y. Efficient separation of enantiomers using stereoregular chiral polymers. Chem Rev. 2016 Feb 10;116(3): 1094-138. http://pubs.acs.org/doi/10.1021/acs.chemrev.5b00317.

216. Lange D, Hagleitner C, Hierlemann A, Brand O, Baltes H. Complementary metal oxide semiconductor cantilever arrays on a single chip: mass-sensitive detection of volatile organic compounds. Anal Chem. 2002 Jul;74(13):3084-95. http://pubs.acs. org/doi/abs/10.1021/ac011269j.

217. Jin X, Huang Y, Mason A, Zeng X. Multichannel monolithic quartz crystal microbalance gas sensor array. Anal Chem. 2009 Jan 15;81(2):595-603. http://pubs.acs.org/doi/abs/10.1021/ ac8018697.

218. Tao W, Lin P, Ai Y, Wang H, Ke S, Zeng X. Multichannel quartz crystal microbalance array: Fabrication, evaluation, application in biomarker detection. Anal Biochem. 2016;494:85-92. https://doi. org/10.1016/j.ab.2015.11.001.

219. Tuantranont A, Wisitsora-at A, Sritongkham P, Jaruwongrungsee $\mathrm{K}$. A review of monolithic multichannel quartz crystal microbalance: A review. Anal Chim Acta. 2011;687(2):114-28. http:// www.scopus.com/inward/record.url? eid=2-s 2.0 $79151476256 \&$ p a r t n e r I D $=40 \&$ m d $5=$ d914cf48eee8dfae98c8f13ac534cc34.

220. Röck F, Barsan N, Weimar U. Electronic nose: current status and future trends. Chem Rev. 2008 Feb;108(2):705-25. https://pubs. acs.org/doi/10.1021/cr068121q.

221. Beccherelli R, Zampetti E, Pantalei S, Bernabei M, Persaud KC Design of a very large chemical sensor system for mimicking biological olfaction. Sensors Actuators, B Chem. 2010;146(2): 446-52. https://doi.org/10.1016/j.snb.2009.11.031.

222. Wilson AD. Review of electronic-nose technologies and algorithms to detect hazardous chemicals in the environment. Procedia Technol. 2012;1:453-63. https://doi.org/10.1016/j. protcy.2012.02.101.

223. Marco S, Gutiérrez-Gálvez A, Lansner A, Martinez D, Rospars JP, Beccherelli R, et al. A biomimetic approach to machine olfaction, featuring a very large-scale chemical sensor array and embedded neuro-bio-inspired computation. Microsyst Technol. 2014 Apr 21;20(4-5):729-42. http://link.springer.com/10.1007/s00542013-2020-8.

224. Wasilewski T, Gębicki J, Kamysz W. Bioelectronic nose: Current status and perspectives. Biosens Bioelectron. 2017 Jan;87:480 94. https://linkinghub.elsevier.com/retrieve/pii/ S0956566316308375.

225. Pelosi P, Zhu J, Knoll W. From gas sensors to biomimetic artificial noses. Chemosensors. 2018 Aug 7;6(3):32. http://www.mdpi. com/2227-9040/6/3/32.

226. Hierlemann A, Weimar U, Kraus G, Schweizer-Berberich M, Göpel W. Polymer-based sensor arrays and multicomponent analysis for the detection of hazardous oragnic vapours in the environment. Sensors Actuators B Chem. 1995 Jan;26(1-3):126-34. https://linkinghub.elsevier.com/retrieve/pii/0925400594015724. 
227. Kim BH, Prins FE, Kern DP, Raible S, Weimar U. Multicomponent analysis and prediction with a cantilever array based gas sensor. Sensors Actuators B Chem. 2001 Aug;78(1-3): 12-8. https://linkinghub.elsevier.com/retrieve/pii/ S0925400501007857.

228. Dickert FL, Hayden O, Zenkel ME. Detection of volatile compounds with mass-sensitive sensor arrays in the presence of variable ambient humidity. Anal Chem. 1999 Apr;71(7):1338-41. https://pubs.acs.org/doi/10.1021/ac981014e.

229. Matatagui D, Fernández MJ, Fontecha J, Santos JP, Gràcia I, Cané $\mathrm{C}$, et al. Love-wave sensor array to detect, discriminate and classify chemical warfare agent simulants. Sensors Actuators, B Chem. 2012;175:173-8. https://doi.org/10.1016/j.snb.2012.02. 061.

230. Senesac LR, Dutta P, Datskos PG, Sepaniak MJ. Analyte species and concentration identification using differentially functionalized microcantilever arrays and artificial neural networks. Anal Chim Acta. 2006 Feb;558(1-2):94-101. https://linkinghub.elsevier. com/retrieve/pii/S0003267005018842.

231. Bachar N, Liberman L, Muallem F, Feng X, Müllen K, Haick H. Sensor arrays based on polycyclic aromatic hydrocarbons: chemiresistors versus quartz-crystal microbalance. ACS Appl Mater Interfaces. 2013 Nov 27;5(22):11641-53. https://pubs.acs. org/doi/10.1021/am403067t.

232. Lu Y, Chang Y, Tang N, Qu H, Liu J, Pang W, et al. Detection of volatile organic compounds using microfabricated resonator array functionalized with supramolecular monolayers. ACS Appl Mater Interfaces. 2015 Aug 19;7(32):17893-903. https://pubs.acs.org/ doi/10.1021/acsami.5b04385.

233. Dubbeldam D, Calero S, Ellis DE, Snurr RQ. RASPA: molecular simulation software for adsorption and diffusion in flexible nanoporous materials. Mol Simul. 2016 Jan 22;42(2):81-101. http://www.tandfonline.com/doi/full/10.1080/08927022.2015. 1010082.

234. Gustafson JA, Wilmer CE. Optimizing information content in MOF sensor arrays for analyzing methane-air mixtures. Sensors Actuators, B Chem. 2018;267:483-93. https://doi.org/10.1016/j. snb.2018.04.049.

235. Kullback S, Leibler RA. On information and sufficiency. Ann Math Stat. 1951 Mar;22(1):79-86. http://projecteuclid.org/ euclid.aoms/1177729694.

236. Speller NC, Siraj N, Regmi BP, Marzoughi H, Neal C, Warner IM. Rational design of QCM-D virtual sensor arrays based on film thickness, viscoelasticity, and harmonics for vapor discrimination. Anal Chem. 2015 May 19;87(10):5156-66. http://pubs.acs.org/ doi/10.1021/ac5046824

237. Speller NC, Siraj N, McCarter KS, Vaughan S, Warner IM. QCM virtual sensor array: Vapor identification and molecular weight approximation. Sensors Actuators, B Chem. 2017;246:952-60. https://doi.org/10.1016/j.snb.2017.02.042.

238. Zeng G, Wu C, Chang Y, Zhou C, Chen B, Zhang M, et al. Detection and discrimination of volatile organic compounds using a single film bulk acoustic wave resonator with temperature modulation as a multiparameter virtual sensor array. ACS Sensors. 2019 Jun 28;4(6):1524-33. https://pubs.acs.org/doi/10.1021/ acssensors.8b01678.

239. Chen X, Cao M, Li Y, Hu W, Wang P, Ying K, et al. A study of an electronic nose for detection of lung cancer based on a virtual SAW gas sensors array and imaging recognition method. Meas Sci Technol. 2005 Aug 1;16(8):1535-46. http://stacks.iop.org/ $0957-0233 / 16 / \mathrm{i}=8 / \mathrm{a}=001$ ? k e y=cross ref. 6f21e9eeb6e7b175581b895060c8eae7.

240. Fraiwan A, Lee H, Choi S. A paper-based cantilever array sensor: Monitoring volatile organic compounds with naked eye. Talanta. 2016;158:57-62. https://doi.org/10.1016/j.talanta.2016.05.048.
241. Xu X, Cang H, Li C, Zhao ZK, Li H. Quartz crystal microbalance sensor array for the detection of volatile organic compounds. Talanta. 2009 May 15;78(3):711-6. https://linkinghub.elsevier. com/retrieve/pii/S003991400800917X.

242. Lu H-H, Rao YK, Wu T-Z, Tzeng Y-M. Direct characterization and quantification of volatile organic compounds by piezoelectric module chips sensor. Sensors Actuators B Chem. 2009 Apr 2;137(2):741-6. https://linkinghub.elsevier.com/retrieve/pii/ S0925400509001002.

243. Harbeck M, Șen Z, Gümüș G, Gürol I, Musluoğlu E, Öztürk ZZ, et al. Customized vic-dioximes and their metal complexes for enhanced chemical sensing of polar organic molecules. Sensors Actuators B Chem. 2013 Nov;188:1004-11. https://inkinghub. elsevier.com/retrieve/pii/S0925400513009374.

244. Hou K-Y, Rehman A, Zeng X. Study of ionic liquid immobilization on polyvinyl ferrocene substrates for gas sensor arrays. Langmuir. 2011 Apr 19;27(8):5136-46. https://pubs.acs.org/doi/ 10.1021/la104191n.

245. Rehman A, Hamilton A, Chung A, Baker GA, Wang Z, Zeng X. Differential solute gas response in ionic-liquid-based qcm arrays: elucidating design factors responsible for discriminative explosive gas sensing. Anal Chem. 2011 Oct 15;83(20):7823-33. https:// pubs.acs.org/doi/10.1021/ac201583c.

246. Selyanchyn R, Wakamatsu S, Hayashi K, Lee S-W. A nano-thin film-based prototype qcm sensor array for monitoring human

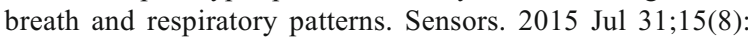
18834-50. http://www.mdpi.com/1424-8220/15/8/18834.

247. Jha SK, Hayashi K. A quick responding quartz crystal microbalance sensor array based on molecular imprinted polyacrylic acids coating for selective identification of aldehydes in body odor. Talanta. 2015;134:105-19. https://doi.org/10.1016/j.talanta.2014. 09.049 .

248. Jha SK, Liu C, Hayashi K. Molecular imprinted polyacrylic acids based QCM sensor array for recognition of organic acids in body odor. Sensors Actuators, B Chem. 2014;204:74-87. https://doi. org/10.1016/j.snb.2014.07.098.

249. Mascini M, Gaggiotti S, Della Pelle F, Di Natale C, Qakala S, Iwuoha $\mathrm{E}$, et al. Peptide modified $\mathrm{ZnO}$ nanoparticles as gas sensors array for volatile organic compounds (VOCs). Front Chem. $2018 \mathrm{Apr} ; 16: 6$. http://journal.frontiersin.org/article/10.3389/ fchem.2018.00105/full.

250. Iqbal N, Mustafa G, Rehman A, Biedermann A, Najafi B, Lieberzeit PA, et al. QCM-arrays for sensing terpenes in fresh and dried herbs via bio-mimetic mip layers. Sensors. 2010 Jun 28;10(7):6361-76. http://www.mdpi.com/1424-8220/10/7/6361.

251. Su P-G, Chuang T-Y. Simple and rapid differentiation of toxic gases using a quartz crystal microbalance sensor array coupled with principal component analysis. Sensors Actuators A Phys. 2017 Aug;263:1-7. https://linkinghub.elsevier.com/retrieve/pii/ S0924424717302157.

252. Muckley ES, Anazagasty C, Jacobs CB, Hianik T, Ivanov IN. Low-cost scalable quartz crystal microbalance array for environmental sensing. In: Kymissis I, Shinar R, Torsi L, editors. 2016. p. 99440Y. http://proceedings.spiedigitallibrary.org/proceeding. aspx?doi=10.1117/12.2237942.

253. Then D, Vidic A, Ziegler C. A highly sensitive self-oscillating cantilever array for the quantitative and qualitative analysis of organic vapor mixtures. Sensors Actuators B Chem. 2006 Sep;117(1):1-9. https://linkinghub.elsevier.com/retrieve/pii/ S0925400505007616.

254. Possas-Abreu M, Ghassemi F, Rousseau L, Scorsone E, Descours E, Lissorgues G. Development of diamond and silicon mems sensor arrays with integrated readout for vapor detection. Sensors. 2017 May 24;17(6):1163. http://www.mdpi.com/1424-8220/17/ 6/1163. 
255. Wen W, Shitang H, Shunzhou L, Minghua L, Yong P. Enhanced sensitivity of SAW gas sensor coated molecularly imprinted polymer incorporating high frequency stability oscillator. Sensors Actuators B Chem. 2007 Aug;125(2):422-7. https://inkinghub. elsevier.com/retrieve/pii/S0925400507001426.

256. Hierlemann A, Lange D, Hagleitner C, Kerness N, Koll A, Brand $\mathrm{O}$, et al. Application-specific sensor systems based on CMOS chemical microsensors. Sensors Actuators B Chem. 2000 Nov;70(1-3):2-11. http://linkinghub.elsevier.com/retrieve/pii/ S0925400500005463.

257. Hagleitner C, Hierlemann A, Lange D, Kummer A, Kerness N, Brand O, et al. Smart single-chip gas sensor microsystem. Nature. 2001 Nov;414(6861):293-6. http://www.nature.com/articles/ 35104535.

258. Hierlemann A, Baltes H. CMOS-based chemical microsensors. Analyst. 2003 Dec 23;128(1):15-28. http://xlink.rsc.org/?DOI= b208563c.

259. Lu HL, Lu CJ, Tian WC, Sheen HJ. A vapor response mechanism study of surface-modified single-walled carbon nanotubes coated chemiresistors and quartz crystal microbalance sensor arrays. Talanta. 2015;131:467-74. https://doi.org/10.1016/j.talanta.2014. 08.027 .

260. Penza M, Cassano G, Aversa P, Antolini F, Cusano A, Consales $\mathrm{M}$, et al. Carbon nanotubes-coated multi-transducing sensors for VOCs detection. Sensors Actuators B Chem. 2005 Nov;(SUPPL): 111, 171-2, 180. https://linkinghub.elsevier.com/retrieve/pii/ S0925400505006076.

261. Ulmer H, Mitrovics J, Noetzel G, Weimar U, Göpel W. Odours and flavours identified with hybrid modular sensor systems ${ }^{1}$. Sensors Actuators B Chem. 1997 Sep;43(1-3):24-33. https:// linkinghub.elsevier.com/retrieve/pii/S0925400597001615.

262. McGill RA, Nguyen VK, Chung R, Shaffer RE, DiLella D, Stepnowski JL, et al. The "NRL-SAWRHINO": a nose for toxic gases. Sensors Actuators B Chem. 2000 Jun;65(1-3):10-3. https://linkinghub.elsevier.com/retrieve/pii/S0925400599003524.

263. Lang HP, Ramseyer JP, Grange W, Braun T, Schmid D, Hunziker $\mathrm{P}$, et al. An artificial nose based on microcantilever array sensors. J Phys Conf Ser. 2007 Mar 1;61(1):663-7. http://stacks.iop.org/ $1742-6596 / 61 / \mathrm{i}=1 / \mathrm{a}=133$ ? ke y = cross ref. 95147efc311ae631a289dec3b24f171d.

264. Leis J, Zhao W, Pinnaduwage LA, Gehl AC, Allman SL, Shepp A, et al. Estimating gas concentration using a microcantileverbased electronic nose. Digit Signal Process A Rev J. 2010;20(4): 1229-37. https://doi.org/10.1016/j.dsp.2009.10.026.

265. Fernandez M, Fontecha J, Sayago I, Aleixandre M, Lozano J, Gutierrez J, et al. Discrimination of volatile compounds through an electronic nose based on ZnO SAW sensors. Sensors Actuators B Chem. 2007 Oct 20;127(1):277-83. https://linkinghub.elsevier. com/retrieve/pii/S0925400507004984.

266. Rivai M, Purwanto D, Juwono H, Sujono HA. Electronic nose using gas chromatography column and quartz crystal microbalance. TELKOMNIKA (Telecommunication Comput Electron Control). 2011 Aug 1;9(2):319. http://journal.uad.ac.id/index. php/TELKOMNIKA/article/view/703.

267. Jung Lu C, Zellers ET. Multi-adsorbent preconcentration/focusing module for portable-GC/microsensor-array analysis of complex vapor mixtures. Analyst. 2002 Aug 1;127(8):1061-8. http:// xlink.rsc.org/?DOI=b111689d.

268. Bender F, Barié N, Romoudis G, Voigt A, Rapp M. Development of a preconcentration unit for a SAW sensor micro array and its use for indoor air quality monitoring. Sensors Actuators B Chem. 2003 Aug;93(1-3):135-41. https://linkinghub.elsevier.com/ retrieve/pii/S0925400503002399.

269. Beeley JM, Mills C, Hammond PA, Glidle A, Cooper JM, Wang $\mathrm{L}$, et al. All-digital interface ASIC for a QCM-based electronic nose. Sensors Actuators B Chem. 2004 Sep;103(1-2):31-6. http://linkinghub.elsevier.com/retrieve/pii/S0925400504001212.

270. Fanget S, Hentz S, Puget P, Arcamone J, Matheron M, Colinet E, et al. Gas sensors based on gravimetric detection-A review. Sensors Actuators B Chem. 2011 Dec;160(1):804-21. http:// linkinghub.elsevier.com/retrieve/pii/S0925400511007891.

271. Panneerselvam G, Thirumal V, Pandya HM. Review of surface acoustic wave sensors for the detection and identification of toxic environmental gases/vapours. Arch Acoust. 2018;43(3):357-67. http://journals.pan.pl/dlibra/docmetadata?id=108108DOI-10. 24425/123908.

272. Oluwatosin Abegunde O, Titilayo Akinlabi E, Philip Oladijo O, Akinlabi S, Uchenna UA. Overview of thin film deposition techniques. AIMS Mater Sci. 2019;6(2):174-99. http://www. aimspress.com/article/10.3934/matersci.2019.2.174.

273. Seshan K, Schepis D, editors. Handbook of thin film deposition. fourth. Elsevier; 2018. 470 p. https://linkinghub.elsevier.com/ retrieve/pii/C20160032436.

274. Jilani A, Abdel-wahab MS, Hammad AH. Advance deposition techniques for thin film and coating, Modern technologies for creating the thin-film systems and coatings: InTech; 2017. http:/ www.intechopen.com/books/modern-technologies-for-creatingthe-thin-film-systems-and-coatings/advance-depositiontechniques-for-thin-film-and-coating.

275. Martin PM. Handbook of deposition technologies for films and coatings. 3rd ed: Elsevier Inc; 2010. p. 936.

276. Ohring M. Materials science of thin films: Elsevier Science; 2002. p. 794. https://books.google.de/books?id=EX_LDQAAQBAJ.

277. George J. Preparation of thin films. 1st ed: CRC Press; 1992. p. 392.

278. Smith D. Thin-film deposition. 1st ed: McGraw-Hill Education; 1995. p. 616.

279. Petty MC, Barlow WA. Film Deposition. In: Roberts G, editor. Langmuir-Blodgett Films. Boston, MA: Springer US; 1990. p. 93-132. http://link.springer.com/10.1007/978-1-4899-3716-2_3.

280. Diao Y, Shaw L, Bao Z, Mannsfeld SCB. Morphology control strategies for solution-processed organic semiconductor thin films. Energy Environ Sci. 2014;7(7):2145-59. http://xlink.rsc.org/? DOI=C4EE00688G.

281. Das R, Pradhan S, Biswas S, Sharma P, Ghosh A, Bandyopadhyay $\mathrm{R}$, et al. Aliphatic amines vapours detection by quartz crystal microbalance sensor. Sensors Actuators, B Chem. 2014;198:94-101. https://doi.org/10.1016/j.snb.2014.03.020.

282. Ayad MM, Salahuddin NA, Minisy IM, Amer WA. Chitosan/ polyaniline nanofibers coating on the quartz crystal microbalance electrode for gas sensing. Sensors Actuators, B Chem. 2014;202: 144-53. https://doi.org/10.1016/j.snb.2014.05.046.

283. Ihdene Z, Mekki A, Mettai B, Mahmoud R, Hamada B, Chehimi MM. Quartz crystal microbalance VOCs sensor based on dip coated polyaniline emeraldine salt thin films. Sensors Actuators, B Chem. 2014;203:647-54. https://doi.org/10.1016/j.snb.2014.07. 030 .

284. Krebs FC. Fabrication and processing of polymer solar cells: A review of printing and coating techniques. Sol Energy Mater Sol Cells. 2009 Apr;93(4):394-412. http://linkinghub.elsevier.com/ retrieve/pii/S0927024808003486.

285. Abdellah A, Fabel B, Lugli P, Scarpa G. Spray deposition of organic semiconducting thin-films: Towards the fabrication of arbitrary shaped organic electronic devices. Org Electron. 2010 Jun;11(6):1031-8. https://linkinghub.elsevier.com/retrieve/pii/ S1566119910000844.

286. Moridi A, Hassani-Gangaraj SM, Guagliano M, Dao M. Cold spray coating: review of material systems and future perspectives. Surf Eng. 2014 Jun 16;30(6):369-95. http://www.tandfonline. com/doi/full/10.1179/1743294414Y.0000000270. 
287. Zhang D, Wang D, Zong X, Dong G, Zhang Y. High-performance QCM humidity sensor based on graphene oxide/tin oxide/ polyaniline ternary nanocomposite prepared by in-situ oxidative polymerization method. Sensors Actuators B Chem. 2018 Jun;262:531-41. http://linkinghub.elsevier.com/retrieve/pii/ S092540051830282X.

288. Gates BD, Xu Q, Stewart M, Ryan D, Willson CG, Whitesides GM. New approaches to nanofabrication: molding, printing, and other techniques. Chem Rev. 2005 Apr;105(4):1171-96. https:// pubs.acs.org/doi/10.1021/cr030076o.

289. Liu M, Guo S, Xu P, Yu H, Xu T, Zhang S, et al. Revealing humidity-enhanced $\mathrm{NH}_{3}$ sensing effect by using resonant microcantilever. Sensors Actuators, B Chem. 2018;257:488-95. https://doi.org/10.1016/j.snb.2017.10.179.

290. Salata O. Tools of nanotechnology: electrospray. Curr Nanosci. 2005 Jan 1;1(1):25-33. http://www.eurekaselect.com/openurl/ content.php?genre $=$ article $\&$ issn $=1573-4137 \&$ volume $=1 \&$ issue $=$ $1 \&$ spage $=25$.

291. Jaworek A. Electrospray droplet sources for thin film deposition. J Mater Sci. 2007 Jan 17;42(1):266-97. http://link.springer.com/10. 1007/s10853-006-0842-9.

292. Li D, Xia Y. Electrospinning of nanofibers: reinventing the wheel? Adv Mater. 2004 Jul 19;16(14):1151-70. http://doi.wiley.com/10. 1002/adma.200400719.

293. Schiffman JD, Schauer CL. A review: electrospinning of biopolymer nanofibers and their applications. Polym Rev. 2008 May;48(2):317-52. http://www.tandfonline.com/doi/abs/10. 1080/15583720802022182.

294. Jia Y, Yan C, Yu H, Chen L, Dong F. One-step fabrication of ammonia sensor by electrospinning PS-b-PMA nanofibers on quartz crystal microbalance. Sensors Actuators, B Chem. 2014;203:459-64. https://doi.org/10.1016/j.snb.2014.06.018.

295. Jia Y, Yu H, Cai J, Li Z, Dong F. Explore on the quantitative analysis of specific surface area on sensitivity of polyacrylic acid-based QCM ammonia sensor. Sensors Actuators, B Chem. 2017;243:1042-5. https://doi.org/10.1016/j.snb.2016.12.090.

296. Wang X, Ding B, Yu J, Si Y, Yang S, Sun G. Electro-netting: Fabrication of two-dimensional nano-nets for highly sensitive trimethylamine sensing. Nanoscale. 2011;3(3):911-5. http:// xlink.rsc.org/?DOI=C0NR00783H.

297. Ulman A. An introduction to ultrathin organic films: from Langmuir-Blodgett to self-assambly. San Diego, London: Academic Press Inc; 1991. 442 p.

298. Cheng JH, Fou AF, Rubner MF. Molecular self-assembly of conducting polymers. Thin Solid Films. 1994 May;244(1-2): 985-9. https://linkinghub.elsevier.com/retrieve/pii/ 0040609094906165.

299. Ariga K, Hill JP, Lee MV, Vinu A, Charvet R, Acharya S. Challenges and breakthroughs in recent research on self-assembly. Sci Technol Adv Mater. 2008 Jan 12;9(1):014109. http:// www.tandfonline.com/doi/full/10.1088/1468-6996/9/1/014109.

300. Albert JNL, Epps TH. Self-assembly of block copolymer thin films. Mater Today. 2010 Jun;13(6):24-33. https://linkinghub. elsevier.com/retrieve/pii/S1369702110701061.

301. Ariga K, Yamauchi Y, Mori T, Hill JP. 25th Anniversary article: what can be done with the langmuir-blodgett method? Recent developments and its critical role in materials science. Adv Mater. 2013 Dec;25(45):6477-512. http://doi.wiley.com/10. 1002/adma.201302283.

302. Xie J, Wang H, Duan M. QCM chemical sensor based on $\mathrm{ZnO}$ colloid spheres for the alcohols. Sensors Actuators, B Chem. 2014;203:239-44. https://doi.org/10.1016/j.snb.2014.06.119.

303. Guo S, Xu P, Yu H, Li X, Cheng Z. Hyper-branch sensing polymer batch self-assembled on resonant micro-cantilevers with a coupling-reaction route. Sensors Actuators, B Chem. 2015;209: 943-50. https://doi.org/10.1016/j.snb.2014.12.077.
304. Ippolito SJ, Kandasamy S, Kalantar-zadeh K, Wlodarski W, Galatsis $\mathrm{K}$, Kiriakidis $\mathrm{G}$, et al. Highly sensitive layered $\mathrm{ZnO}$ / $\mathrm{LiNbO}_{3}$ SAW device with $\mathrm{InO}_{\mathrm{x}}$ selective layer for $\mathrm{NO}_{2}$ and $\mathrm{H}_{2}$ gas sensing. Sensors Actuators B Chem. 2005 Nov;111-112:20712. http://linkinghub.elsevier.com/retrieve/pii/ S0925400505006246.

305. Leskelä M, Ritala M. Atomic layer deposition chemistry: recent developments and future challenges. Angew Chemie Int Ed. 2003 Nov 24;42(45):5548-54. http://doi.wiley.com/10.1002/anie. 200301652.

306. Kim H, Lee H-B-R, Maeng W-J. Applications of atomic layer deposition to nanofabrication and emerging nanodevices. Thin Solid Films. 2009 Feb;517(8):2563-80. https://doi.org/10.1016/j. tsf.2008.09.007.

307. George SM. Atomic layer deposition: an overview. Chem Rev. 2010 Jan 13;110(1):111-31. https://pubs.acs.org/doi/10.1021/ cr900056b.

308. Johnson RW, Hultqvist A, Bent SF. A brief review of atomic layer deposition: from fundamentals to applications. Mater Today. 2014 Jun;17(5):236-46. https://linkinghub.elsevier.com/retrieve/pii/ S1369702114001436.

309. Willmott PR, Huber JR. Pulsed laser vaporization and deposition. Rev Mod Phys. 2000 Jan 1;72(1):315-28. https://link.aps.org/doi/ 10.1103/RevModPhys.72.315.

310. Schneider CW, Lippert T. Laser Ablation and Thin Film Deposition. In 2010. p. 89-112. http://link.springer.com/10. 1007/978-3-642-13281-0_5.

311. Schaaf P. Laser processing of materials. Berlin, Heidelberg: Springer Berlin Heidelberg; 2010. p. 245. (Springer Series in Materials Science; vol. 139). http://link.springer.com/10.1007/ 978-3-642-13281-0.

312. El Sabahy J, Berthier J, Ricoul F, Jousseaume V. Toward optimized SiOCH films for BTEX detection: Impact of chemical composition on toluene adsorption. Sensors Actuators, B Chem. 2018;258:628-36. https://doi.org/10.1016/j.snb.2017.11.105.

313. Öztürk S, Kösemen A, Kösemen ZA, Kılınç N, Öztürk ZZ, Penza $\mathrm{M}$. Electrochemically growth of Pd doped $\mathrm{ZnO}$ nanorods on QCM for room temperature VOC sensors. Sensors Actuators B Chem. 2016 Jan;222:280-9. https://linkinghub.elsevier.com/retrieve/pii/ S0925400515302616.

314. Yan D, Xu P, Xiang Q, Mou H, Xu J, Wen W, et al. Polydopamine nanotubes: bio-inspired synthesis, formaldehyde sensing properties and thermodynamic investigation. J Mater Chem A. 2016;4(9):3487-93. http://xlink.rsc.org/?DOI=C6TA00396F.

315. Sabri YM, Kandjani AE, Ippolito SJ, Bhargava SK. Nanosphere monolayer on a transducer for enhanced detection of gaseous heavy metal. ACS Appl Mater Interfaces. 2015 Jan 28;7(3): 1491-9. https://pubs.acs.org/doi/10.1021/am507069z.

316. Gauglitz G. Analytical evaluation of sensor measurements. Anal Bioanal Chem. 2018 Jan 10;410(1):5-13. http://link.springer.com/ 10.1007/s00216-017-0624-z.

317. Geladi P, Esbensen K. The start and early history of chemometrics: Selected interviews. Part 1. J Chemom. 1990 Sep;4(5):337-54. http://doi.wiley.com/10.1002/cem. 1180040503 .

318. Esbensen K, Geladi P. The start and early history of chemometrics: Selected interviews. Part 2. J Chemom. 1990 Nov;4(6):389-412. http://doi.wiley.com/10.1002/cem. 1180040604 .

319. Wold S. Chemometrics; what do we mean with it, and what do we want from it? Chemom Intell Lab Syst. 1995 Nov;30(1):109-15. https://linkinghub.elsevier.com/retrieve/pii/0169743995000429.

320. Brown SD, Blank TB, Sum ST, Weyer LG. Chemometrics. Anal Chem. 1994 Jun;66(12):315-59. https://pubs.acs.org/doi/abs/10. 1021/ac00084a014. 
321. Massart DL, Vandeginste B, LMC GMB, De Jong S, Lewi PJ, Smeyers-Verbeke J. Handbook of chemometrics and qualimetrics: part a. Amsterdam: Elsevier; 1997. 867 p.

322. Vandeginste BG, Massart DL, LMC B, De Jong S, Lewi PJ, Smeyers-Verbeke J, et al. Handbook of chemometrics and qualimetrics, part b. Amsterdam: Elsevier; 1988. 876 p.

323. Brereton RG. Chemometrics: data analysis for the laboratory and chemical plant. 1st ed. John Wiley \& Sons, Ltd: Chichester; 2003. $528 \mathrm{p}$.

324. Hierlemann A, Gutierrez-Osuna R. Higher-order chemical sensing. Chem Rev. 2008 Feb;108(2):563-613. https://pubs.acs.org/ doi/10.1021/cr068116m

325. Mutihac L, Mutihac R. Mining in chemometrics. Anal Chim Acta. 2008 Mar;612(1):1-18. https://linkinghub.elsevier.com/retrieve/ pii/S0003267008003450.

326. Eriksson L, Trygg J, Wold S. A chemometrics toolbox based on projections and latent variables. J Chemom. 2014 May;28(5):33246. http://doi.wiley.com/10.1002/cem.2581.

327. Zupan J. Chemical data, ways and means, past and future. J Chemom. 2015 Jan;29(1):1-8. http://doi.wiley.com/10.1002/ cem.2653.

328. Otto M. Chemometrics: statistics and computer application in analytical chemistry. 3rd ed. Wiley VCH: Weinheim, Germany; 2016. 400 p.

329. Brereton RG, Jansen J, Lopes J, Marini F, Pomerantsev A, Rodionova $\mathrm{O}$, et al. Chemometrics in analytical chemistry - part I: history, experimental design and data analysis tools. Anal Bioanal Chem. 2017 Oct 3;409(25):5891-9. http://link.springer. com/10.1007/s00216-017-0517-1.

330. Brereton RG, Jansen J, Lopes J, Marini F, Pomerantsev A, Rodionova $\mathrm{O}$, et al. Chemometrics in analytical chemistry - part II: modeling, validation, and applications. Anal Bioanal Chem. 2018 Oct 2;410(26):6691-704. http://link.springer.com/10.1007/ s00216-018-1283-4.

331. Kiralj R, Ferreira MMC. The past, present, and future of chemometrics worldwide: some etymological, linguistic, and bibliometric investigations. J Chemom. 2006 Jun;20(6-7):24772. http://doi.wiley.com/10.1002/cem.1001.

332. Ballantine DS, Rose SL, Grate JW, Wohltjen H. Correlation of surface acoustic wave device coating responses with solubility properties and chemical structure using pattern recognition. Anal Chem. 1986 Dec;58(14):3058-66. http://pubs.acs.org/doi/abs/10. 1021/ac00127a035.

333. Rose-Pehrsson SL, Grate JW, Ballantine DS, Jurs PC. Detection of hazardous vapors including mixtures using pattern recognition analysis of responses from surface acoustic wave devices. Anal Chem. 1988 Dec 15;60(24):2801-11. https://pubs.acs.org/doi/abs/ 10.1021/ac00175a032.

334. Echeverría G, Correa E, Ruiz-Altisent M, Graell J, Puy J, López L. Characterization of fuji apples from different harvest dates and storage conditions from measurements of volatiles by gas chromatography and electronic nose. J Agric Food Chem. 2004 May;52(10):3069-76. https://pubs.acs.org/doi/10.1021/ jf035271i.

335. Joo B, Huh J, Lee D. Fabrication of polymer SAW sensor array to classify chemical warfare agents. Sensors Actuators B Chem. 2007 Jan 30;121(1):47-53. https://linkinghub.elsevier.com/ retrieve/pii/S0925400506006228.

336. Si P, Mortensen J, Komolov A, Denborg J, Møller PJ. Polymer coated quartz crystal microbalance sensors for detection of volatile organic compounds in gas mixtures. Anal Chim Acta. 2007 Aug;597(2):223-30. https://linkinghub.elsevier.com/retrieve/pii/ S0003267007010720.

337. Alizadeh T, Zeynali S. Electronic nose based on the polymer coated SAW sensors array for the warfare agent simulants classification. Sensors Actuators B Chem. 2008 Jan;129(1):41223. https://linkinghub.elsevier.com/retrieve/pii/ S0925400507006600.

338. Escuderos ME, Sánchez S, Jiménez A. Virgin olive oil sensory evaluation by an artificial olfactory system, based on Quartz Crystal Microbalance (QCM) sensors. Sensors Actuators B Chem. 2010 May 18;147(1):159-64. https://linkinghub.elsevier. com/retrieve/pii/S0925400510001024.

339. Harbeck M, Șen Z, Gürol I, Gümüș G, Musluoğlu E, Ahsen V, et al. Vic-dioximes: A new class of sensitive materials for chemical gas sensing. Sensors Actuators B Chem. 2011 Aug;156(2):673-9. https://inkinghub.elsevier.com/retrieve/pii/S0925400511001286.

340. De Cesare F, Di Mattia E, Pantalei S, Zampetti E, Vinciguerra V, Canganella F, et al. Use of electronic nose technology to measure soil microbial activity through biogenic volatile organic compounds and gases release. Soil Biol Biochem. 2011;43(10): 2094-107. https://doi.org/10.1016/j.soilbio.2011.06.009.

341. Khot LR, Panigrahi S, Lin D. Development and evaluation of piezoelectric-polymer thin film sensors for low concentration detection of volatile organic compounds related to food safety applications. Sensors Actuators, B Chem. 2011;153(1):1-10. https:// doi.org/10.1016/j.snb.2010.05.043.

342. Toniolo R, Pizzariello A, Dossi N, Lorenzon S, Abollino O, Bontempelli G. Room temperature ionic liquids as useful overlayers for estimating food quality from their odor analysis by quartz crystal microbalance measurements. Anal Chem. 2013 Aug 6;85(15):7241-7. https://pubs.acs.org/doi/10.1021/ ac401151m.

343. Deng F, Chen W, Wang J, Wei Z. Fabrication of a sensor array based on quartz crystal microbalance and the application in egg shelf life evaluation. Sensors Actuators B Chem. 2018 Jul;265: 394-402. https://linkinghub.elsevier.com/retrieve/pii/ S092540051830488X.

344. Bernabei M, Pennazza G, Santonico M, Corsi C, Roscioni C, Paolesse R, et al. A preliminary study on the possibility to diagnose urinary tract cancers by an electronic nose. Sensors Actuators B Chem. 2008 Apr 14;131(1):1-4. https://linkinghub.elsevier. com/retrieve/pii/S0925400507009872.

345. Martinelli E, Santonico M, Pennazza G, Paolesse R, D’Amico A, Di Natale C. Short time gas delivery pattern improves long-term sensor reproducibility. Sensors Actuators, B Chem. 2011;156(2): 753-9. https://doi.org/10.1016/j.snb.2011.02.034.

346. Carey WP, Beebe KR, Kowalski BR. Multicomponent analysis using an array of piezoelectric crystal sensors. Anal Chem. 1987;59(11):1529-34. http://www.scopus.com/inward/record. url?eid=2-s2.0-0023363493\&partnerID $=40 \& \mathrm{md} 5=$ b58f2ae2059b5dd1dbc7390a09819065.

347. Savitzky A, Golay MJE. Smoothing and differentiation of data by simplified least squares procedures. Anal Chem. 1964 Jul;36(8): 1627-39. https://pubs.acs.org/doi/abs/10.1021/ac60214a047.

348. Strang G. Linear Algebra and Its Applications. $4^{\text {th }}$ Ed. Brooks Cole Pub Co; 2005. 544 p.

349. Anton H, Rorres C. Elementary linear algebra. $11^{\text {th }}$ Ed. Wiley; 2014. $802 \mathrm{p}$.

350. Brereton RG. Orthogonality, uncorrelatedness, and linear independence of vectors. J Chemom. 2016 Oct;30(10):564-6. http:// doi.wiley.com/10.1002/cem.2816.

351. Brereton RG. Visualizing matrices. J Chemom. 2017 Mar;31(3): e2834. http://doi.wiley.com/10.1002/cem.2834.

352. Lundstedt T, Seifert E, Abramo L, Thelin B, Nyström Å, Pettersen $\mathrm{J}$, et al. Experimental design and optimization. Chemom Intell Lab Syst. 1998 Aug;42(1-2):3-40. https://linkinghub.elsevier.com/ retrieve/pii/S0169743998000653.

353. Callao MP. Multivariate experimental design in environmental analysis. TrAC Trends Anal Chem. 2014 Nov;62:86-92. https:// linkinghub.elsevier.com/retrieve/pii/S0165993614001745. 
354. Brereton RG. Formulating an experimental design mathematically. J Chemom. 2017 Sep;31(9):e2903. http://doi.wiley.com/10. 1002/cem.2903.

355. Box GEP, Wilson KB. On the experimental attainment of optimum conditions. J R Stat Soc Ser B. 1951;13(1):1-45. http:// www.jstor.org/stable/2983966.

356. Dempster AP. An overview of multivariate data analysis. J Multivar Anal. 1971 Sep;1(3):316-46. http://linkinghub.elsevier. com/retrieve/pii/0047259X71900066.

357. Hair JFJ, Black WC, Babin BJ, Anderson RE. Multivariate data analysis. 7th ed: Pearson Prentice Hall; 2010. p. 785.

358. Jain AK, Murty MN, Flynn PJ. Data clustering: a review. ACM Comput Surv. 1999 Sep 1 [cited 2018 Jul 31];31(3):264-1. http:// dataclustering.cse.msu.edu/papers/MSU-CSE-00-16.pdf.

359. Huberty CJ. Discriminant Analysis. Rev Educ Res. 1975 Dec;45(4):543-98. http://journals.sagepub.com/doi/10.3102/ 00346543045004543.

360. Barker M, Rayens W. Partial least squares for discrimination. J Chemom. 2003 Mar 24;17(3):166-73. http://doi.wiley.com/10. 1002/cem.785.

361. Brereton RG, Lloyd GR. Partial least squares discriminant analysis: taking the magic away. J Chemom. 2014 Apr;28(4):213-25. http://doi.wiley.com/10.1002/cem.2609.

362. Wold S, Esbensen K, Geladi P. Principal component analysis. Chemom Intell Lab Syst. 1987 Aug;2(1-3):37-52. https:// linkinghub.elsevier.com/retrieve/pii/0169743987800849.

363. Jolliffe IT. Principal component analysis. second. New York, Berlin, Heidelberg, Hong Kong, London, Milan, Paris, Tokyo: Springer Verlag; 2002. $512 \mathrm{p}$.

364. Abdi H, Williams LJ. Principal component analysis. Wiley Interdiscip Rev Comput Stat. 2010 Jul;2(4):433-59. http://doi. wiley.com/10.1002/wics.101.

365. Bro R, Smilde AK. Principal component analysis. Anal Methods. 2014;6(9):2812-31. http://xlink.rsc.org/?DOI=C3AY41907J.

366. Child D. The essentials of factor analysis. 3rd ed. Bloomsbury Academic: London, Oxforg, New York, New Delhi, Sydney; 2006. $192 \mathrm{p}$.

367. Geladi P, Kowalski BR. Partial least-squares regression: a tutorial. Anal Chim Acta. 1986;185(C):1-17. https://linkinghub.elsevier. com/retrieve/pii/0003267086800289.

368. Haenlein M, Kaplan AM. A beginner's guide to partial least squares analysis. Underst Stat. 2004 Nov;3(4):283-97. http:// www.tandfonline.com/doi/abs/10.1207/s15328031us0304_4.

369. Mahalanobis PC. On the generalised distance in statistics. In: Proceedings of the National Institute of Sciences of India; 1936. p. $49-55$

370. Brereton RG. The Mahalanobis distance and its relationship to principal component scores. J Chemom. 2015 Mar;29(3):143-5. http://doi.wiley.com/10.1002/cem.2692.

371. Brereton RG, Lloyd GR. Re-evaluating the role of the Mahalanobis distance measure. J Chemom. 2016 Apr;30(4): 134-43. http://doi.wiley.com/10.1002/cem.2779.

372. Barkó G, Abonyi J, Hlavay J. Application of fuzzy clustering and piezoelectric chemical sensor array for investigation on organic compounds. Anal Chim Acta. 1999 Oct;398(2-3):219-26. https://linkinghub.elsevier.com/retrieve/pii/S0003267099003773.

373. Hotelling H. Analysis of a complex of statistical variables into principal components. J Educ Psychol. 1933;24(6):417-41. http://content.apa.org/journals/edu/24/6/417.

374. Wold H. Nonlinear estimation by Iterative Least Squares Procedures. In: David FN, Neyman J, editors. Research papers in statistics, Festschrift for J Neyman. London, New York: Wiley; 1966.

375. Gorsuch RL. Factor analysis. Boca Raton: Taylor \& Francis; 1983. $464 \mathrm{p}$.
376. Mumyakmaz B, Özmen A, Ebeoğlu MA, Taşaltın C, Gürol İ. A study on the development of a compensation method for humidity effect in QCM sensor responses. Sensors Actuators B Chem. 2010 May 18;147(1):277-82. https://linkinghub.elsevier.com/retrieve/ pii/S0925400510002315.

377. Yong AG, Pearce S. A beginner's guide to factor analysis: focusing on exploratory factor analysis. Tutor Quant Methods Psychol. 2013 Oct 1;9(2):79-94. http://www.tqmp.org/RegularArticles/ vol09-2/p079.

378. Fisher RA. The use of multiple measurements in taxonomic problems. Ann Eugen. 1936 Sep;7(2):179-88. http://doi.wiley.com/ 10.1111/j.1469-1809.1936.tb02137.x.

379. Wold S. Pattern recognition by means of disjoint principal components models. Pattern Recognit. 1976 Jul;8(3):127-39. https:// linkinghub.elsevier.com/retrieve/pii/0031320376900145.

380. Wold S, Sjöström M. SIMCA: A method for analyzing chemical data in terms of similarity and analogy. In 1977. p. 243-82. https:// pubs.acs.org/doi/abs/10.1021/bk-1977-0052.ch012.

381. Jain AK. Data clustering: 50 years beyond K-means. Pattern Recognit Lett. 2010 Jun;31(8):651-66. https://doi.org/10.1016/j. patrec.2009.09.011.

382. Wold S, Sjöström M, Eriksson L. PLS-regression: A basic tool of chemometrics. Chemom Intell Lab Syst. 2001 Oct;58(2):109-30. https://linkinghub.elsevier.com/retrieve/pii/S0169743901001551.

383. Garthwaite PH. An interpretation of partial least squares. J Am Stat Assoc. 1994 Mar;89(425):122-7. http://www.tandfonline. com/doi/abs/10.1080/01621459.1994.10476452.

384. Schölkopf B, Smola A, Müller K-R. Kernel principal component analysis. In: Gerstner W, Germond A, Hasler M, Nicoud J-D, editors. Artificial neural networks - ICANN'97. Berlin, Heidelberg: Springer Berlin Heidelberg; 1997. p. 583-8.

385. Schölkopf B, Smola A, Müller K-R. Nonlinear component analysis as a kernel eigenvalue problem. Neural Comput [Internet]. 1998 Jul;10(5):1299-319 Available from:. http://www. mitpressjournals.org/doi/10.1162/089976698300017467.

386. Brereton RG, Lloyd GR. Partial least squares discriminant analysis for chemometrics and metabolomics: How scores, loadings, and weights differ according to two common algorithms. J Chemom. 2018 Apr;32(4):e3028. http://doi.wiley.com/10.1002/ cem.3028.

387. McCarthy J, Minsky ML, Rochester N, Shannon CE. A proposal for the dartmouth summer research project on artificial intelligence, August 31, 1955. AI Mag. 2006 Dec 15;27(4 SEArticles). https://aaai.org/ojs/index.php/aimagazine/article/view/ 1904.

388. James CD, Aimone JB, Miner NE, Vineyard CM, Rothganger FH, Carlson KD, et al. A historical survey of algorithms and hardware architectures for neural-inspired and neuromorphic computing applications. Biol Inspired Cogn Archit. 2017 Jan;19:49-64. https:// linkinghub.elsevier.com/retrieve/pii/S2212683X16300561.

389. Isenhour TL, Jurs PC. Some chemical applications of machine intelligence. Anal Chem. 1971 Aug 29;43(10):20A-35A. https:// pubs.acs.org/doi/abs/10.1021/ac60304a715.

390. Hopke PK. The evolution of chemometrics. Anal Chim Acta. 2003 Dec;500(1-2):365-77. https://linkinghub.elsevier.com/ retrieve/pii/S0003267003009449.

391. Jansson PA. Neural networks: an overview. Anal Chem. 1991 Mar 15;63(6):357A-62A. https://pubs.acs.org/doi/abs/10.1021/ ac00006a739.

392. Zupan J, Gasteiger J. Neural networks: A new method for solving chemical problems or just a passing phase? Anal Chim Acta. 1991 Jul;248(1):1-30. https://linkinghub.elsevier.com/retrieve/pii/ S000326700080865X.

393. Smits JRM, Melssen WJ, Buydens LMC, Kateman G. Using artificial neural networks for solving chemical problems. Part I. Multi-layer feed-forward networks. Chemom Intell Lab Syst. 
1994 Feb;22(2):165-89. https://linkinghub.elsevier.com/retrieve/ pii/0169743993E00353.

394. Melssen WJ, Smits JRM, Buydens LMC, Kateman G. Using artificial neural networks for solving chemical problems. Chemom Intell Lab Syst. 1994 May;23(2):267-91. https://linkinghub. elsevier.com/retrieve/pii/0169743993E00364.

395. Despagne F, Luc MD. Neural networks in multivariate calibration. Analyst. 1998;123(11):157-78. http://xlink.rsc.org/?DOI= a805562i.

396. Haykin S. Neural networks. a comprehensive fundation. 2nd ed: Prentice Hall International, Inc.; 1999. p. 842.

397. Hinton GE. Reducing the dimensionality of data with neural networks. Science (80- ). 2006 Jul 28;313(5786):504-7. http://www. sciencemag.org/cgi/doi/10.1126/science.1127647.

398. Vapnik VN. Estimation of dependences based on empirical data (in Russian; English translation: Springer Verlag, New York, 1982). 1st ed. Moskow: Nauka; 1979.

399. Burges CJC. A tutorial on support vector machines for pattern recognition. Data Min Knowl Discov. 1998;2(2):121-67. https:// doi.org/10.1023/A:1009715923555.

400. Belousov AI, Verzakov SA, von Frese J. Applicational aspects of support vector machines. J Chemom. 2002 Aug;16(8-10):482-9. http://doi.wiley.com/10.1002/cem.744.

401. Thissen U, van Brakel R, de Weijer A, Melssen W, Buydens LM. Using support vector machines for time series prediction. Chemom Intell Lab Syst. 2003 Nov;69(1-2):35-49. https:// linkinghub.elsevier.com/retrieve/pii/S0169743903001114.

402. Brereton RG, Lloyd GR. Support Vector Machines for classification and regression. Analyst. 2010;135(2):230-67. http://xlink.rsc. org/?DOI=B918972F.

403. Luts J, Ojeda F, Van de Plas R, De Moor B, Van Huffel S, Suykens JAK. A tutorial on support vector machine-based methods for classification problems in chemometrics. Anal Chim Acta. 2010 Apr;665(2):129-45. https://linkinghub. elsevier.com/retrieve/pii/S0003267010003132.

404. Brereton RG. Pattern recognition in chemometrics. Chemom Intell Lab Syst. 2015 Dec;149:90-6. https://doi.org/10.1016/j. chemolab.2015.06.012.

405. Holland JH. Genetic algorithms. Sci Am. 1992;267(1):66-73. http://www.jstor.org/stable/24939139.

406. Leardi R. Genetic algorithms in chemometrics and chemistry: a review. J Chemom. 2001 Aug;15(7):559-69. http://doi.wiley. com/10.1002/cem.651.

407. van Leeuwen JA, Buydens LMC, Vandeginste BGM, Kateman G. Expert systems in chemical analysis. TrAC Trends Anal Chem. $1990 \mathrm{Feb} ; 9(2): 49-54$. https://linkinghub.elsevier.com/retrieve/pii/ 0165993690850253 .

408. Gerritsen M, van Leeuwen JA, Vandeginste BGM, Buydens L, Kateman G. Expert systems for multivariate calibration, trendsetters for the wide-spread use of chemometrics. Chemom Intell Lab Syst. 1992 Aug;15(2-3):171-84. https://linkinghub.elsevier.com/ retrieve/pii/016974399285007P.

409. Rius FX. Expert systems in trace analysis. Anal Chim Acta. 1993 Nov;283(1):518-27. https://linkinghub.elsevier.com/retrieve/pii/ 000326709385265L

410. Chang SM, Iwasaki Y, Suzuki M, Tamiya E, Karube I, Muramatsu H. Detection of odorants using an array of piezoelectric crystals and neural-network pattern recognition. Anal Chim Acta. 1991;249(2):323-9. http://www.scopus.com/inward/ record.url?eid=2-s2.0-0025914317\&partnerID $=40 \& \mathrm{md} 5=$ 1cc70bd1263ba26efa627978cf33d274.

411. Marco S, Pardo A, Davide FAM, Di Natale C, D'Amico A, Hierlemann A, et al. Different strategies for the identification of gas sensing systems. Sensors Actuators B Chem. 1996;34(1-3): 213-23. http://www.sciencedirect.com/science/article/pii/ S0925400597800019.
412. McAlernon P, Slater JM, Lau K-T. Mapping of chemical functionality using an array of quartz crystal microbalances in conjunction with Kohonen self-organizing maps. Analyst. 1999;124(6): 851-7. http://xlink.rsc.org/?DOI=a900422j.

413. Saraoğlu HM, Edin B. E-nose system for anesthetic dose level detection using artificial neural network. J Med Syst. 2007 Oct 15;31(6):475-82. http://link.springer.com/10.1007/s10916-0079087-7.

414. Muckley ES, Lynch J, Kumar R, Sumpter B, Ivanov IN. Sensors and actuators B : Chemical PEDOT : PSS / QCM-based multimodal humidity and pressure sensor. Sensors Actuators B Chem. 2016;236:91-8. https://doi.org/10.1016/j.snb.2016.05.054.

415. McCulloch WS, Pitts W. A logical calculus of the ideas immanent in nervous activity. Bull Math Biophys. 1943 Dec;5(4):115-33. http://link.springer.com/10.1007/BF02478259.

416. Pitts W, McCulloch WS. How we know universals the perception of auditory and visual forms. Bull Math Biophys. 1947 Sep;9(3): 127-47. http://link.springer.com/10.1007/BF02478291.

417. Hebb DO. The organization of behavior. New York: John Wiley \& Sons, Inc.; 1949. 365 p.

418. Squire L, Berg D, Bloom F, du Lac S, Ghosh A, Spitzer N. Fundamental neuroscience. $3^{\text {rd }}$ Ed. Squire L, editor. Amsterdam, Boston, Heidelberg, London, New York, Oxford, Paris, San Diego, San Francisco, Singapore, Sydney, Tokyo: Academic Press is an imprint of Elsevier; 2008. 1277 p.

419. Amerman E. Human anatomy \& physiology. 2nd ed: Pearson; 2018. p. 1248.

420. Rosenblatt F. The perceptron: A probabilistic model for information storage and organization in the brain. Psychol Rev. 1958;65(6):386-408. http://doi.apa.org/getdoi.cfm?doi=10.1037/ h0042519.

421. Minsky M, Papert SA. Perceptrons: an introduction to computational geometry. Cambridge, Mass: MIT Press; 1969. p. 258.

422. Hopfield JJ. Neural networks and physical systems with emergent collective computational abilities. Proc Natl Acad Sci. 1982 Apr 1;79(8):2554-8. http://www.pnas.org/cgi/doi/10.1073/pnas.79.8. 2554.

423. Rumelhart DE, Hinton GE, Williams RJ. Learning representations by back-propagating errors. Nature. 1986 Oct;323(6088):533-6. http://www.nature.com/articles/323533a0.

424. Rumelhart DE, McClelland JL, PDP RG. Parallel distributed processing, volume 1. explorations in the microstructure of cognition: foundations: MIT Press; 1986. p. 567.

425. McClelland JL, Rumelhart DE, PDP RG. Parallel distributed processing, volume 2. explorations in the microstructure of cognition: psychological and biological models: MIT Press; 1986. p. 632.

426. Wythoff BJ. Backpropagation neural networks. Chemom Intell Lab Syst. 1993 Feb;18(2):115-55. https://linkinghub.elsevier. com/retrieve/pii/016974399380052J.

427. Mendel JM, McLaren RW. Reinforcement-learning control and pattern recognition systems. Math Sci Eng. 1970;66(C):287318. https://doi.org/10.1016/S0076-5392(08)60497-X.

428. Schmidhuber J. Deep learning in neural networks: An overview. Neural Networks. 2015 Jan;61:85-117. https://linkinghub. elsevier.com/retrieve/pii/S0893608014002135.

429. Boser BE, Guyon IM, Vapnik VN. A training algorithm for optimal margin classifiers. In: Proceedings of the fifth annual workshop on Computational learning theory - COLT '92. New York, New York, USA: ACM Press; 1992. p. 144-52. http://portal.acm. org/citation.cfm?doid=130385.130401.

430. Mammone A, Turchi M, Cristianini N. Support vector machines. Wiley Interdiscip Rev Comput Stat. 2009 Nov;1(3):283-9. https:// onlinelibrary.wiley.com/doi/abs/10.1002/wics.49.

431. Brereton RG. Points, vectors, linear independence and some introductory linear algebra. J Chemom. 2016 Jul;30(7):358-60. http://doi.wiley.com/10.1002/cem.2802. 
432. Cortes C, Vapnik V. Support-vector networks. Mach Learn. 1995 Sep;20(3):273-97. http://link.springer.com/10.1007/ BF00994018.

433. Pearce TC, Schiffman SS, Nagle HT, Gardner JW, editors. Handbook of machine olfaction. Weinheim, FRG: Wiley-VCH Verlag GmbH \& Co. KGaA; 2002. 592 p. http://doi.wiley.com/ $10.1002 / 3527601597$.

434. Davide FAM, Di Natale C, D'Amico A, Hierlemann A, Mitrovics J, Schweizer M, et al. Structure identification of non-linear models for QMB polymer-coated sensors. Sensors Actuators B Chem. 1995 Apr;25(1-3):830-42. https://linkinghub.elsevier.com/ retrieve/pii/0925400595851852.

435. Davide FAM, Di Natale C, D'Amico A, Hierlemann A, Mitrovics J, Schweizer M, et al. Dynamic calibration of QMB polymer-coated sensors by Wiener kernel estimation. Sensors Actuators B Chem. 1995 Jun;27(1-3):275-85. https://linkinghub.elsevier. com/retrieve/pii/092540059401601D.

436. Sharma P, Ghosh A, Tudu B, Bhuyan LP, Tamuly P, Bhattacharyya N, et al. Detection of linalool in black tea using a quartz crystal microbalance sensor. Sensors Actuators B Chem. 2014 Jan;190:318-25. https://doi.org/10.1016/j.snb.2013.08.088.

437. Khanniche S, Mathieu D, Pereira F, Frenois C, Colin D, Barthet C, et al. Quantitative evaluation of the responses of a gravimetric gas sensor based on mesoporous functionalized silica: Application to 2,4-DNT and TNT detection. Sensors Actuators, B Chem. 2017;248:470-80. https://doi.org/10.1016/j.snb.2017.03.137.

438. Nimsuk N, Nakamoto T. Improvement of capability for classifying odors in dynamically changing concentration using QCM sensor array and short-time Fourier transform. Sensors Actuators B Chem. 2007 Nov 15;127(2):491-6. https://linkinghub.elsevier. com/retrieve/pii/S0925400507003267.

439. Iglesias L, Boudjiet MT, Dufour I. Discrimination and concentration measurement of different binary gas mixtures with a simple resonator through viscosity and mass density measurements. Sensors Actuators, B Chem. 2019;285(January):487-94. https:// doi.org/10.1016/j.snb.2019.01.070.

440. Duffus J. Worth H, editors. Fundamental toxicology for chemists: The Royal Society of Chemistry; 1996. 2184 p. https://doi.org/10. 1039/9781847550941.

441. Crowl DA. Understanding Explosions. $1^{\text {st }}$ Ed. Wiley-AIChE; 2003. $214 \mathrm{p}$

442. Abraham MH, Sánchez-Moreno R, Gil-Lostes J, Acree WE, Enrique Cometto-Muñiz J, Cain WS. The biological and toxicological activity of gases and vapors. Toxicol Vitr. $2010 \mathrm{Mar} ; 24(2)$ : 357-62. https://linkinghub.elsevier.com/retrieve/pii/ S0887233309003385.

443. Dahl A. Dose concepts for inhaled vapors and gases. Toxicol Appl Pharmacol. 1990 Apr;103(2):185-97. https://linkinghub.elsevier. com/retrieve/pii/0041008X9090221F.

444. Hayes AW, Kruger CL. Hayes' principles and methods of toxicology: CRC Press; 2014. https://www.taylorfrancis.com/books/ 9781842145371.

445. Gupta PK. Toxicologic hazards of solvents, gases, vapors, and other chemicals BT - concepts and applications in veterinary toxicology: an interactive guide. In: Gupta PK, editor. Cham: Springer International Publishing; 2019. p. 121-42. https://doi. org/10.1007/978-3-030-22250-5_6.

446. Kuempel ED, Sweeney LM, Morris JB, Jarabek AM. Advances in inhalation dosimetry models and methods for occupational risk assessment and exposure limit derivation. J Occup Environ Hyg. 2015 Nov 25;12(sup1):S18-40. http://www.tandfonline.com/doi/ full/10.1080/15459624.2015.1060328.

447. Nelms MD, Simmons JE, Edwards SW. Adverse outcome pathways to support the assessment of chemical mixtures. In: Chemical mixtures and combined chemical and nonchemical stressors. Cham: Springer International Publishing; 2018. p.
177-201. http://link.springer.com/10.1007/978-3-319-56234-6 7.

448. Hecker M, LaLone CA. Adverse outcome pathways: moving from a scientific concept to an internationally accepted framework. Environ Toxicol Chem. 2019 Jun 27;38(6):1152-63. https:// onlinelibrary.wiley.com/doi/abs/10.1002/etc. 4385 .

449. Patlewicz G. perspectives on the development, evaluation and application of in silico approaches for predicting toxicity. 2019; https://epa.figshare.com/articles/Perspectives_on_the Development_Evaluation_and_Application_of_in_Silico_ Approaches_for_Predicting_Toxicity/10299371.

450. Globally Harmonized System of Classification and Labelling of Chemicals (GHS). UN; 2019. (Globally Harmonized System of Classification and Labelling of Chemicals (GHS)). https://www. un-ilibrary.org/environment-and-climate-change/globallyharmonized-system-of-classification-and-labelling-of-chemicalsghs_f8fbb7cb-en.

451. Winder C, Azzi R, Wagner D. The development of the globally harmonized system (GHS) of classification and labelling of hazardous chemicals. J Hazard Mater. 2005 Oct;125(1-3):29-44. https://linkinghub.elsevier.com/retrieve/pii/ S030438940500292X.

452. U.S. Department of Labor. A Guide toThe Globally Harmonized System of Classification and Labeling of Chemicals (GHS). Osha. 2006;90. https://web.archive.org/web/20070702005153/http:// www.osha.gov/dsg/hazcom/ghs.html\#3.0.

453. IFA DEGUV. GESTIS International Limit Values. 2020 [cited 2020 Jan 20]. https://limitvalue.ifa.dguv.de/.

454. IFA. GESTIS. [cited 2020 Jan 20]. http://gestis.itrust.de/nxt/ gateway.dll/gestis en/000000.xml?f=templates $\& \mathrm{fn}=$ default. htm\&vid=gestiseng:sdbeng.

455. Lukey BJ, Romano JA Jr, Salem H, editors. Chemical warfare agents: biomedical and psychological effects, medical countermeasures, and emergency response: CRC Press; 2019. 826 p.

456. Picard B, Chataigner I, Maddaluno J, Legros J. Introduction to chemical warfare agents, relevant simulants and modern neutralisation methods. Org Biomol Chem. 2019;17(27):6528 37. https://doi.org/10.1039/c9ob00802k.

457. Pitschmann V. Overall view of chemical and biochemical weapons. Toxins (Basel). 2014 Jun 4;6(6):1761-84. http://www. mdpi.com/2072-6651/6/6/1761.

458. Lavoie J, Srinivasan S, Nagarajan R. Using cheminformatics to find simulants for chemical warfare agents. J Hazard Mater. 2011 Oct;194:85-91. https://linkinghub.elsevier.com/retrieve/pii/ S0304389411009587.

459. Zabetakis MG. Flammability characteristics of combustible gases and vapors. Bull Bur Mines. 1965;627:121.

460. Lewis B, von Elbe G. Combustion, flames and explosions of gases. $3^{\text {rd }}$ Ed. Academic Press / Elsevier; 1987. 731 p.

461. Glassman I, Yetter RA. Combustion. 4th ed: Academic Press / Elsevier; 2008. p. 800.

462. McAllister S, Chen J-Y, Fernandez-Pello AC. Fundamentals of combustion processes. New York, NY: Springer; 2011. p. 328. (Mechanical Engineering Series). http://link.springer.com/10. 1007/978-1-4419-7943-8.

463. Carson P, Mumford C. Hazardous chemicals handbook. 2nd ed: Butterworth-Heinemann; 2002. p. 608.

464. Grate JW, Patrash SJ, Abraham MH, Du CM. Selective vapor sorption by polymers and cavitands on acoustic wave sensors: is this molecular recognition? Anal Chem. 1996 Jan;68(5):913-7. https://pubs.acs.org/doi/10.1021/ac950518z.

465. Grate JW, Patrash SJ, Kaganove SN, Wise BM. Hydrogen bond acidic polymers for surface acoustic wave vapor sensors and arrays. Anal Chem. 1999 Mar;71(5):1033-40. https://pubs.acs.org/ doi/10.1021/ac9810011. 
466. Hierlemann A, Zellers ET, Ricco AJ. Use of linear solvation energy relationships for modeling responses from polymer-coated acoustic-wave vapor sensors. Anal Chem. $2001 \mathrm{Jul} ; 73(14)$ : 3458-66. http://pubs.acs.org/doi/abs/10.1021/ac010083h.

467. Cotton AF, Wilkinson G, Murillo CA, Bochmann M. Advanced inorganic chemistry. 6th ed: Wiley-Interscience; 1999. p. 1376.

468. Atkins P, Overton T, Rourke J, Weller M, Armstrong F. Shriver and atkins' inorganic chemistry. 5th ed: Oxford University Press; 2009. p. 864.

469. Atkins P, de Paula J. Atkins' physical chemistry. 10th ed: Oxford University Press; 2014. p. 1040.

470. Smith MB, March J. March's advanced organic chemistry. WileyInterscience; 2007. $2384 \mathrm{p}$.

471. McMurry J. Fundamentals of organic chemistry. 9th ed: Brooks Cole Pub Co; 2015. p. 1512.

472. Poling BE, Praushitz JM, O'Connell JP. The properties of gases and liquids. 5th ed: McGraw-Hill Professional; 2000. p. 768.

473. Yaws CL. The yaws handbook of vapor pressure. 2nd ed: Elsevier Inc; 2015. p. 336. https://linkinghub.elsevier.com/retrieve/pii/ C20140035903.

474. Rumble J. CRC handbook of chemistry and physics. 100th ed; 2019. p. 1700.

475. Dortmund Data Bank. 2020. http://www.ddbst.com/.

476. El Sabahy J, Berthier J, Bonnet L, Matheron M, Bordy T, Yeromonahos $\mathrm{C}$, et al. Toluene-organic thin films partition coefficients analyzed with Langmuir adsorption theory and finite elements simulations. Sensors Actuators B Chem. 2014 Oct;202: 941-8. https://doi.org/10.1016/j.snb.2014.05.041.

477. Zhang K, Fan G, Hu R, Li G. Enhanced dibutyl phthalate sensing performance of a quartz crystal microbalance coated with Au-decorated $\mathrm{ZnO}$ porous microspheres. Sensors. 2015 Aug 27;15(9): 21153-68. http://www.mdpi.com/1424-8220/15/9/21153.

478. Yang M, He J. Graphene oxide as quartz crystal microbalance sensing layers for detection of formaldehyde. Sensors Actuators, B Chem. 2016;228:486-90. https://doi.org/10.1016/j.snb.2016. 01.046 .

479. Wang L, Gao J, Xu J. QCM formaldehyde sensing materials: Design and sensing mechanism. Sensors Actuators B Chem. 2019 Aug;293(April):71-82. https://linkinghub.elsevier.com/ retrieve/pii/S0925400519305738.

480. Cui S, Yang L, Wang J, Wang X. Fabrication of a sensitive gas sensor based on $\mathrm{PPy} / \mathrm{TiO}_{2}$ nanocomposites films by layer-by-layer self-assembly and its application in food storage. Sensors Actuators, B Chem. 2016;233:337-46. https://doi.org/10.1016/j. snb.2016.04.093.

481. Chen W, Deng F, Xu M, Wang J, Wei Z, Wang Y. GO/Cu $\mathrm{Cu}_{2} \mathrm{O}$ nanocomposite based QCM gas sensor for trimethylamine detection under low concentrations. Sensors Actuators, B Chem. 2018 Nov;273(June):498-504. https://linkinghub.elsevier.com/retrieve/ pii/S0925400518311675.

482. Wang W, Hu H, Liu X, He S, Pan Y, Zhang C, et al. Development of a room temperature saw methane gas sensor incorporating a supramolecular cryptophane a coating. Sensors. 2016 Jan 7;16(1):73. http://www.mdpi.com/1424-8220/16/1/73.

483. Shen B, Yang P, Liu X-L, Zhang H, Cao S. Fabrication and characterizations of SAW methane sensor based on cryptophane-E membrane. J Ambient Intell Humaniz Comput. 2018 Aug 27: 0123456789. http://link.springer.com/10.1007/s12652-018-09821.

484. Yang M, He J. Tailoring the structure of metal oxide nanostructures towards enhanced sensing properties for environmental applications. J Colloid Interface Sci. 2012;368(1):41-8. https://doi. org/10.1016/j.jcis.2011.09.061.

485. Di Pietrantonio F, Benetti M, Cannatà D, Verona E, Palla-Papavlu A, Dinca V, et al. Volatile toxic compound detection by surface acoustic wave sensor array coated with chemoselective polymers deposited by laser induced forward transfer: Application to sarin. Sensors Actuators, B Chem. 2012;174:158-67. https://doi.org/10. 1016/j.snb.2012.07.106.

486. Sayago I, Matatagui D, Fernández MJ, Fontecha JL, Jurewicz I, Garriga R, et al. Graphene oxide as sensitive layer in Love-wave surface acoustic wave sensors for the detection of chemical warfare agent simulants. Talanta. 2016;148:393-400. https://doi.org/ 10.1016/j.talanta.2015.10.069.

487. Chen D, Zhang K, Zhou H, Fan G, Wang Y, Li G, et al. A wireless-electrodeless quartz crystal microbalance with dissipation DMMP sensor. Sensors Actuators B Chem. 2018 May;261:408 17. https://doi.org/10.1016/j.snb.2018.01.105.

488. Eslami MR, Alizadeh N. Ultrasensitive and selective QCM sensor for detection of trace amounts of nitroexplosive vapors in ambient air based on polypyrrole-Bromophenol blue nanostructure. Sensors Actuators B Chem. 2019 Jan;278(September 2018):5563. https://linkinghub.elsevier.com/retrieve/pii/ S0925400518316800.

489. Yang M, He J, Hu X, Yan C, Cheng Z, Zhao Y, et al. Copper oxide nanoparticle sensors for hydrogen cyanide detection: Unprecedented selectivity and sensitivity. Sensors Actuators, B Chem. 2011;155(2):692-8. https://doi.org/10.1016/j.snb.2011. 01.031 .

490. Yang M, He J, Hu M, Hu X, Yan C, Cheng Z. Synthesis of copper oxide nanoparticles and their sensing property to hydrogen cyanide under varied humidity conditions. Sensors Actuators, B Chem. 2015;213:59-64. https://doi.org/10.1016/j.snb.2015.02. 055.

491. Prantl E, Kohl B, Ryvlin D, Biegger P, Wadepohl H, Rominger F, et al. Microporous triptycene-based affinity materials on quartz crystal microbalances for tracing of illicit compounds. Chempluschem. 2019 Sep 7;84(9):1239-44. https://onlinelibrary. wiley.com/doi/abs/10.1002/cplu.201900189.

492. Sadek AZ, Wlodarski W, Shin K, Kaner RB, Kalantar-zadeh K. A layered surface acoustic wave gas sensor based on a polyaniline/ $\mathrm{In}_{2} \mathrm{O}_{3}$ nanofibre composite. Nanotechnology. $2006 \mathrm{Sep}$ 14;17(17):4488-92. http://stacks.iop.org/0957-4484/17/i=17/a= 034?key=crossref.450351f8ae9c234a3e0954bc1b991740.

493. Bayram A, Özbek C, Senel M, Okur S. CO gas sorption properties of ferrocene branched chitosan derivatives. Sensors Actuators B Chem. 2017 Mar;241:308-13. https://linkinghub.elsevier.com/ retrieve/pii/S0925400516314083.

494. Tian Y, Qu K, Zeng X. Investigation into the ring-substituted polyanilines and their application for the detection and adsorption of sulfur dioxide. Sensors Actuators B Chem. 2017 Oct;249:42330.https://linkinghub.elsevier.com/retrieve/pii/ S0925400517306585.

495. Lv Y, Xu P, Yu H, Xu J, Li X. Ni-MOF-74 as sensing material for resonant-gravimetric detection of ppb -level CO. Sensors Actuators B Chem. 2018 Jun;262:562-9. http://linkinghub. elsevier.com/retrieve/pii/S0925400518303344.

496. Li D, Zu X, Ao D, Tang Q, Fu Y, Guo Y, et al. High humidity enhanced surface acoustic wave ( $\mathrm{SAW}$ ) $\mathrm{H}_{2} \mathrm{~S}$ sensors based on solgel $\mathrm{CuO}$ films. Sensors Actuators B Chem. 2019 Sep;294(April): 55-61. https://linkinghub.elsevier.com/retrieve/pii/ S0925400519305337.

497. Viespe C, Miu D. Surface acoustic wave sensor with $\mathrm{Pd} / \mathrm{ZnO}$ bilayer structure for room temperature hydrogen detection. Sensors. 2017 Jun 29;17(7):1529. http://www.mdpi.com/1424$8220 / 17 / 7 / 1529$

498. McKeown SJ, Wang X, Yu X, Goddard LL. Realization of palladium-based optomechanical cantilever hydrogen sensor. Microsystems Nanoeng. 2017;3(October 2016):1-6. https://doi. org/10.1038/micronano.2016.87.

499. Farahani H, Wagiran R, Hamidon M. Humidity sensors principle, mechanism, and fabrication technologies: a comprehensive 
review. Sensors. 2014 Apr 30;14(5):7881-939. http://www.mdpi. com/1424-8220/14/5/7881.

500. Xuan W, He M, Meng N, He X, Wang W, Chen J, et al. Fast response and high sensitivity $\mathrm{ZnO} /$ glass surface acoustic wave humidity sensors using graphene oxide sensing layer. Sci Rep. 2015 May 26;4(1):7206. http://www.nature.com/articles/ srep07206.

501. Zhu Y, Chen J, Li H, Zhu Y, Xu J. Synthesis of mesoporous $\mathrm{SnO}_{2}-\mathrm{SiO}_{2}$ composites and their application as quartz crystal microbalance humidity sensor. Sensors Actuators B Chem. 2014 Mar;193:320-5. http://linkinghub.elsevier.com/retrieve/pii/ S092540051301441X

502. Yoo HY, Bruckenstein S. A novel quartz crystal microbalance gas sensor based on porous film coatings. A high sensitivity porous poly(methylmethacrylate) water vapor sensor. Anal Chim Acta. 2013;785:98-103. https://doi.org/10.1016/j.aca.2013.04.052.

503. Sahm M, Oprea A, Bârsan N, Weimar U. Interdependence of ammonia and water sorption in polyacrylic acid layers. Sensors Actuators, B Chem. 2008 Mar 14;130(1):502-7. http:// linkinghub.elsevier.com/retrieve/pii/S0925400507007460.

504. Tsukahara Y, Hirayama O, Takeda N, Oizumi T, Fukushi H, Sato $\mathrm{N}$, et al. A novel method and an equipment for generating the standard moisture in gas flowing through a pipe. Sensors. 2018 Oct 13;18(10):3438. http://www.mdpi.com/1424-8220/18/10/ 3438.

505. Sharma P, Ghosh A, Tudu B, Sabhapondit S, Baruah BD, Tamuly $\mathrm{P}$, et al. Monitoring the fermentation process of black tea using QCM sensor based electronic nose. Sensors Actuators, B Chem. 2015;219:146-57. https://doi.org/10.1016/j.snb.2015.05.013.

506. Ali SB, Ghatak B, Gupta SD, Debabhuti N, Chakraborty P, Sharma $\mathrm{P}$, et al. Detection of 3-Carene in mango using a quartz crystal microbalance sensor. Sensors Actuators, B Chem. 2016;230:791-800. https://doi.org/10.1016/j.snb.2016.03.005.

507. Barié N, Bücking M, Stahl U, Rapp M. Detection of coffee flavour ageing by solid-phase microextraction/surface acoustic wave sensor array technique (SPME/SAW). Food Chem. 2015 Jun;176: 212-8. https://linkinghub.elsevier.com/retrieve/pii/ S0308814614019438.

508. Kabir KMM, Sabri YM, Myers L, Harrison I, Boom E, Coyle VE, et al. Investigating the cross-interference effects of alumina refinery process gas species on a SAW based mercury vapor sensor. Hydrometallurgy. 2017;170:51-7. https://doi.org/10.1016/j. hydromet.2016.05.015.

509. Kabir KMM, Sabri YM, Lay B, Ippolito SJ, Bhargava SK. A silver electrode based surface acoustic wave (SAW) mercury vapor sensor: a physio-chemical and analytical investigation. RSC Adv. 2016;6(43):36362-72. http://xlink.rsc.org/?DOI= C6RA03148J.

510. Sabri YM, Ippolito SJ, O'Mullane AP, Tardio J, Bansal V, Bhargava SK. Creating gold nanoprisms directly on quartz crystal microbalance electrodes for mercury vapor sensing. Nanotechnology. 2011 Jul 29;22(30):305501. http://stacks.iop. org $/ 0957-4484 / 22 / \mathrm{i}=30 / \mathrm{a}=305501$ ? key=crossref. 6036f10cde4c6013196818e1617238da.

511. Di Natale C, Buchholt K, Martinelli E, Paolesse R, Pomarico G, D'Amico A, et al. Investigation of quartz microbalance and ChemFET transduction of molecular recognition events in a metalloporphyrin film. Sensors Actuators B Chem. 2009 Jan;135(2):560-7. https://linkinghub.elsevier.com/retrieve/pii/ S0925400508006710.

512. Li C-L, Lu C-J. Establishing linear solvation energy relationships between VOCs and monolayer-protected gold nanoclusters using quartz crystal microbalance. Talanta. 2009 Aug;79(3):851-5. https://linkinghub.elsevier.com/retrieve/pii/S0039914009003865.

513. Chevallier E, Scorsone E, Girard HA, Pichot V, Spitzer D, Bergonzo P. Metalloporphyrin-functionalised diamond nano- particles as sensitive layer for nitroaromatic vapours detection at room-temperature. Sensors Actuators, B Chem. 2010;151(1): 191-7. https://doi.org/10.1016/j.snb.2010.09.022.

514. Latif U, Rohrer A, Lieberzeit PA, Dickert FL. QCM gas phase detection with ceramic materials-VOCs and oil vapors. Anal Bioanal Chem. 2011 Jun 8;400(8):2457-62. http://link.springer. com/10.1007/s00216-011-4684-1.

515. Ding B, Wang X, Yu J, Wang M. Polyamide 6 composite nanofiber/net functionalized by polyethyleneimine on quartz crystal microbalance for highly sensitive formaldehyde sensors. J Mater Chem. 2011;21(34):12784. http://xlink.rsc.org/?DOI= cljm11847a.

516. $\mathrm{Xu} \mathrm{P}, \mathrm{Yu} \mathrm{H}, \mathrm{Li} \mathrm{X}$. Functionalized mesoporous silica for microgravimetric sensing of trace chemical vapors. Anal Chem. 2011 May;83(9):3448-54. https://pubs.acs.org/doi/10.1021/ ac200015c.

517. Jakubik W, Krzywiecki M, Maciak E, Urbańczyk M. Bi-layer nanostructures of $\mathrm{CuPc}$ and $\mathrm{Pd}$ for resistance-type and SAW-type hydrogen gas sensors. Sensors Actuators B Chem. 2012 Dec;175: 255-62. https://linkinghub.elsevier.com/retrieve/pii/ S0925400512010805.

518. Yan Y, Lu D, Zhou H, Hou H, Zhang T, Wu L, et al. Polyanilinemodified quartz crystal microbalance sensor for detection of formic acid gas. Water, Air, Soil Pollut. 2012 Mar 28;223(3): 1275-80. http://link.springer.com/10.1007/s11270-011-0943-1.

519. Wang X, Wang W, Li H, Fu C, Ke Y, He S. Development of a $\mathrm{SnO}_{2} / \mathrm{CuO}$-coated surface acoustic wave-based $\mathrm{H}_{2} \mathrm{~S}$ sensor with switch-like response and recovery. Sensors Actuators, B Chem. 2012;169:10-6. https://doi.org/10.1016/j.snb.2012.01.002.

520. Minh VA, Tuan LA, Huy TQ, Hung VN, Van Quy N. Enhanced $\mathrm{NH}_{3}$ gas sensing properties of a QCM sensor by increasing the length of vertically orientated $\mathrm{ZnO}$ nanorods. Appl Surf Sci. 2013;265:458-64. https://doi.org/10.1016/j.apsusc.2012.11.028.

521. Di Pietrantonio F, Cannatà D, Benetti M, Verona E, Varriale A, Staiano M, et al. Detection of odorant molecules via surface acoustic wave biosensor array based on odorant-binding proteins. Biosens Bioelectron. 2013 Mar;41(1):328-34. https://inkinghub. elsevier.com/retrieve/pii/S0956566312005702.

522. Wang Y, Ding P, Hu R, Zhang J, Ma X, Luo Z, et al. A dibutyl phthalate sensor based on a nanofiber polyaniline coated quartz crystal monitor. Sensors. 2013 Mar 18;13(3):3765-75. http:// www.mdpi.com/1424-8220/13/3/3765.

523. Boutamine M, Bellel A, Sahli S, Segui Y, Raynaud P. Hexamethyldisiloxane thin films as sensitive coating for quartz crystal microbalance based volatile organic compounds sensors. Thin Solid Films. 2014;552:196-203. https://doi.org/10.1016/j. tsf.2013.12.016.

524. Ayad MM, Salahuddin N, Minisy IM. Detection of some volatile organic compounds with chitosan-coated quartz crystal microbalance. Des Monomers Polym. 2014;17(8):795-802. https://doi.org/ 10.1080/15685551.2014.918019.

525. Strle D, Štefane B, Zupanič E, Trifkovič M, Maček M, Jakša G, et al. Sensitivity comparison of vapor trace detection of explosives based on chemo-mechanical sensing with optical detection and capacitive sensing with electronic detection. Sensors. 2014 Jun 27;14(7):11467-91. http:/www.mdpi.com/1424-8220/14/7/ 11467.

526. Tang YL, Li ZJ, Ma JY, Guo YJ, Fu YQ, Zu XT. Ammonia gas sensors based on $\mathrm{ZnO} / \mathrm{SiO}_{2}$ bi-layer nanofilms on ST-cut quartz surface acoustic wave devices. Sensors Actuators, B Chem. 2014;201:114-21. https://doi.org/10.1016/j.snb.2014.04.046.

527. Kabir KMM, Sabri YM, Matthews GI, Jones LA, Ippolito SJ, Bhargava SK. Selective detection of elemental mercury vapor using a surface acoustic wave (SAW) sensor. Analyst. 2015;140(16):5508-17. http://xlink.rsc.org/?DOI= C5AN00360A. 
528. Matsuguchi M, Takaoka K, Kai H. $\mathrm{HCl}$ gas adsorption/desorption properties of poly(N-isopropylacrylamide) brushes grafted onto quartz resonator for gas-sensing applications. Sensors Actuators B Chem. 2015 Mar;208:106-11. http://linkinghub.elsevier.com/ retrieve/pii/S0925400514013495.

529. Benz M, Benz L, Patel SV. High temperature mass detection using a carbon nanotube bilayer modified quartz crystal microbalance as a GC detector. Anal Chem. 2015 Mar 3;87(5):2779-87. https:// pubs.acs.org/doi/10.1021/ac504101a.

530. Hu M, He J, Yang M, Hu X, Yan C, Cheng Z. Hydrothermal synthesis of nanostructured flower-like $\mathrm{Ni}(\mathrm{OH})_{2}$ particles and their excellent sensing performance towards low concentration HCN gas. RSC Adv. 2015;5(34):26823-31. https://doi.org/10. 1039/C5RA02742J.

531. Luo W, Deng J, Fu Q, Zhou D, Hu Y, Gong S, et al. Nanocrystalline $\mathrm{SnO}_{2}$ film prepared by the aqueous sol-gel method and its application as sensing films of the resistance and SAW $\mathrm{H}_{2} \mathrm{~S}$ sensor. Sensors Actuators, B Chem. 2015;217:119-28. https://doi.org/10.1016/j.snb.2014.10.078.

532. Wang S-H, Shen C-Y, Su J-M, Chang S-W. A room temperature nitric oxide gas sensor based on a copper-ion-doped polyaniline/ tungsten oxide nanocomposite. Sensors. 2015 Mar 24;15(4): 7084-95. http://www.mdpi.com/1424-8220/15/4/7084.

533. Xu S, Li C, Li H, Li M, Qu C, Yang B. Carbon dioxide sensors based on a surface acoustic wave device with a graphene-nickelL-alanine multilayer film. J Mater Chem C. 2015;3(16):3882-90. http://xlink.rsc.org/?DOI=C4TC02986K.

534. Zhang H-D, Yan X, Zhang Z-H, Yu G-F, Han W-P, Zhang J-C, et al. Electrospun PEDOT:PSS/PVP Nanofibers for CO gas sensing with quartz crystal microbalance technique. Int J Polym Sci. 2016;2016:1-6. http://www.hindawi.com/journals/ijps/2016/ $3021353 /$

535. Kumar A, Brunet J, Varenne C, Ndiaye A, Pauly A. Phthalocyanines based QCM sensors for aromatic hydrocarbons monitoring: Role of metal atoms and substituents on response to toluene. Sensors Actuators, B Chem. 2016;230:320-9. https:// doi.org/10.1016/j.snb.2016.02.032

536. Clément P, Del Castillo PE, Gonzalez O, Calavia R, Lucat C, Llobet E, et al. Gas discrimination using screen-printed piezoelectric cantilevers coated with carbon nanotubes. Sensors Actuators, B Chem. 2016;237:1056-65. https://doi.org/10.1016/j.snb.2016. 07.163 .

537. Jia Y, Yu H, Zhang Y, Dong F, Li Z. Cellulose acetate nanofibers coated layer-by-layer with polyethylenimine and graphene oxide on a quartz crystal microbalance for use as a highly sensitive ammonia sensor. Colloids Surfaces B Biointerfaces. 2016 Dec;148:263-9. https://linkinghub.elsevier.com/retrieve/pii/ S092777651630649X.

538. Şen Z, Tarakci DK, Gürol I, Ahsen V, Harbeck M. Governing the sorption and sensing properties of titanium phthalocyanines by means of axial ligands. Sensors Actuators B Chem. 2016 Jun;229:581-6. https://linkinghub.elsevier.com/retrieve/pii/ S0925400516301459.

539. Yang L, Yin C, Zhang Z, Zhou J, Xu H. The investigation of hydrogen gas sensing properties of SAW gas sensor based on palladium surface modified $\mathrm{SnO}_{2}$ thin film. Mater Sci Semicond Process. 2017 Mar;60(July 2016):16-28. https://doi.org/10.1016/ j.mssp.2016.11.042.

540. Öztürk S, Kösemen A, Șen Z, Kılınç N, Harbeck M. Poly(3methylthiophene) thin films deposited electrochemically on qcms for the sensing of volatile organic compounds. Sensors. $2016 \mathrm{Mar}$ 23;16(4):423. http://www.mdpi.com/1424-8220/16/4/423.

541. Marcu A, Viespe C. Surface acoustic wave sensors for hydrogen and deuterium detection. Sensors. 2017 Jun 16;17(6):1417. http:// www.mdpi.com/1424-8220/17/6/1417.
542. Rana L, Gupta R, Tomar M, Gupta V. ZnO/ST-Quartz SAW resonator: An efficient $\mathrm{NO}_{2}$ gas sensor. Sensors Actuators, B Chem. 2017;252(2):840-5. https://doi.org/10.1016/j.snb.2017. 06.075 .

543. Tokura Y, Nakada G, Moriyama Y, Oaki Y, Imai H, Shiratori S. Ultrasensitive detection of methylmercaptan gas using layered manganese oxide nanosheets with a quartz crystal microbalance sensor. Anal Chem. 2017 Nov 21;89(22):12123-30. http://pubs. acs.org/doi/10.1021/acs.analchem.7b02738.

544. Wang L, Zhu Y, Xiang Q, Cheng Z, Chen Y, Xu J. One novel humidity-resistance formaldehyde molecular probe based hydrophobic diphenyl sulfone urea dry-gel: Synthesis, sensing performance and mechanism. Sensors Actuators B Chem. 2017 Nov;251:590-600. https://doi.org/10.1016/j.snb.2017.05.074.

545. Ali SB, Ghatak B, Debabhuti N, Sharma P, Ghosh A, Tudu B, et al. Detection of $\beta$-caryophyllene in mango using a quartz crystal microbalance sensor. Sensors Actuators B Chem. 2018 Feb;255: 3064-73. https://linkinghub.elsevier.com/retrieve/pii/ S0925400517317975.

546. Triyana K, Sembiring A, Rianjanu A, Hidayat S, Riowirawan R, Julian T, et al. Chitosan-based quartz crystal microbalance for alcohol sensing. Electronics. 2018 Sep 8;7(9):181. http://www. mdpi.com/2079-9292/7/9/181.

547. Tang Y, Ao D, Li W, Zu X, Li S, Fu YQ. $\mathrm{NH}_{3}$ sensing property and mechanisms of quartz surface acoustic wave sensors deposited with $\mathrm{SiO}_{2}, \mathrm{TiO}_{2}$, and $\mathrm{SiO}_{2}-\mathrm{TiO}_{2}$ composite films. Sensors Actuators B Chem. 2018 Jan;254:1165-73. https://linkinghub. elsevier.com/retrieve/pii/S0925400517314090.

548. Stahl U, Voigt A, Dirschka M, Barié N, Richter C, Waldbaur A, et al. Long-term capability of polymer-coated surface transverse wave sensors for distinguishing vapors of similar hydrocarbons. Sensors Actuators, B Chem. 2018;274(August):560-4. https://doi. org/10.1016/j.snb.2018.08.013.

549. Fragoso-Mora JR, Matatagui D, Bahos FA, Fontecha J, Fernandez MJ, Santos JP, et al. Gas sensors based on elasticity changes of nanoparticle layers. Sensors Actuators, B Chem. 2018;268:93-9. https://doi.org/10.1016/j.snb.2018.04.045.

550. Miu D, Birjega R, Viespe C. Surface acoustic wave hydrogen sensors based on nanostructured $\mathrm{Pd} / \mathrm{WO}_{3}$ bilayers. Sensors. 2018 Oct 26;18(11):3636. http://www.mdpi.com/1424-8220/18/ 11/3636.

551. Rianjanu A, Roto R, Julian T, Hidayat S, Kusumaatmaja A, Suyono E, et al. Polyacrylonitrile nanofiber-based quartz crystal microbalance for sensitive detection of safrole. Sensors. $2018 \mathrm{Apr}$ 10;18(4):1150. http://www.mdpi.com/1424-8220/18/4/1150.

552. Diltemiz SE, Ecevit K. High-performance formaldehyde adsorption on $\mathrm{CuO} / \mathrm{ZnO}$ composite nanofiber coated QCM sensors. J Alloys Compd. 2019 Apr;783:608-16. https://linkinghub. elsevier.com/retrieve/pii/S0925838818347935.

553. Li M, Kan H, Chen S, Feng X, Li H, Li C, et al. Colloidal quantum dot-based surface acoustic wave sensors for $\mathrm{NO}_{2}$-sensing behavior. Sensors Actuators, B Chem. 2019;287(2):241-9. https://doi. org/10.1016/j.snb.2019.02.042.

554. Gurusamy JT, Putrino G, Jeffery RD, Silva KKMBD, Martyniuk M, Keating A, et al. MEMS based hydrogen sensing with partsper-billion resolution. Sensors Actuators B Chem. 2019 Feb;281(July 2018):335-42. https://linkinghub.elsevier.com/ retrieve/pii/S0925400518313662.

555. Shu L, Wang X, Yan D, Fan L, Wu W. the investigation of hightemperature saw oxygen sensor based on $\mathrm{ZnO}$ films. Materials (Basel). 2019 Apr 15;12(8):1235. https://www.mdpi.com/19961944/12/8/1235.

556. Venkatasubramanian A, Sauer VTK, Westwood-Bachman JN, Cui K, Xia M, Wishart DS, et al. Porous nanophotonic optomechanical beams for enhanced mass adsorption. ACS 
Sensors. 2019 May 24;4(5):1197-202. https://pubs.acs.org/doi/ 10.1021/acssensors.8b01366.

557. Wang W, Liu X, Mei S, Jia Y, Liu M, Xue X, et al. Development of a $\mathrm{Pd} / \mathrm{Cu}$ nanowires coated SAW hydrogen gas sensor with fast response and recovery. Sensors Actuators, B Chem.
2019;287(January):157-64. https://doi.org/10.1016/j.snb.2019. 02.047 .

Publisher's note Springer Nature remains neutral with regard to jurisdictional claims in published maps and institutional affiliations. 